\title{
URBAN FLOOD ANALYSIS IN OKLAHOMA CITY, OKLAHOMA
}

By Robert L. Tortorelli, Thomas L. Huntzinger, DeRoy L. Bergman, and Alphonse L. Patneaude, Jr.

U.S. GEOLOGICAL SURVEY OPEN-FILE REPORT 83-26

Prepared in cooperation with the CITY OF OKLAHOMA CITY, OKLAHOMA

Oklahoma City, Oklahoma 


\title{
UNITED STATES DEPARTMENT OF THE INTERIOR
} JAMES G. WATT, Secretary

\author{
GEOLOGICAL SURVEY \\ Dallas L. Peck, Director
}

For additional information writo to:

James H. Irwin, District Chief

U.S. Geological Survey

Water Resources Division

Rm. 621, Old Post Office Bldg.

215 Dean A. Mc Gee Avenue

Oklahoma City, Ok 73102

Telephone : $405-231-4256$
Copies of this report

can be purchased from:

Open-File Services Section

Western Distribution Branch

U.S. Geological Survey

Box 25425, Federal Center

Lakewood, Colorado 80225

(Telephone: (303) 234-5888) 
Paqe

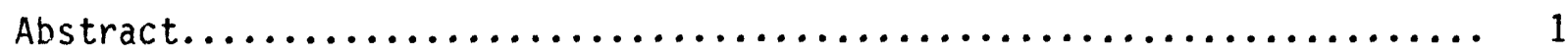

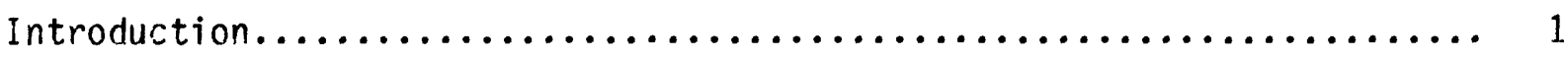

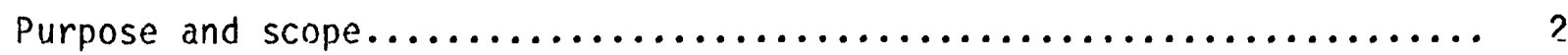

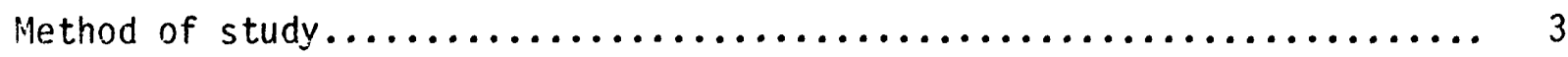

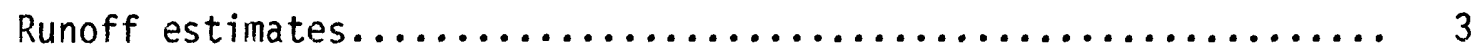

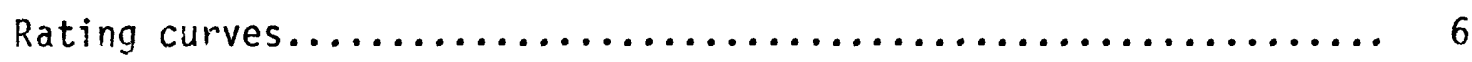

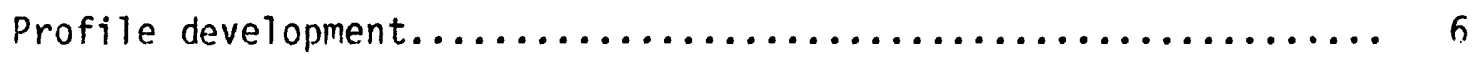

Limitations................................. 7

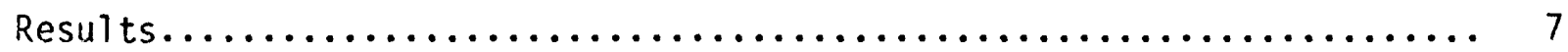

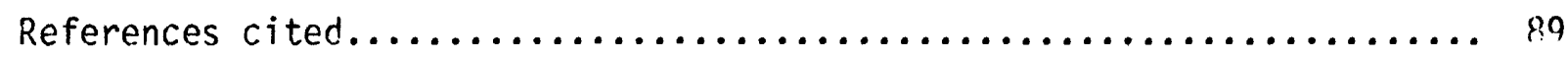

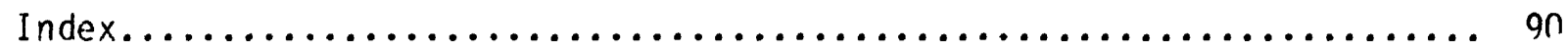

\section{ILLUSTRATIONS}

Figure 1. The relationship of the urban adjustment factor, $R_{L}$, to the percentage of area impervious and served by storm sewer. 
Table 1. Page number of Table 2 and FIS Map Panel numbers for flooding sources of Oklahoma City, Oklahoma in downstream order by basin..................................

Table 2. Flood data for Oklahoma City, Oklahoma, flood sources under existing conditions and with urban factor, $R_{L}=4.1 \ldots \ldots \ldots$.

\section{CONVERSION FACTORS}

Inch-pound units used in this report may he converted to International System of Units (SI) by the following conversion factors:

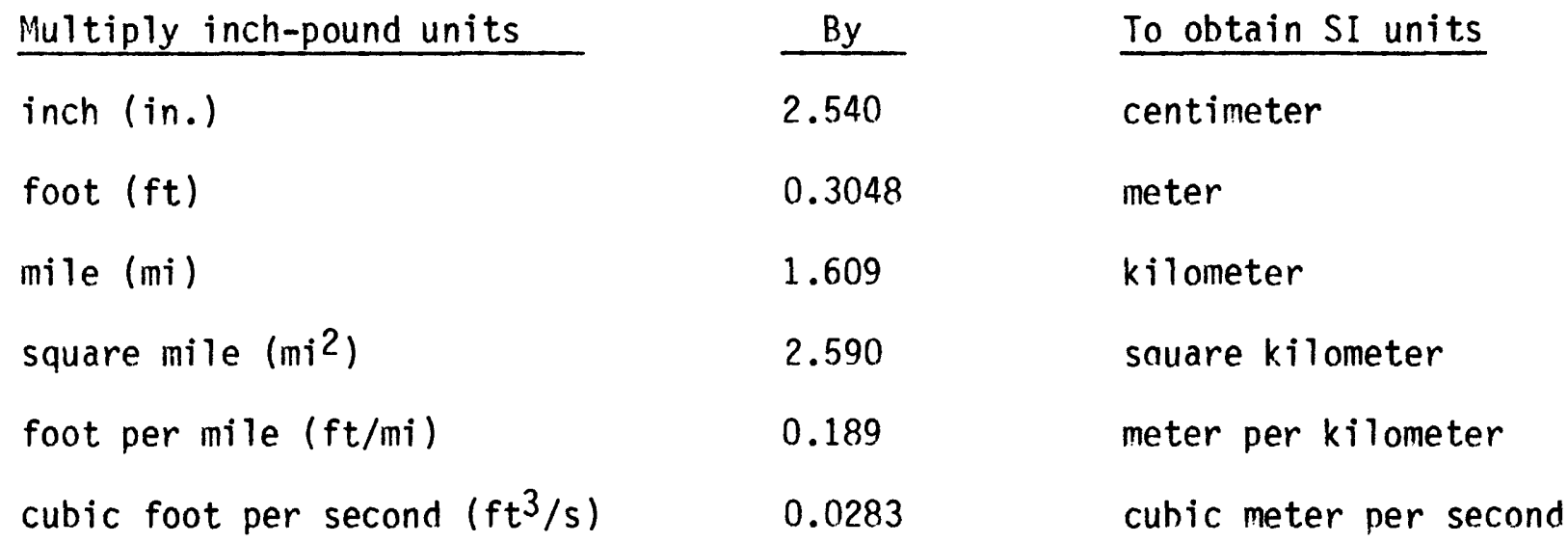




\author{
URBAN FLOOD ANALYSIS IN OKLAHOMA CITY, OKLAHOMA \\ By \\ Robert L. Tortorelli, Thomas L. Huntzinger, \\ DeRoy L. Bergman, and Alphonse L. Patneaude, Jr.
}

ABSTRACT

Flood insurance study information from the Federal Emergency Management Agency is utilized to estimate future flood hazard in Oklahoma City, Oklahoma. Techniques are described for estimating future urban runoff estimates. A method of developing stream cross section rating curves is explained. Future runoff estimates are used in conjuction with the rating curves to develop an estimate of 50- and 100- year flood profiles that would result from future urban development.

\title{
INTRODUCTION
}

Rapid urban development of drainage basins in Oklahoma City, OKlahoma, has increased the need for information on flood hazard potential in the area. The U.S. Geological Survey, under contract by the Federal Emergency Management Agency (FEMA), has developed flood information for 435 miles of stream channel in the Oklahoma City metropolitan area (FEMA, 1982). Current rates of development in the drainage basins within OKlahoma City are so rapid that analysis based on present conditions becomes outdated in a short time. In an attempt to keep results of flood analysis current, the existing flood insurance study information was re-analyzed based on an estimated future development the City considers to be reasonable. 


\section{PURPOSE AND SCOPE}

The results of this study will provide the City of Oklahoma City, Oklahoma, with flood hazard information based on analysis which assumes urban development as planned in the future. The stream reaches included in the analysis are those streams in the City of $0 \mathrm{k}$ lahoma City, Oklahoma, Flood Insurance Study (FEMA, 1982). The North Canadian River and the Canadian River main channels are excluded. Five major drainage basins are included in the study:

North Canadian Piver basin,

Canadian River basin,

Deep Fork basin,

Deer Creek basin,

Hog Creek basin,

Flood hazard information that was developed in this study include:

1. Stage-discharge relations for cross sections (flood plain shape) at selected locations along the study reaches based on flood insurance study results,

2. Estimates of flood discharge based on planned development $\left(R_{L}=4.1\right)$ for the 1 and 2 percent probability floods (100- and 50 - year recurrence intervals), and

3. Flood profiles based on planned development $\left(R_{L}=4.1\right)$ for the 1 and 2 percent probability floods (100- and 50- year recurrence intervals). 
METHOD OF STUDY

The techniques of hydrology and hydraulics used in this study are summarized by Huntzinger (1978). Basin characteristics were used to compute expected flood discharges for future conditions. Channel stage-discharge relations for each cross section were developed from four plotted stage-discharge points in the Flood Insurance Study. The future flood profiles were then determined by finding the elevations corresponding to the future flood discharge at each cross section.

\section{Runoff estimates}

Flood discharges at any given point on a stream depend on the physical and climatological characteristics of the area drained by the stream. Those characteristics that are the most significant in determining flood discharges in rural 0klahoma streams are drainage area, main-channel slope, and mean annual precipitation. Equations developed by Thomas and Corley (1977) relate the peak discharge of floods in rural basins having recurrence intervals of $2-, 5-, 10-, 25-, 50-, 100-$, and 500-years to these stream basin characteristics.

Urban-basin peak flood discharges are normally larger than rural-basin peak flood discharges for any given frequency. Any increases in peak discharge generally depend on the amount and type of urban development in the ba$\sin$.

Although urban runoff information for OKlahoma City is limited, Sauer (1974) has developed an approach for estimating flood discharge for urban basins in Oklahoma. The technique is based upon the assumption that the urban 
flood frequency curve can be estimated by interpolating between the rural flood frequency curve (lower limiting discharges) and the flood frequency curve for a completely developed basin (upper limiting discharges). This interpolation for intermediate stages of development is hased on an urban adjustment factor, $R_{L}$, defined by Leopold (1968) as the ratio of the urban 2-year peak discharge to the rural 2-year peak discharge. Figure 1 illustrates the relationship between the urban adjustment factor, $R_{L}$, and the percentage of the basin which is impervious and area served by storm sewers adapted from Leopold (1968) by Sauer (1974).

The rural or undeveloped flood frequency curve $\left(R_{L}=1\right)$ can be estimated from Thomas and Corley (1977) as noted above. The completely developed flood frequency curve $\left(R_{L}=7\right)$ is approximated by the rainfall intensity frequency curve assuming 100 percent runoff. Interpolation of $Q_{N U}$, the urban peak discharge for frequency $N$, can be determined for the general equation (Sauer, 1974 ),

$$
\frac{Q_{N U}}{Q_{2}}=\frac{7 R_{X}\left(R_{L}-1\right)}{6}+\frac{\left(7-R_{L}\right)}{6} \frac{Q_{N}}{Q_{2}}
$$

where $Q_{N}$ is the peak discharge for rural conditions for frequency $N$; $R X$ is the ratio of the 2-year, 24-hour rainfall to the N-year, 24-hour rainfall; and the other terms are as previously defined. Sauer (1974) provides information on the assumptions made and the equations used in developing the methor.

The City of OkTahoma City has used an $R_{L}$ of 4.1 as representative of ful1 urban development on the basins in this study. Therefore, estimated future urban discharges used in this report were computed using an $R_{L}$ of 4.1 in the above general equation for $\mathrm{N}=50$ and 100 years. 


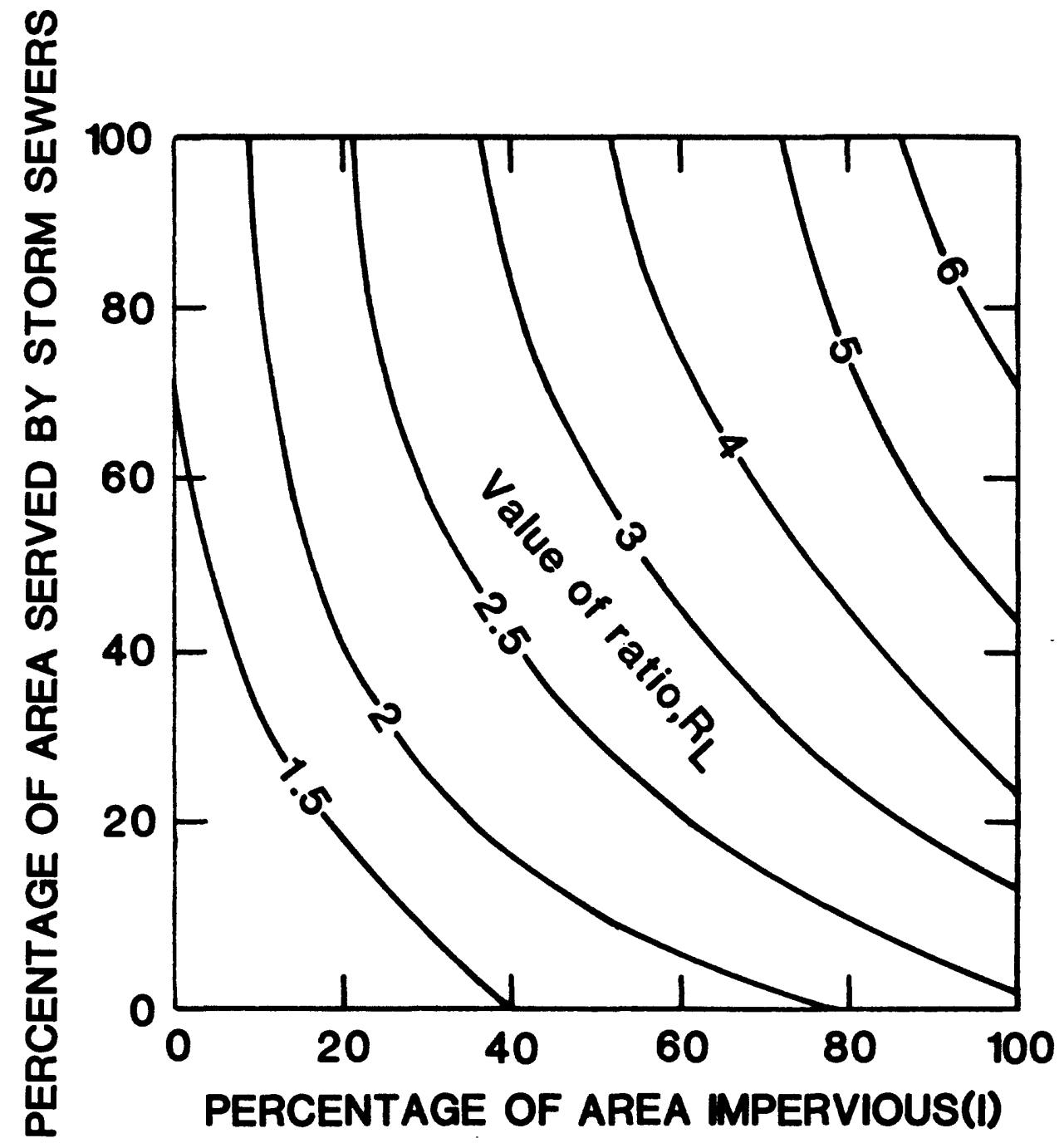

Figure 1.--The relationship of the urban adjustment. factor, $R_{L}$, to the percentage of the area impervious and served hy storm sewer. (Adapted from Leopold, 1968, by Sauer, 1974). 


\section{Rating curves}

A cross-section rating curve, or stage-discharge relation, is determined by plotting the discharge against the water-surface elevation (stage) corresponding to that discharge for a range of discharges. Flood discharges were determined under present conditions $(1980)$ for the 10-, 50-, 100-, and 500year floods for all stream reaches included in the Oklahoma City Flood Insurance Study. Discharges were computed at points along stream channels where significant drainage area changes took place such as at the confluence of major tributaries. Water-surface elevations at each cross section for the four flood discharges were computed using a step-backwater computer progam to route the selected discharges upstream.

The four stage-discharge points for selected cross sections in the Oklahoma City Flood Insurance Study were plotted on logarithmic plotting paper. The plots were graphically extended as needed to cover the range of future urban discharges used in the study.

\section{Profile development}

The cross-section rating curves and estimated future urban flood discharge relations may be used to define flood profiles for any urban development plan. To define these profiles, urban discharges of each desired frequency are computed for the planned urbanized basin above each cross section. Next, the elevation corresponding to each discharge is determined from the rating curve at each cross section. The elevations are then plotted against cross section location along the channel to obtain flood profiles for the planned urbanization. 
Water-surface elevations for the 50- and 100-year flood discharges for an $R_{L}=4.1$ at selected cross sections in OKlahoma City were determined in this manner.

\section{Limitations}

Techniques described in this report are designed to make optimum use of information previously generated from a flood insurance study. In using this information for an urban study, approximations must be made where available data do not exactly meet the user's requirements; therefore, the application of these techniques have limitations.

Any alteration of the hydraulic characteristics of the stream channel such as a change in shape, slope, or vegetative cover may change the shape of the rating curves. Most streams in Oklahoma City have mild slopes that result in a subcritical state of flow. Subcritical flows at a cross section are downstream dependent. Therefore, channel changes at a particular point along a stream will not affect the rating curve downstream but may cause changes in rating curves immediately upstream; in fact, all rating curves upstream may be changed. The techniques described in this report assume there will be no change in the hydraulic characteristics of the stream channel with urban development.

\section{RESULTS}

The results of this study are presented in table 2 .

Table 1 is included to assist in determining the stream cross section locations on appropriate Flood Insurance Study Map Panels and as a 
cross-reference in finding the applicable flood data on Table 2.

Table 2 lists the flood data for both existing conditions and full urban development in downstream basin order. First, flooding source showing the stream cross section designation and location are given. Next, the discharge and water-surface elevation for the 10-, 50-, 100-, and 500-year floods are tabulated for existing conditions from the OKlahoma City Flood Insurance Study.Then, the discharge and water-surface elevation data for the 50-, and 100-year floods for an urban factor, $R_{L}$, of 4.1 is presented.

Note elevations and discharges based on existing conditions on upper Bluff Creek and Crooked Oak Creek were computed by the Corps of Engineers in which a 90 percent urbanization was assumed. Therefore, the flood elevations listed under urban factor $R_{L}=4.1$ are sometimes lower than the existing conditions from the flood insurance study because of the difference in computation methods and urbanization assumptions. 


\begin{tabular}{|c|c|c|c|c|c|c|c|c|c|c|c|c|c|}
\hline 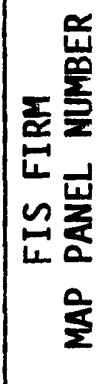 & & $\stackrel{5}{\infty}$ & $\stackrel{n}{\circ}$ & 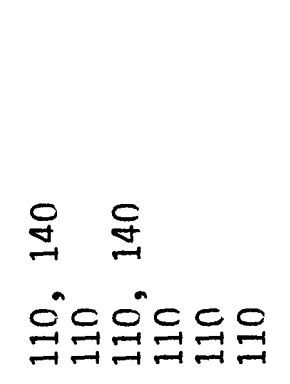 & $\stackrel{g}{\exists}$ & 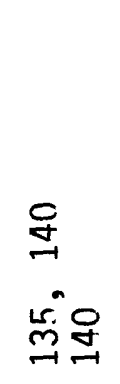 & 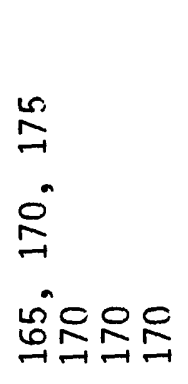 & 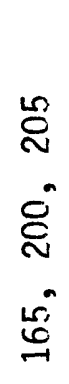 & $\begin{array}{l}0 \\
\\
0 \\
0 \\
0\end{array}$ & 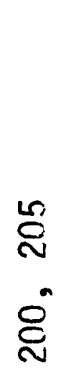 & $\begin{array}{l}\stackrel{\Sigma}{E} \\
\stackrel{\Sigma}{E}\end{array}$ & $\begin{array}{l}\text { L } \\
\text { E } \\
\text { ¿ }\end{array}$ & 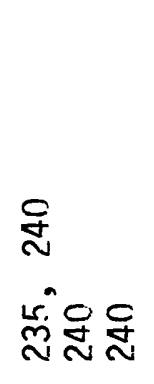 \\
\hline 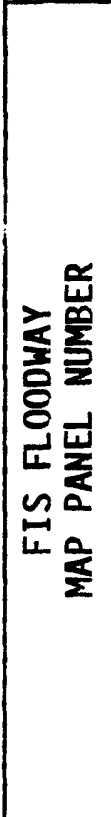 & & $\begin{array}{l}0 \\
5 \\
\infty \\
0 \\
2\end{array}$ & $\begin{array}{l}\nwarrow \\
\infty \\
\circ \\
\text { ! }\end{array}$ & 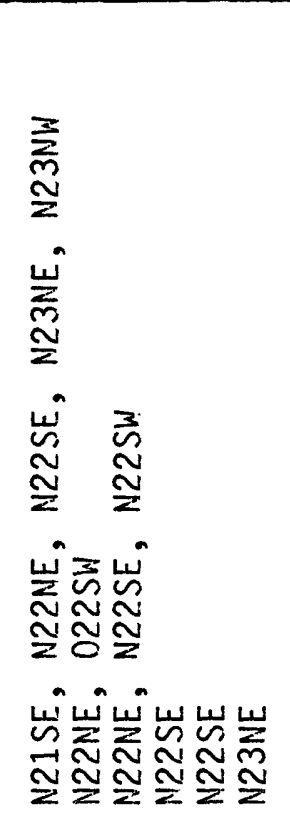 & 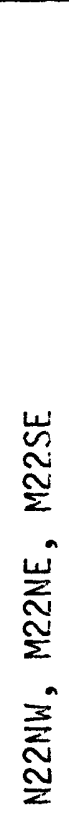 & 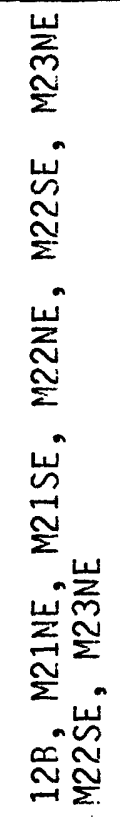 & 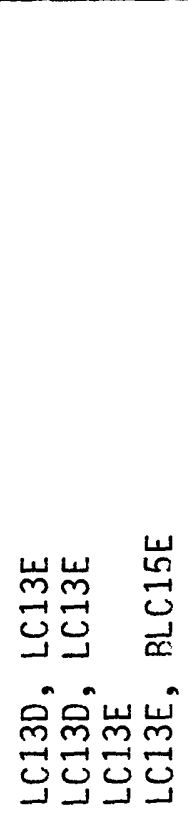 & 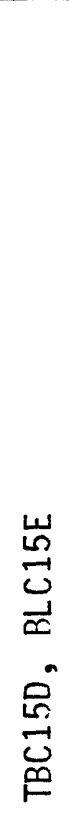 & 占 & $\stackrel{\Xi}{\Xi}$ & $\underset{\sim}{\sim}$ & $\stackrel{\sim}{\beth}$ & 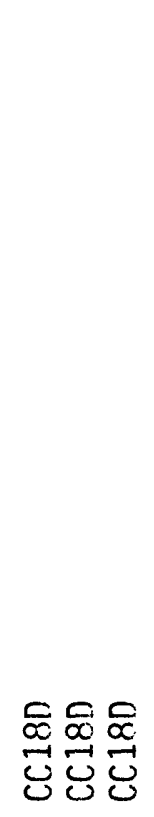 \\
\hline 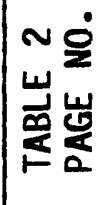 & & $=$ & $\exists$ & 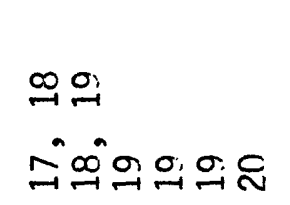 & ㅇ & $\begin{array}{l}\underset{N}{N} \\
\grave{N} \approx\end{array}$ & 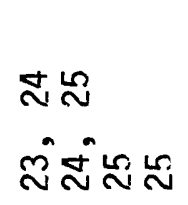 & $\stackrel{心}{\stackrel{N}{N}}$ & $\stackrel{\approx}{\approx}$ & $\Sigma$ & $\bar{N}$ & $\stackrel{\infty}{\sim}$ & న \\
\hline 岩 & 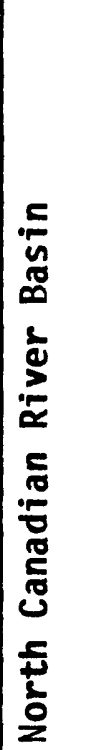 & 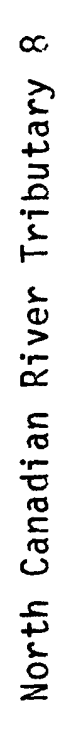 & 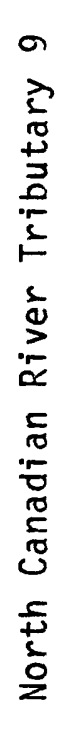 & 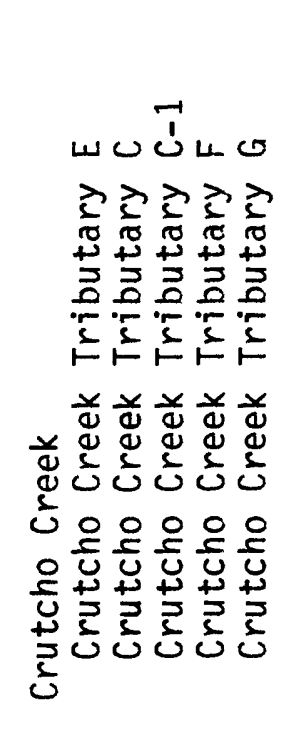 & 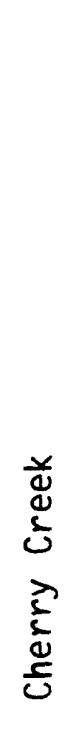 & 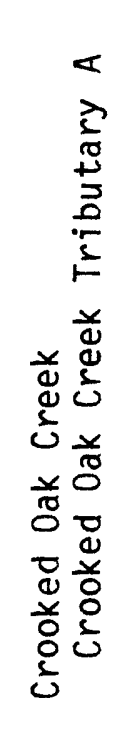 & 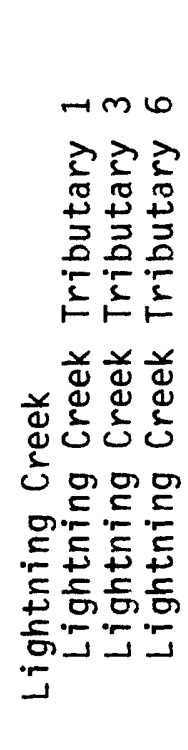 & 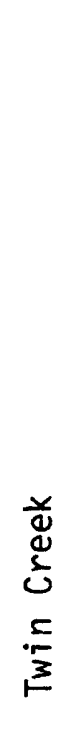 & 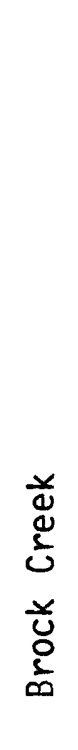 & 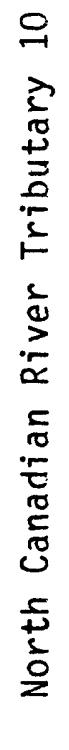 & 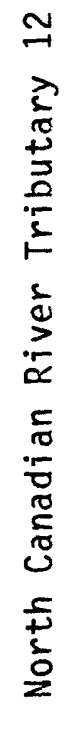 & 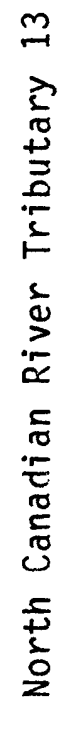 & 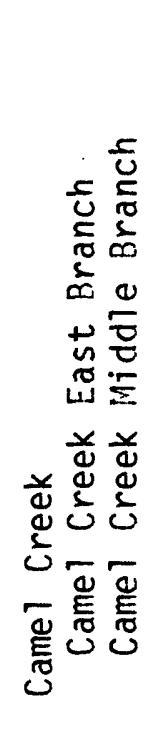 \\
\hline
\end{tabular}




\begin{tabular}{|c|c|c|c|c|c|c|c|}
\hline 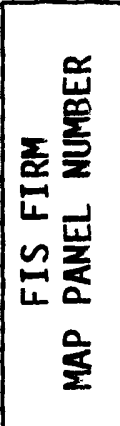 & & $\stackrel{\sim}{\sim}$ & 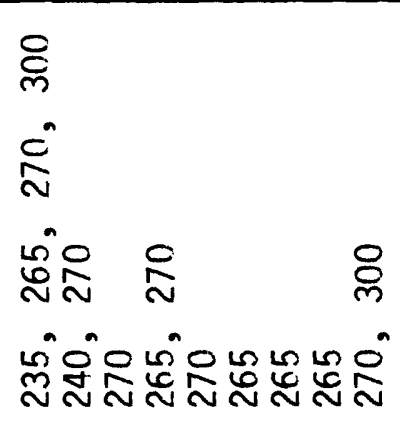 & 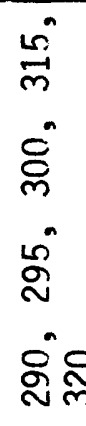 & 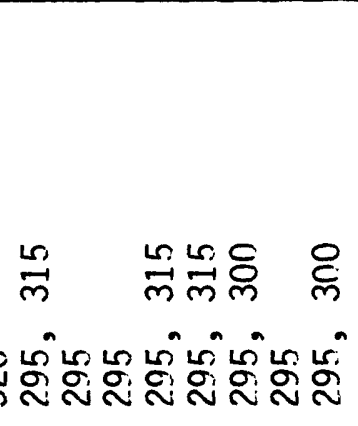 & & 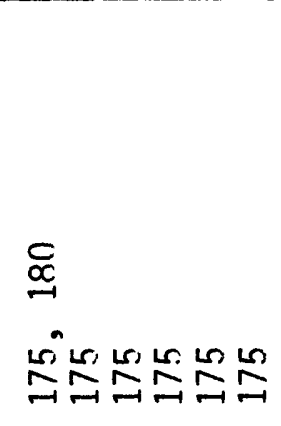 \\
\hline 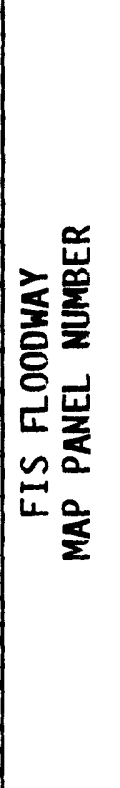 & & $\begin{array}{l}\stackrel{\infty}{=} \\
\stackrel{\Xi}{E} \\
\stackrel{E}{2}\end{array}$ & 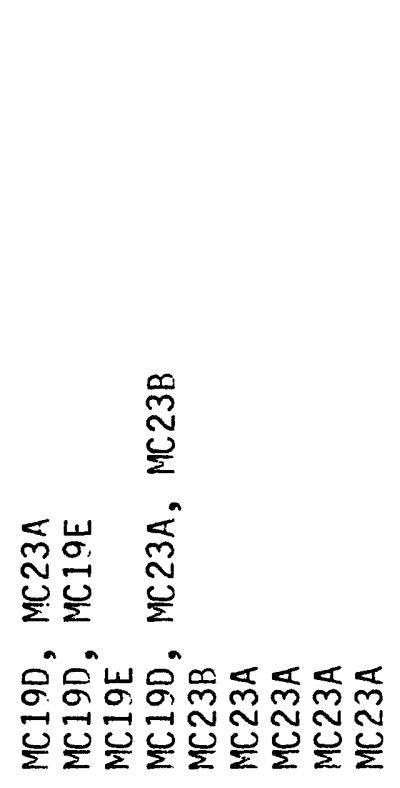 & 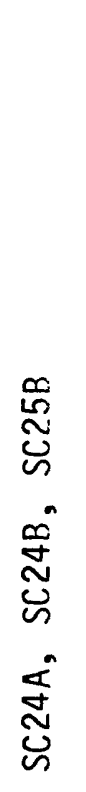 & 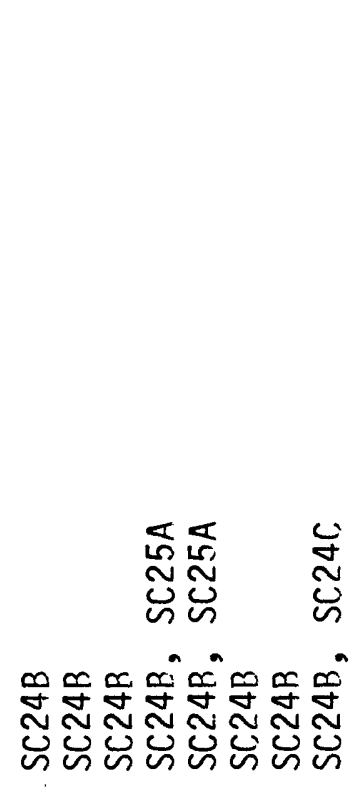 & & 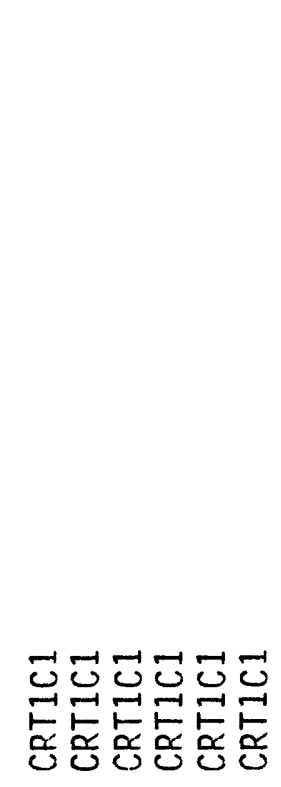 \\
\hline 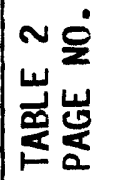 & & $\begin{array}{l}\text { ले } \\
\text { నे }\end{array}$ & চ & $\begin{array}{l}\dot{m} \\
\ddot{m}\end{array}$ & 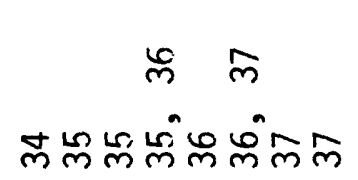 & & 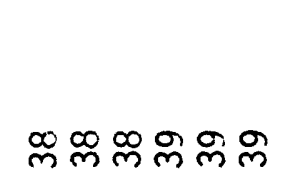 \\
\hline 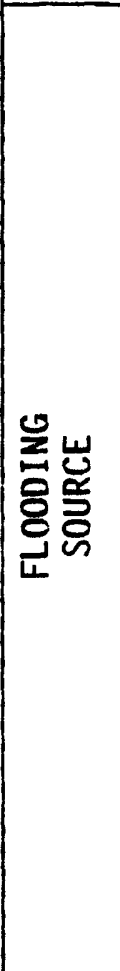 & 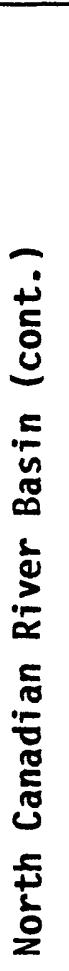 & 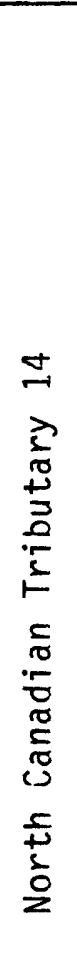 & 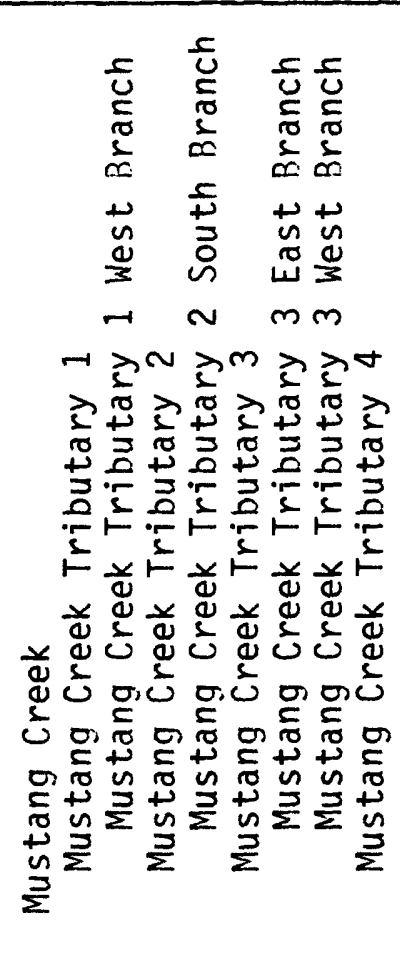 & 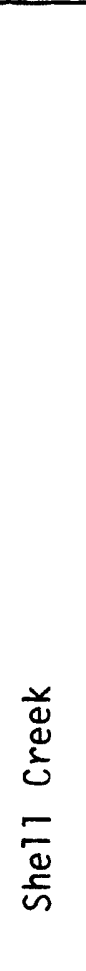 & 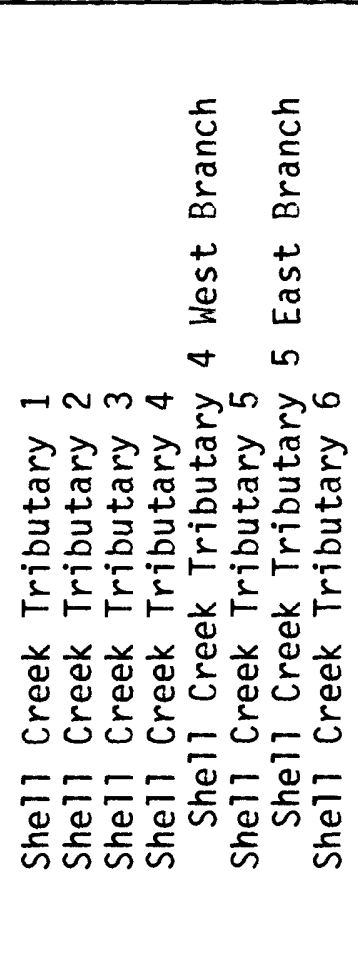 & 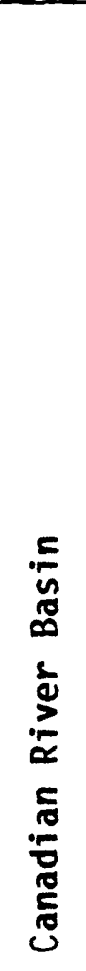 & 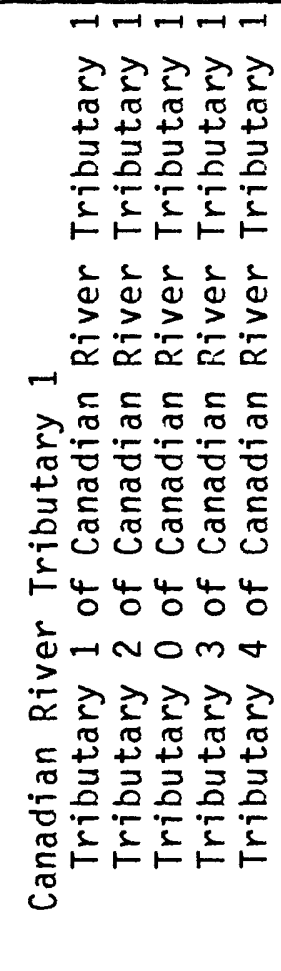 \\
\hline
\end{tabular}




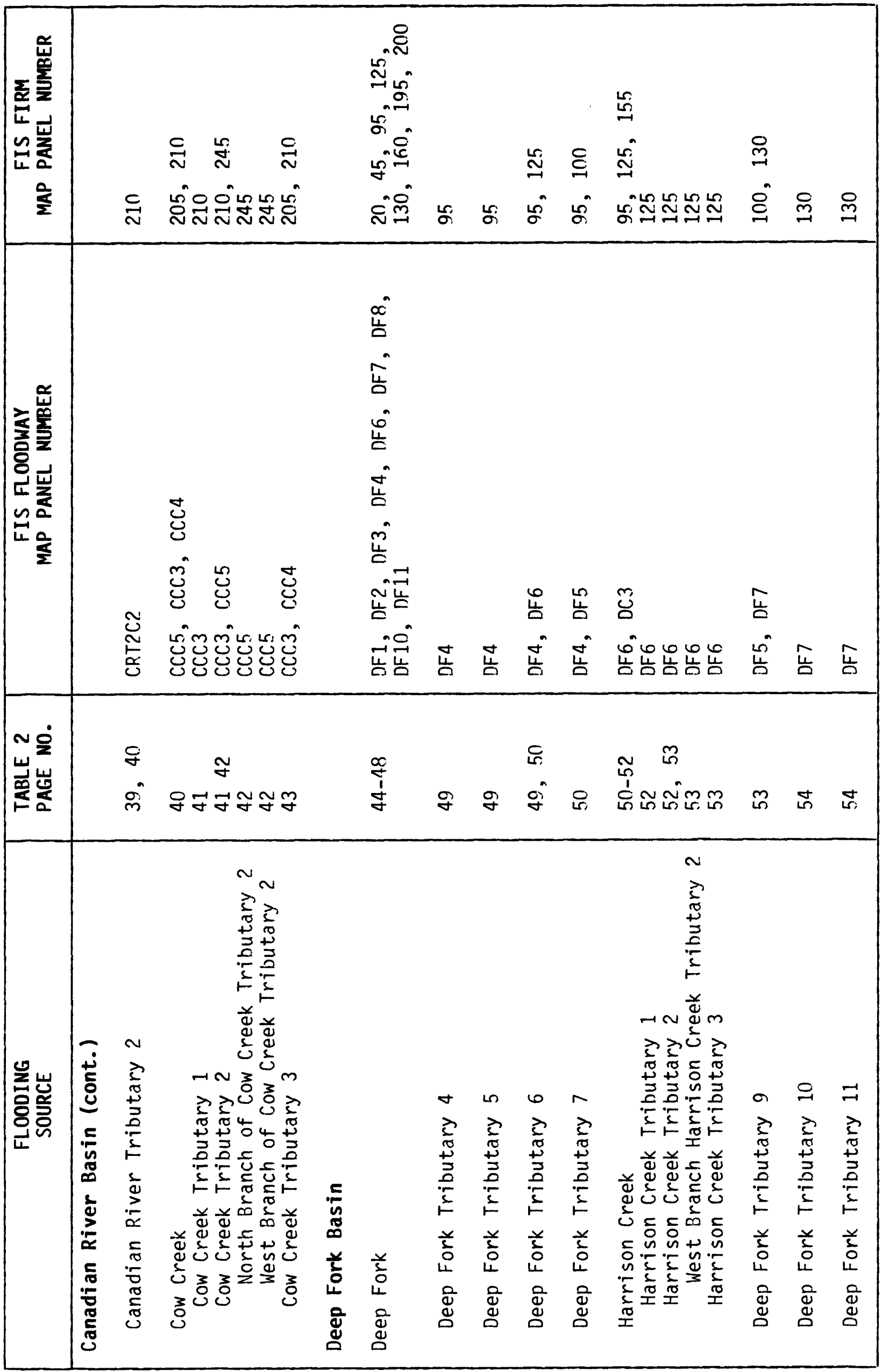




\begin{tabular}{|c|c|c|c|c|c|c|c|c|c|c|c|c|}
\hline 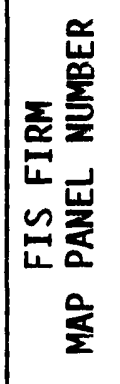 & & $\stackrel{\text { m }}{-}$ & 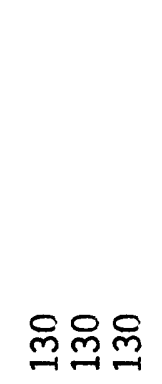 & 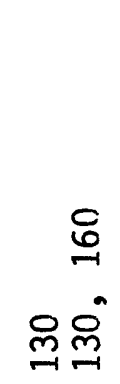 & $\begin{array}{l}\mathscr{C} \\
\stackrel{-1}{0} \\
\stackrel{\oplus}{-}\end{array}$ & $8 \underset{0}{8}$ & $\stackrel{8}{0}$ & 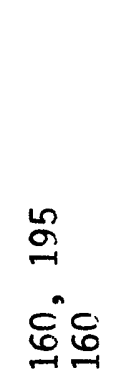 & $\begin{array}{l}\stackrel{2}{\sigma} \\
\stackrel{-1}{0} \\
\stackrel{0}{0}\end{array}$ & 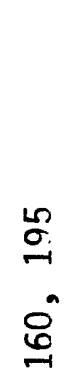 & & 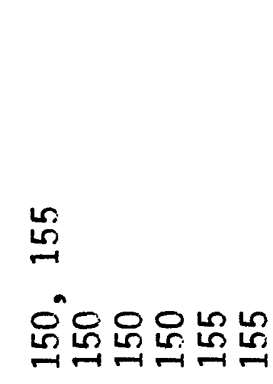 \\
\hline 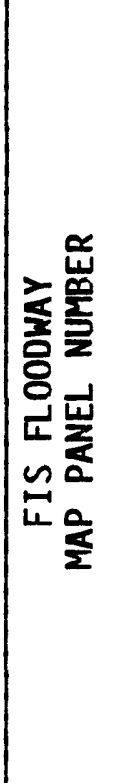 & & 㲒 & 台台台 & 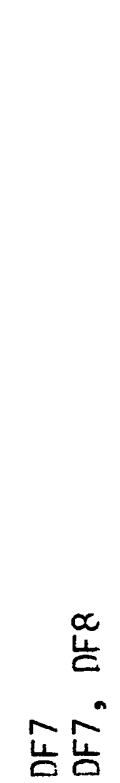 & 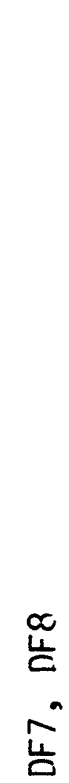 & $\begin{array}{l}\infty \infty \infty \\
\text { 岁告 }\end{array}$ & 䍃 & 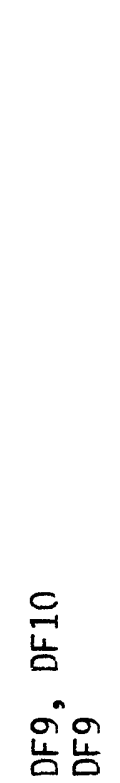 & $\begin{array}{l}\text { C } \\
\text { 岁 }\end{array}$ & 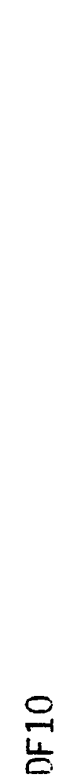 & & 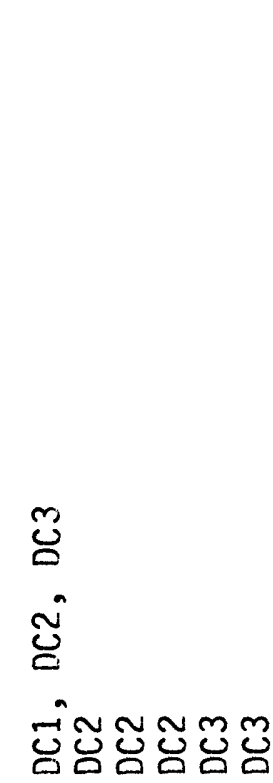 \\
\hline 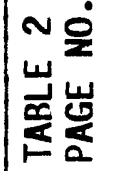 & & 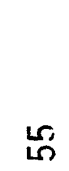 & 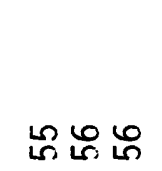 & 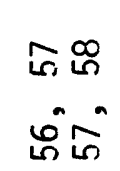 & $\infty$ & $\begin{array}{r}8 \\
\text { ㅇํำ }\end{array}$ & 8 & $\begin{array}{l}\overrightarrow{6} \\
\dot{8} \overrightarrow{6}\end{array}$ & $\vec{c}$ & $\widetilde{C}$ & & 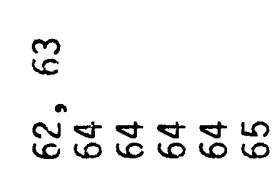 \\
\hline 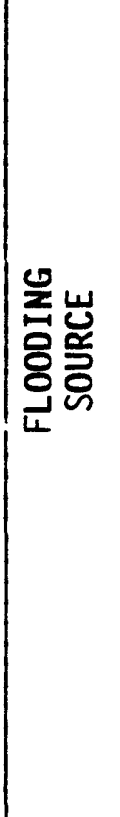 & 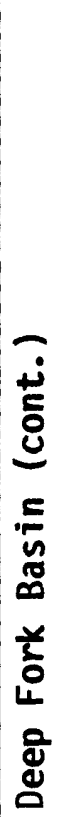 & 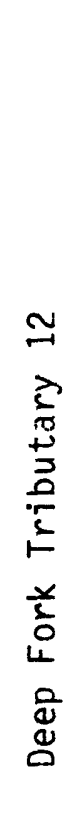 & 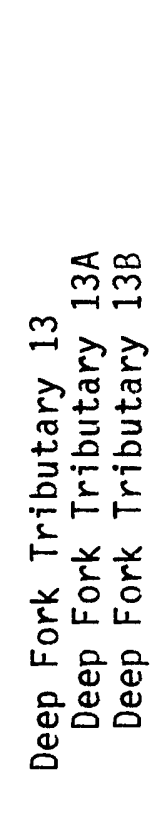 & 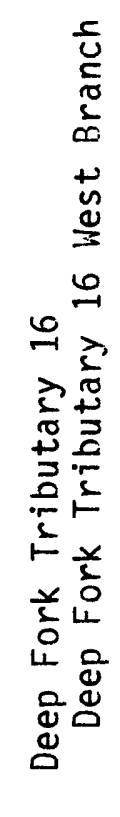 & 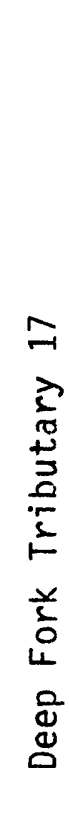 & 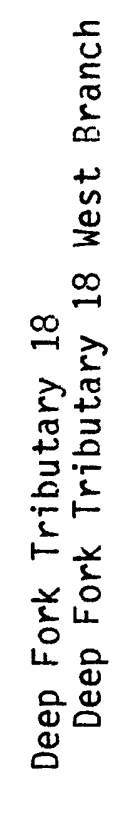 & 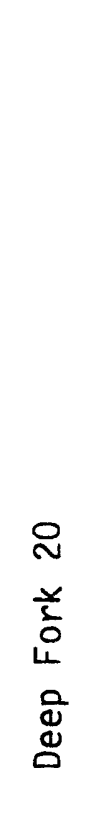 & 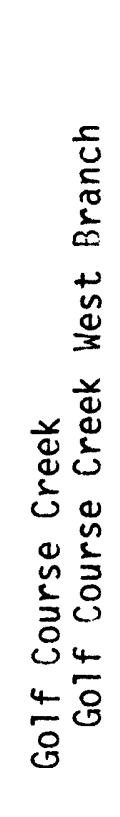 & 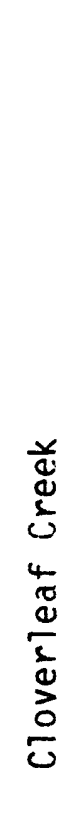 & 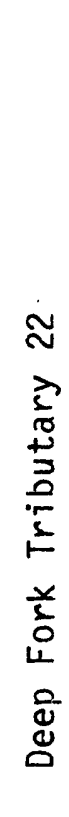 & 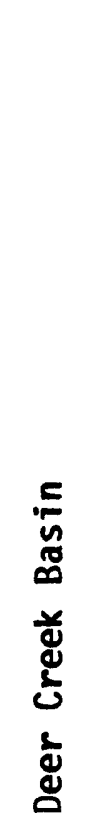 & 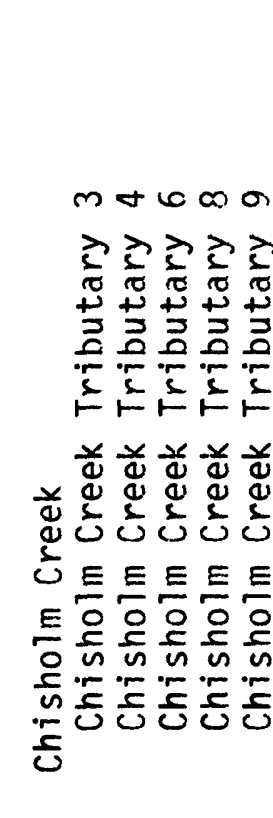 \\
\hline
\end{tabular}




\begin{tabular}{|c|c|c|c|c|c|c|c|c|c|c|c|c|c|c|c|}
\hline 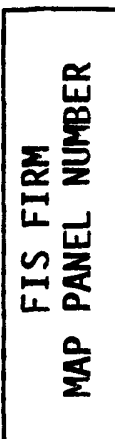 & & 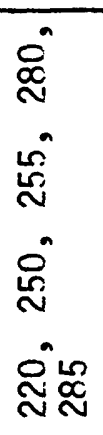 & $\stackrel{\infty}{\infty}$ & 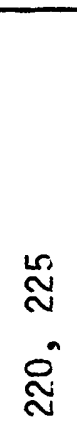 & $\begin{array}{l}\stackrel{\sim}{\approx} \\
\stackrel{\Sigma}{\approx} \approx\end{array}$ & 占 & 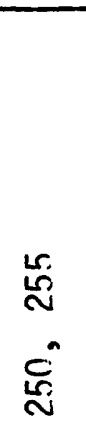 & 足 & $\stackrel{\sim}{\sim}$ & $\stackrel{\llcorner}{\stackrel{n}{\circ}}$ & $\begin{array}{l}\stackrel{c}{\infty} \\
\stackrel{\sim}{n} \\
\stackrel{n}{\sim}\end{array}$ & $\stackrel{0}{\stackrel{\sim}{\sim}}$ & \&్ల & $\stackrel{\llcorner}{\stackrel{D}{\sim}}$ & $\stackrel{\mathscr{N}}{\stackrel{\sim}{*}}$ \\
\hline 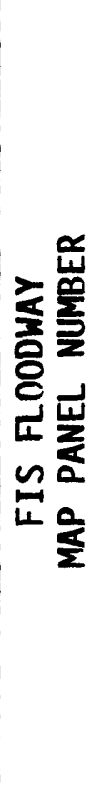 & & 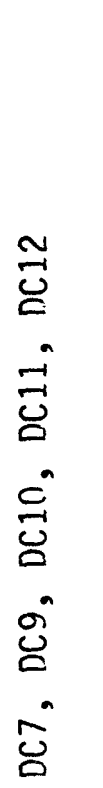 & 18 & $\begin{array}{l}\infty \\
\mathscr{0} \\
\tilde{0} \\
\tilde{0}\end{array}$ & 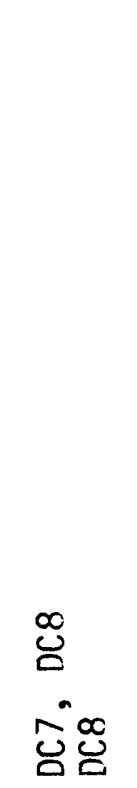 & $\stackrel{\circ}{\stackrel{ }{L}}$ & 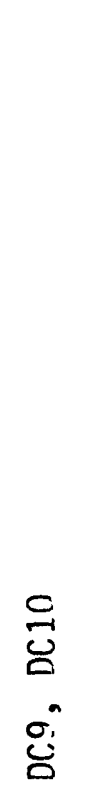 & $\stackrel{0}{\stackrel{\vec{d}}{\Delta}}$ & $\underset{\bar{U}}{\stackrel{c}{c}}$ & 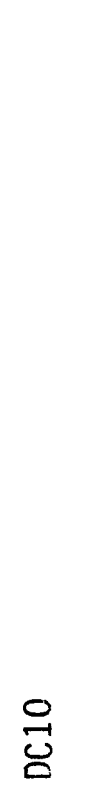 & 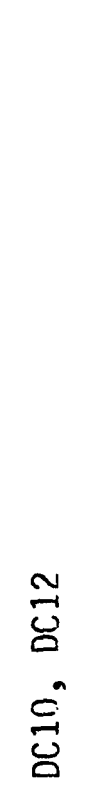 & $\overrightarrow{\bar{\Delta}}$ & 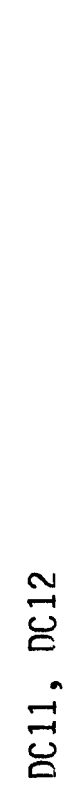 & $\stackrel{\sim}{\vec{\Delta}}$ & $\stackrel{\widetilde{J}}{\widetilde{D}}$ \\
\hline 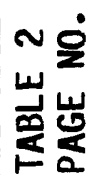 & & $\begin{array}{l}0 \\
0 \\
0\end{array}$ & 8 & $\begin{array}{l}\mathscr{\infty} \\
1 \\
1 \\
0 \\
0\end{array}$ & 08 & 8 & 8 & $\begin{array}{l}8 \\
8 \\
8\end{array}$ & $\stackrel{R}{R}$ & $\stackrel{0}{ }$ & $\vec{r}$ & $\cong$ & $\approx$ & $\approx$ & $\stackrel{2}{n}$ \\
\hline 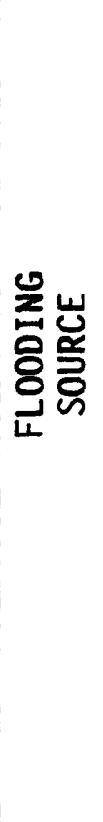 & 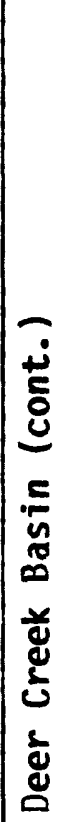 & 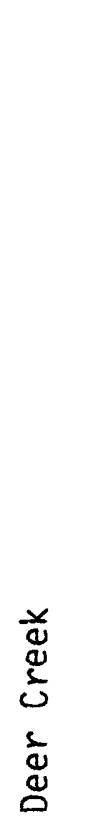 & 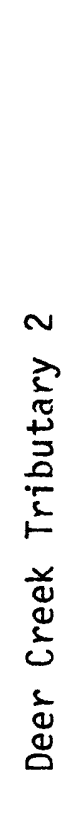 & 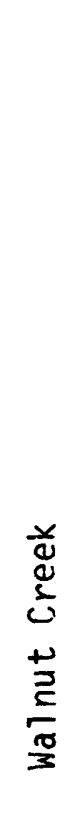 & 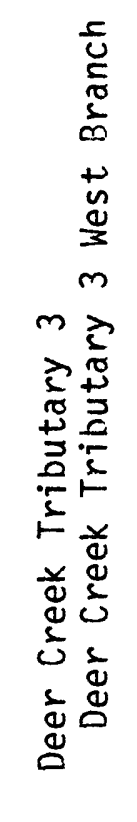 & 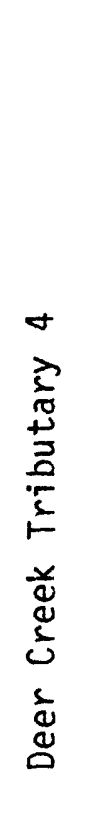 & 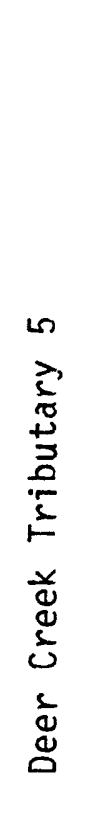 & 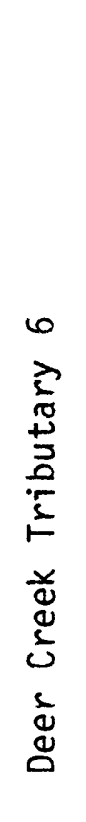 & 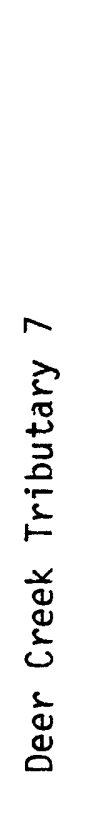 & 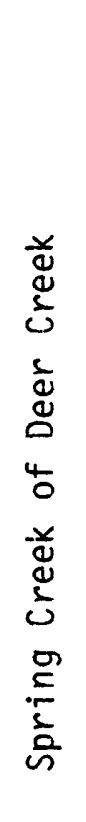 & 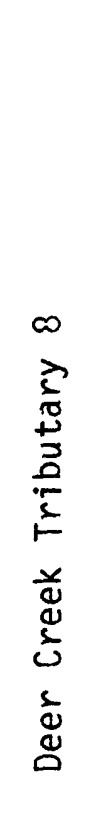 & 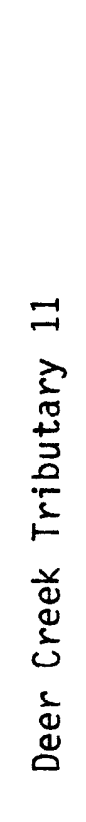 & 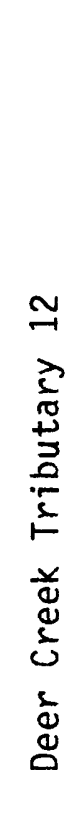 & 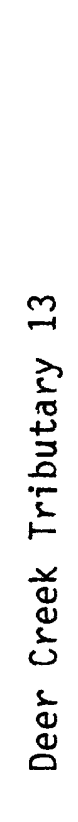 & 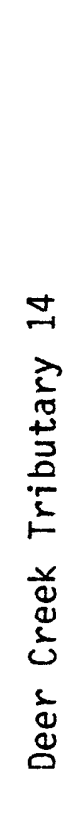 \\
\hline
\end{tabular}




\begin{tabular}{|c|c|c|c|c|c|c|c|c|c|c|c|c|}
\hline 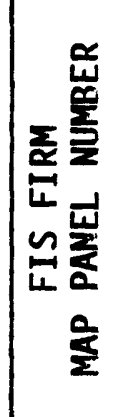 & & 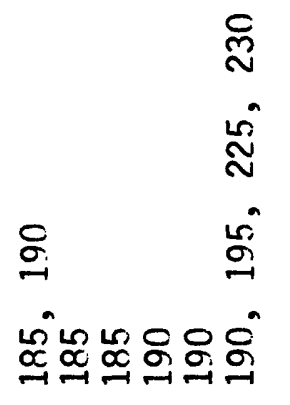 & 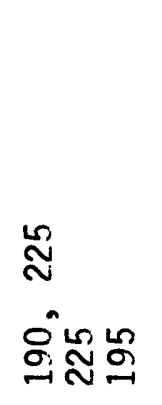 & & $\begin{array}{l}8 \\
80 \\
8 \\
8 \\
8^{n}\end{array}$ & שֶׁ & 亗 & $\begin{array}{l}8 \\
\tilde{n}^{n}\end{array}$ & 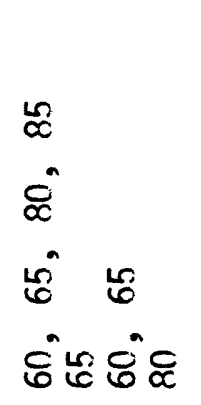 & $\mathbb{8}$ & $\mathbb{E}$ & 8 \\
\hline 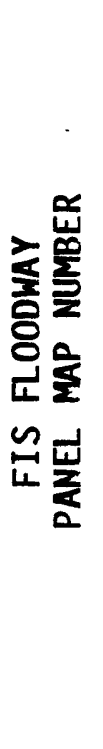 & & 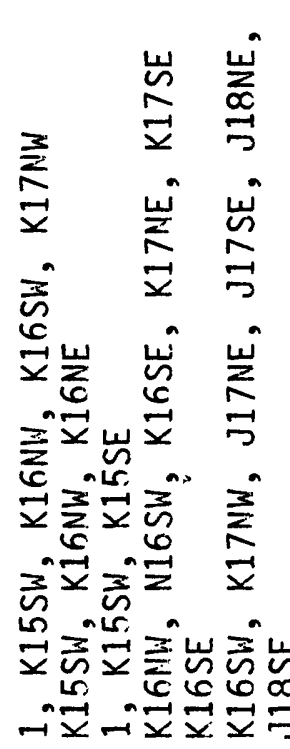 & 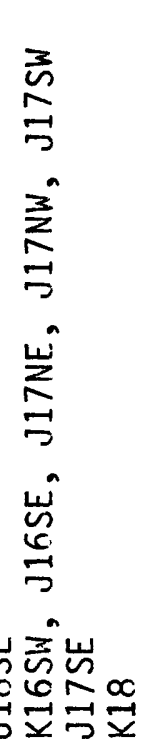 & & 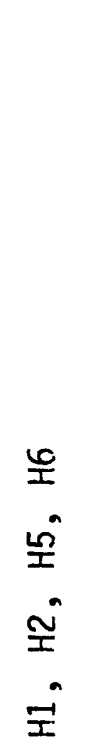 & $\simeq$ & 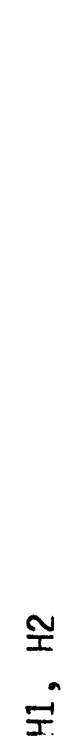 & 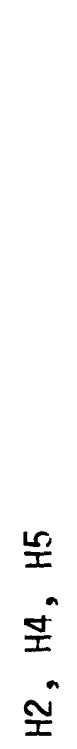 & 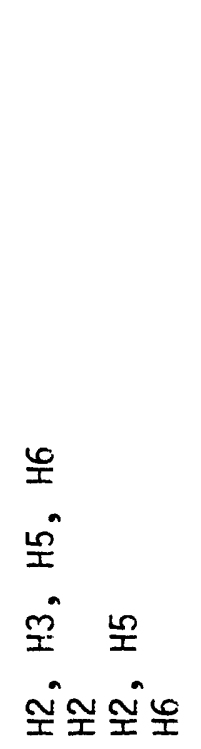 & 占 & 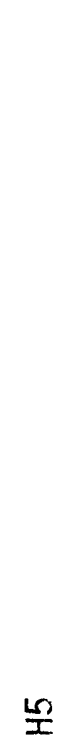 & 높 \\
\hline 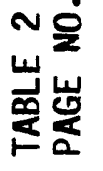 & & 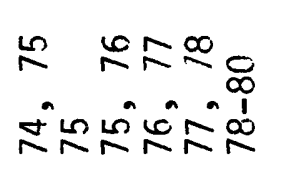 & $\begin{array}{l}\vec{\infty} \\
\vec{\infty} \tilde{\infty} \infty\end{array}$ & & $\begin{array}{l}\infty \\
1 \\
1 \\
\infty\end{array}$ & $\stackrel{\infty}{\infty}$ & $\stackrel{\llcorner}{\infty}$ & $\begin{array}{l}\infty \\
\infty \\
\infty\end{array}$ & $\begin{array}{l}\hat{\infty} \\
\mathscr{\infty}_{\infty} \hat{\infty} \hat{\infty} \hat{\infty}\end{array}$ & $\stackrel{\infty}{\propto}$ & $\underset{\infty}{\infty}$ & $\stackrel{\infty}{\propto}$ \\
\hline 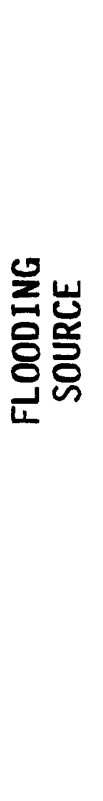 & 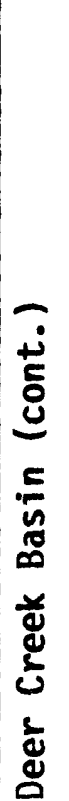 & 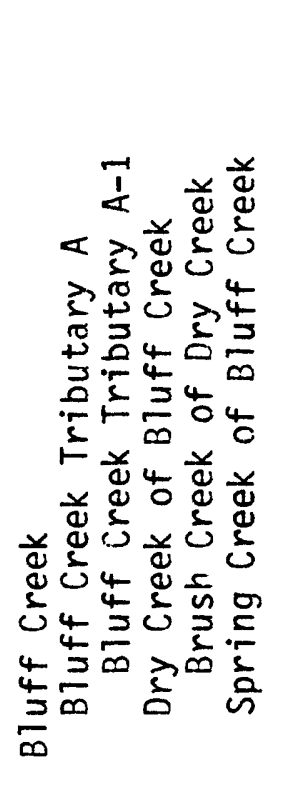 & 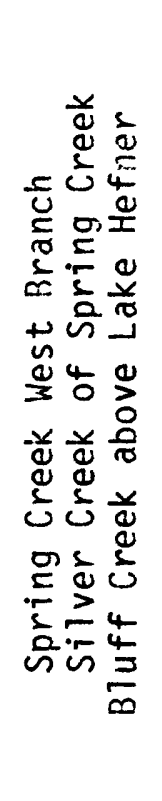 & 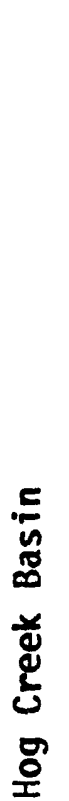 & 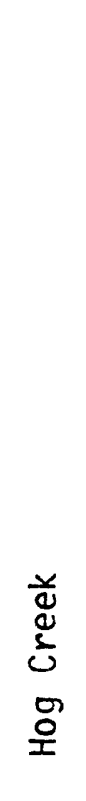 & 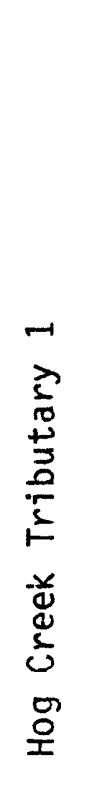 & 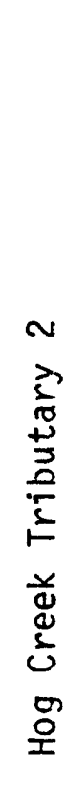 & 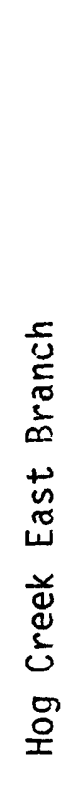 & 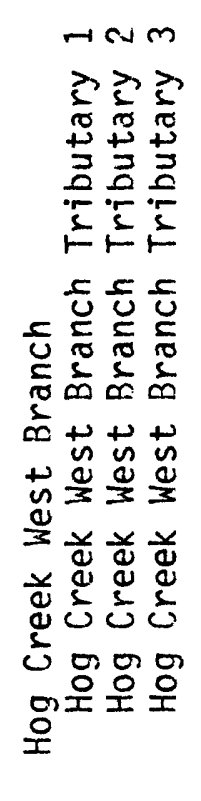 & 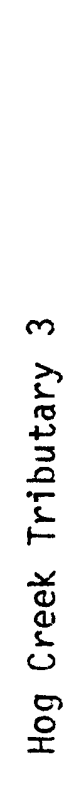 & 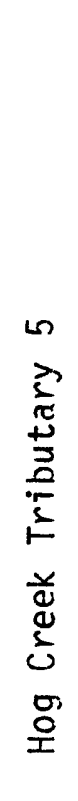 & 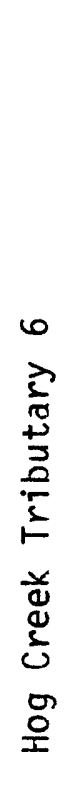 \\
\hline
\end{tabular}




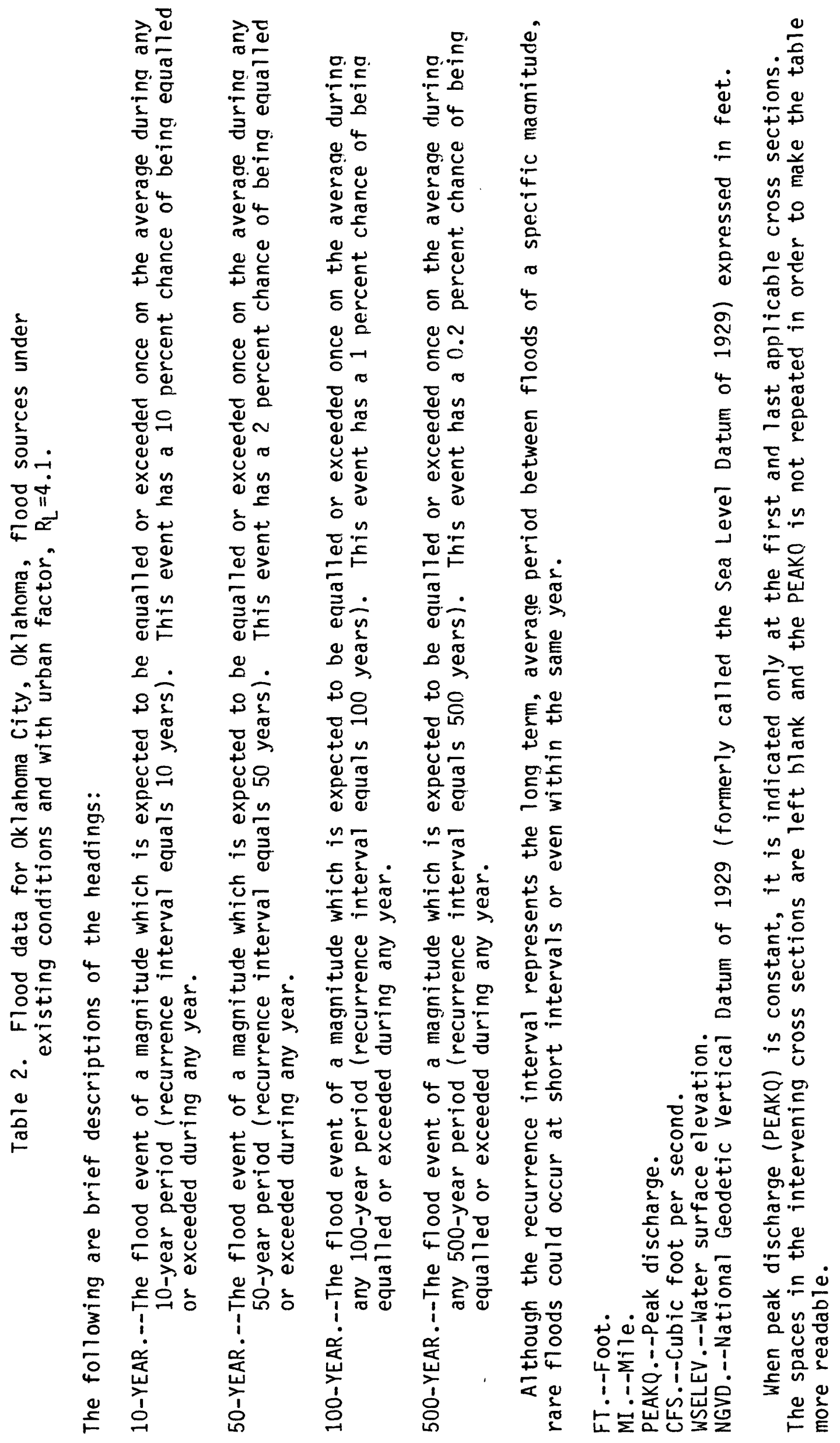




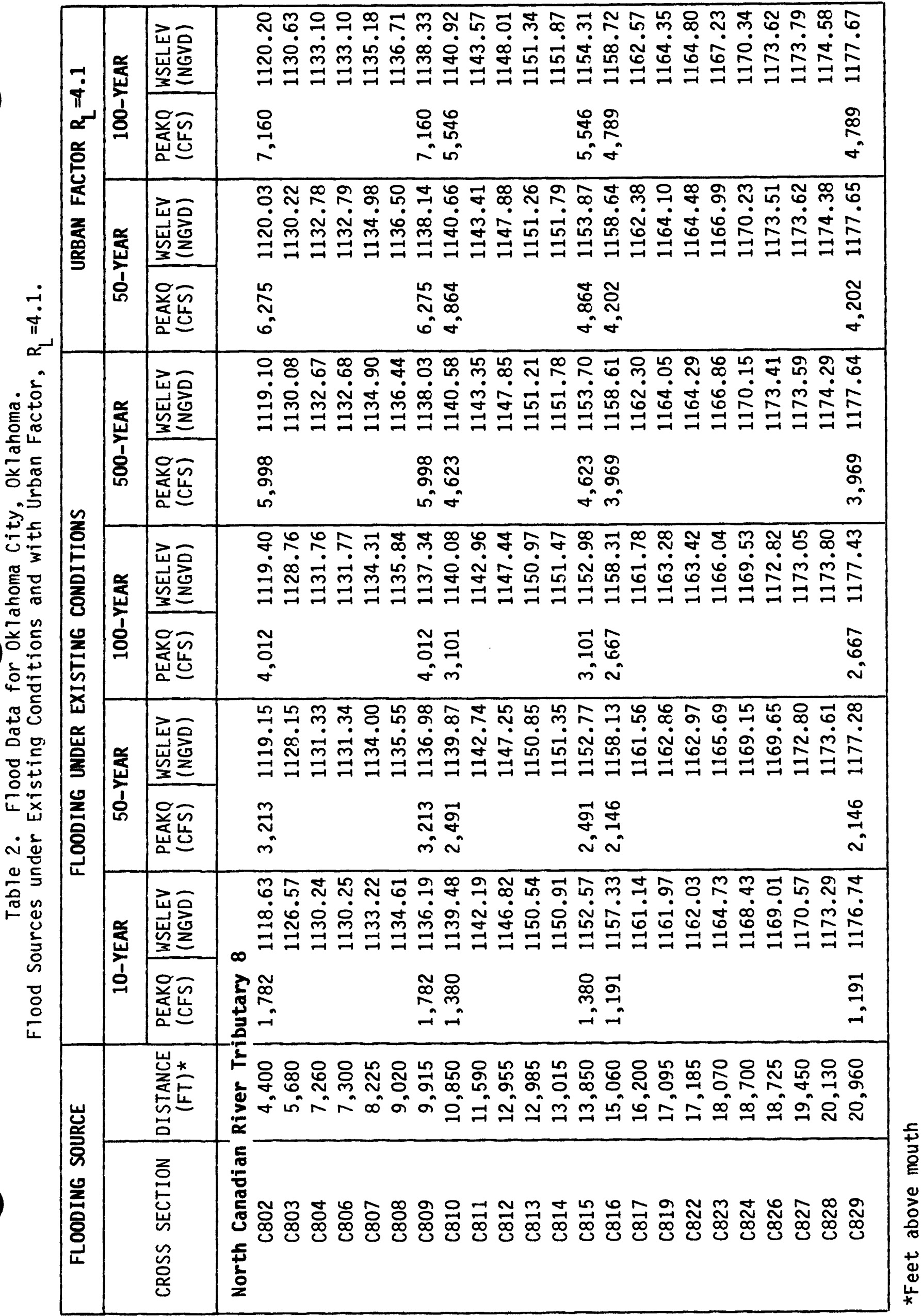




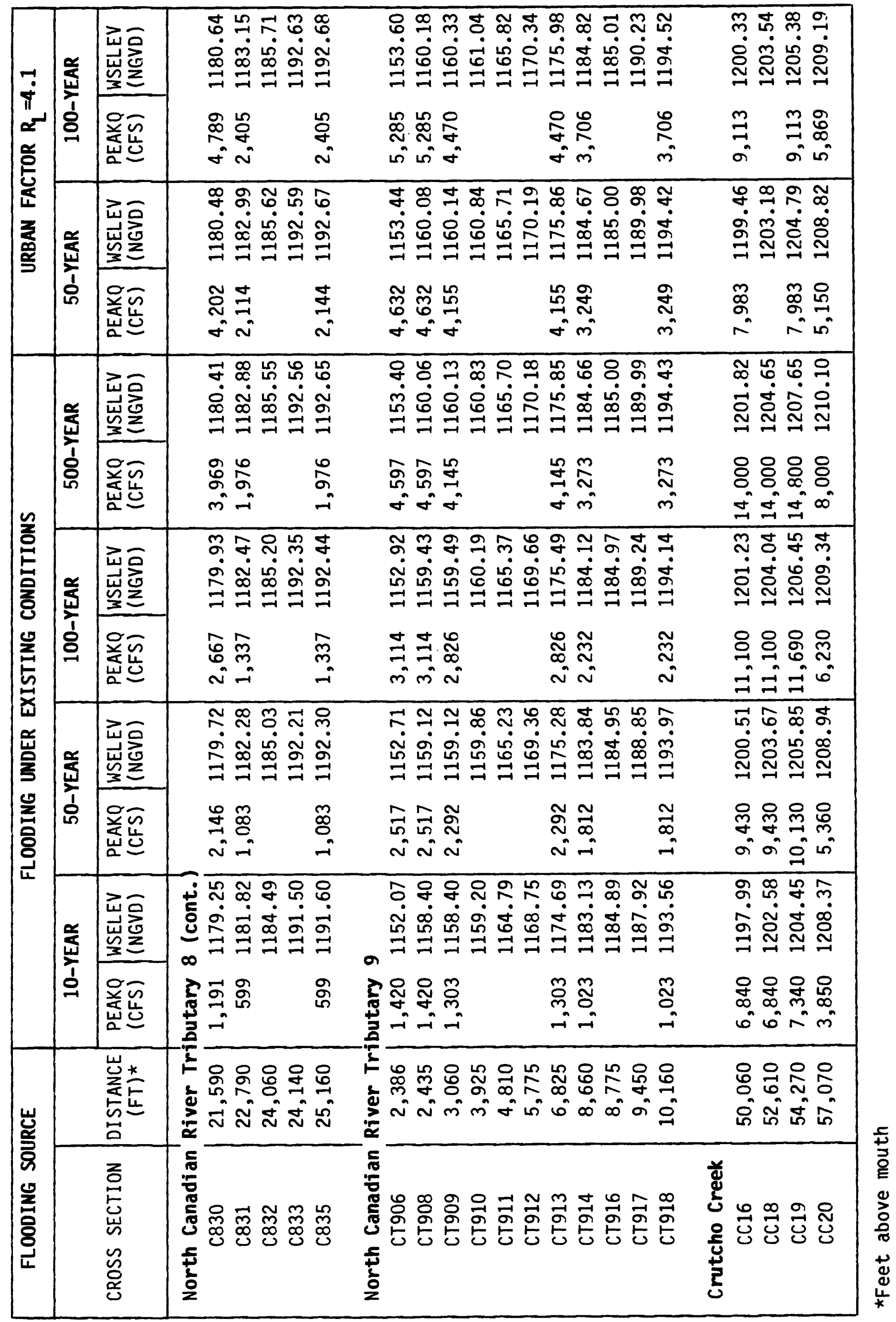




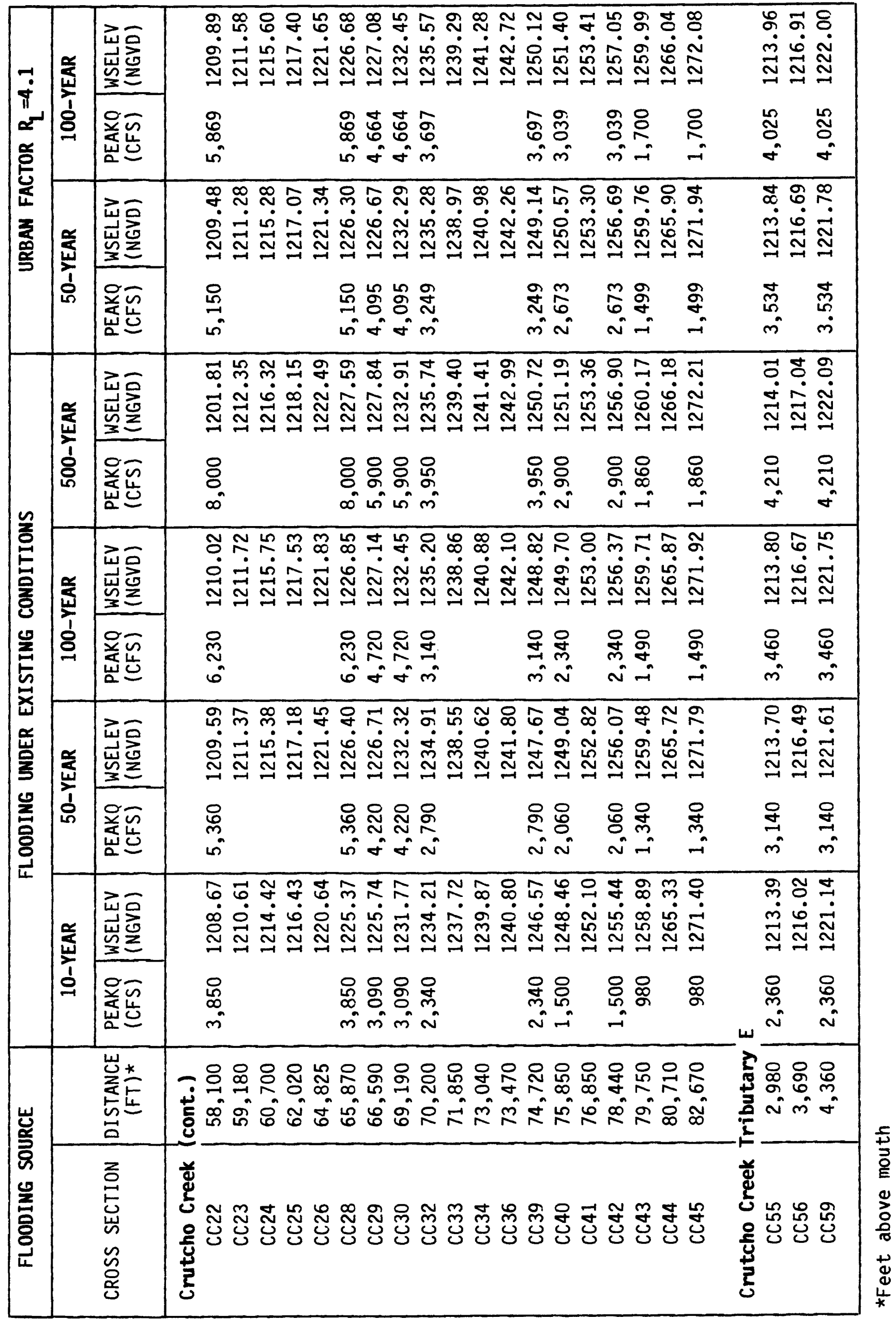




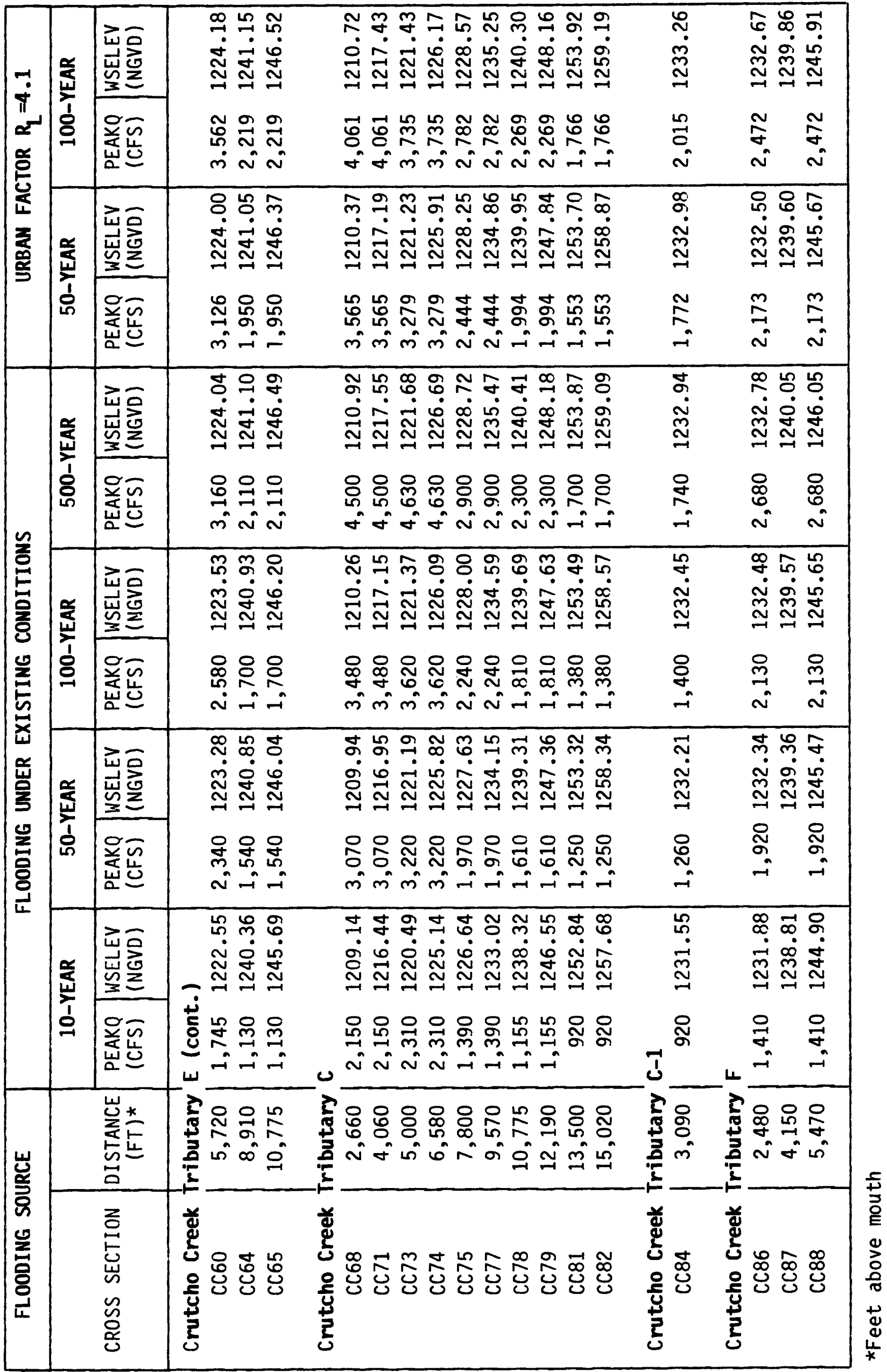




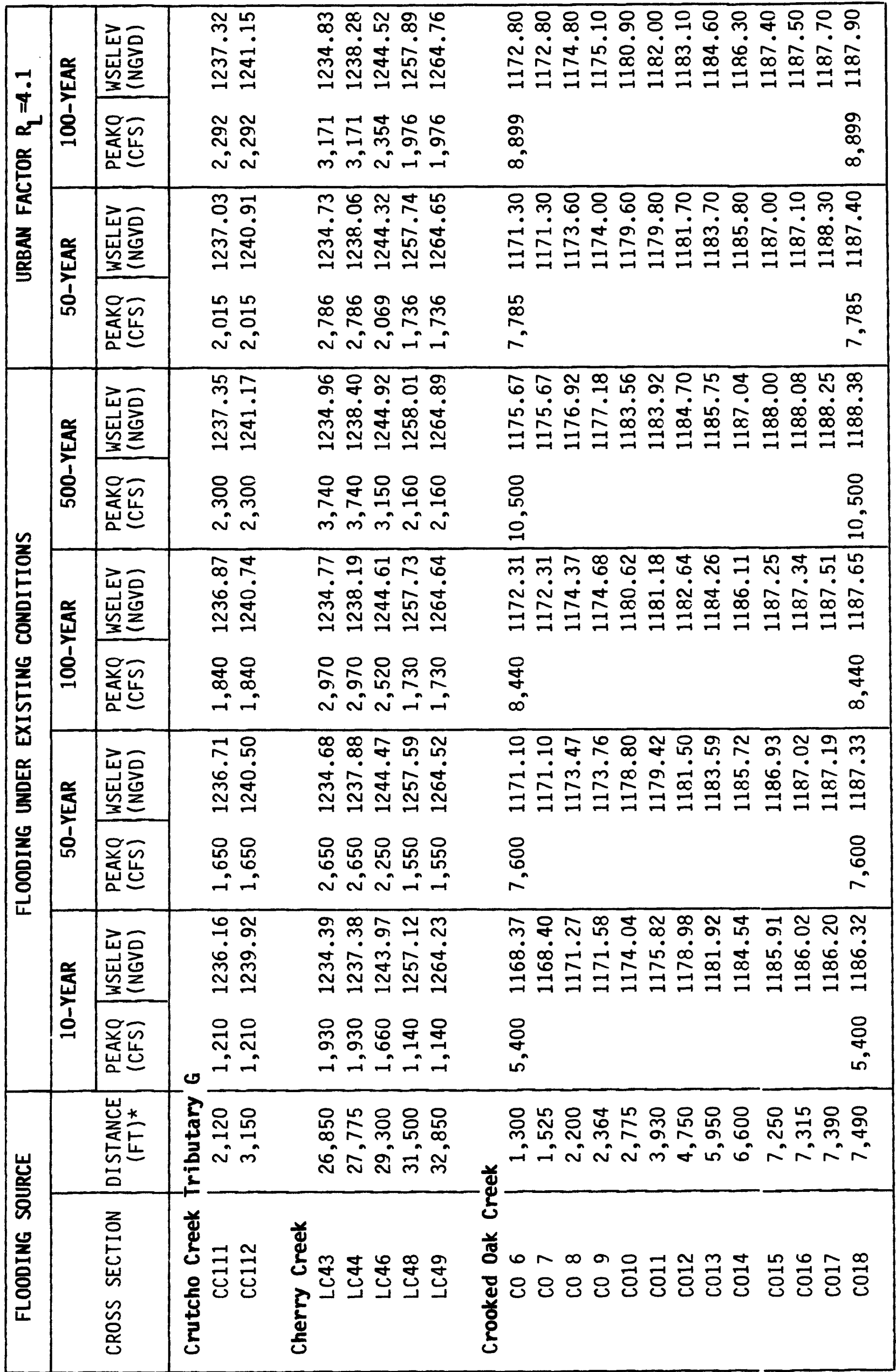




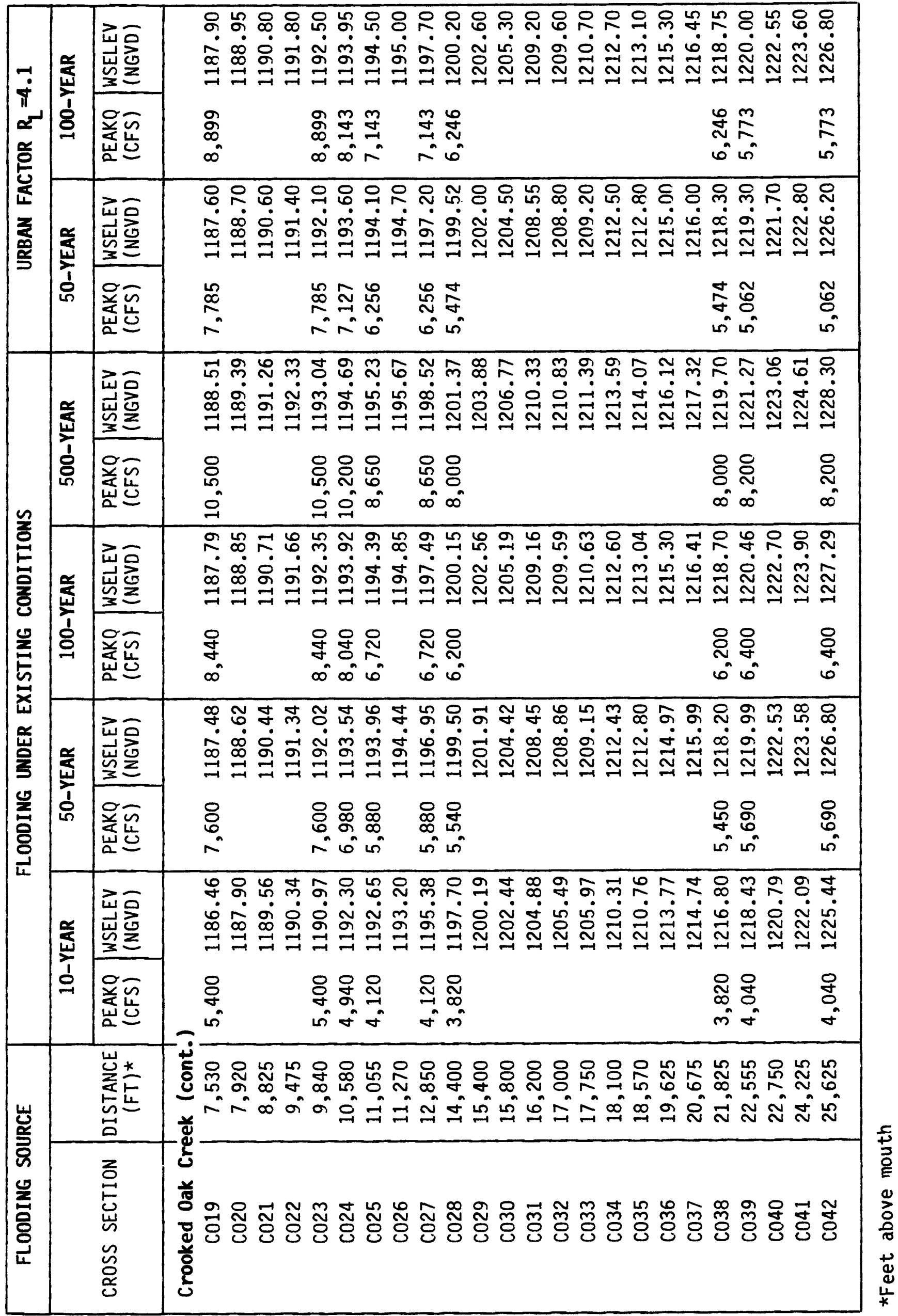




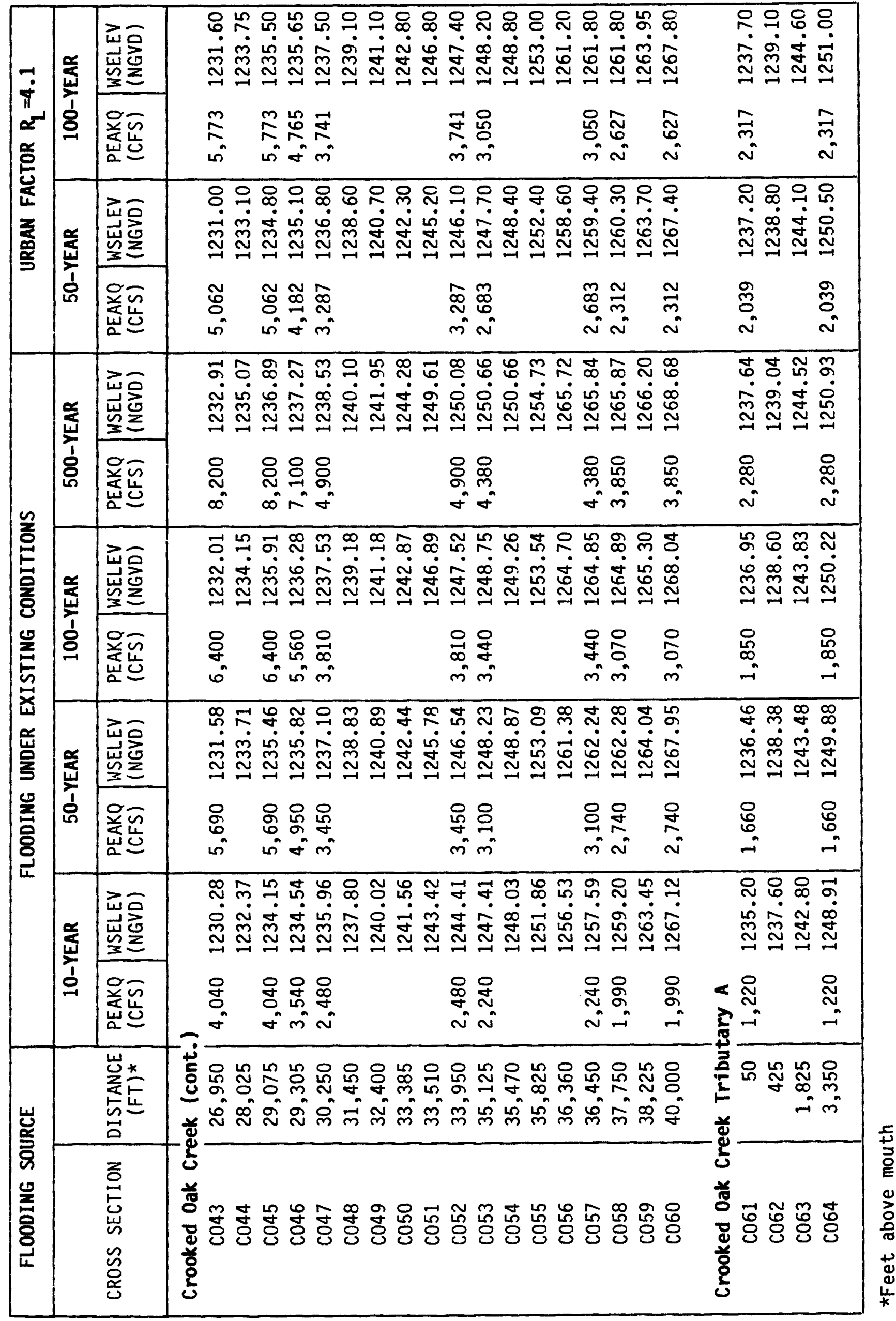




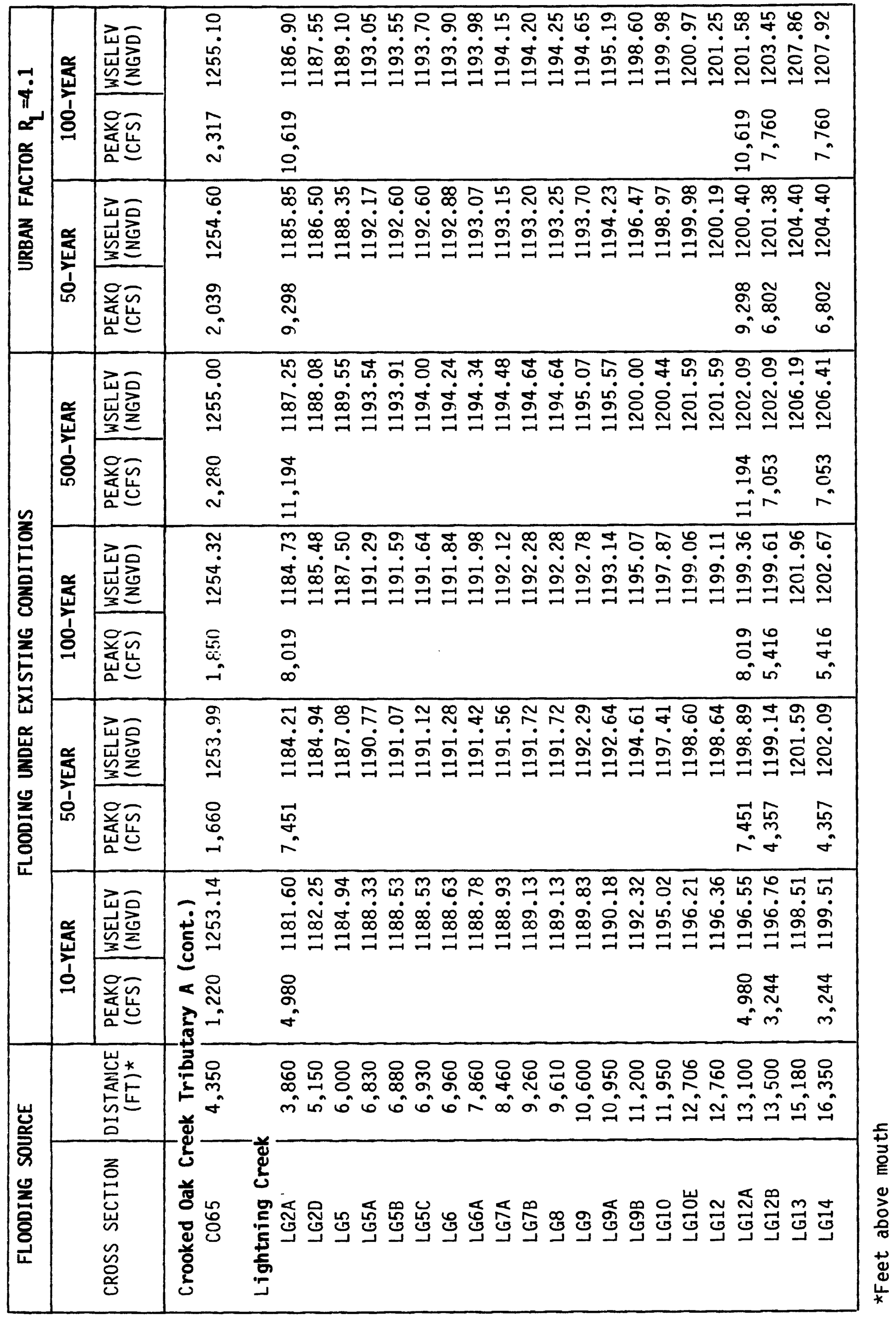




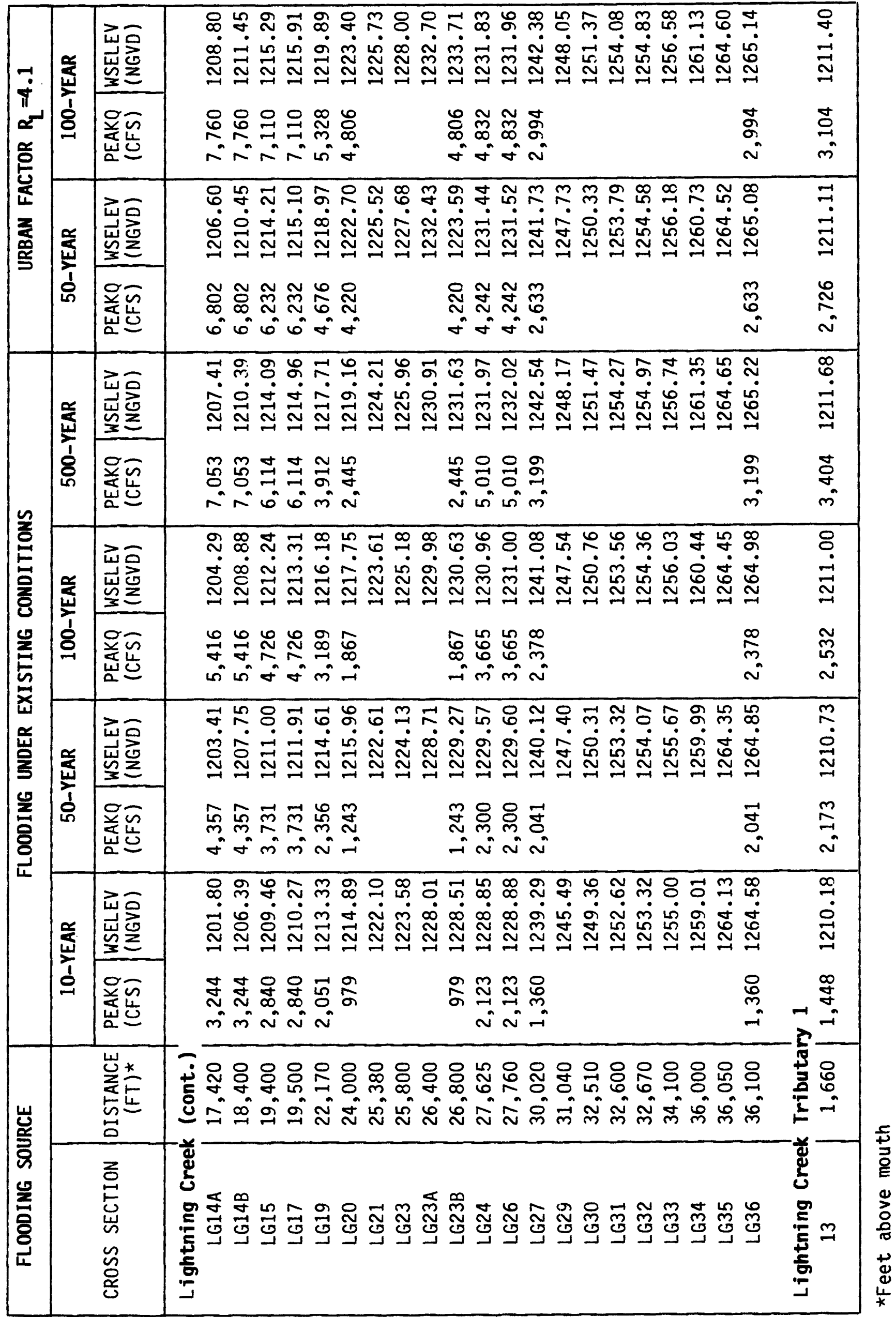




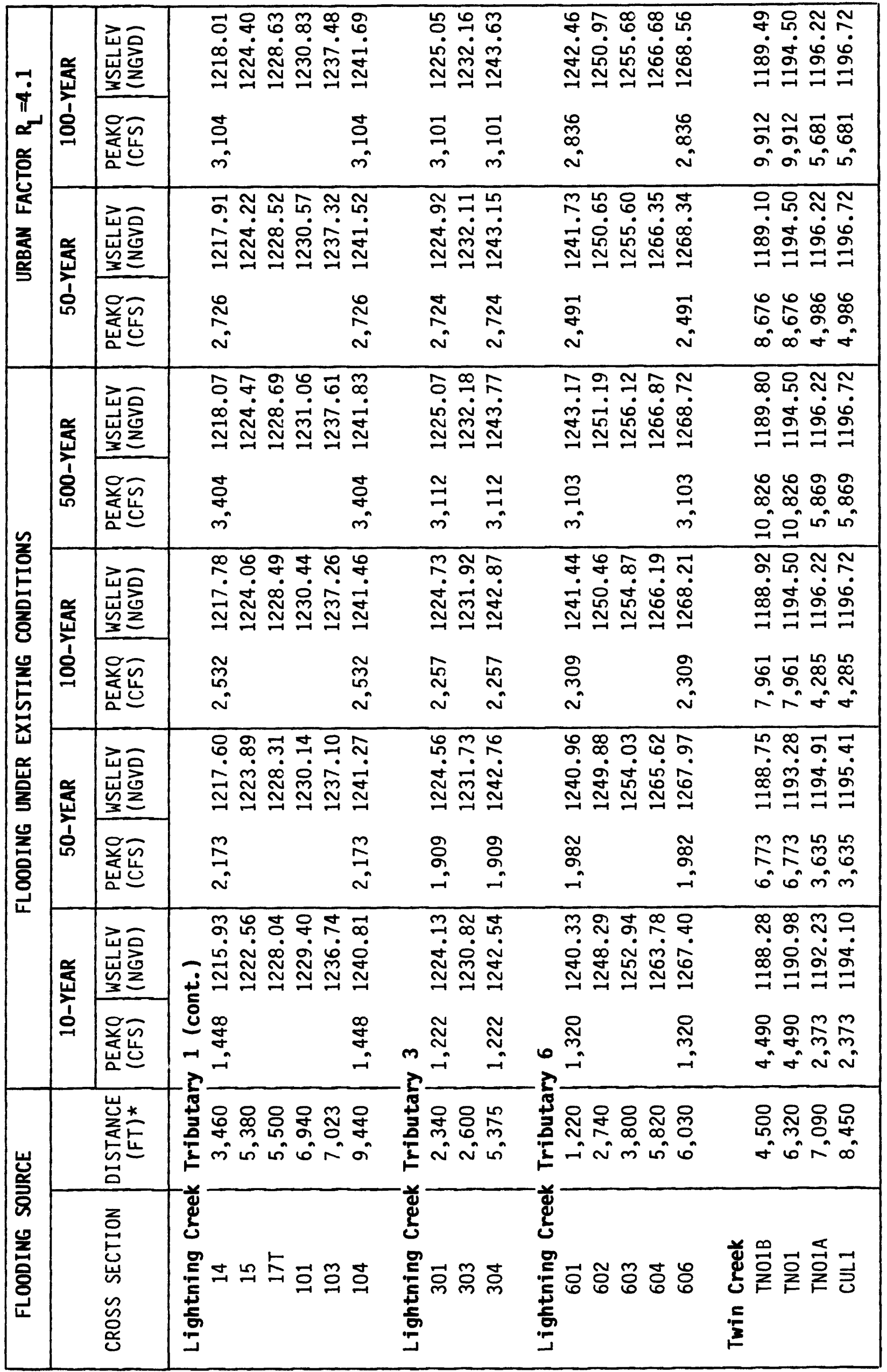




\begin{tabular}{|c|c|c|c|c|c|c|}
\hline \multirow{4}{*}{ 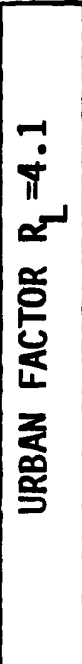 } & \multirow{2}{*}{$\frac{0}{\frac{2}{5}}$} & \multirow{2}{*}{ 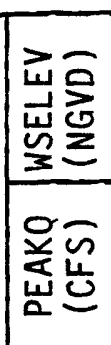 } & 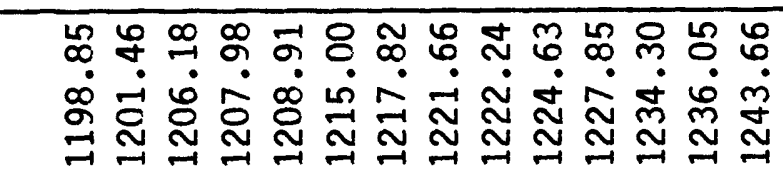 & \multicolumn{3}{|c|}{ 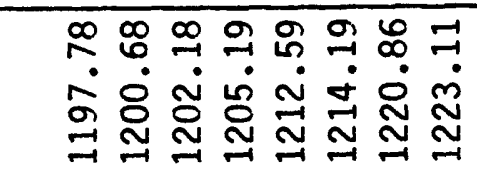 } \\
\hline & & & 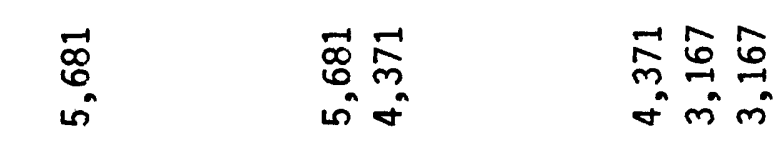 & 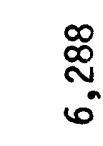 & 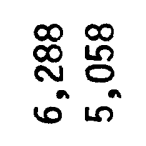 & $\begin{array}{l}\infty \\
\stackrel{\infty}{0} \\
\tilde{n}^{n}\end{array}$ \\
\hline & \multirow{2}{*}{$\mid \begin{array}{c}\frac{0}{5} \\
\frac{1}{1} \\
0 \\
0\end{array}$} & 崖㤩 & 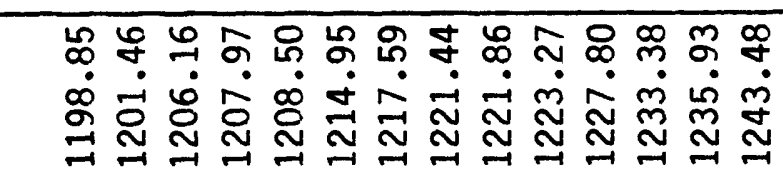 & \multicolumn{3}{|c|}{ 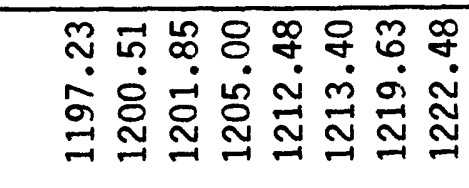 } \\
\hline & & 道苍 & 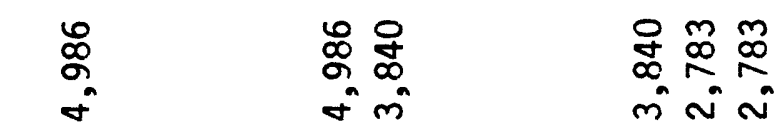 & $\begin{array}{l}\frac{n}{n} \\
\text { مी }\end{array}$ & 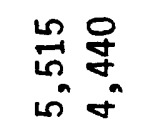 & $\begin{array}{l}\text { \& } \\
\text { \& }\end{array}$ \\
\hline \multirow{8}{*}{ 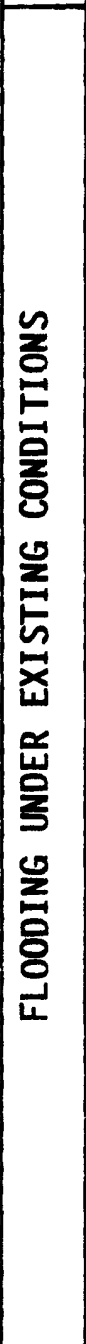 } & \multirow{2}{*}{ 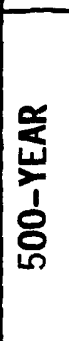 } & 岂㤩 & 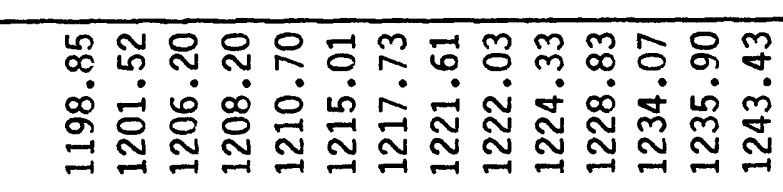 & \multicolumn{3}{|c|}{ 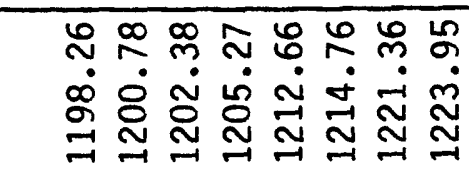 } \\
\hline & & 荃苍 & 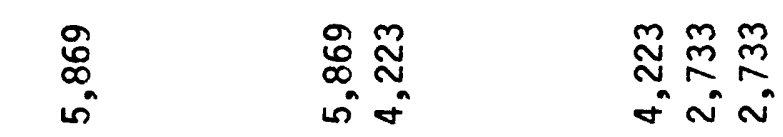 & $\begin{array}{l}\text { ने } \\
6\end{array}$ & 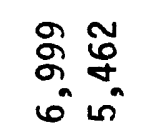 & 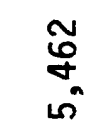 \\
\hline & \multirow{2}{*}{ 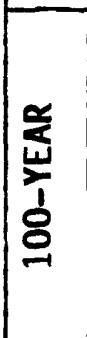 } & 崖 & 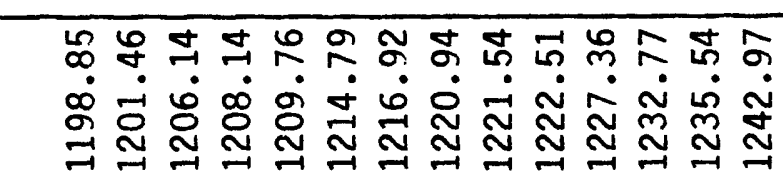 & \multicolumn{3}{|c|}{ 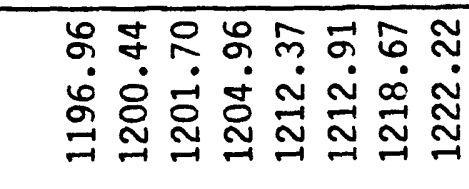 } \\
\hline & & 送炰 & 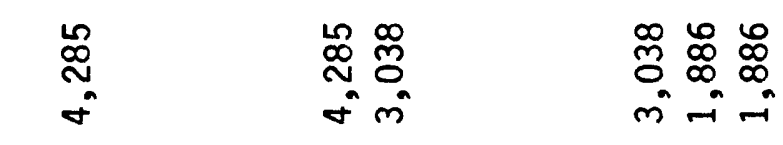 & $\stackrel{0}{\stackrel{0}{\sim}}$ & 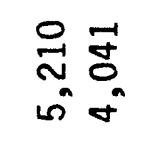 & $\begin{array}{l}F \\
\dot{\sigma}\end{array}$ \\
\hline & \multirow{2}{*}{ 立 } & 岂㤩 & 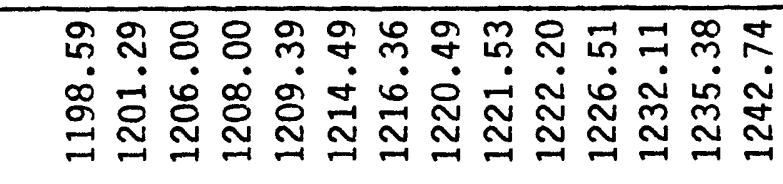 & \multicolumn{3}{|c|}{ 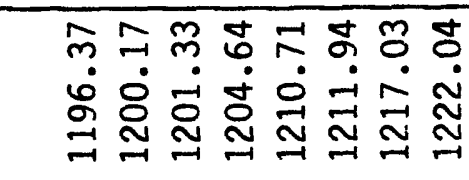 } \\
\hline & & 羙芯 & 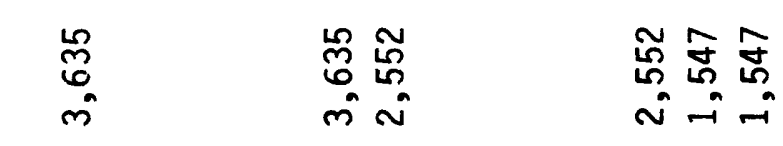 & $\begin{array}{l}8 \\
\& \\
\dot{\sigma}\end{array}$ & 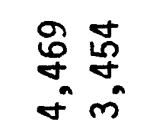 & \\
\hline & \multirow{2}{*}{$\underset{\frac{1}{4}}{\frac{\alpha}{1}}$} & 岂 & 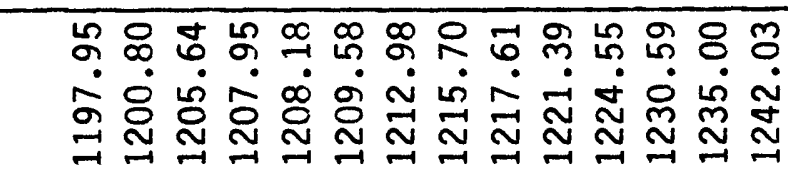 & \multicolumn{3}{|c|}{ 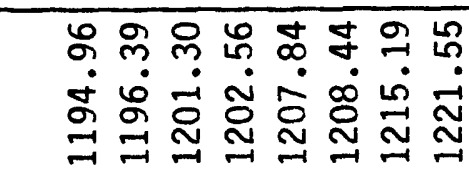 } \\
\hline & & 房总 & 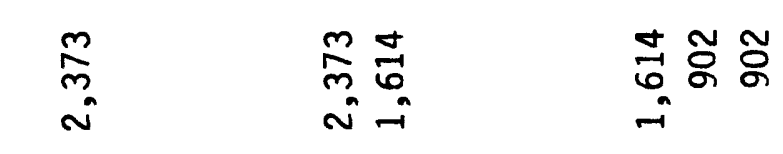 & $\begin{array}{l}\stackrel{2}{0} \\
\text { m }\end{array}$ & 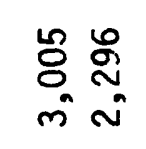 & ని \\
\hline \multirow{2}{*}{ 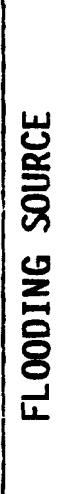 } & & 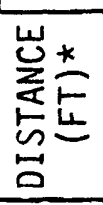 & 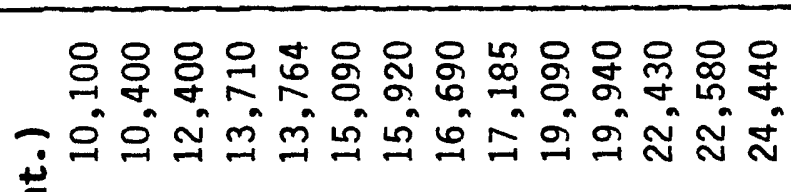 & \multicolumn{3}{|c|}{ 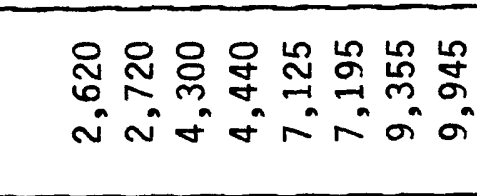 } \\
\hline & & 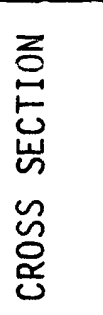 & 总 & 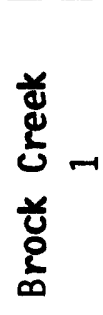 & & \\
\hline
\end{tabular}




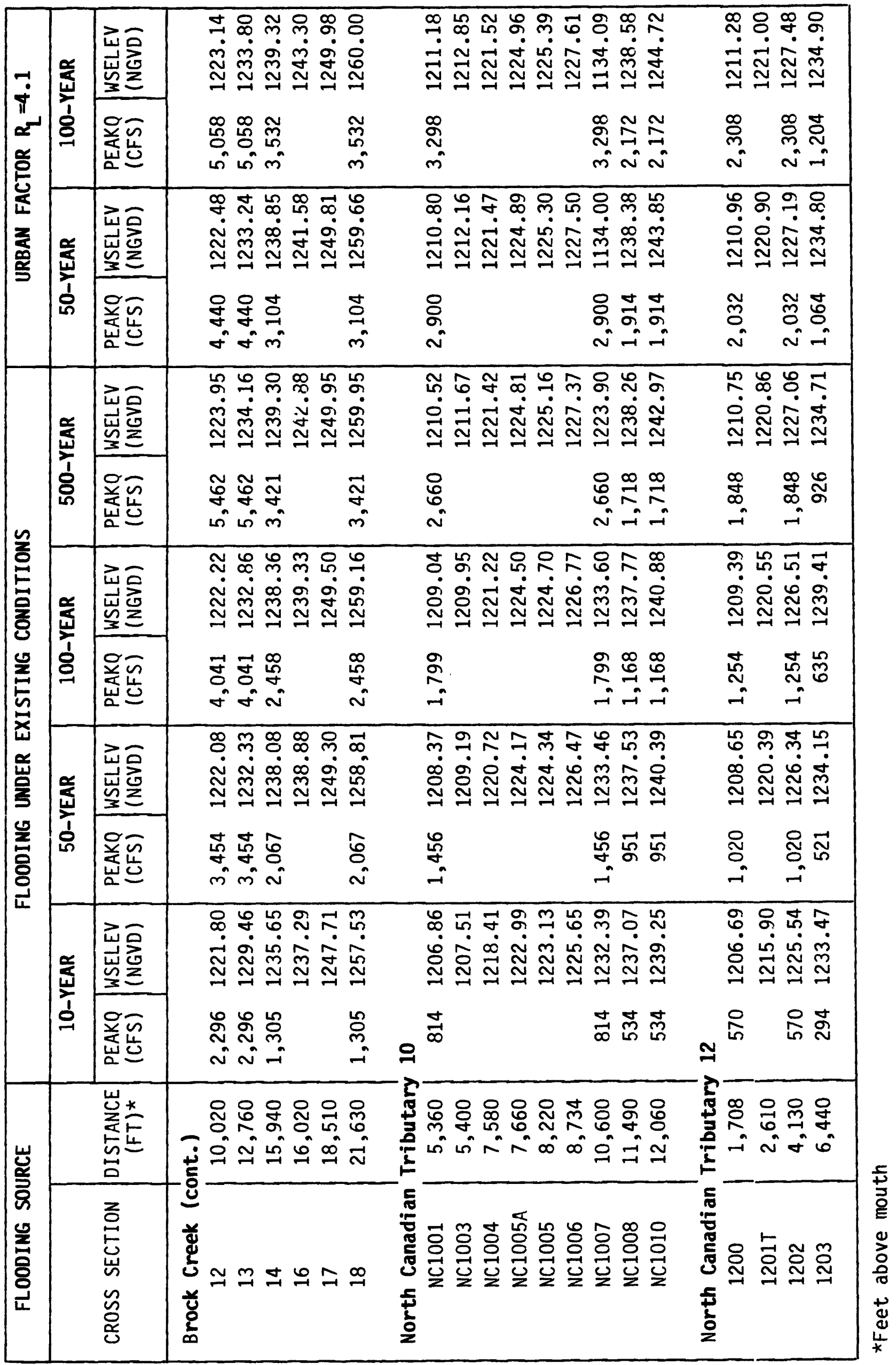




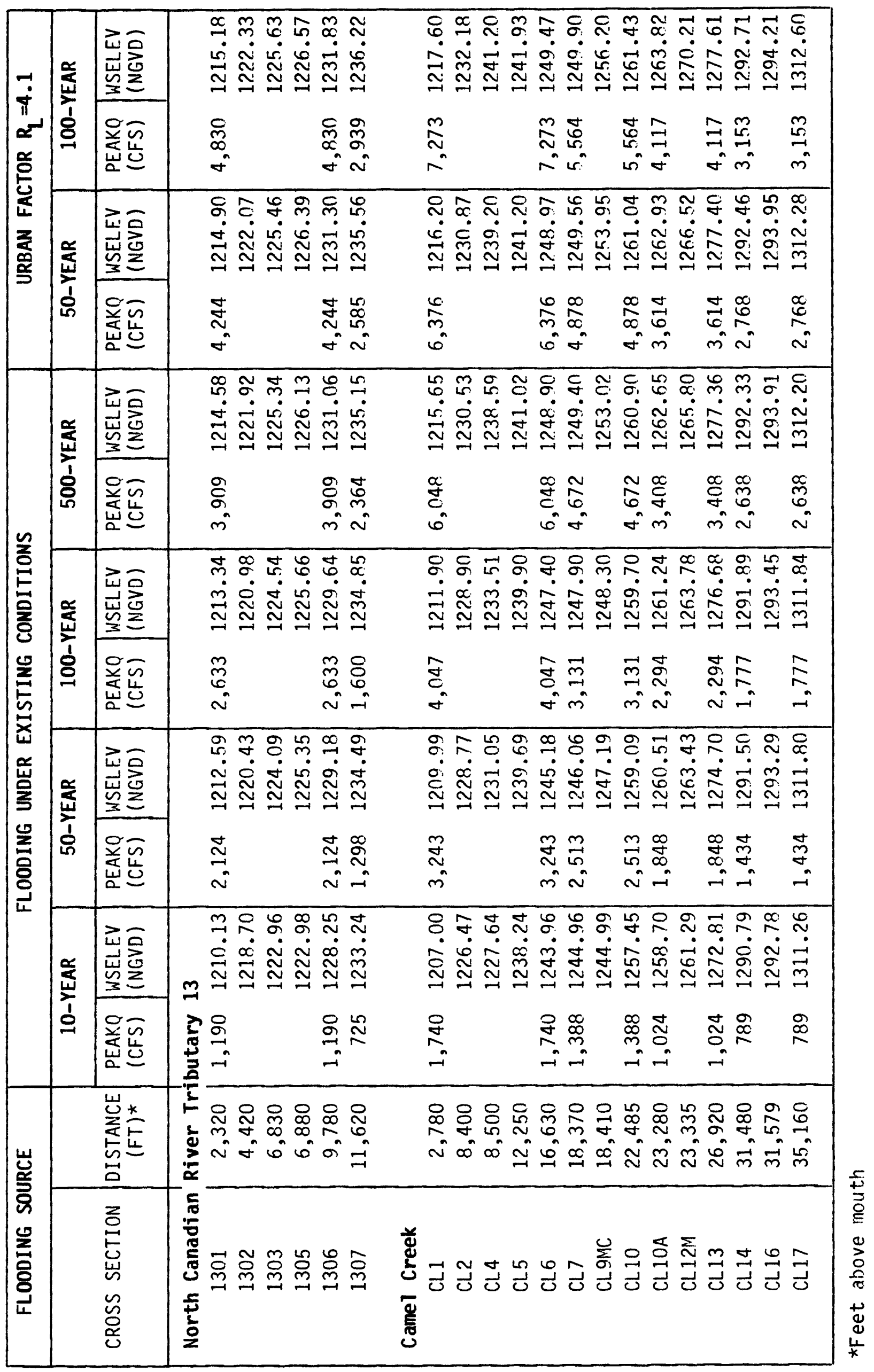




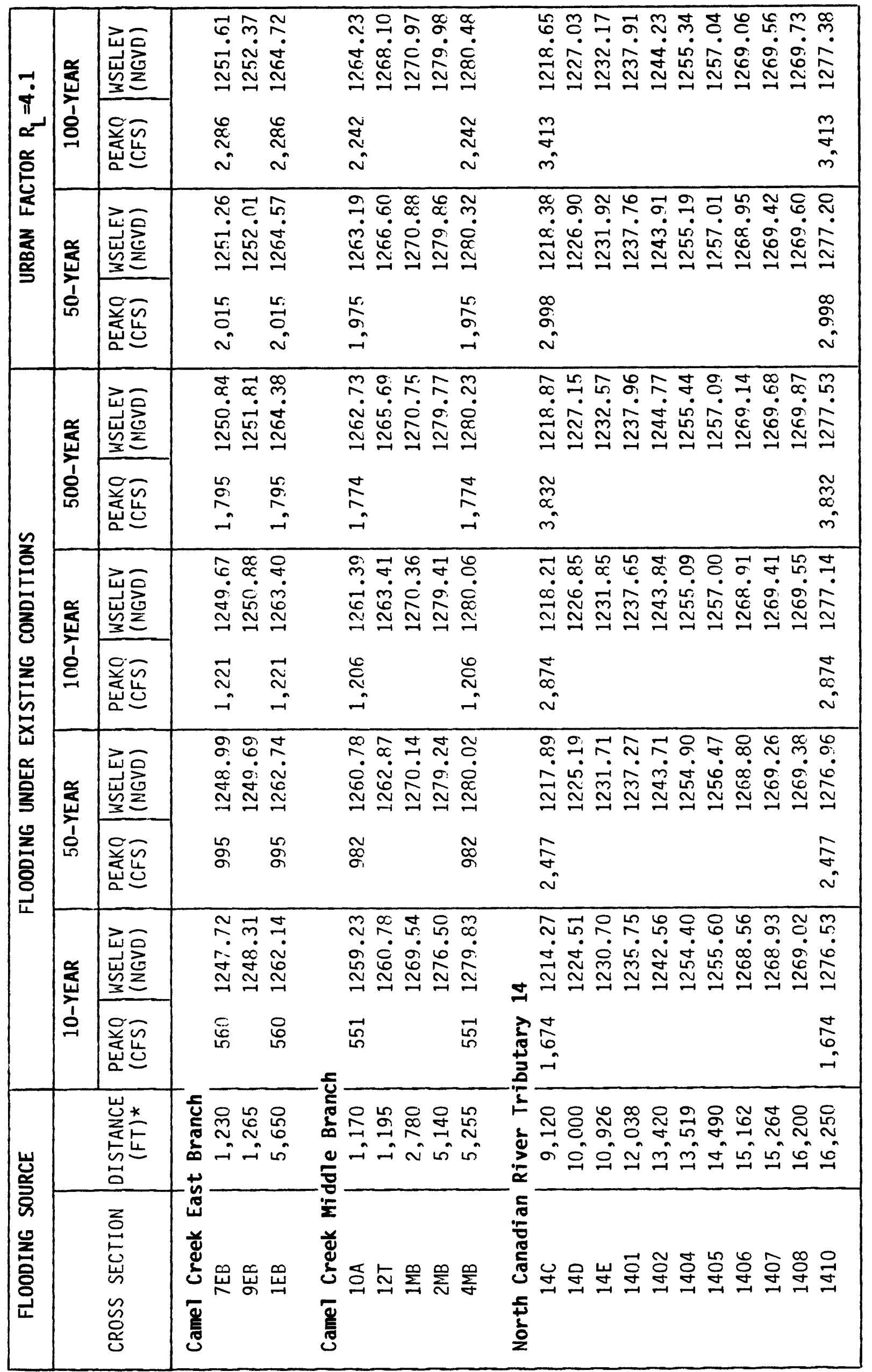




\begin{tabular}{|c|c|c|c|c|c|c|}
\hline \multirow{3}{*}{ 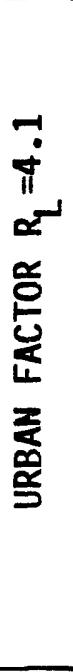 } & 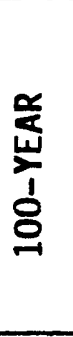 & 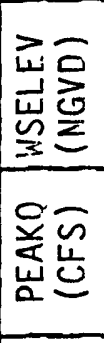 & 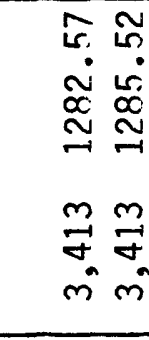 & \multicolumn{2}{|l|}{ 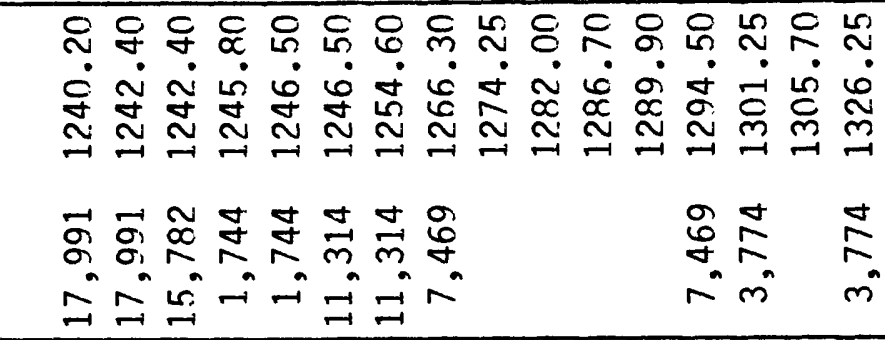 } & 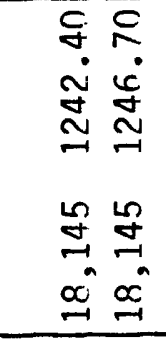 \\
\hline & 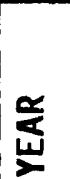 & 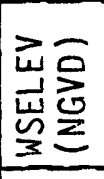 & 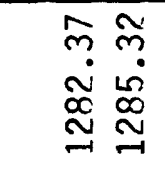 & 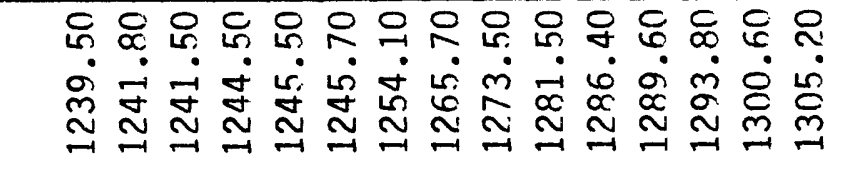 & $\begin{array}{l}\stackrel{c}{\vdots} \\
\stackrel{0}{\sim} \\
\stackrel{m}{r}\end{array}$ & 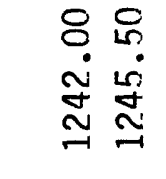 \\
\hline & is & 恙怘 & $\begin{array}{l}\infty \\
\sigma \\
\sigma \\
\sigma \\
\sim \\
\sim \\
\sim\end{array}$ & 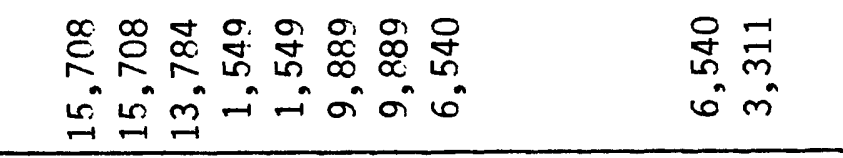 & $\begin{array}{l}\overrightarrow{7} \\
m \\
m\end{array}$ & 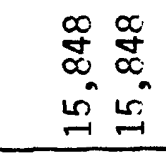 \\
\hline \multirow{8}{*}{ 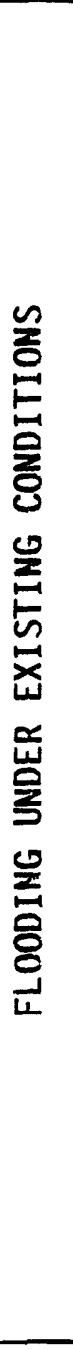 } & \multirow{2}{*}{ 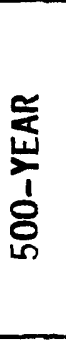 } & 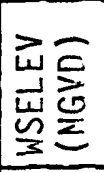 & 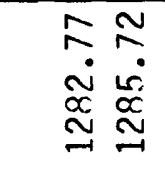 & 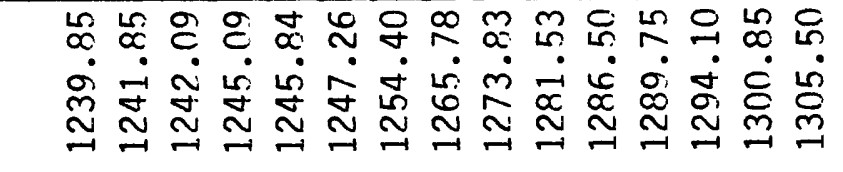 & $\stackrel{f}{\stackrel{\check{c}}{m}}$ & 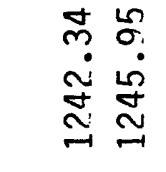 \\
\hline & & 尼志 & $\begin{array}{l}\tilde{m} \underset{m}{\tilde{m}} \\
\infty \\
\tilde{m}\end{array}$ & 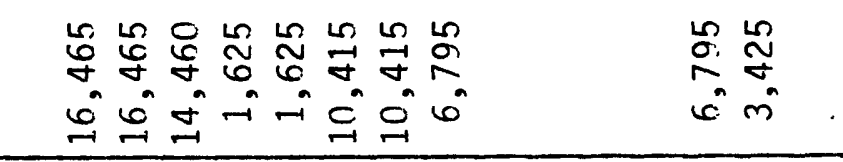 & 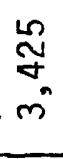 & 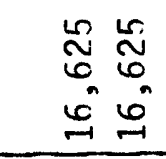 \\
\hline & \multirow{2}{*}{ 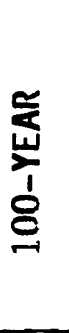 } & 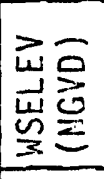 & 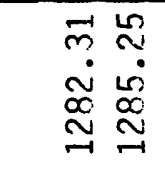 & 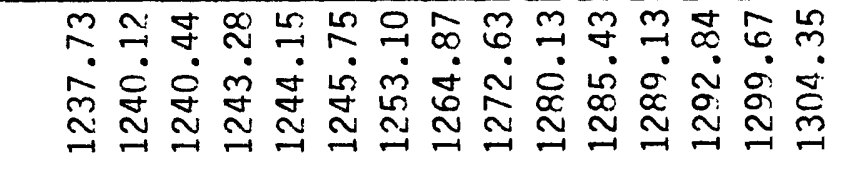 & $\begin{array}{l}m \\
\infty \\
\dot{\sim} \\
\tilde{m}\end{array}$ & 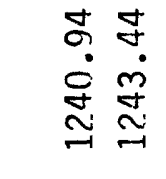 \\
\hline & & 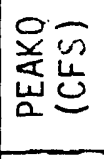 & $\begin{array}{l}\sim \\
\infty \\
\infty \\
\sim \\
\sim\end{array}$ & 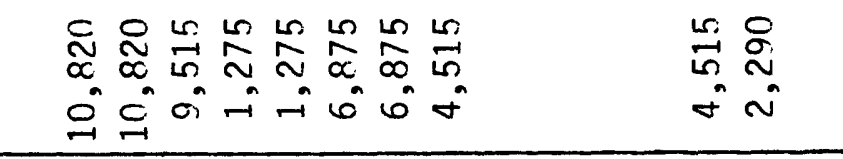 & I & 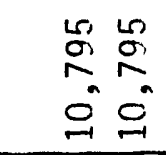 \\
\hline & \multirow{2}{*}{ 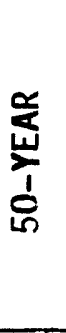 } & 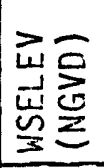 & 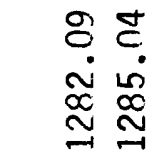 & 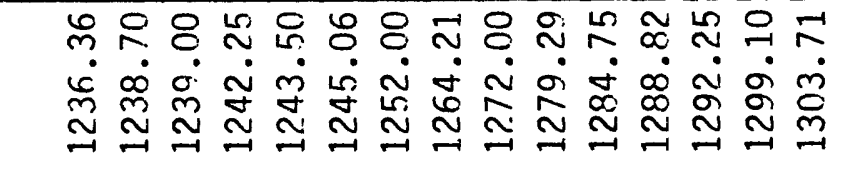 & 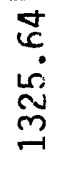 & 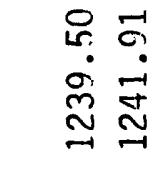 \\
\hline & & 运荝 & $\begin{array}{r}\tilde{\sigma} \hat{\sigma} \\
-\tilde{\sigma}\end{array}$ & 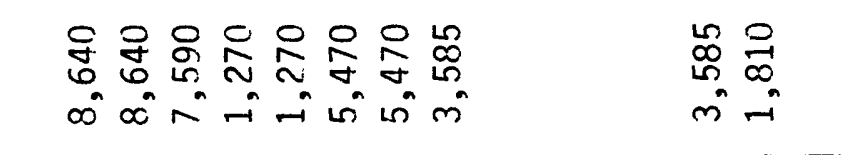 & $\begin{array}{l}0 \\
\infty \\
-1\end{array}$ & 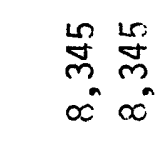 \\
\hline & \multirow{2}{*}{ 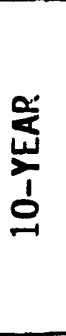 } & 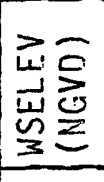 & 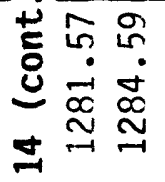 & 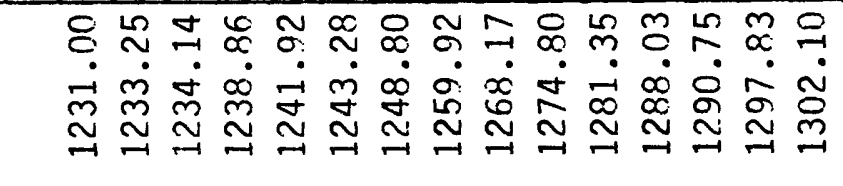 & $\stackrel{m}{\longrightarrow}$ & 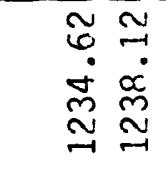 \\
\hline & & 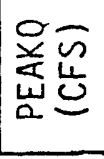 & 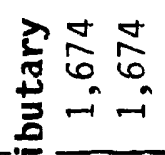 & 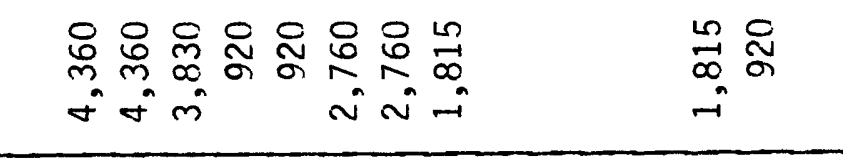 & న్ & $\begin{array}{r}0 \\
\infty \\
\sigma \\
\sigma \\
-m ~ \\
-m\end{array}$ \\
\hline \multirow{2}{*}{ 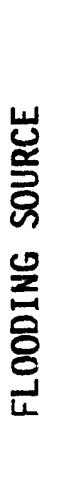 } & & 岕芯 & 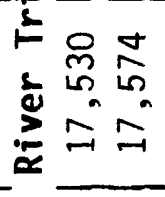 & 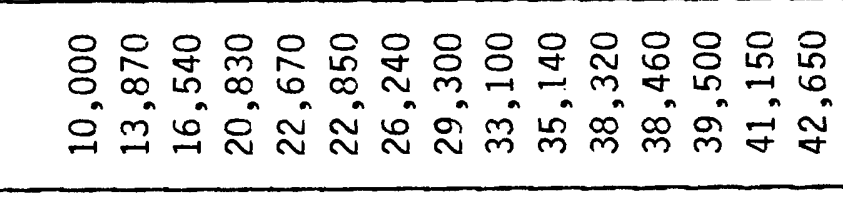 & $\stackrel{8}{8}$ & 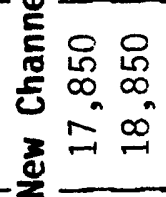 \\
\hline & & 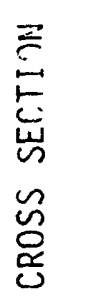 & 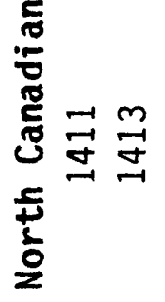 & 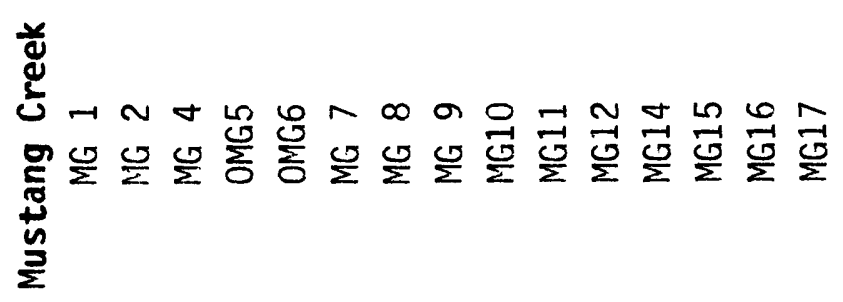 & $\frac{9}{2}$ & 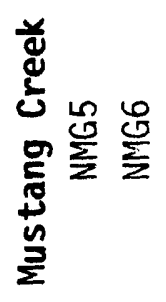 \\
\hline
\end{tabular}




\begin{tabular}{|c|c|c|c|c|c|c|c|c|}
\hline \multirow{4}{*}{ 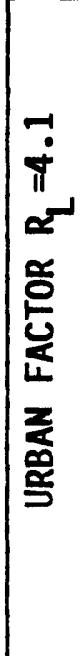 } & \multirow{2}{*}{$\mid \begin{array}{c}\frac{0}{4} \\
\frac{1}{1} \\
0 \\
0\end{array}$} & 崖㤩 & 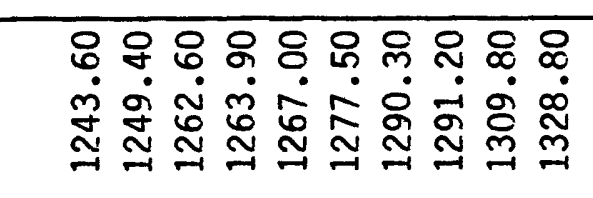 & 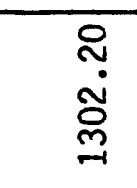 & \multicolumn{4}{|c|}{ 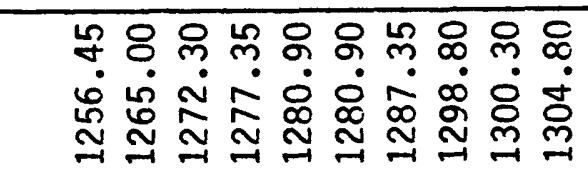 } \\
\hline & & 送出 & 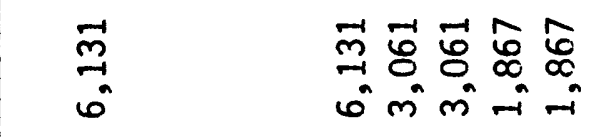 & $\begin{array}{l}\stackrel{0}{a} \\
\infty \\
\sim\end{array}$ & $\begin{array}{l}\frac{0}{\sqrt{n}} \\
\infty\end{array}$ & $\begin{array}{ll}\stackrel{0}{*} & \overrightarrow{1} \\
0 & 0 \\
\infty & 0\end{array}$ & 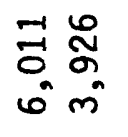 & $\begin{array}{l}\mathscr{N} \\
\tilde{\sigma} \\
m\end{array}$ \\
\hline & \multirow[t]{2}{*}{ 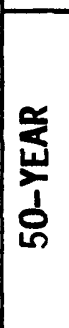 } & 崖念 & 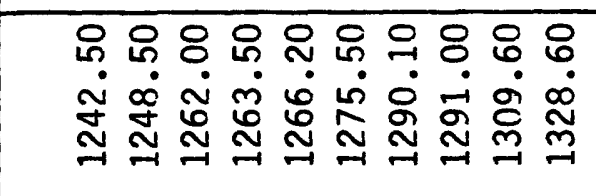 & 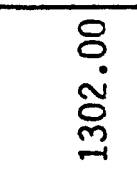 & \multicolumn{4}{|c|}{ 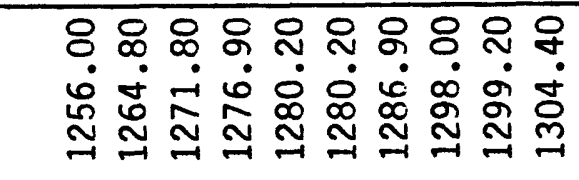 } \\
\hline & & 递苍 & 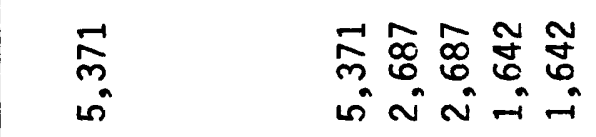 & $\begin{array}{l}\hat{o} \\
\mathfrak{i}\end{array}$ & $\begin{array}{l}\mathbb{Q} \\
\infty \\
0\end{array}$ & 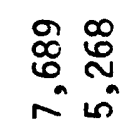 & 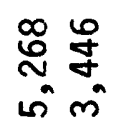 & $\begin{array}{l}\mathscr{O} \\
\dot{q} \\
m\end{array}$ \\
\hline \multirow{8}{*}{ 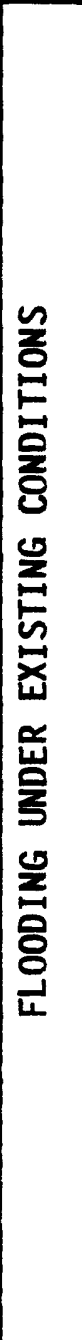 } & \multirow{2}{*}{ 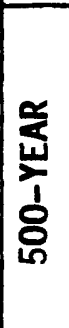 } & 岕量 & 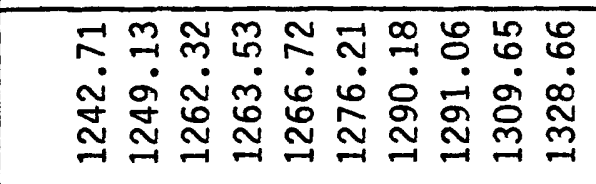 & 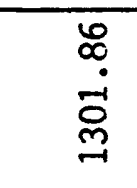 & \multicolumn{4}{|c|}{ 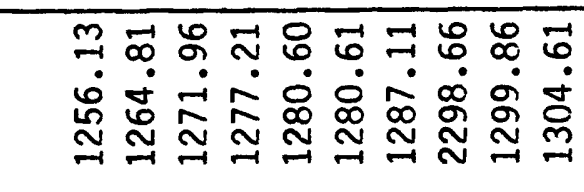 } \\
\hline & & 造怠 & 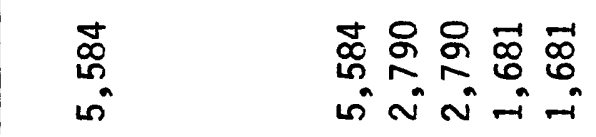 & 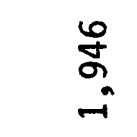 & $\underset{\infty}{\sim}$ & 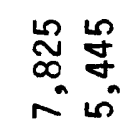 & 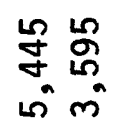 & 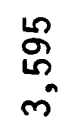 \\
\hline & \multirow{2}{*}{ 愛 } & 㞾高 & 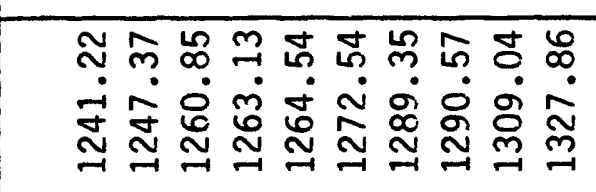 & in & \multicolumn{4}{|c|}{ 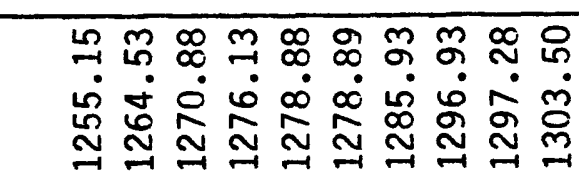 } \\
\hline & & 递点 & 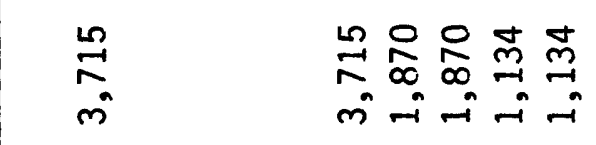 & $\vec{F}$ & "م & 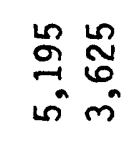 & 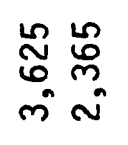 & in \\
\hline & \multirow{2}{*}{ 茓 } & 崖㤩 & 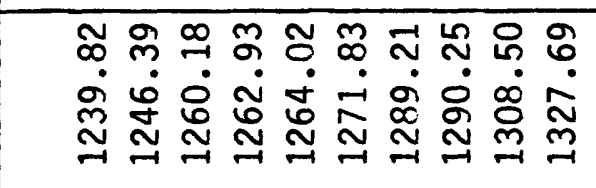 & $\begin{array}{l}\vec{i} \\
\dot{\vec{I}} \\
\rightarrow\end{array}$ & \multicolumn{4}{|c|}{ 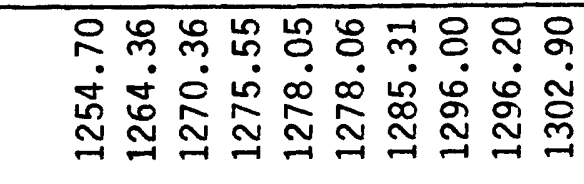 } \\
\hline & & 递出 & 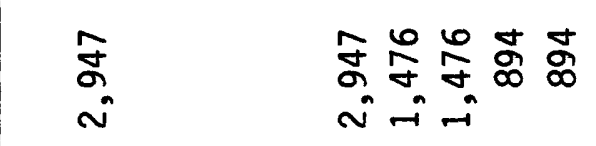 & $\stackrel{0}{\stackrel{0}{-}}$ & $\stackrel{L}{\exists}$ & 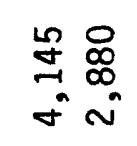 & $\begin{array}{ll}\infty & \infty \\
\infty & \infty \\
\infty & \infty \\
N & -1\end{array}$ & $\stackrel{+\infty}{\infty}$ \\
\hline & \multirow{2}{*}{$\underset{\frac{\alpha}{5}}{\frac{\alpha}{5}}$} & 崖㤩 & 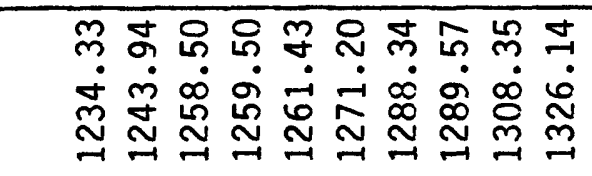 & 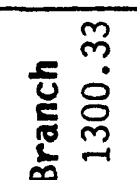 & \multicolumn{4}{|c|}{ 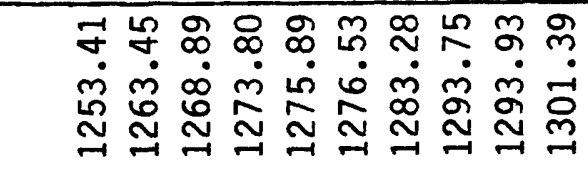 } \\
\hline & & 产岕 & 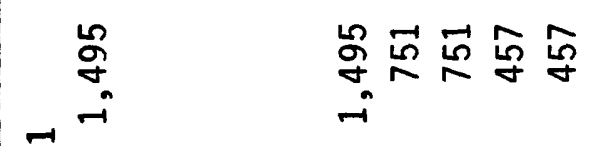 & 芜 \% & & 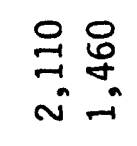 & 畩总 & 占 \\
\hline \multirow{2}{*}{ 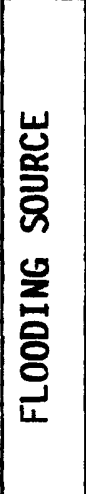 } & & 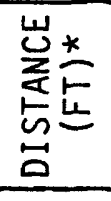 & 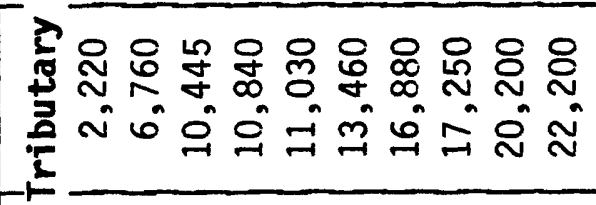 & 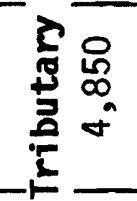 & \multicolumn{4}{|c|}{ 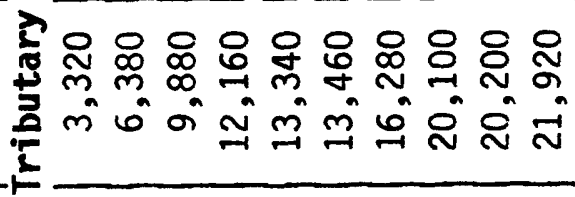 } \\
\hline & & 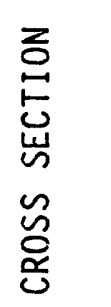 & 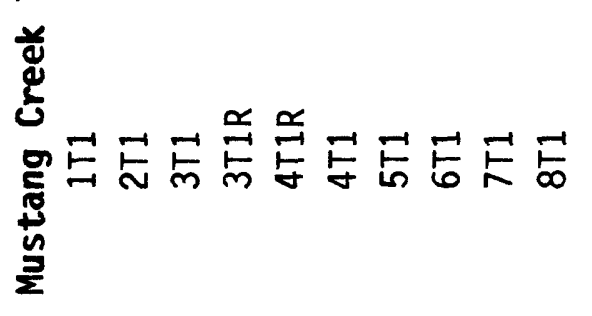 & 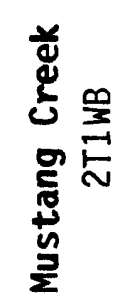 & \multicolumn{4}{|l|}{ 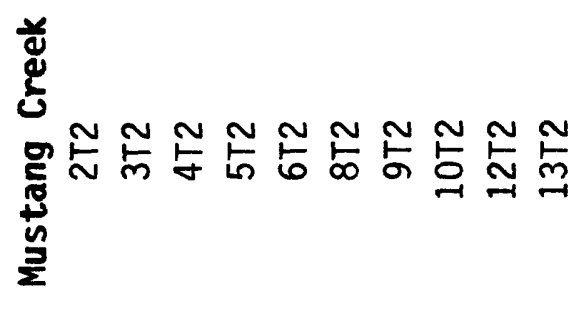 } \\
\hline
\end{tabular}




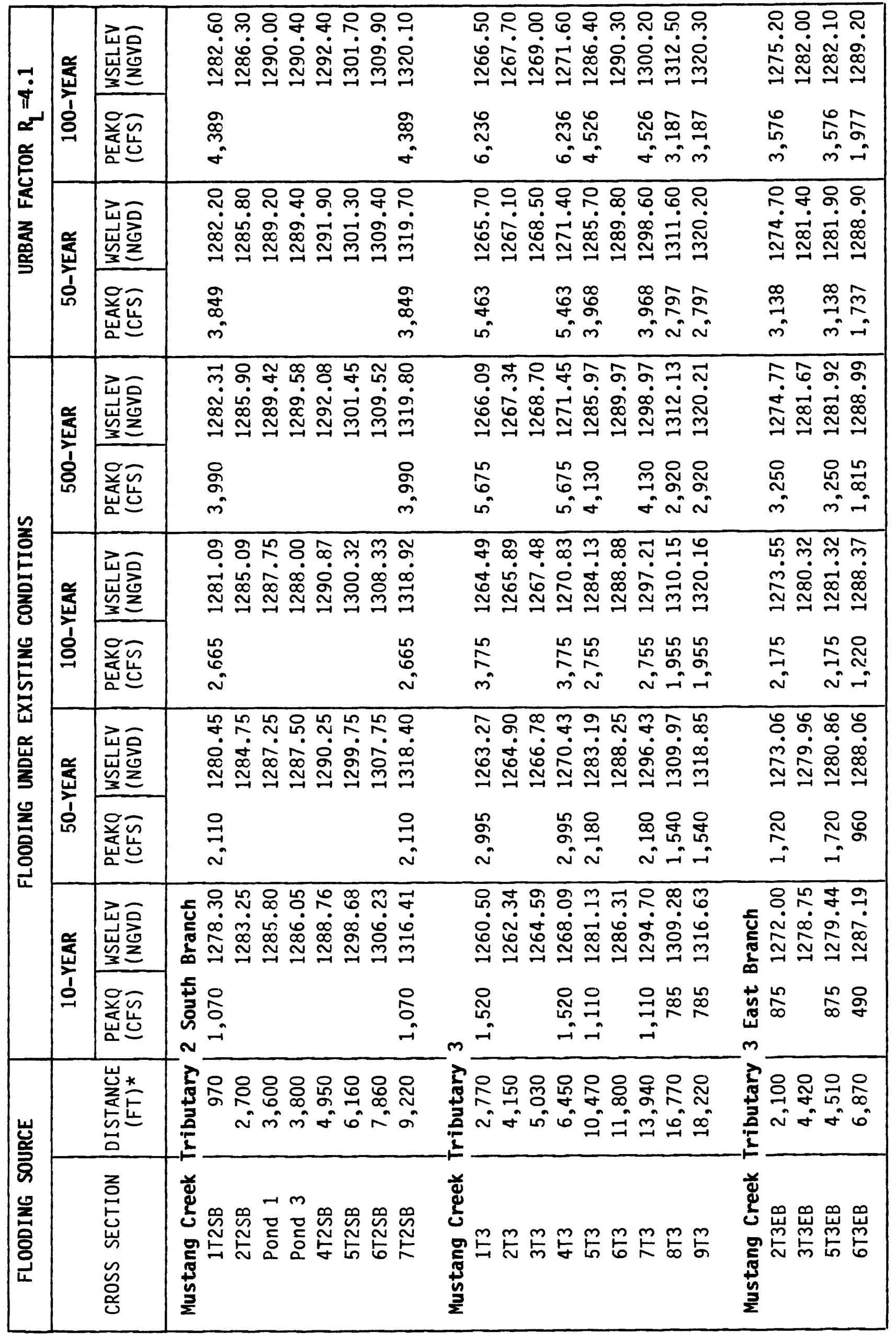




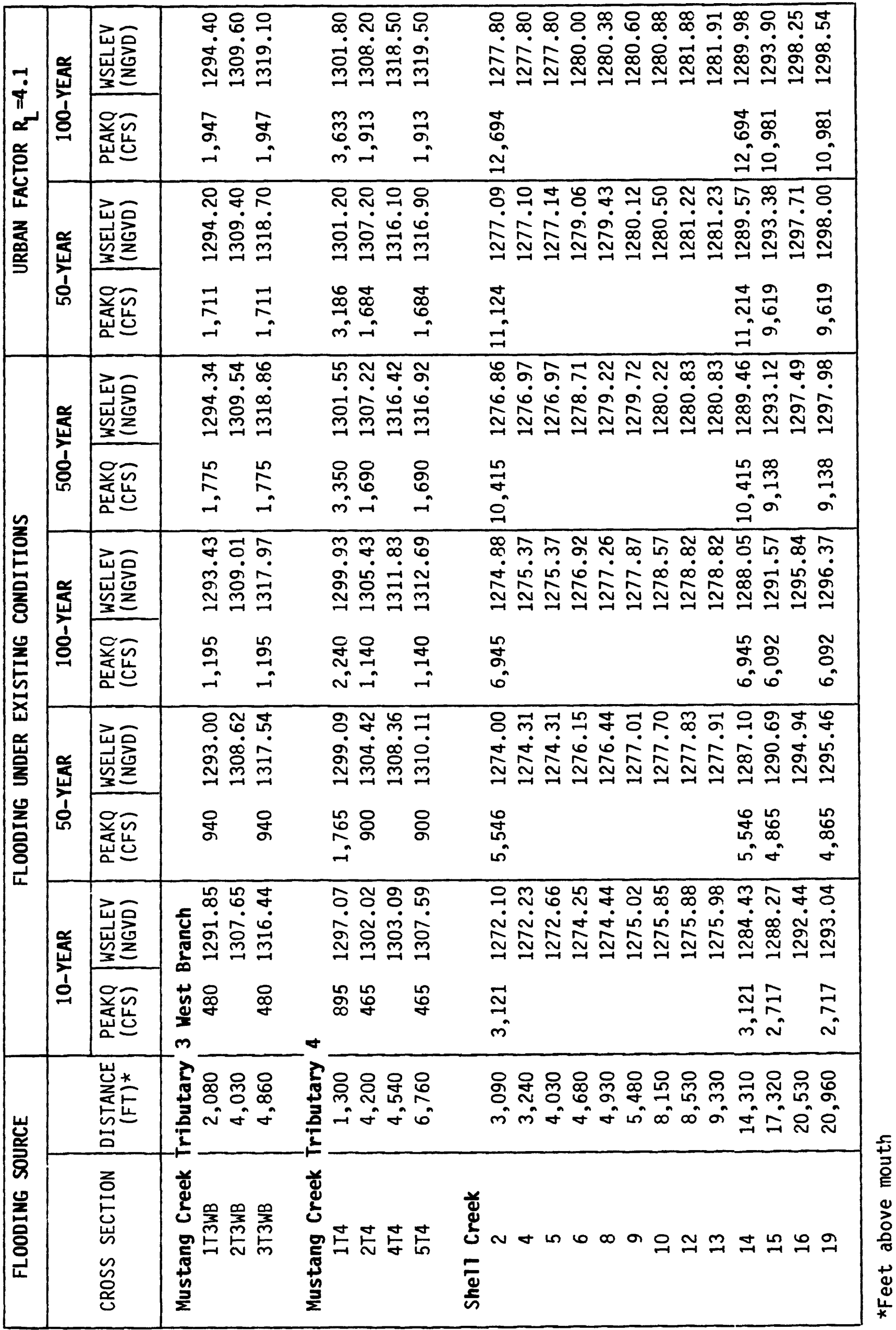




\begin{tabular}{|c|c|c|c|c|c|c|c|}
\hline \multirow{4}{*}{ 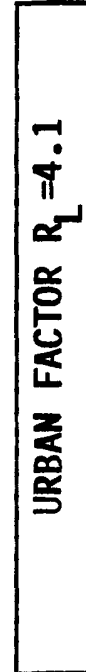 } & \multirow{2}{*}{$\mid \frac{2}{4}$} & \multirow{2}{*}{ 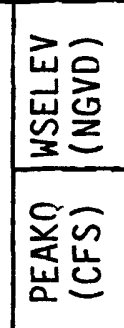 } & \multicolumn{3}{|c|}{ 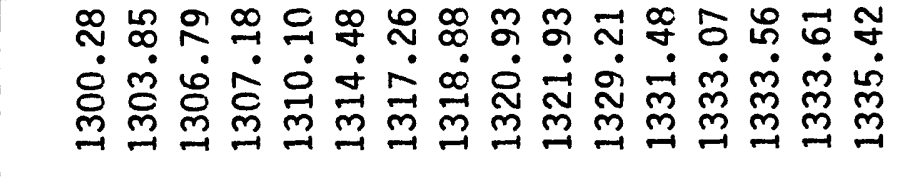 } & \multicolumn{2}{|c|}{ 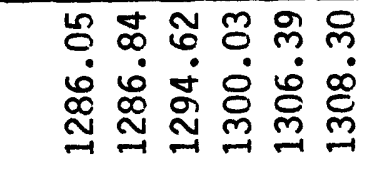 } \\
\hline & & & añ & 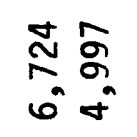 & 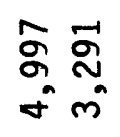 & 号 & $\begin{array}{l}\text { 용 } \\
\text { N }\end{array}$ \\
\hline & \multirow{2}{*}{ 总 } & 岀高 & \multicolumn{3}{|c|}{ 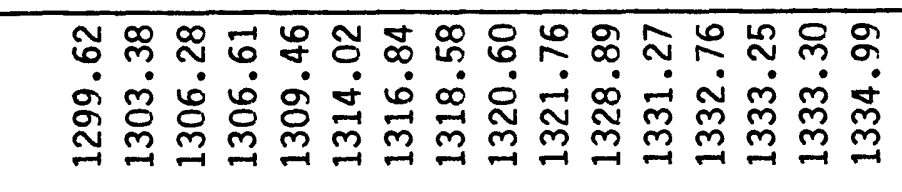 } & \multicolumn{2}{|c|}{ 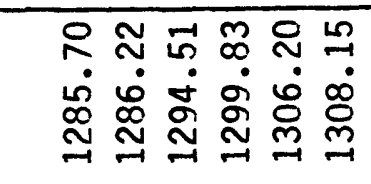 } \\
\hline & & 递念 & $\begin{array}{l}8 \infty \\
\stackrel{0}{0} \\
\infty \\
\infty \\
\infty\end{array}$ & $\begin{array}{l}\infty \\
\infty \\
\infty \\
\infty \\
\omega_{n}^{\infty} \\
\infty\end{array}$ & 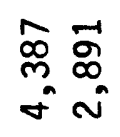 & 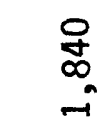 & $\underset{\substack{0 \\
-1}}{-1}$ \\
\hline \multirow{8}{*}{ 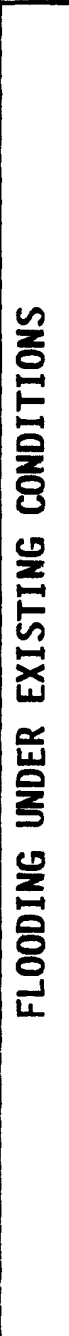 } & \multirow{2}{*}{ 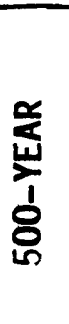 } & 岂高 & \multicolumn{3}{|c|}{ 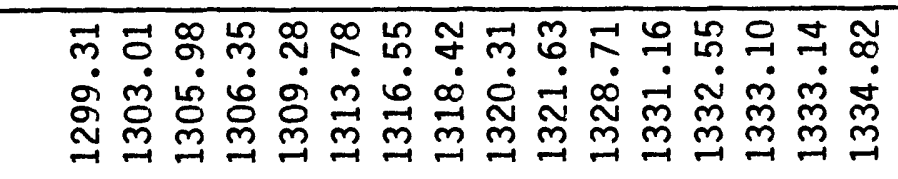 } & \multicolumn{2}{|c|}{ 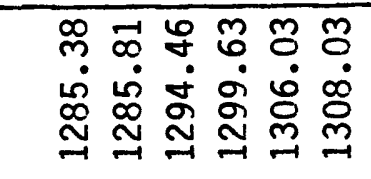 } \\
\hline & & 窇售 & 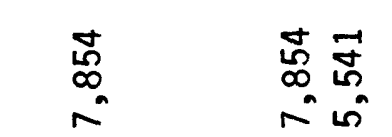 & 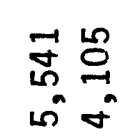 & 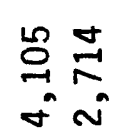 & $\underset{\substack{\infty \\
0 \\
\hdashline}}{-1}$ & $\begin{array}{l}\mathfrak{m} \\
\infty \\
0 \\
-1\end{array}$ \\
\hline & \multirow{2}{*}{$\begin{array}{l}\frac{\alpha}{5} \\
\frac{1}{1} \\
0 \\
0\end{array}$} & 崖 & \multicolumn{3}{|c|}{ 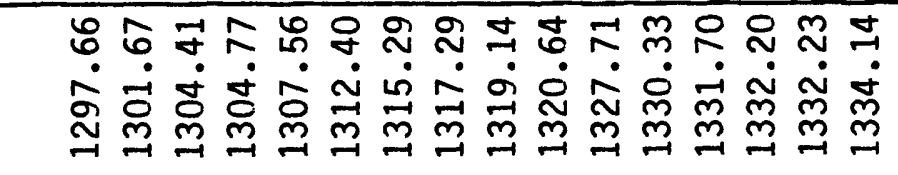 } & \multicolumn{2}{|c|}{ 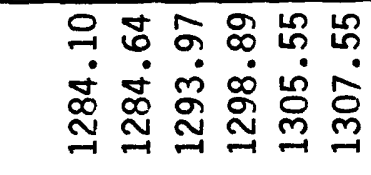 } \\
\hline & & 送出 & 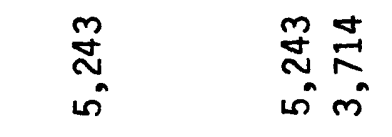 & 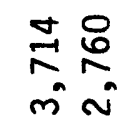 & $\begin{array}{l}8 \vec{m} \\
\stackrel{0}{\infty} \\
\vec{n}\end{array}$ & $\underset{-}{\stackrel{m}{G}}$ & $\underset{\underset{+}{+}}{\stackrel{m}{-1}}$ \\
\hline & \multirow{2}{*}{ 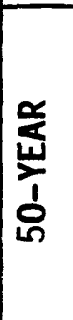 } & 岂空 & \multicolumn{3}{|c|}{ 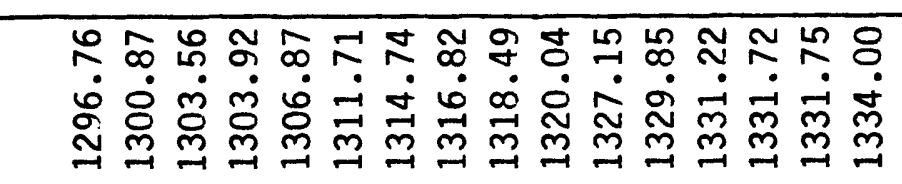 } & \multicolumn{2}{|c|}{ 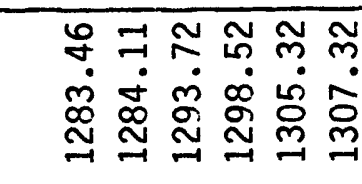 } \\
\hline & & 递出 & 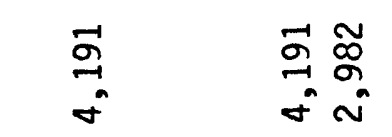 & $\begin{array}{l}\tilde{\infty} \text { N } \\
\text { N } \\
\sim\end{array}$ & $\begin{array}{l}\tilde{N} \\
\stackrel{a}{f} \\
\sim\end{array}$ & ริ & నิ \\
\hline & \multirow{2}{*}{ 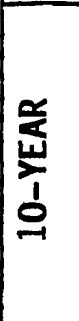 } & 崖 & \multicolumn{3}{|c|}{ 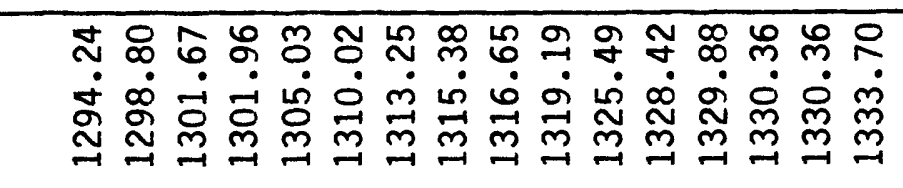 } & \multicolumn{2}{|c|}{ 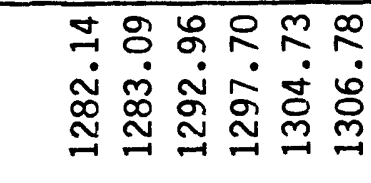 } \\
\hline & & 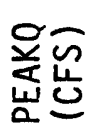 & 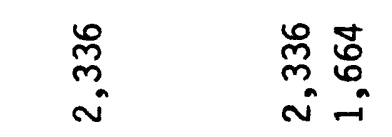 & 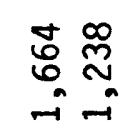 & $\underset{\sim}{\stackrel{\infty}{\sim}} \stackrel{0}{\infty}$ & $\stackrel{\infty}{\vec{H}}$ & $\stackrel{\infty}{n}$ \\
\hline \multirow{2}{*}{ 容 } & & 岕芯 & \multicolumn{3}{|c|}{ 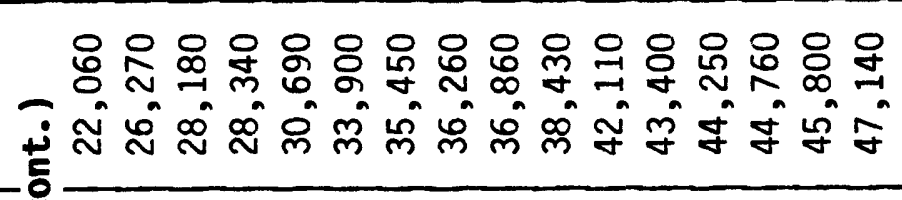 } & \multicolumn{2}{|c|}{ 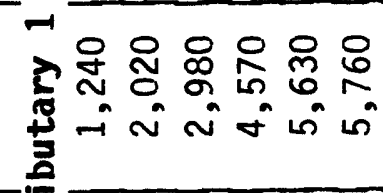 } \\
\hline & & 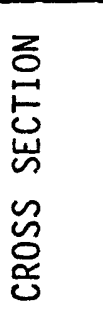 & \multicolumn{3}{|c|}{$\begin{array}{l}\bar{\Psi} \\
\bar{d} \\
\bar{\Phi} \\
\bar{\Sigma}\end{array}$} & \multicolumn{2}{|c|}{ 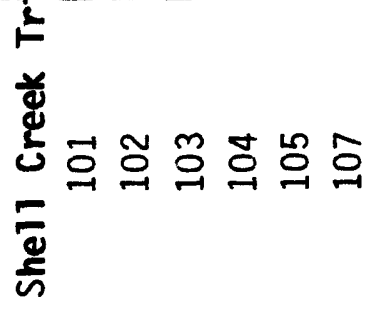 } \\
\hline
\end{tabular}




\begin{tabular}{|c|c|c|c|c|c|c|c|}
\hline \multirow{4}{*}{ 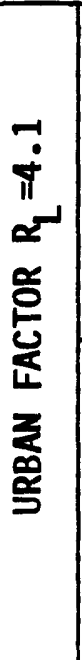 } & \multirow{2}{*}{ 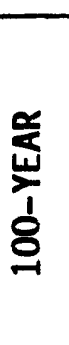 } & 峞高 & 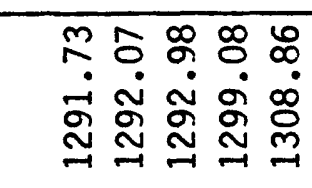 & \multicolumn{3}{|c|}{ 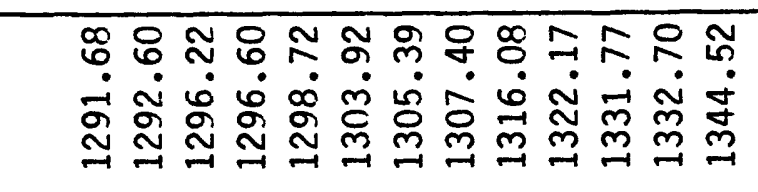 } & \multirow{2}{*}{ 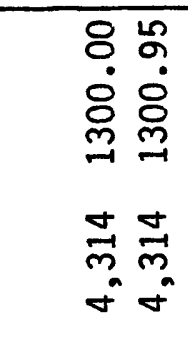 } \\
\hline & & 恙出 & $\stackrel{\infty}{n}_{n}^{\infty}$ & & 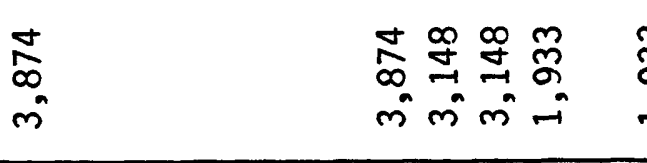 & $\stackrel{m}{\tilde{g}}_{\tilde{r}}$ & \\
\hline & 空 & 岂这 & 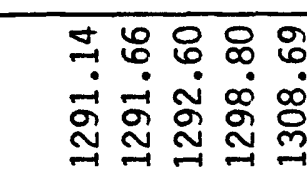 & & 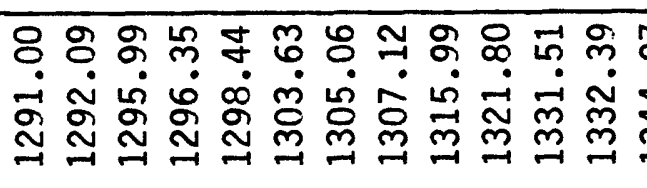 & 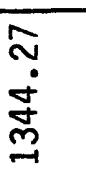 & 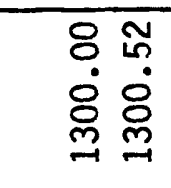 \\
\hline & d & 道岂 & $\begin{array}{ll}\stackrel{2}{0} & \stackrel{2}{N} \\
\sim & N\end{array}$ & & 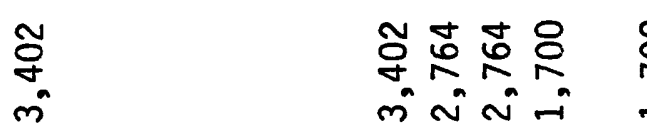 & $\underset{i}{\stackrel{9}{\circ}}$ & $\begin{array}{l}\infty \\
\stackrel{\infty}{\infty} \\
\stackrel{\infty}{m} \\
\dot{m} \\
m\end{array}$ \\
\hline \multirow{8}{*}{ 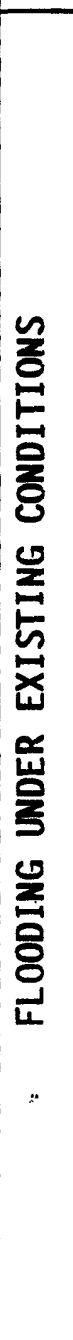 } & \multirow{2}{*}{ 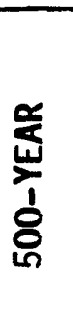 } & 峞是 & 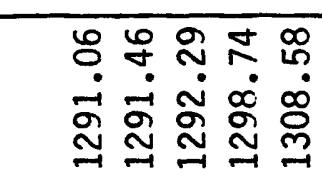 & \multicolumn{3}{|c|}{ 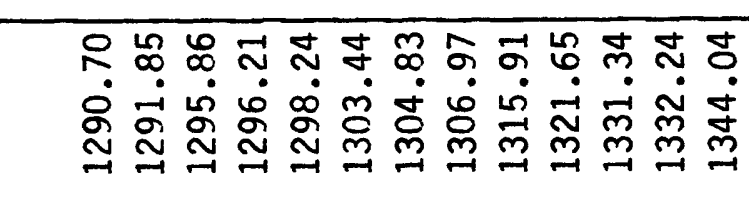 } & 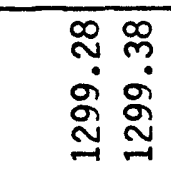 \\
\hline & & 道允 & $\begin{array}{ll}\stackrel{g}{m} & \stackrel{m}{7} \\
\sim & \sim\end{array}$ & & 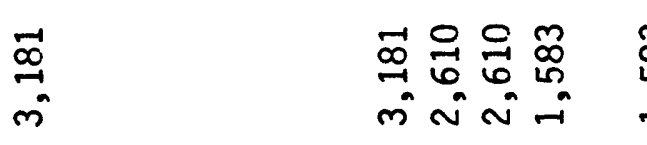 & 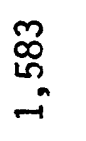 & 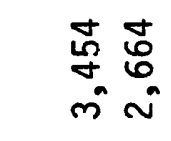 \\
\hline & \multirow{2}{*}{ 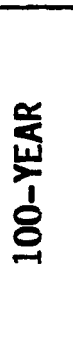 } & 山气 & 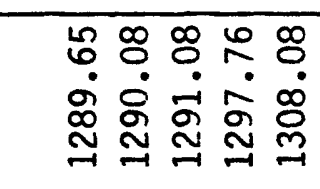 & \multicolumn{3}{|c|}{ 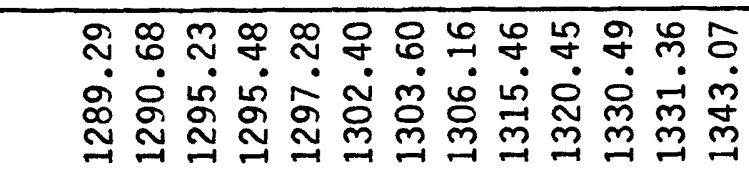 } & 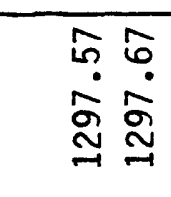 \\
\hline & & 递芯 & $\underset{f}{\mathbb{L}}$ & & 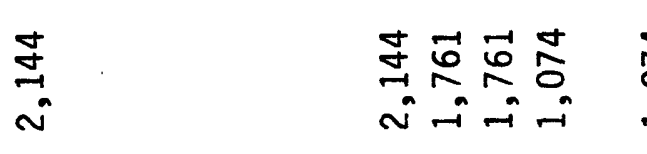 & $\underset{i}{i}$ & 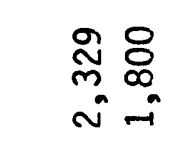 \\
\hline & \multirow{2}{*}{$\begin{array}{l}\frac{\infty}{5} \\
\frac{1}{1} \\
\frac{1}{0}\end{array}$} & 岀㝵 & 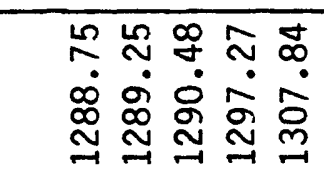 & \multicolumn{3}{|c|}{ 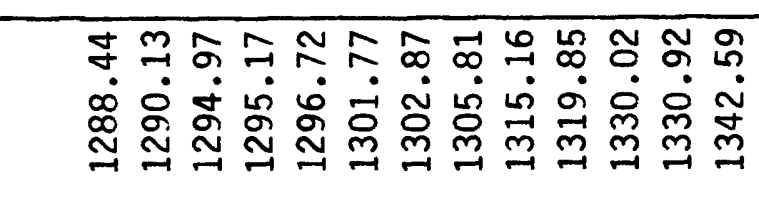 } & 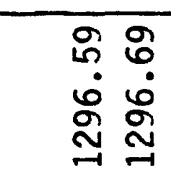 \\
\hline & & 递苍 & $\underset{8}{0} \stackrel{8}{-1}$ & & 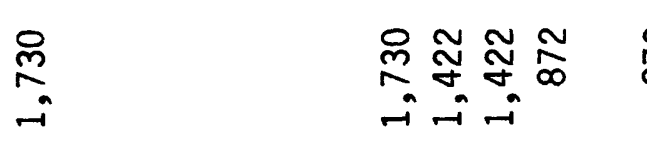 & $\underset{\infty}{N}$ & $\begin{array}{l}-15 \\
\infty \\
\infty \\
-i=-1\end{array}$ \\
\hline & \multirow{2}{*}{$\begin{array}{c}\frac{x}{4} \\
\frac{1}{1} \\
0\end{array}$} & 岂竞 & 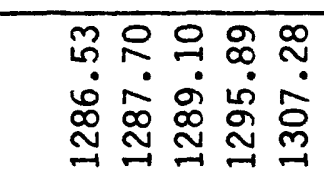 & & 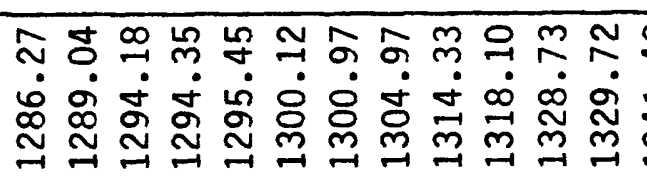 & 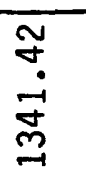 & 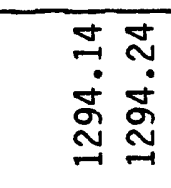 \\
\hline & & 这出 & 焉 & & 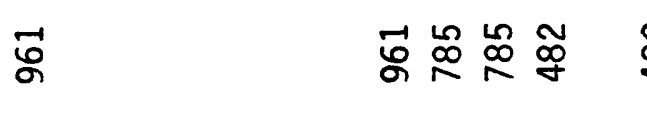 & $\stackrel{\sim}{\infty}$ & $\stackrel{m}{\stackrel{m}{0}} \underset{-i}{m}$ \\
\hline \multirow{2}{*}{ 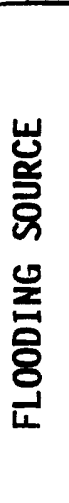 } & & 岁岕 & 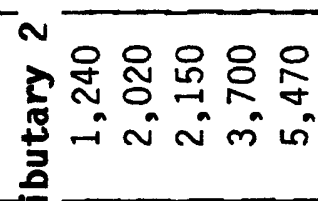 & \multicolumn{3}{|c|}{ 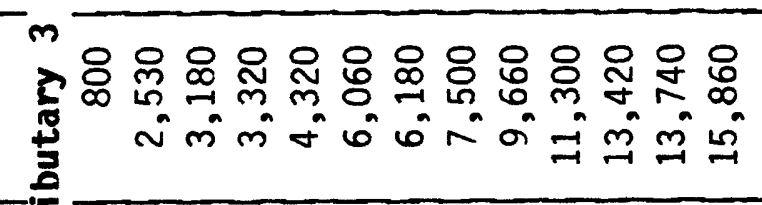 } & 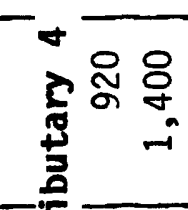 \\
\hline & & 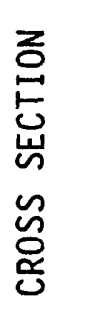 & 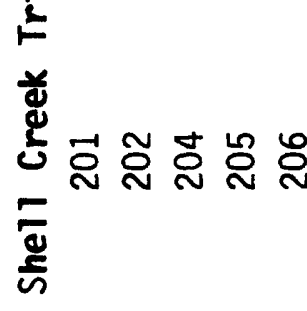 & 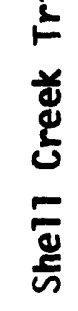 & 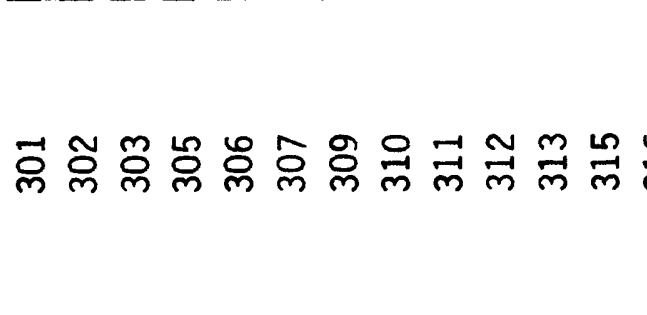 & & 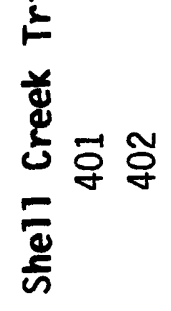 \\
\hline
\end{tabular}




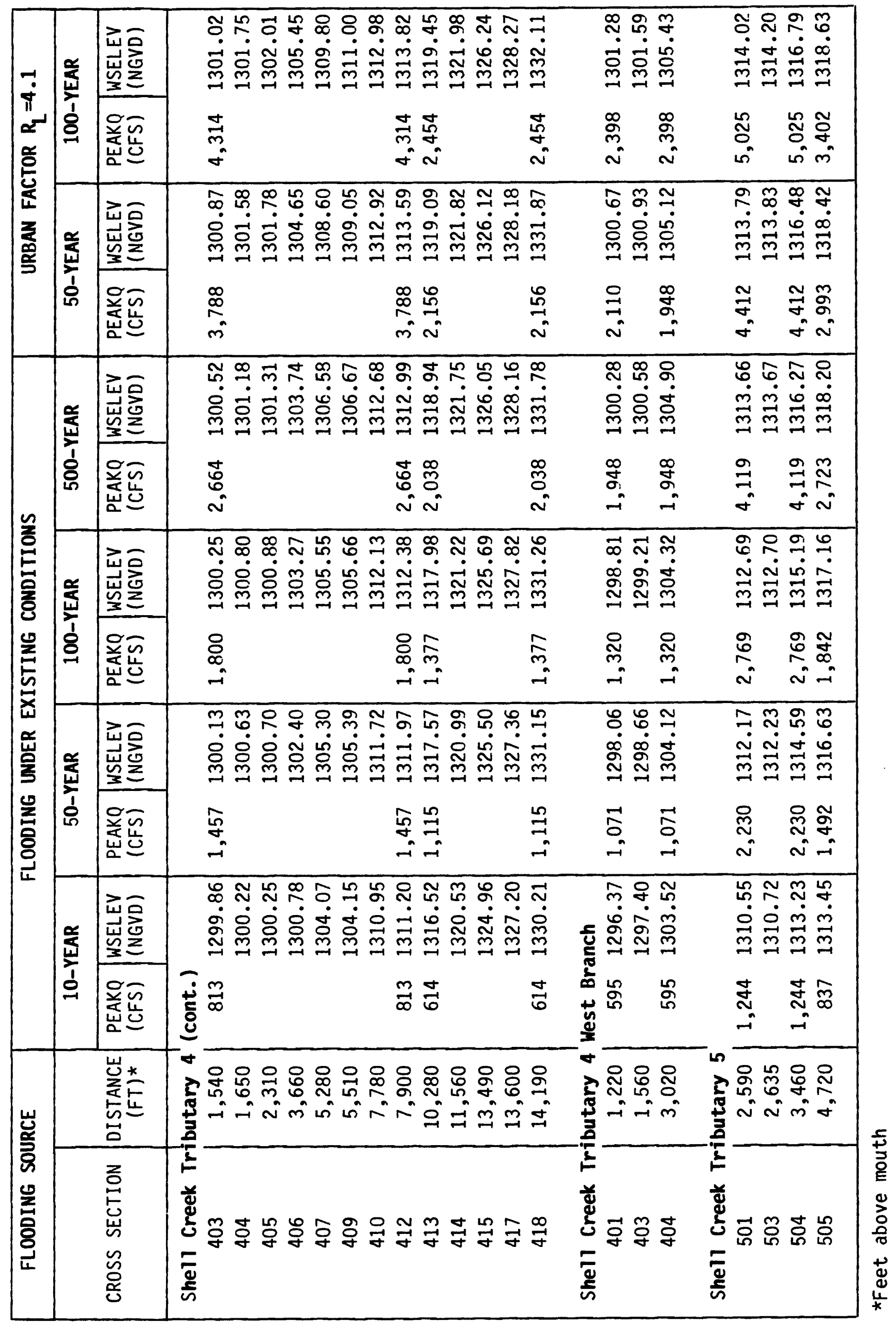




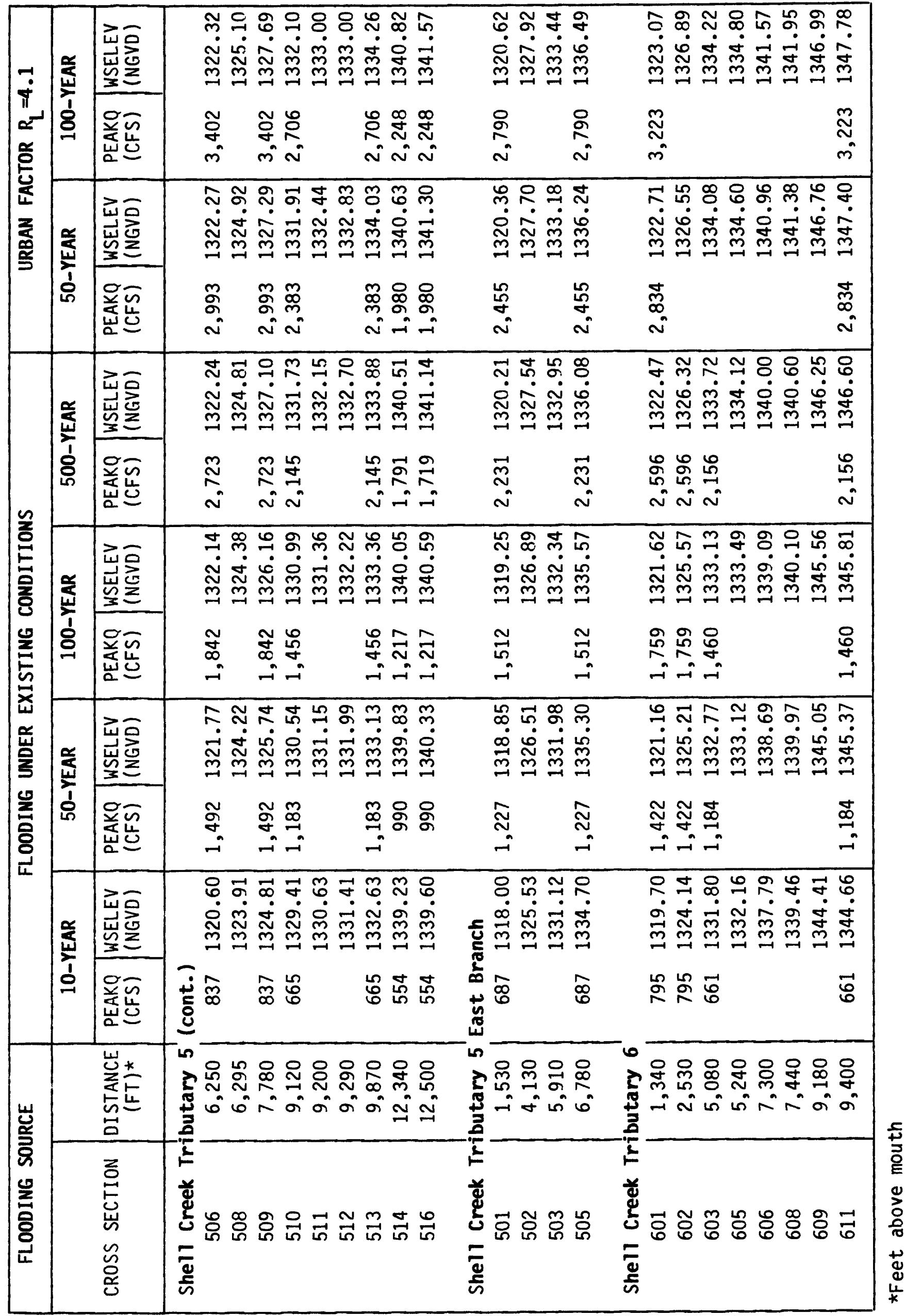




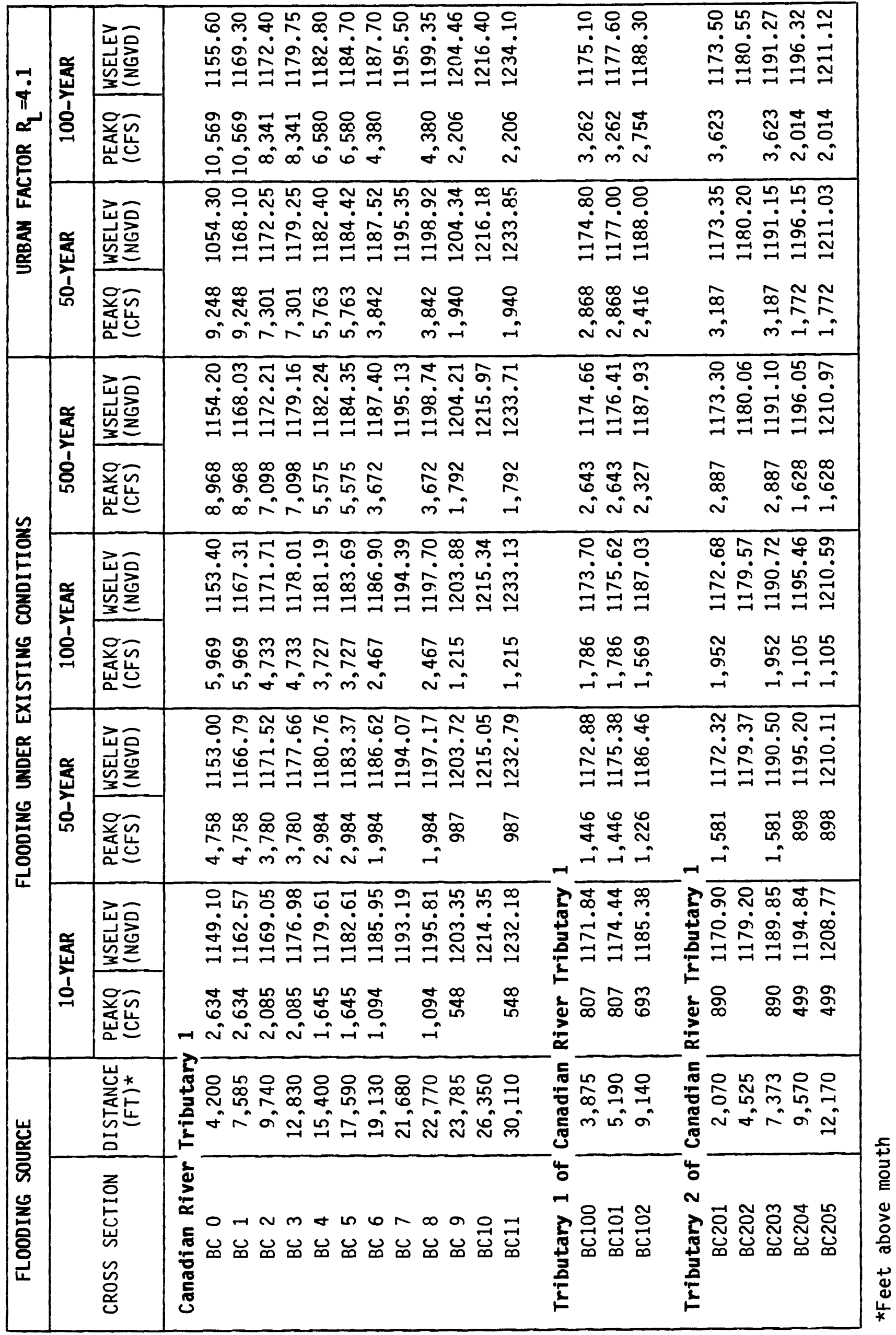




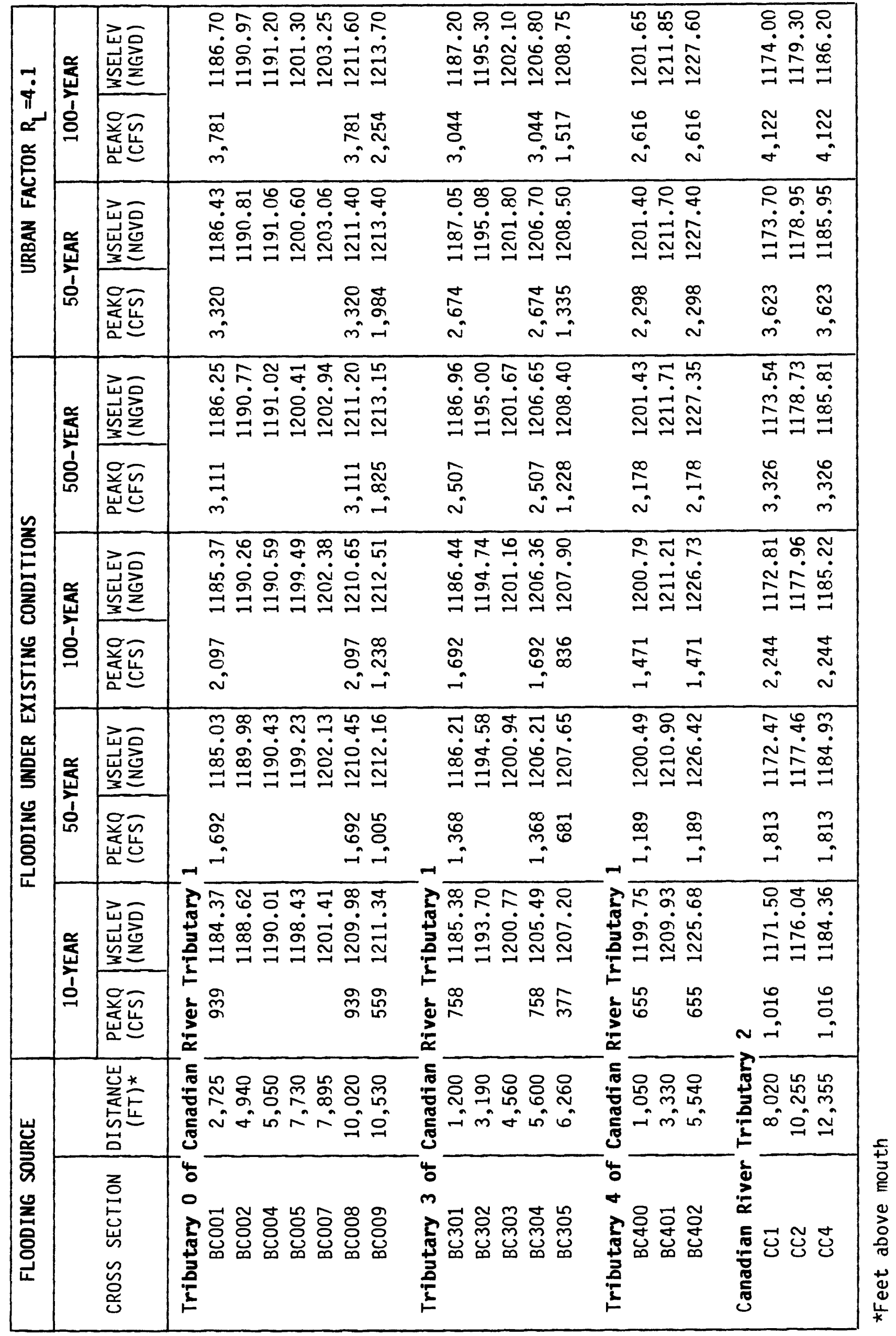




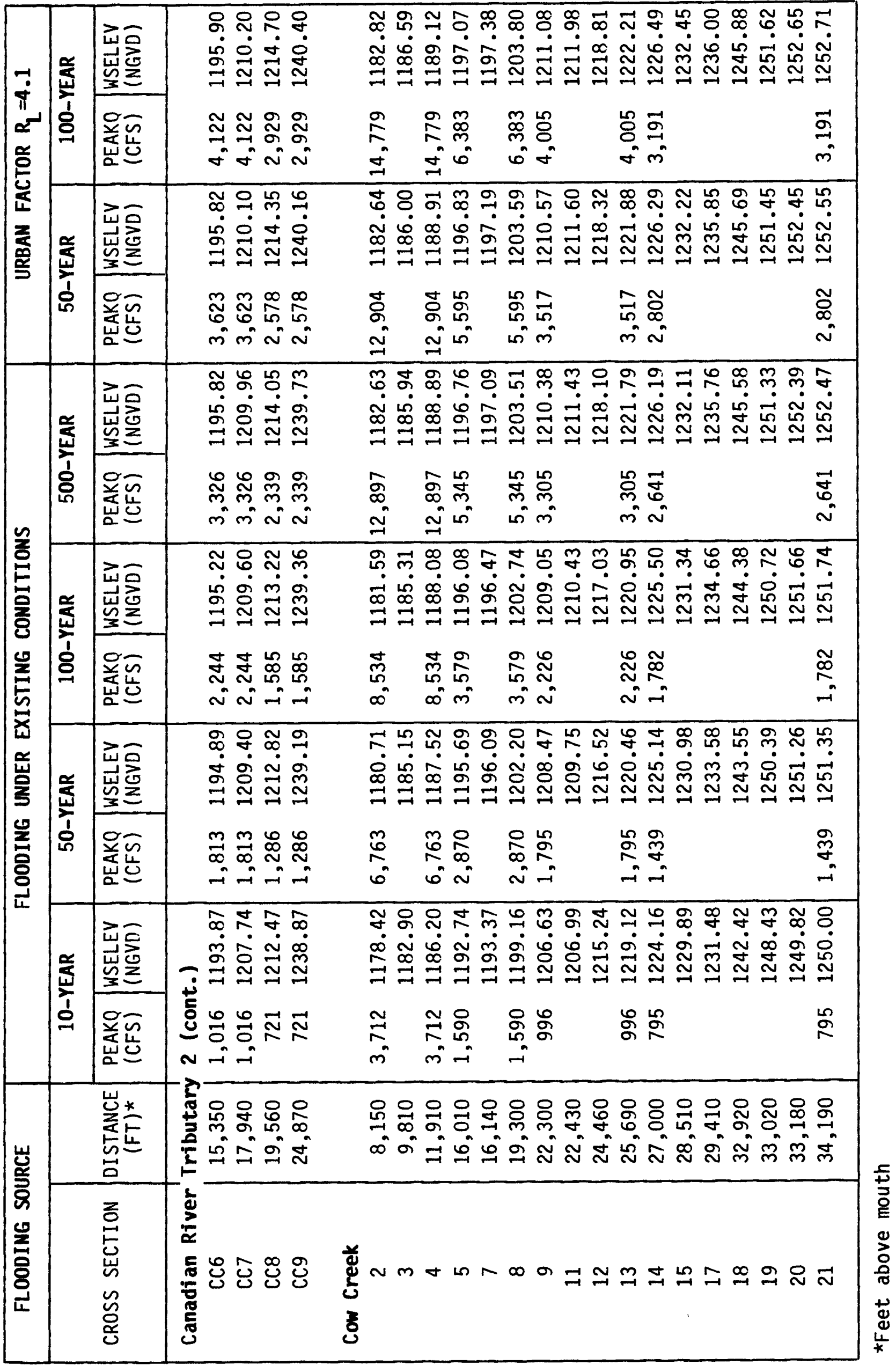




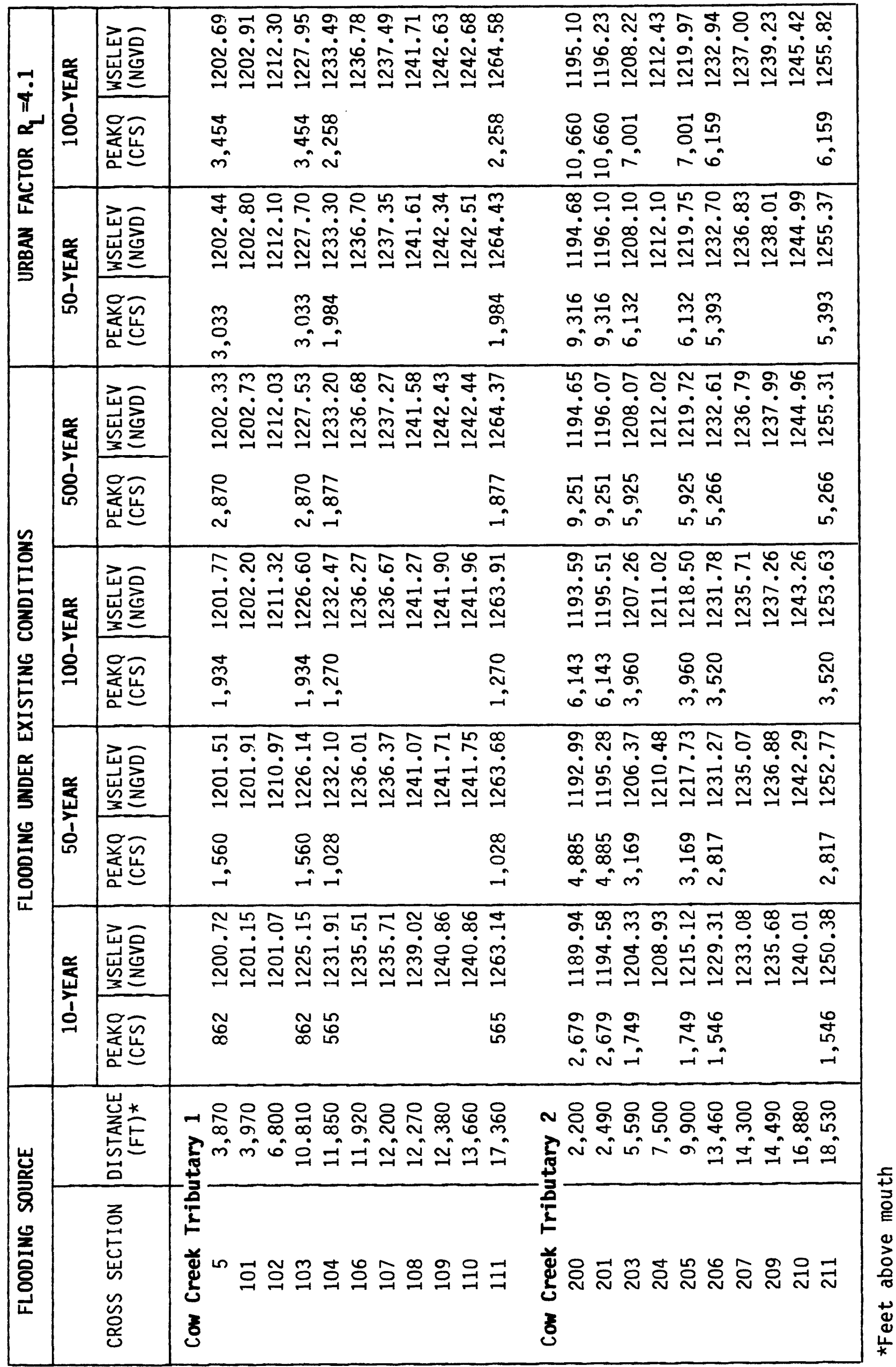




\begin{tabular}{|c|c|c|c|c|c|}
\hline \multirow{4}{*}{ 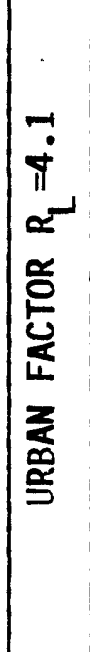 } & \multirow{2}{*}{ 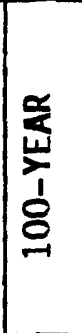 } & 岂司 & 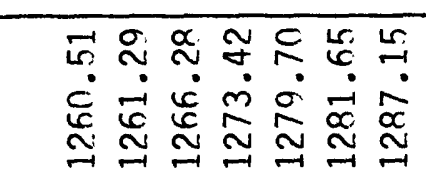 & 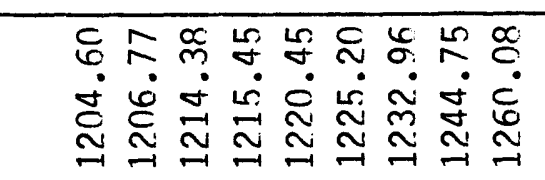 & 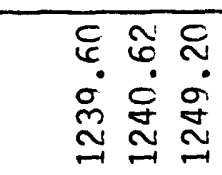 \\
\hline & & 总矛 & 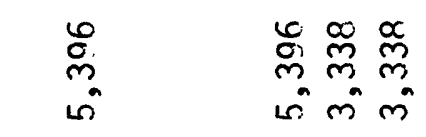 & $\begin{array}{lll}-1 & 0 & 0 \\
6 & 0 & 8 \\
6 & 0 \\
n & \sim & n\end{array}$ & $\begin{array}{ll}\stackrel{L}{\sim} & \stackrel{L}{\sim} \\
\stackrel{\infty}{\sim} & \sim\end{array}$ \\
\hline & 产 & 崖至 & 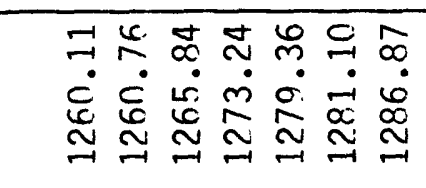 & 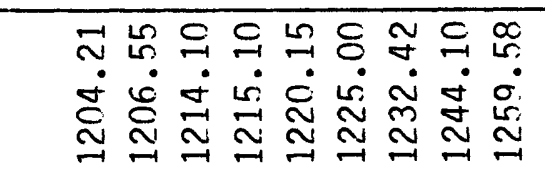 & 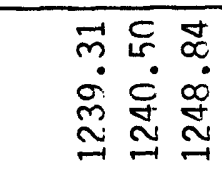 \\
\hline & 官 & 盖总 & 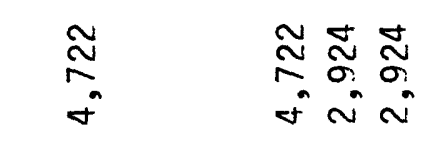 & 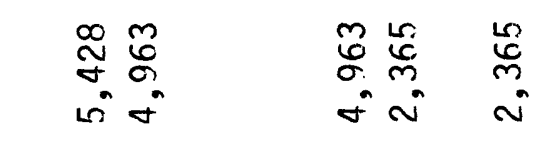 & \begin{tabular}{ll}
$\stackrel{\infty}{*}$ & $\infty$ \\
\multirow{f}{\sigma}{} & $\stackrel{\sim}{\sim}$ \\
$\sim$ & $\sim$
\end{tabular} \\
\hline & 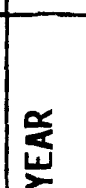 & 崖 & 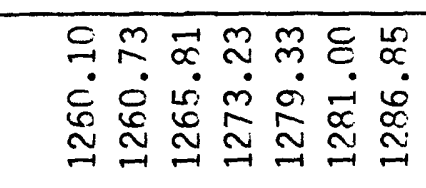 & 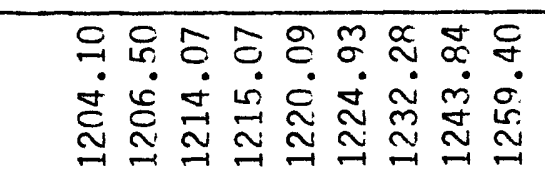 & 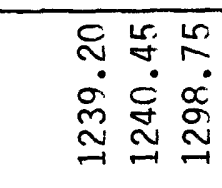 \\
\hline & ¿̊ & 善苍 & 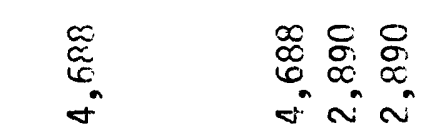 & 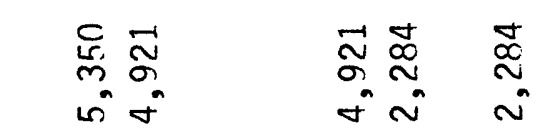 & 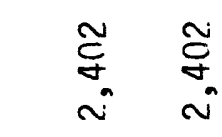 \\
\hline 它 & 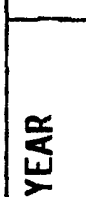 & 崫导 & 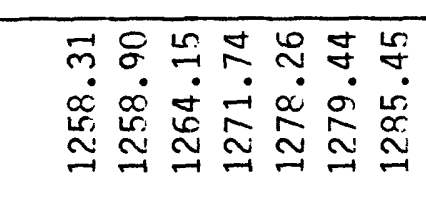 & 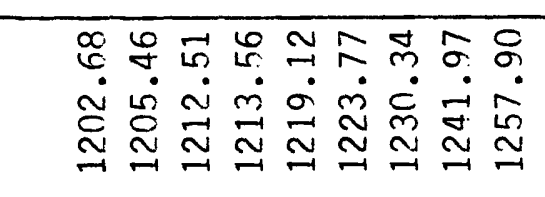 & 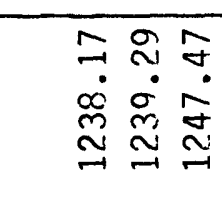 \\
\hline$\underset{\Xi}{E}$ & $\stackrel{1}{\varrho}$ & 席总 & $\begin{array}{ll}\vec{m} & \vec{m} \\
\vec{m} & \dot{\sigma} \sigma \\
\dot{m} & m-i\end{array}$ & 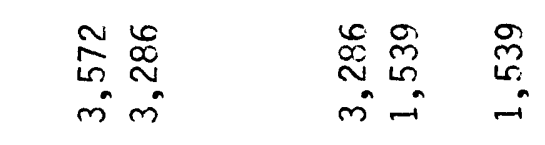 & $\begin{array}{ll}\underset{\infty}{c} & \stackrel{\infty}{b} \\
\stackrel{-}{-} & -\end{array}$ \\
\hline $\begin{array}{l}\text { 甾 } \\
\text { 芘 } \\
\frac{\partial}{5}\end{array}$ & 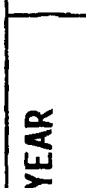 & 岂意 & 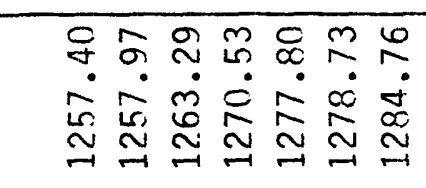 & 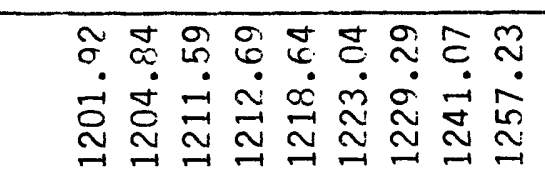 & 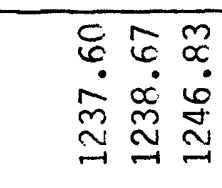 \\
\hline گ્ટ & 㐭 & 羞芯 & 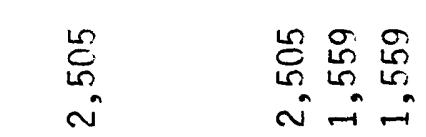 & 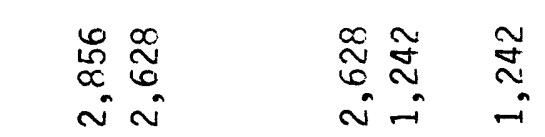 & 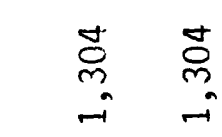 \\
\hline$\overline{4}$ & 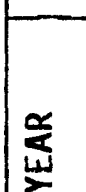 & 崖全 & 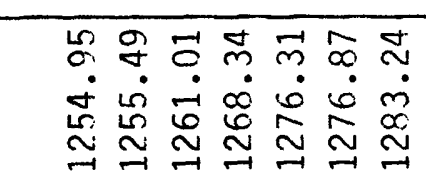 & 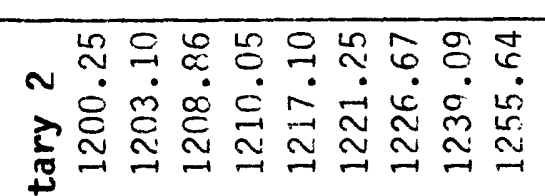 & 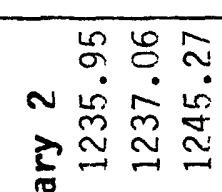 \\
\hline & $\stackrel{1}{\stackrel{1}{-1}}$ & 递苍 & 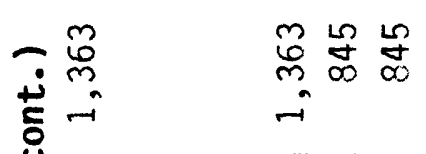 & 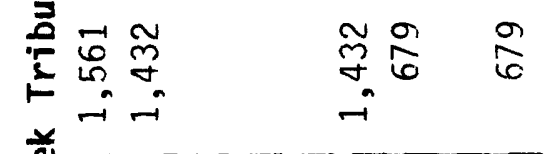 & 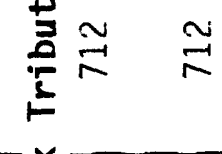 \\
\hline 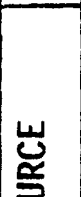 & & 岕岕 & 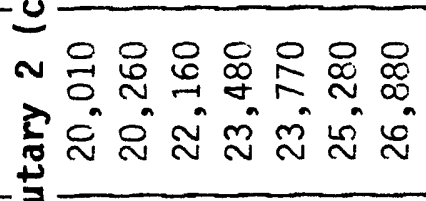 & 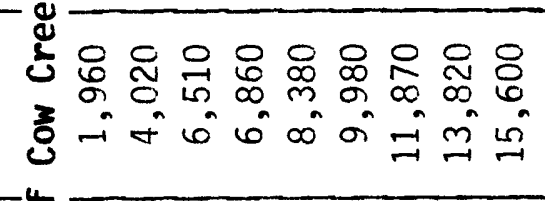 & 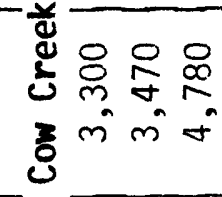 \\
\hline 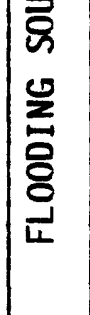 & & 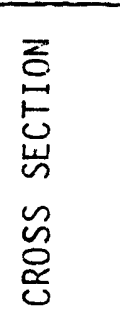 & 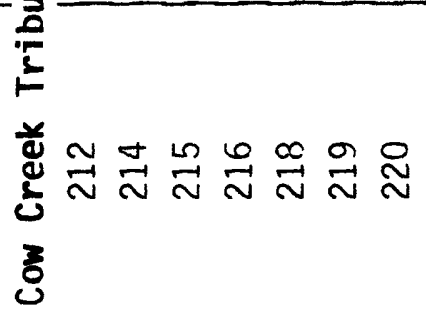 & 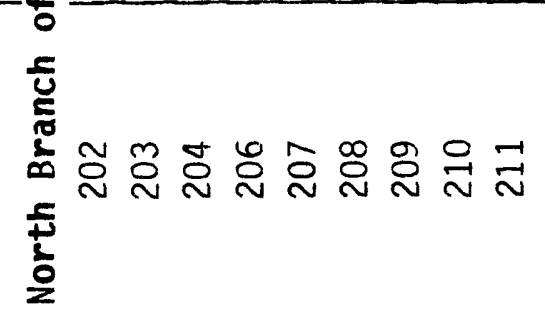 & 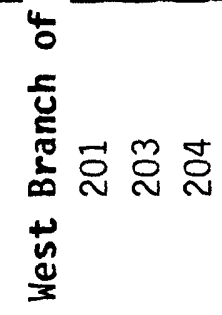 \\
\hline
\end{tabular}




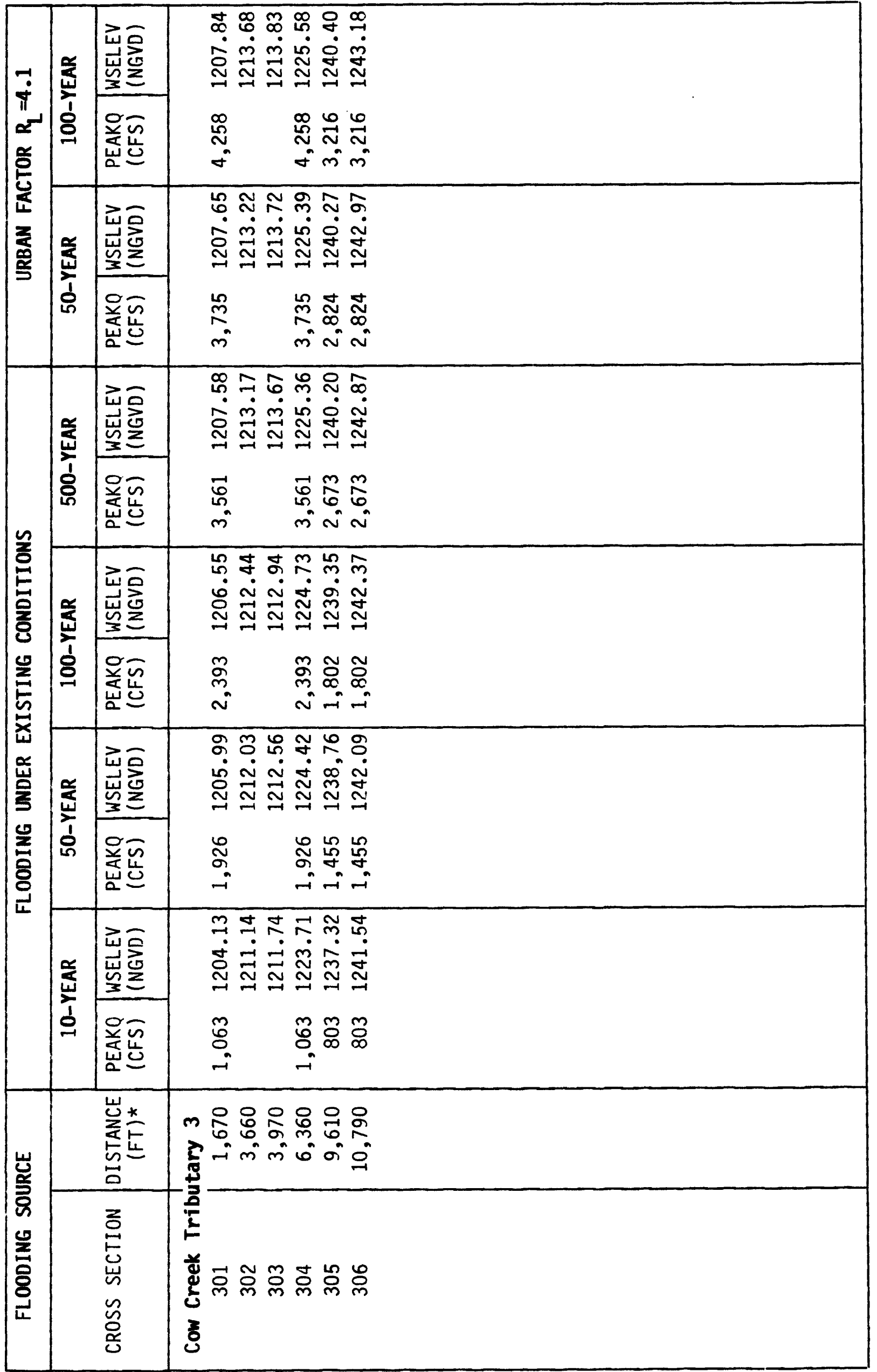




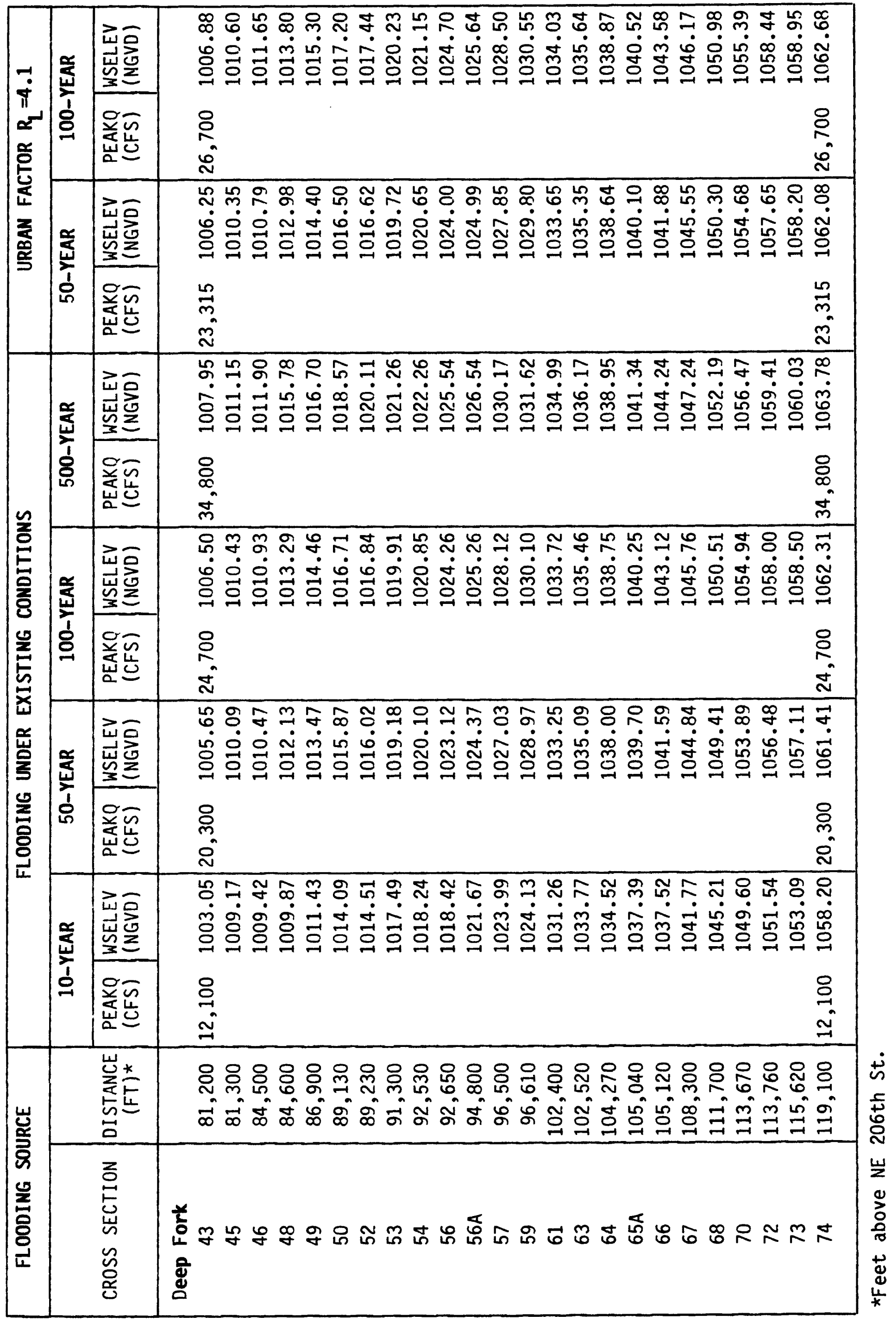




\begin{tabular}{|c|c|c|c|}
\hline \multirow{3}{*}{ 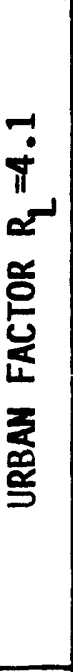 } & 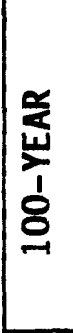 & 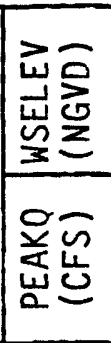 & 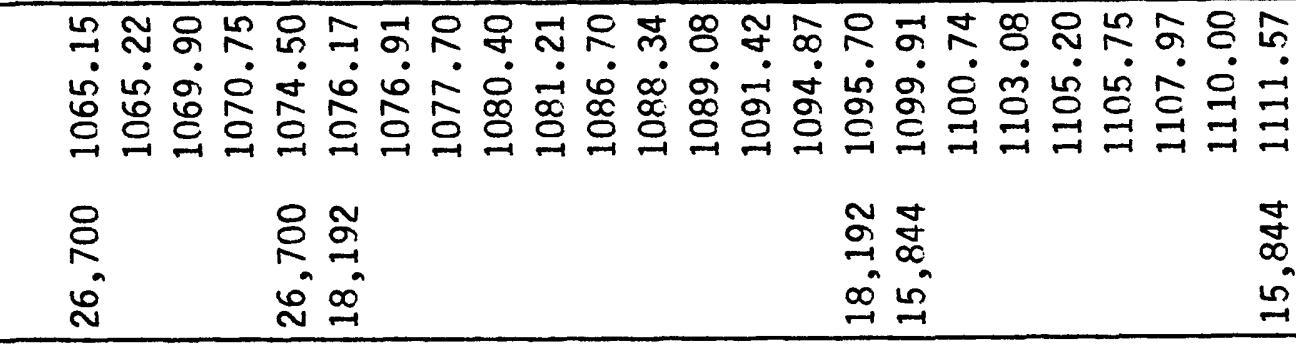 \\
\hline & \multirow{2}{*}{$\mid \begin{array}{l}\frac{\alpha}{5} \\
\frac{1}{1} \\
\frac{1}{L}\end{array}$} & 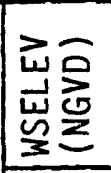 & 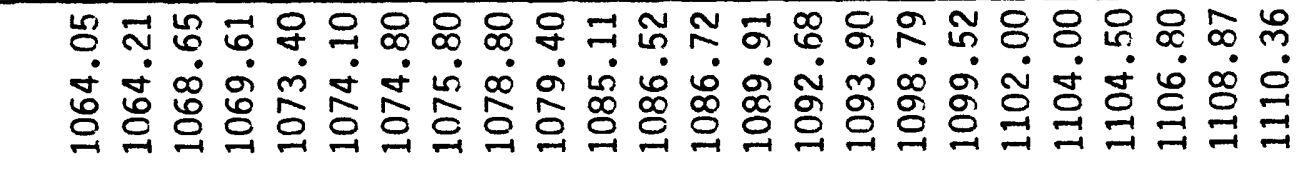 \\
\hline & & $\begin{array}{l}\text { 恙 } \\
\text { 岂至 }\end{array}$ & 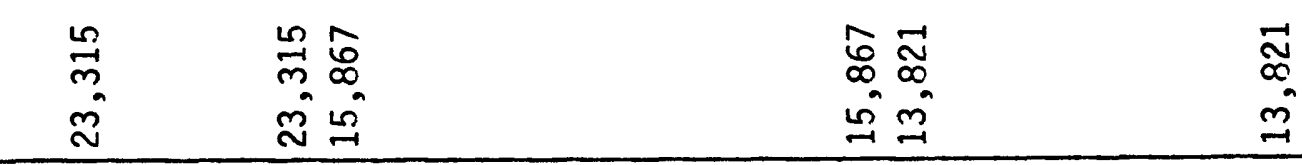 \\
\hline \multirow{8}{*}{ 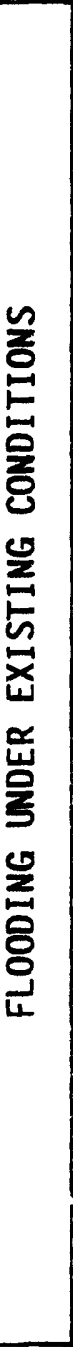 } & \multirow{2}{*}{$\begin{array}{l}\frac{x}{4} \\
\frac{1}{1} \\
\frac{1}{0}\end{array}$} & 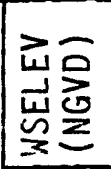 & 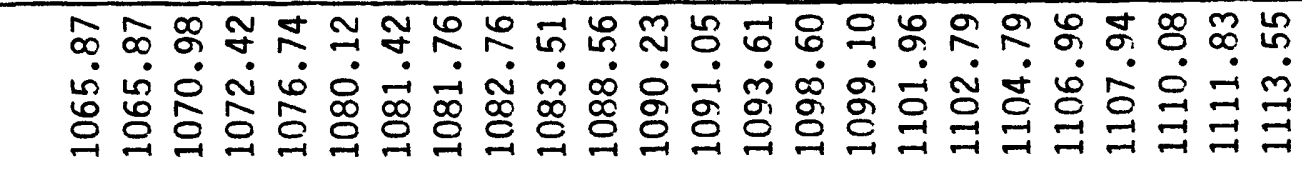 \\
\hline & & 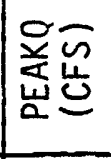 & 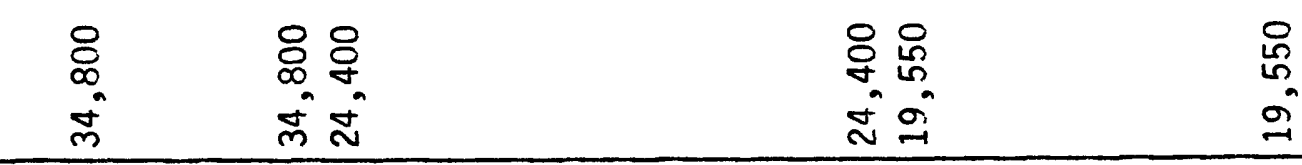 \\
\hline & \multirow{2}{*}{ 产 } & 崖邑 & 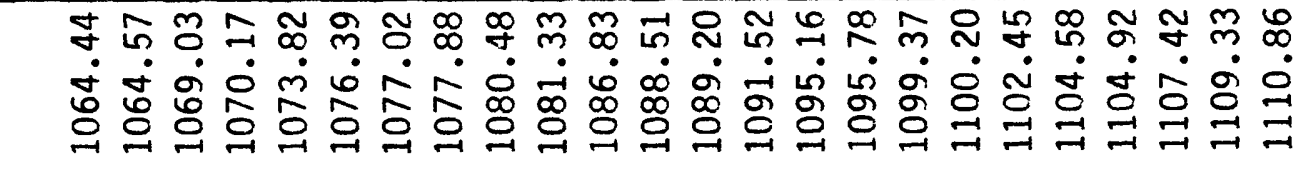 \\
\hline & & 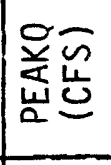 & 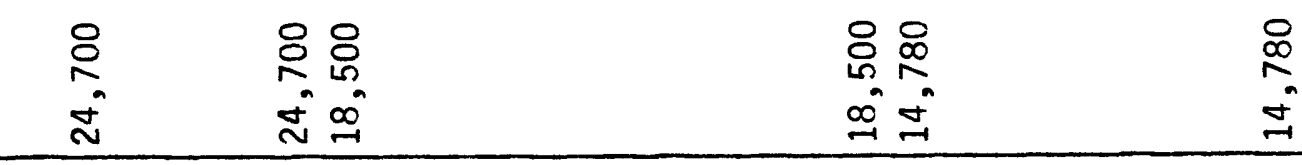 \\
\hline & \multirow{2}{*}{$\frac{\infty}{\frac{1}{1}}$} & 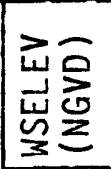 & 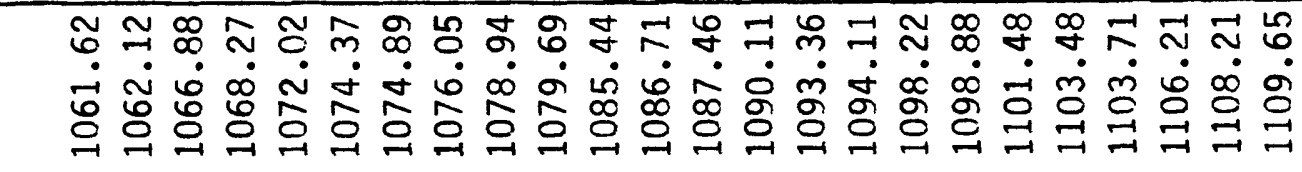 \\
\hline & & $\begin{array}{l}\text { 을 } \\
\text { 岂㫐 }\end{array}$ & 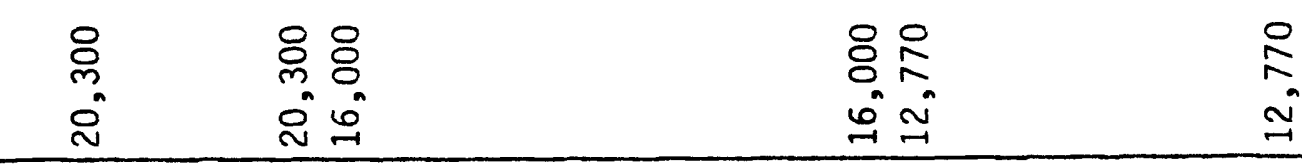 \\
\hline & \multirow{2}{*}{ 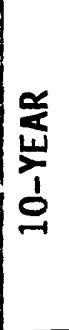 } & 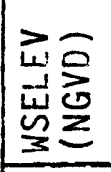 & 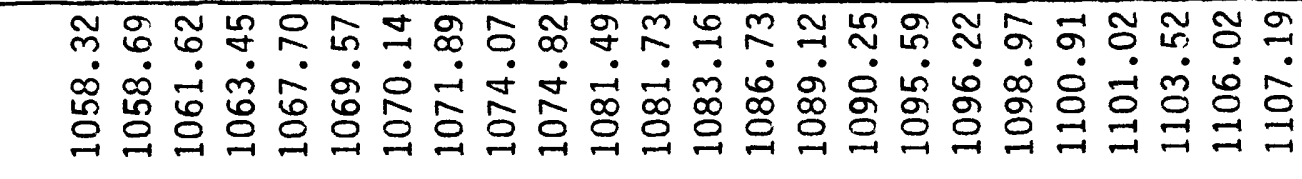 \\
\hline & & 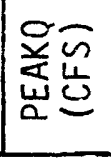 & 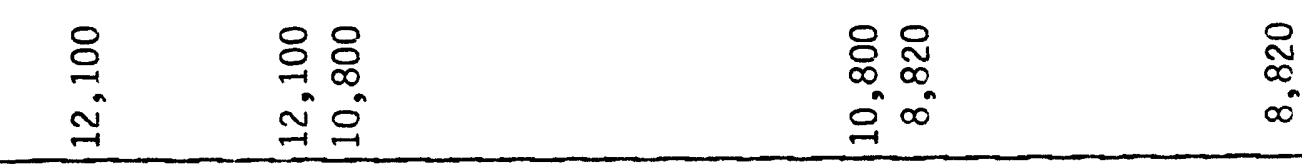 \\
\hline \multirow{2}{*}{ 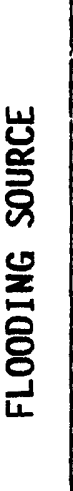 } & & 岕岕 & 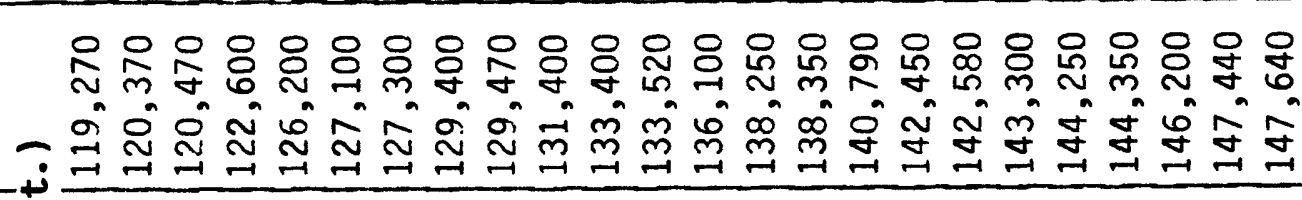 \\
\hline & & 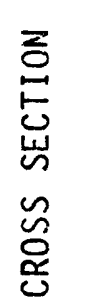 & 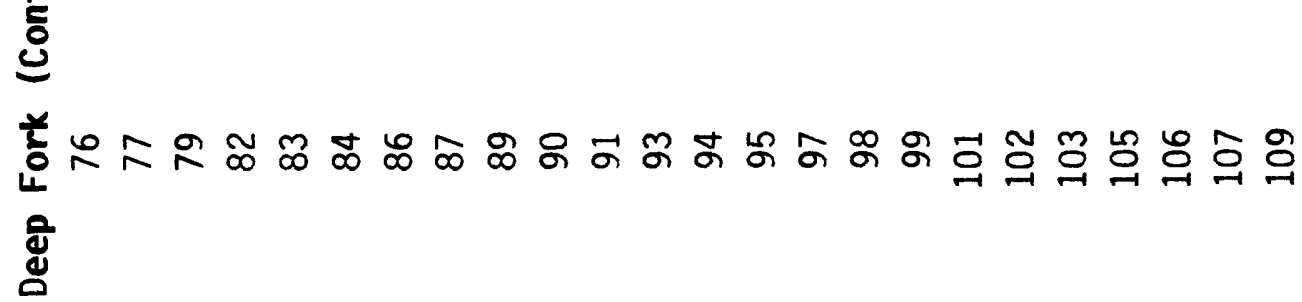 \\
\hline
\end{tabular}




\begin{tabular}{|c|c|c|c|}
\hline \multirow{3}{*}{ 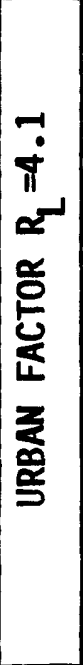 } & 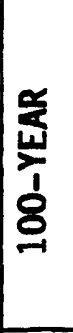 & 崖导 & 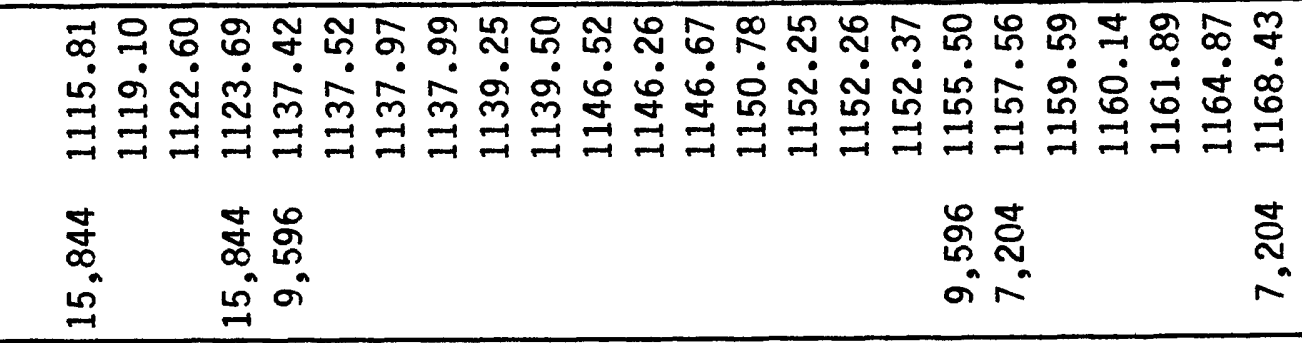 \\
\hline & \multirow{2}{*}{$\frac{⿱}{\mathbf{c}}$} & 崖 & 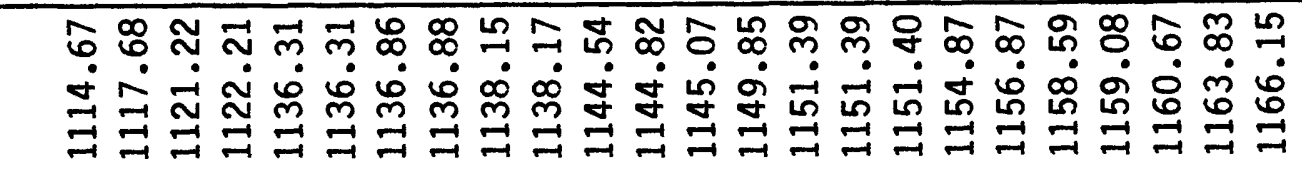 \\
\hline & & 并出 & 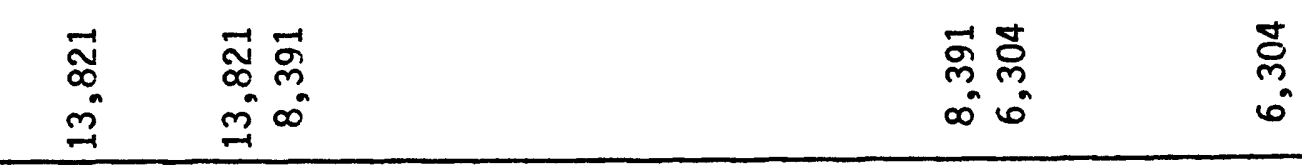 \\
\hline \multirow{8}{*}{ 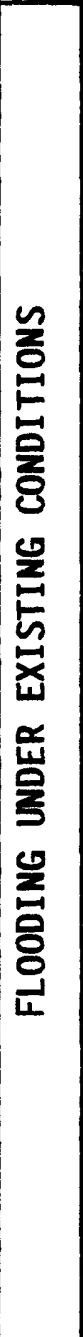 } & \multirow{2}{*}{$\frac{\infty}{\grave{c}}$} & 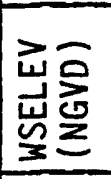 & 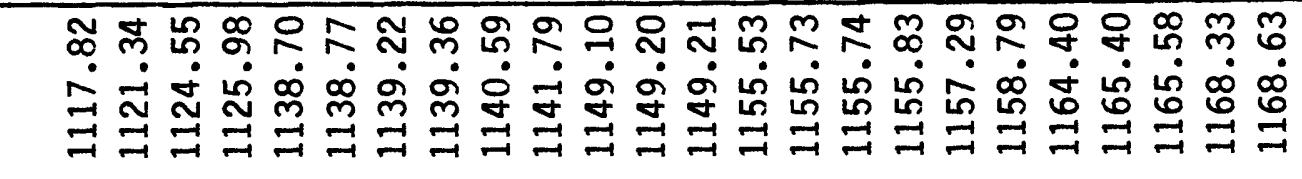 \\
\hline & & 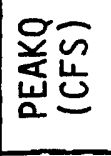 & 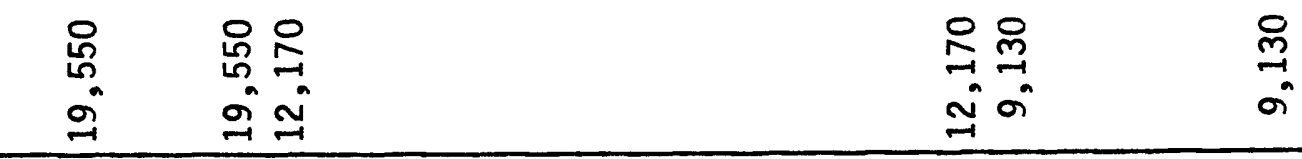 \\
\hline & \multirow{2}{*}{ 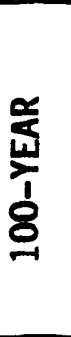 } & 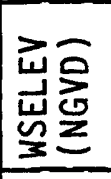 & 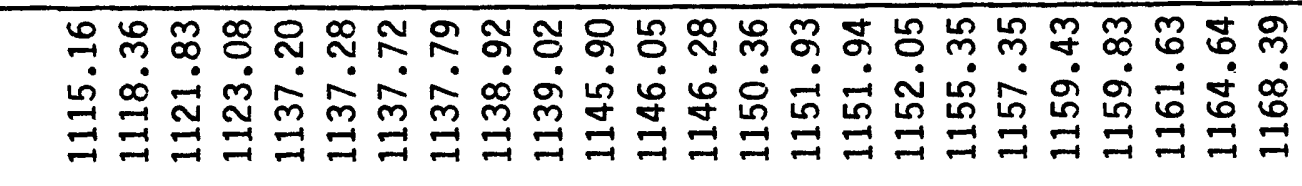 \\
\hline & & 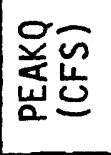 & 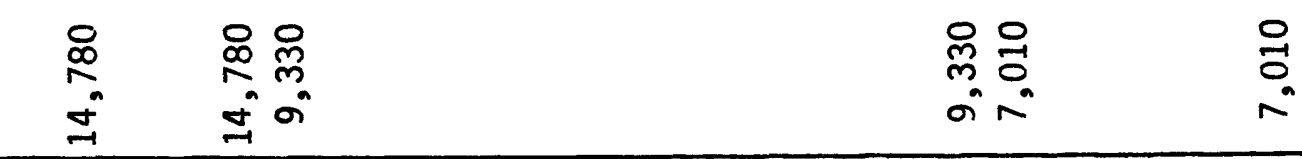 \\
\hline & \multirow{2}{*}{$\frac{x}{\frac{1}{1}}$} & 崖岕 & 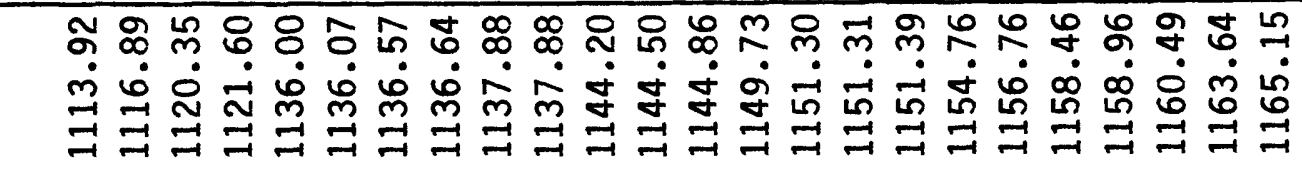 \\
\hline & & 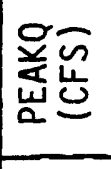 & 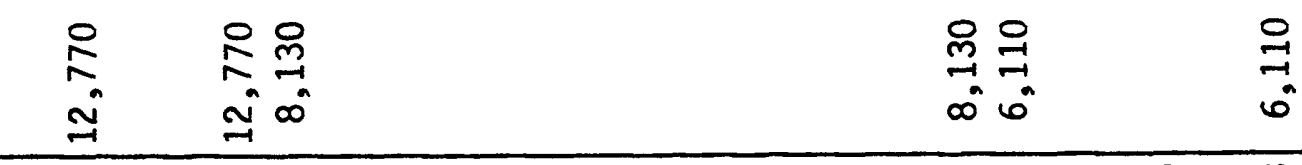 \\
\hline & \multirow{2}{*}{$\frac{0}{\frac{0}{5}}$} & 崖岕 & 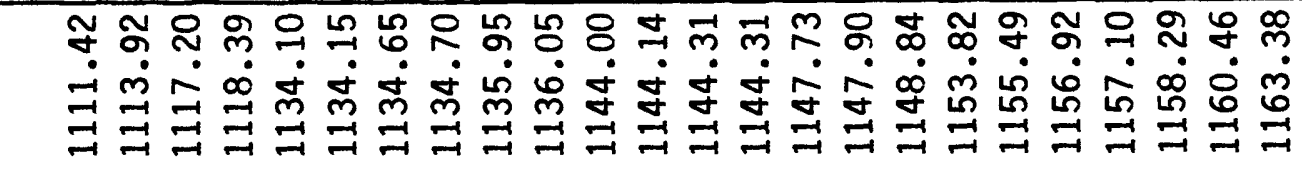 \\
\hline & & $\begin{array}{l}\text { 怘出 } \\
\text { 岂出 }\end{array}$ & 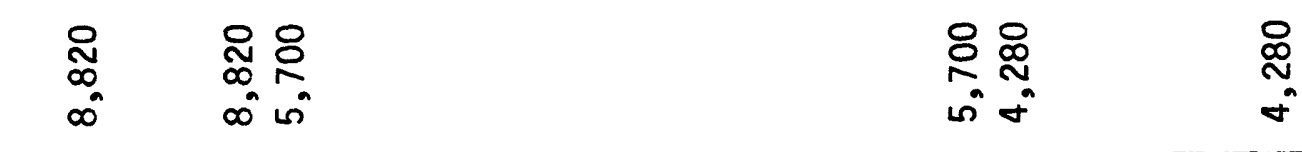 \\
\hline \multirow{2}{*}{ 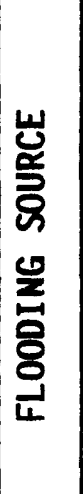 } & & 岕岕 & 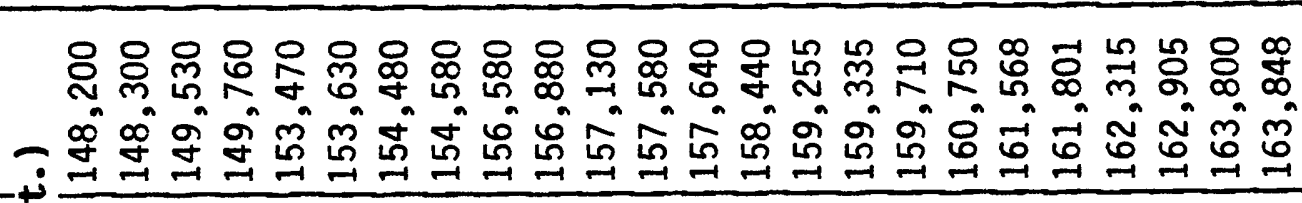 \\
\hline & & 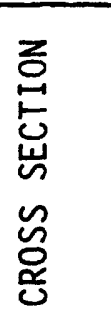 & 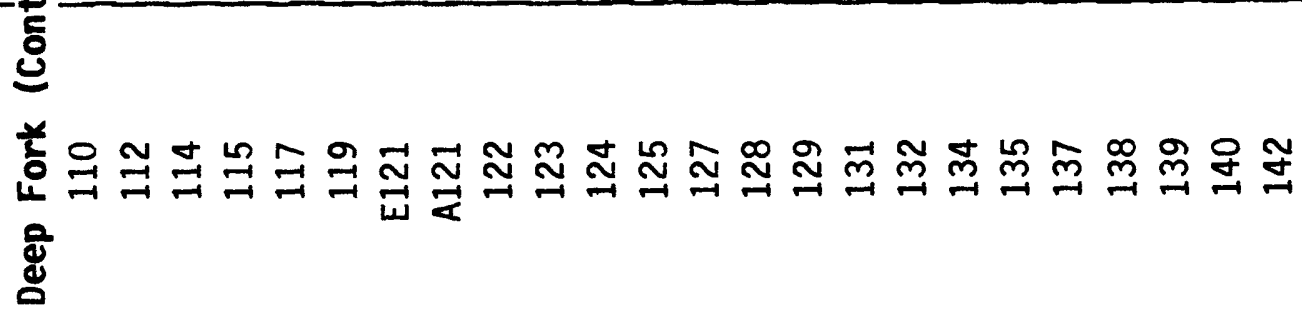 \\
\hline
\end{tabular}




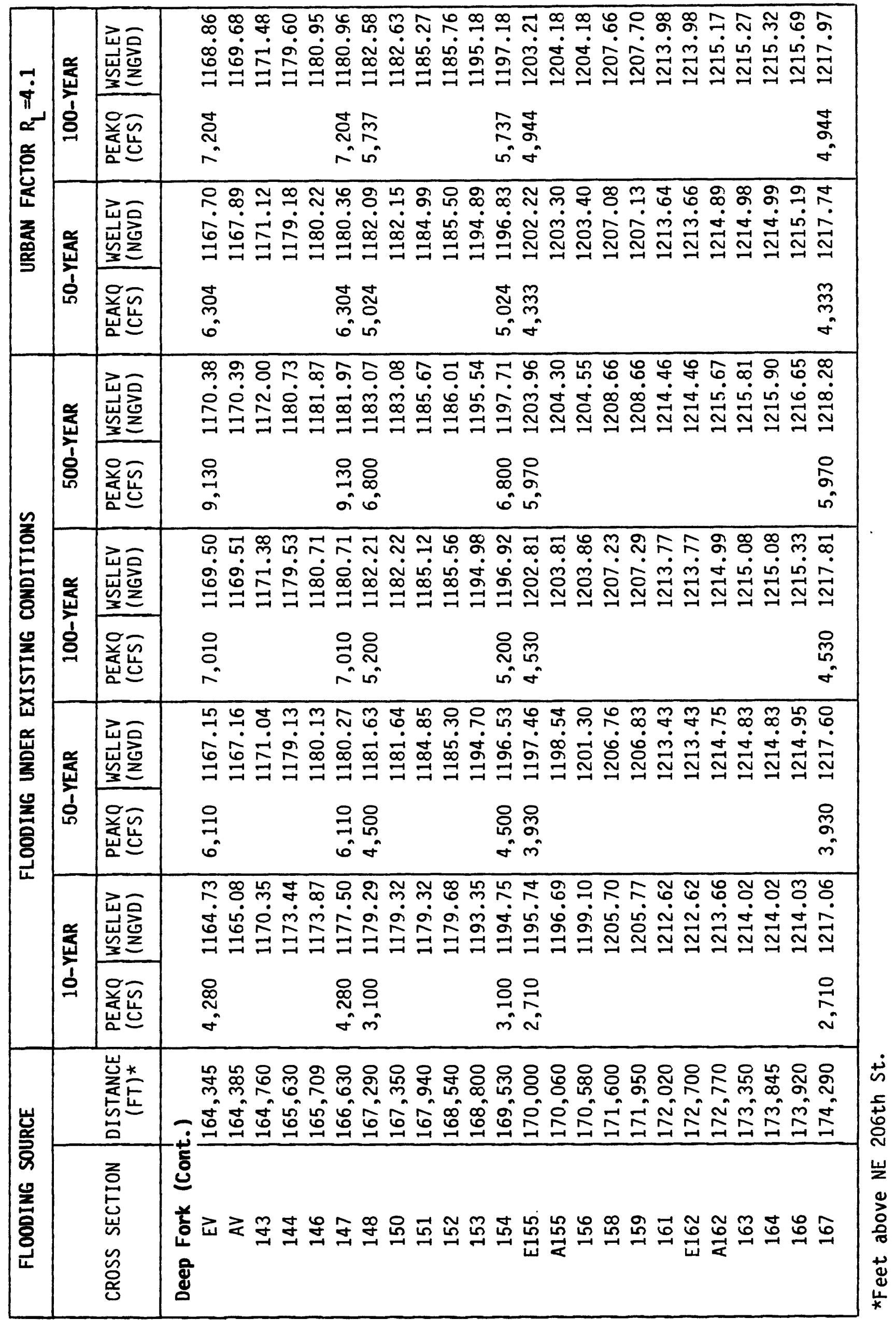




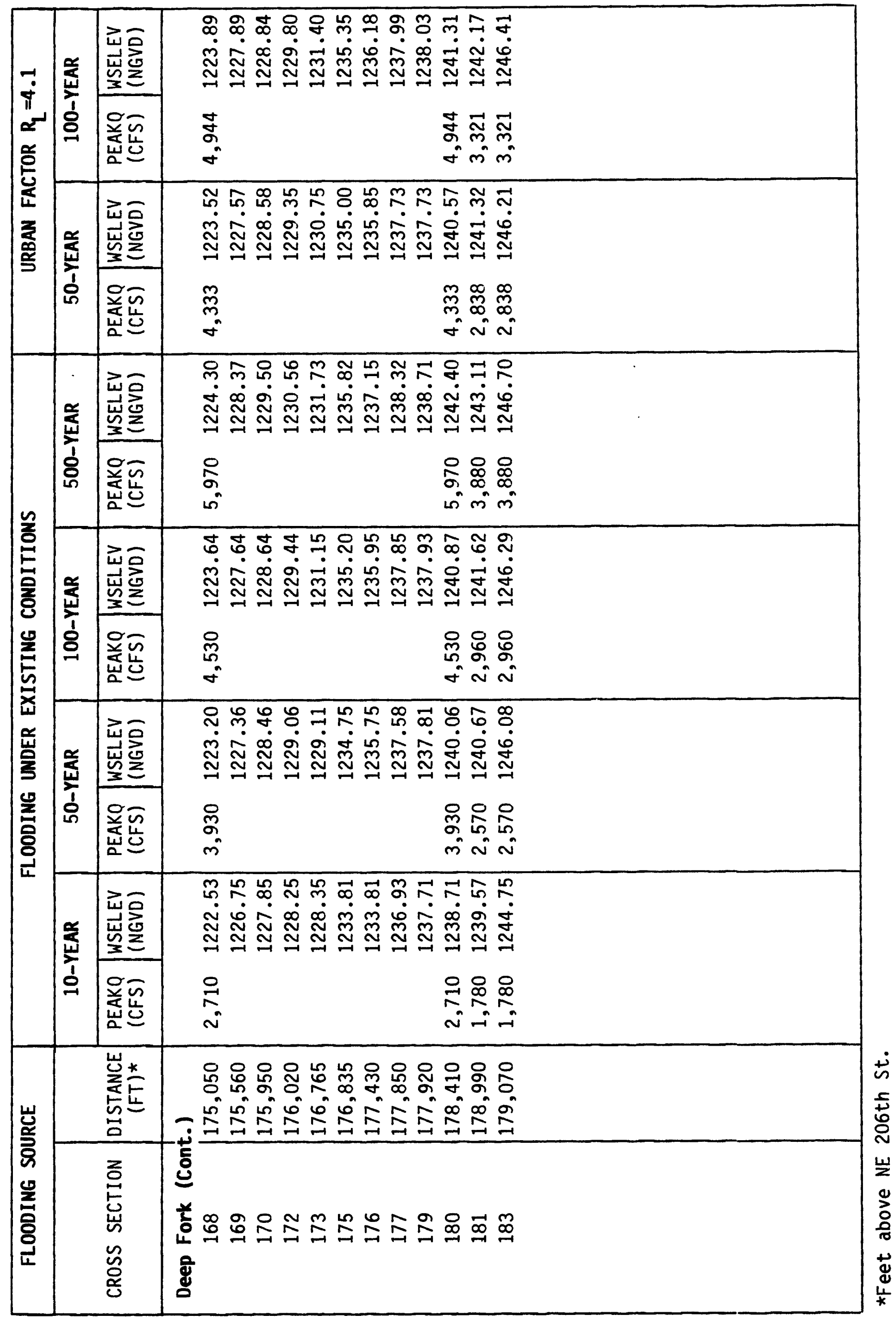




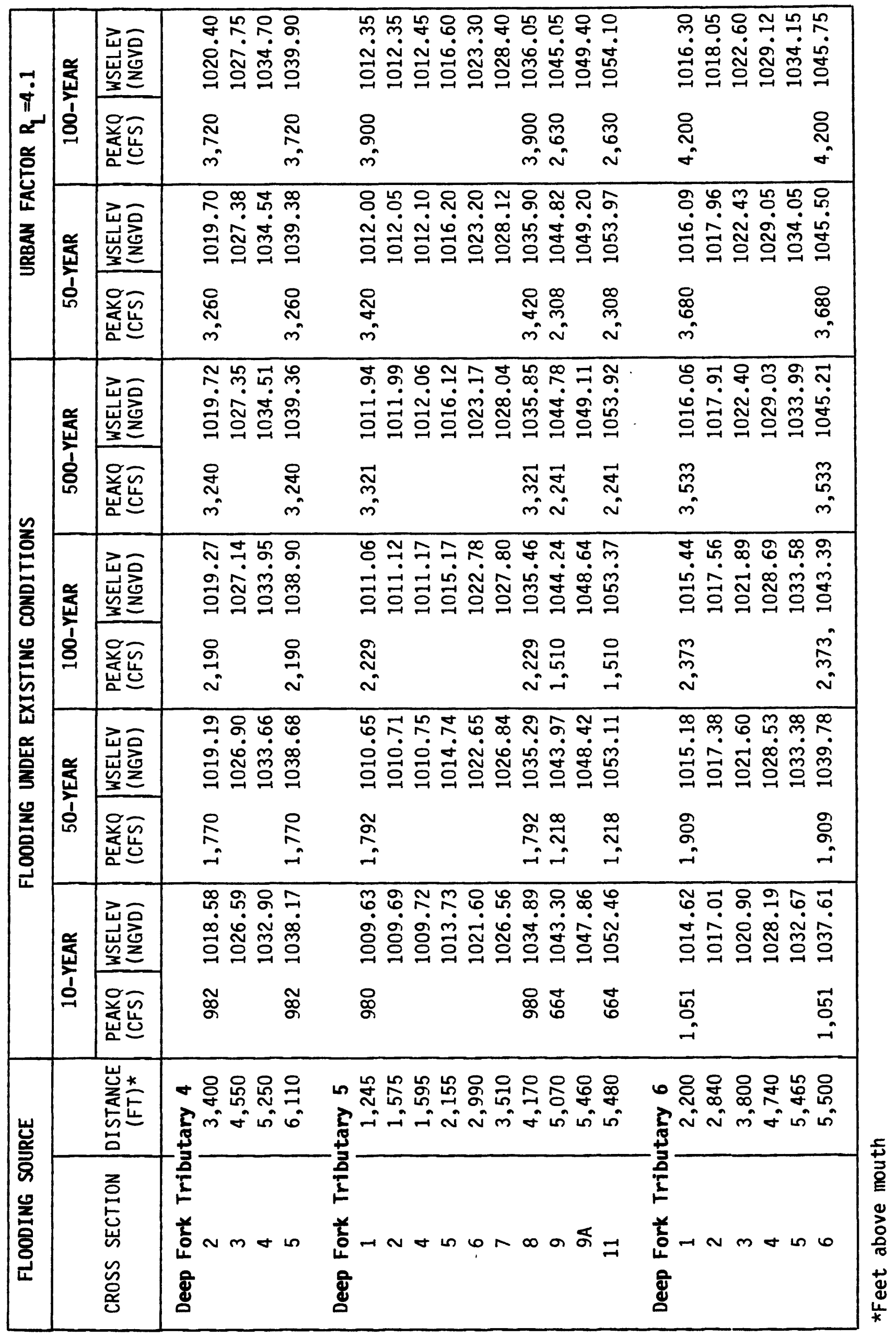




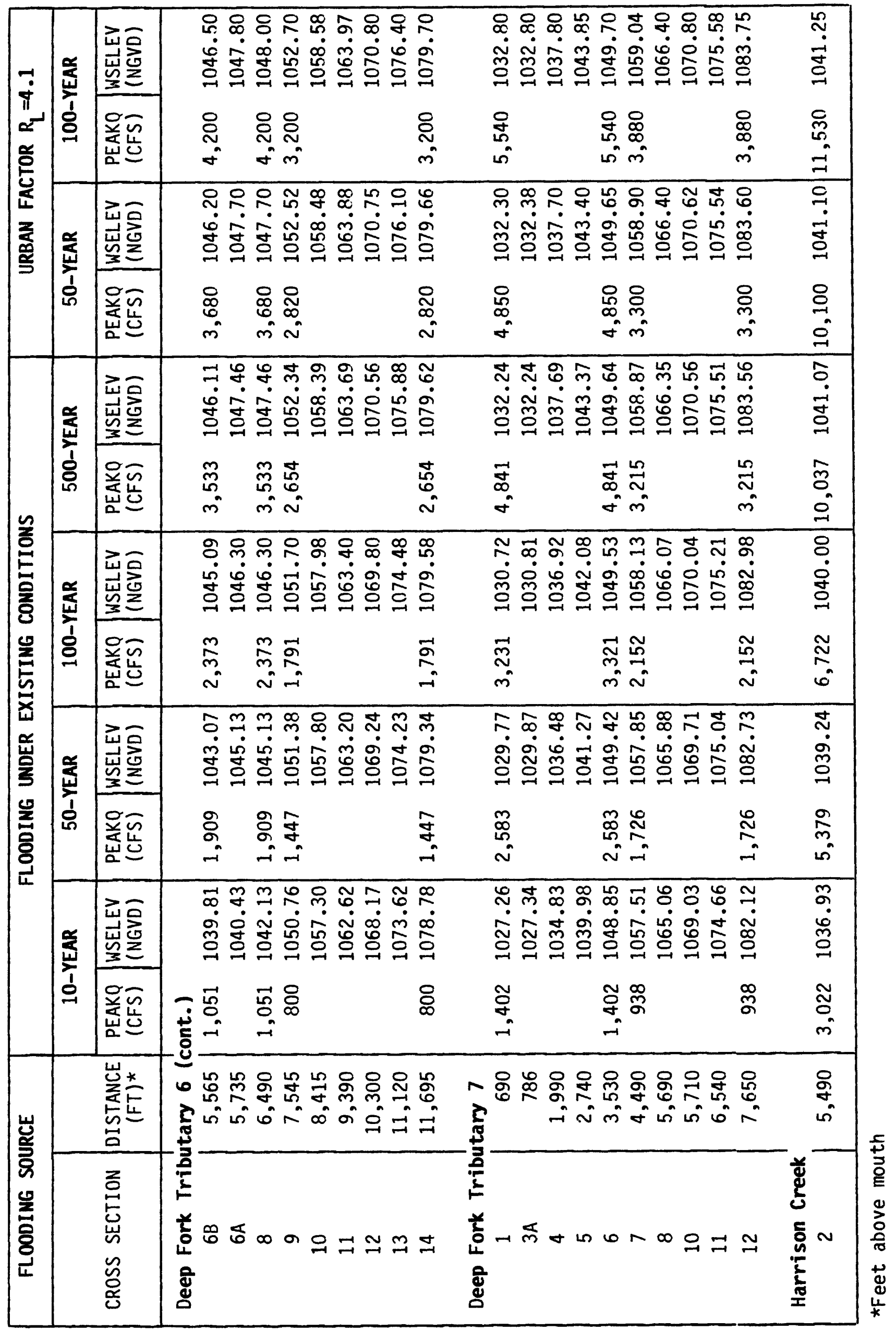




\begin{tabular}{|c|c|c|c|}
\hline \multirow{3}{*}{ 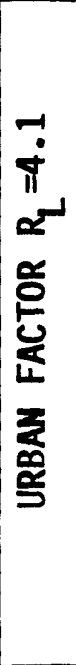 } & 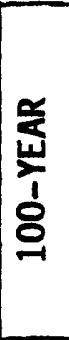 & 岂量 & 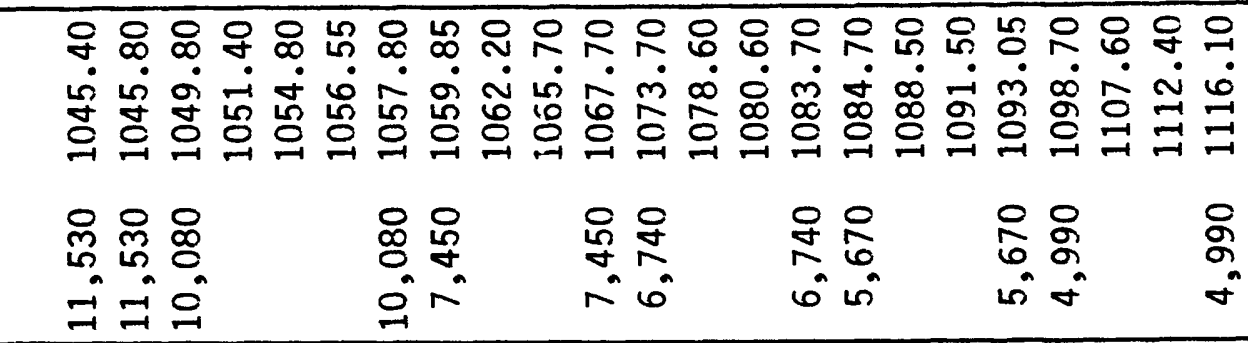 \\
\hline & \multirow{2}{*}{ 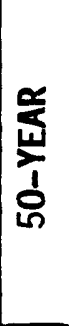 } & 崖 & 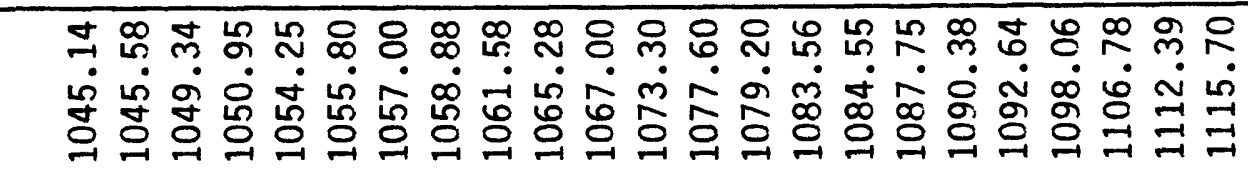 \\
\hline & & 递 & 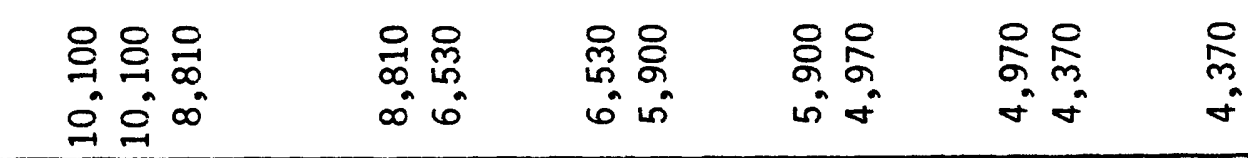 \\
\hline \multirow{8}{*}{ 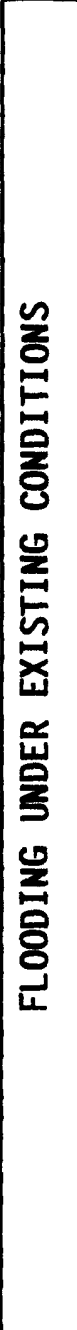 } & \multirow{2}{*}{ 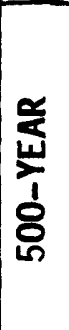 } & 崖 & 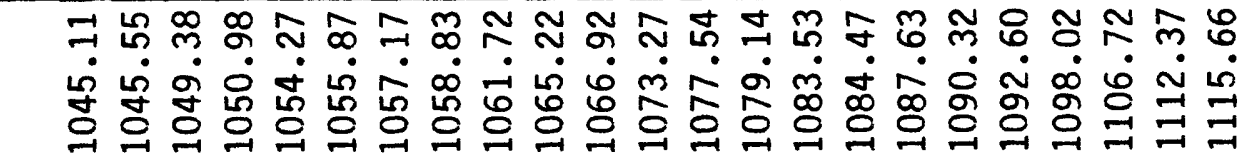 \\
\hline & & 盖苍 & 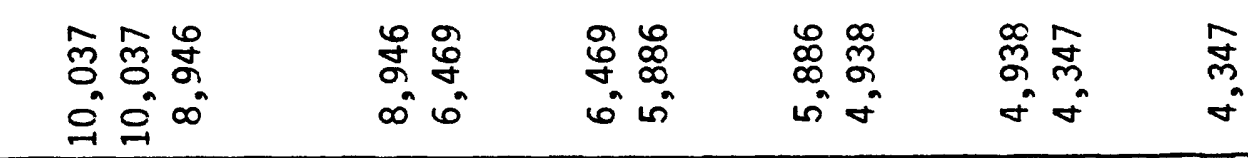 \\
\hline & \multirow{2}{*}{ 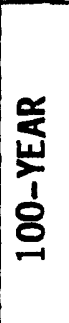 } & 崖 & 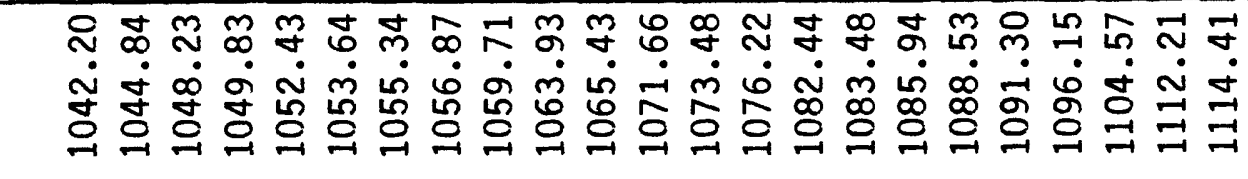 \\
\hline & & 递 & 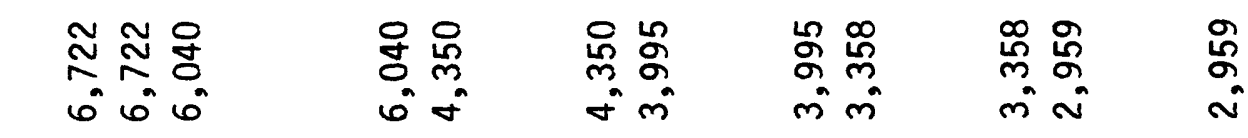 \\
\hline & \multirow{2}{*}{ 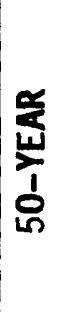 } & 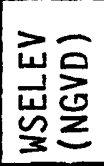 & 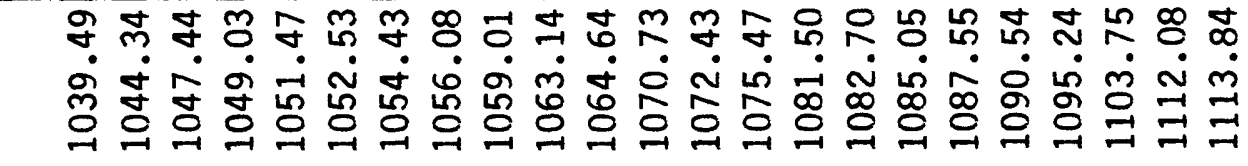 \\
\hline & & 递芯 & 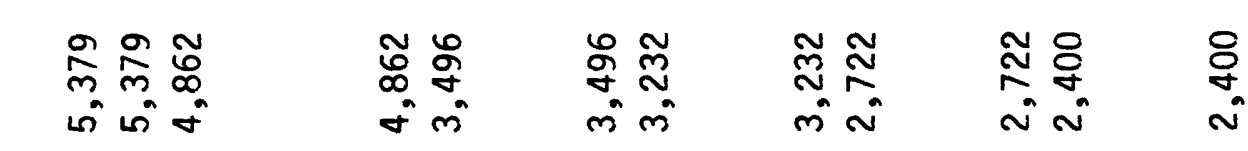 \\
\hline & \multirow{2}{*}{ 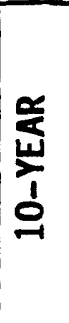 } & 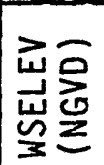 & 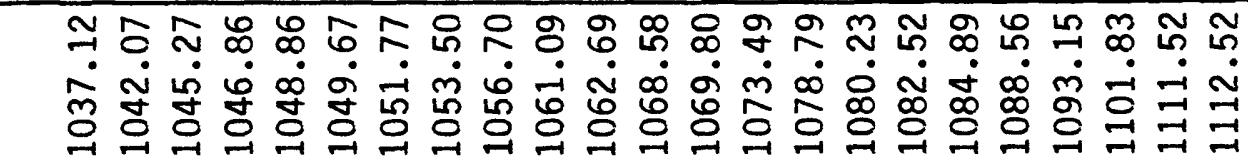 \\
\hline & & 羙苍 & 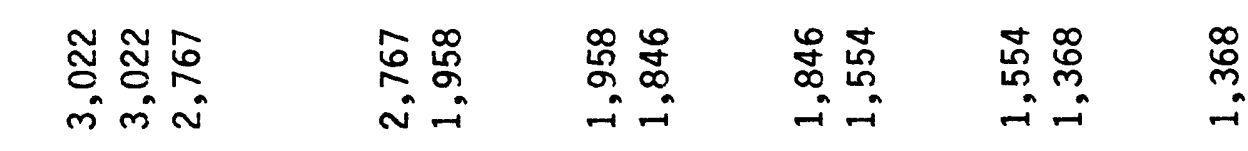 \\
\hline \multirow{2}{*}{ 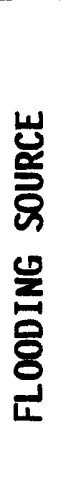 } & & 岂岕 & 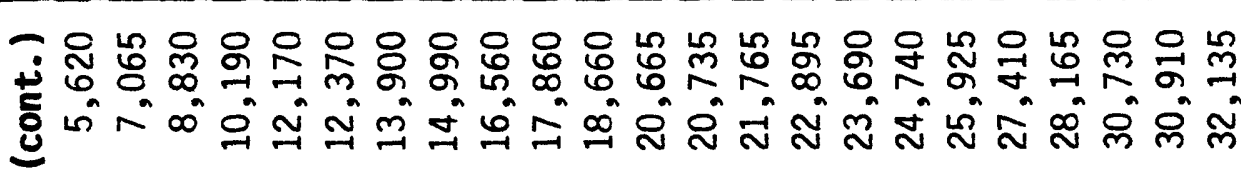 \\
\hline & & 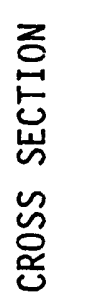 & 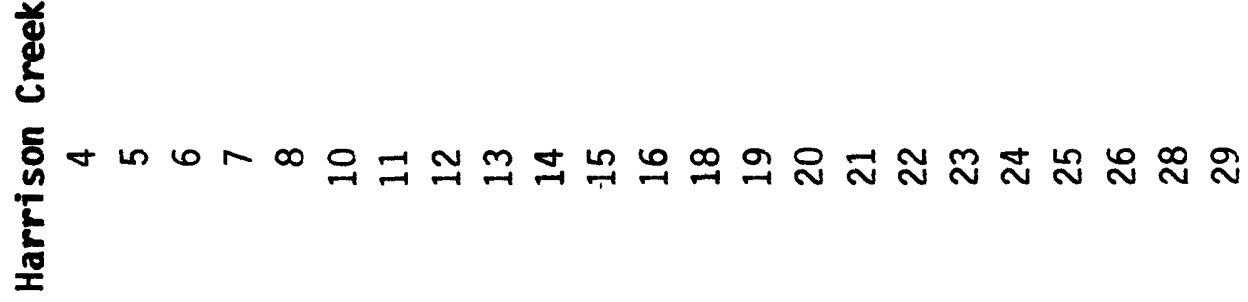 \\
\hline
\end{tabular}




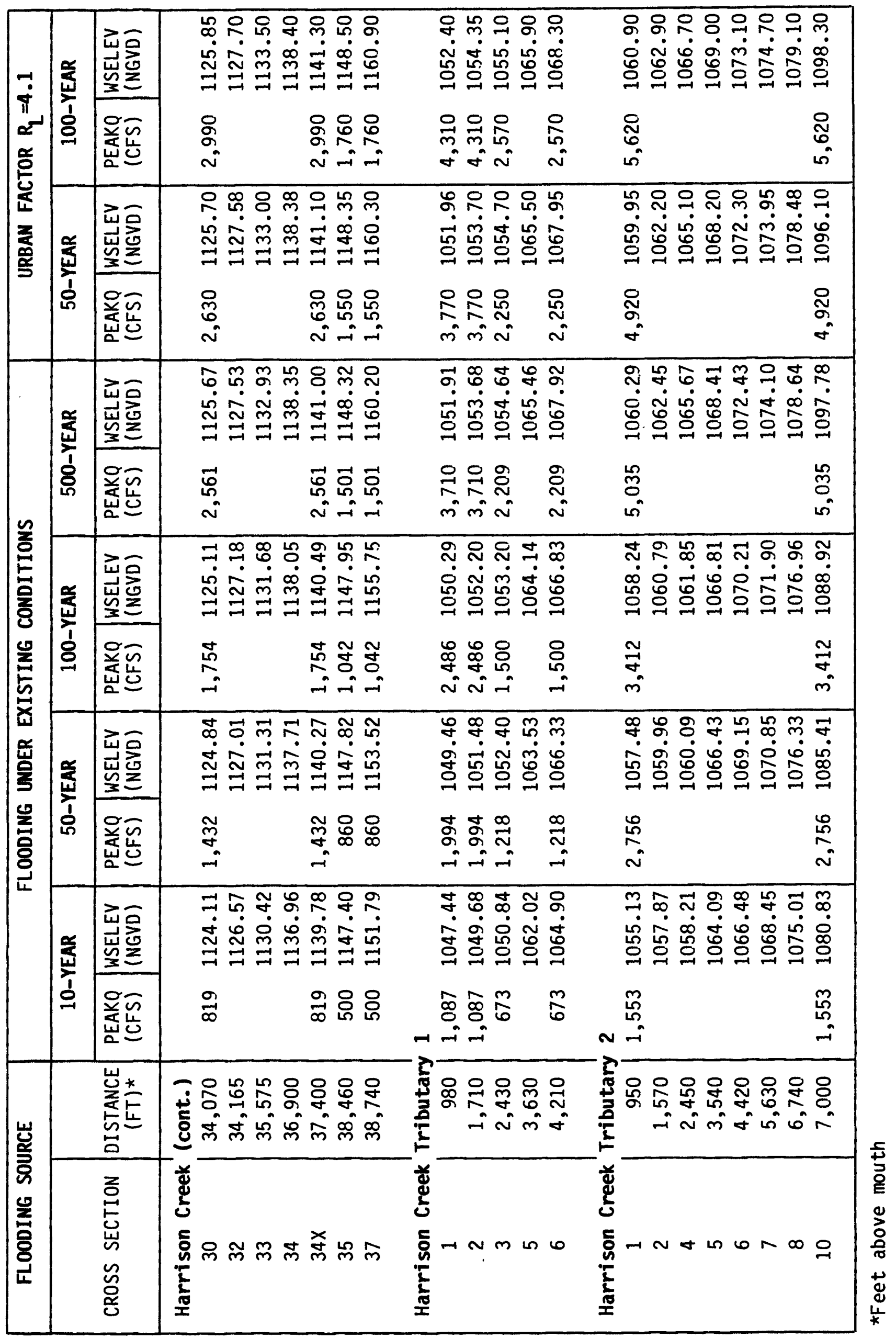




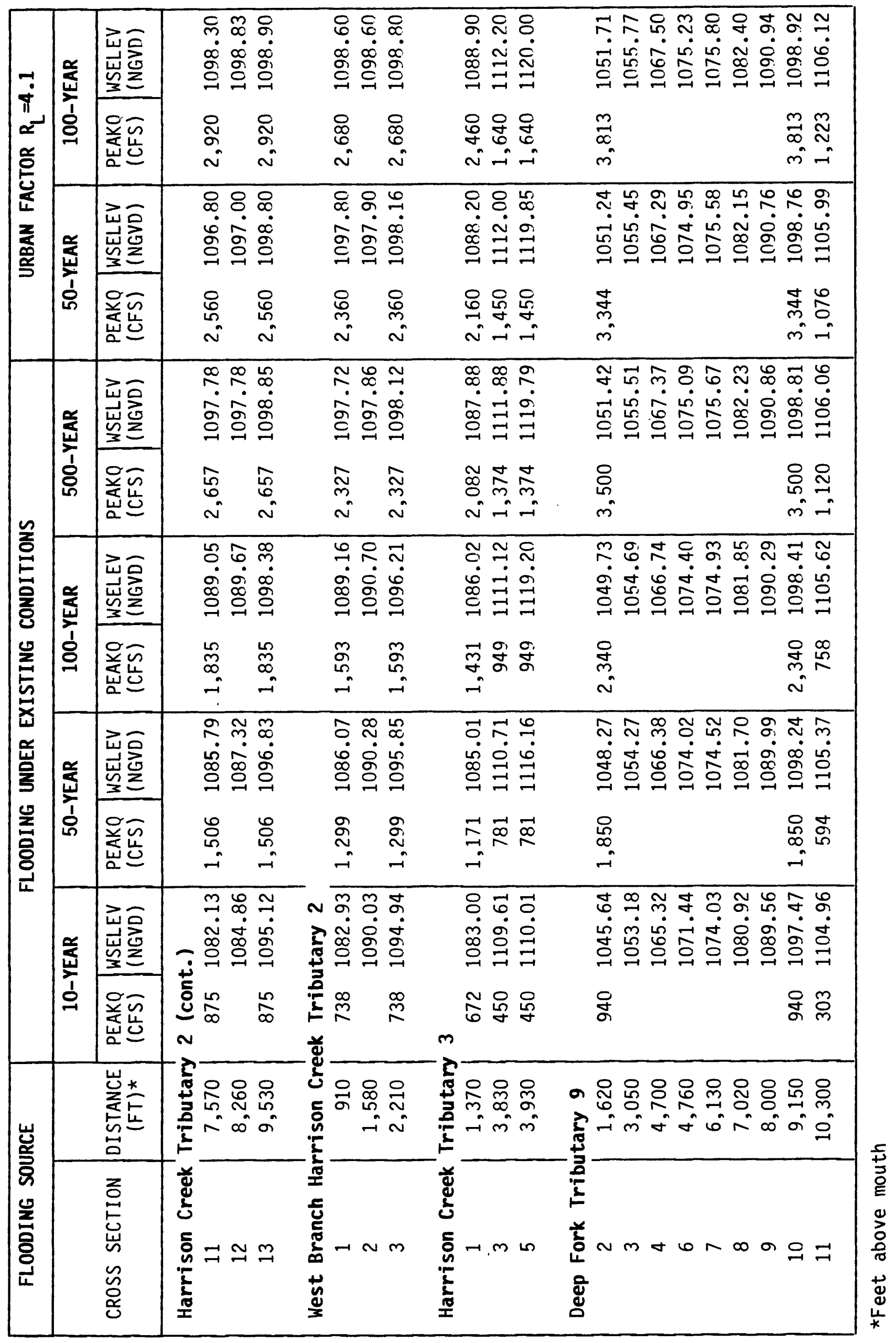




\begin{tabular}{|c|c|c|c|c|}
\hline \multirow{3}{*}{ 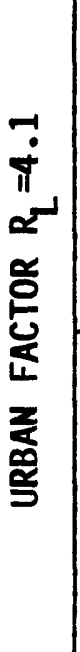 } & $\frac{0}{\frac{0}{5}}$ & 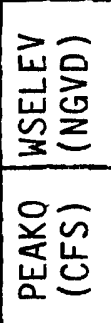 & 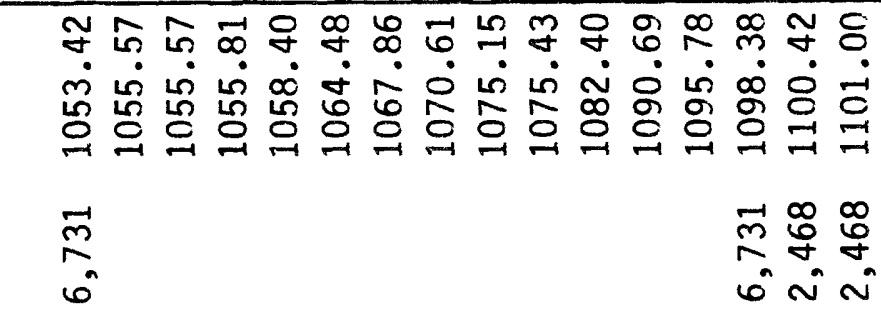 & 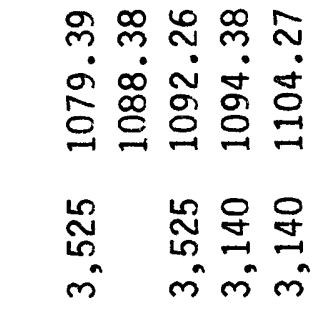 \\
\hline & \multirow{2}{*}{$\begin{array}{l}\frac{\alpha}{5} \\
\frac{1}{1} \\
0 \\
\end{array}$} & 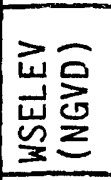 & \multirow{2}{*}{ 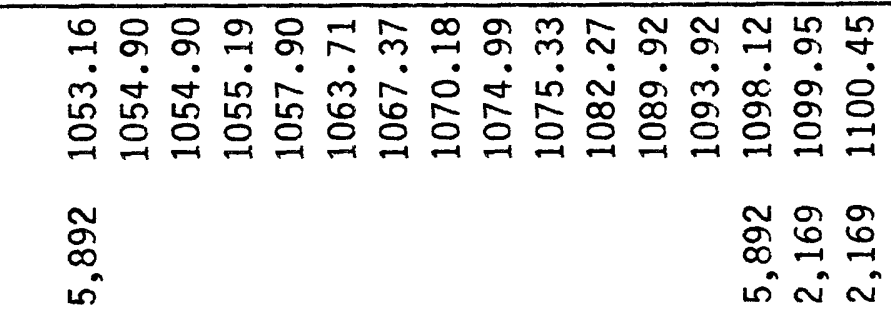 } & \multirow{2}{*}{ 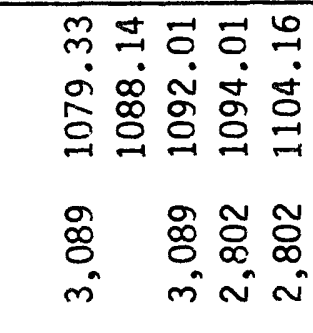 } \\
\hline & & 恙出 & & \\
\hline \multirow{8}{*}{ 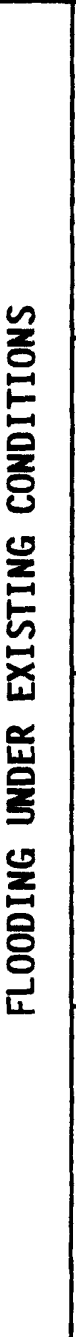 } & \multirow{2}{*}{$\begin{array}{l}\frac{1}{5} \\
\frac{1}{1} \\
\frac{1}{8} \\
\end{array}$} & 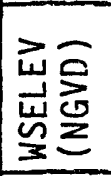 & \multirow{2}{*}{ 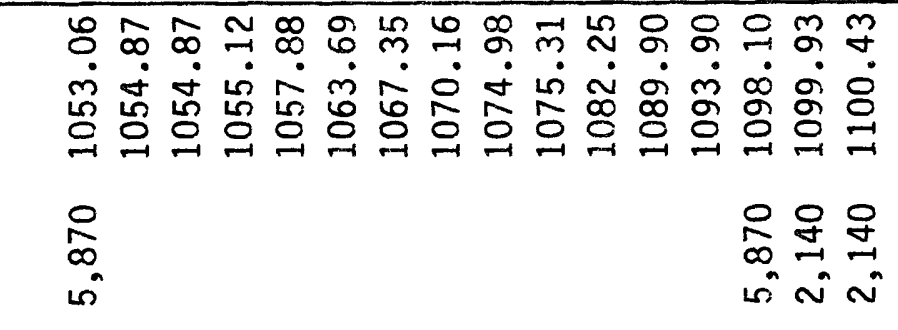 } & \multirow{2}{*}{ 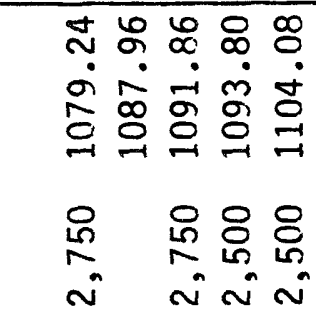 } \\
\hline & & $\begin{array}{l}\text { 弟 } \\
\text { 岂先 }\end{array}$ & & \\
\hline & \multirow{2}{*}{ 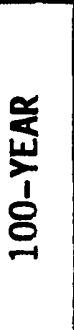 } & 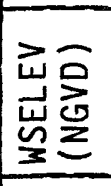 & \multirow{2}{*}{ 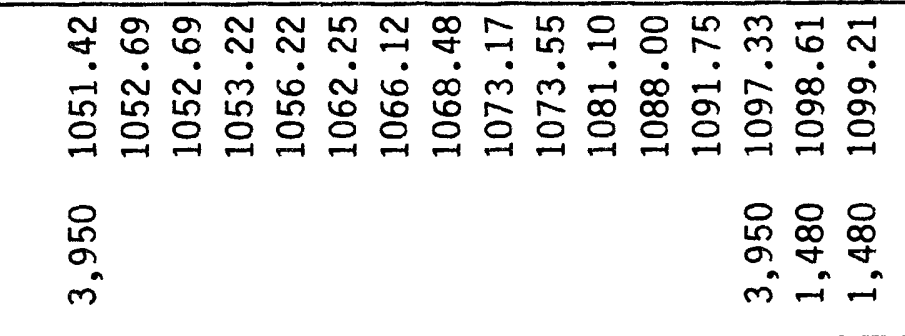 } & \multirow{2}{*}{ 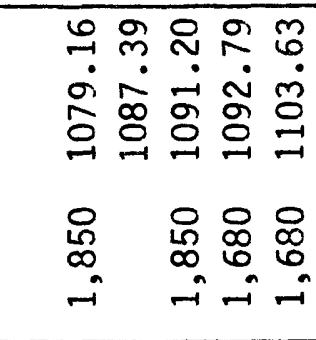 } \\
\hline & & 总苍 & & \\
\hline & \multirow{2}{*}{ 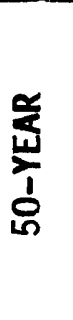 } & 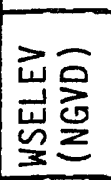 & \multirow{2}{*}{ 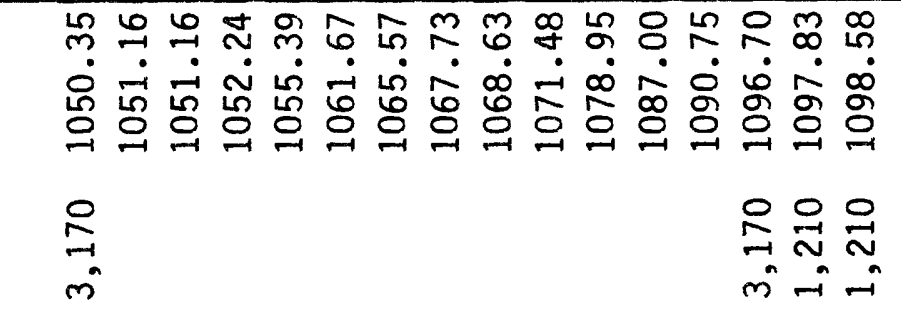 } & \multirow{2}{*}{ 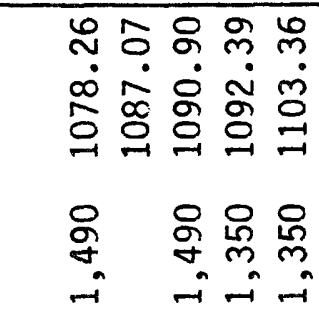 } \\
\hline & & 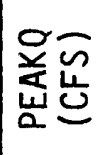 & & \\
\hline & \multirow{2}{*}{$\underset{\frac{\alpha}{5}}{\frac{\alpha}{b}}$} & 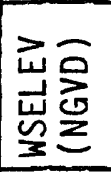 & \multirow{2}{*}{ 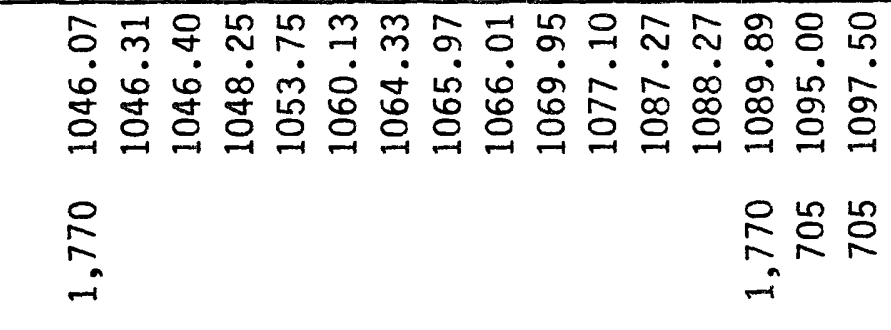 } & \multirow{2}{*}{ 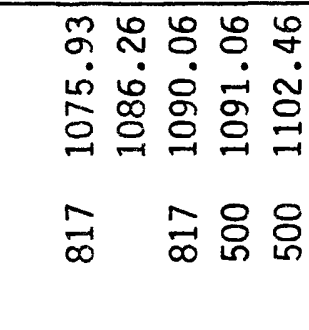 } \\
\hline & & 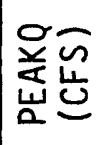 & & \\
\hline \multirow{2}{*}{ 岕 } & & 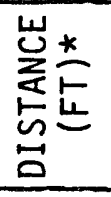 & \multirow[t]{2}{*}{ 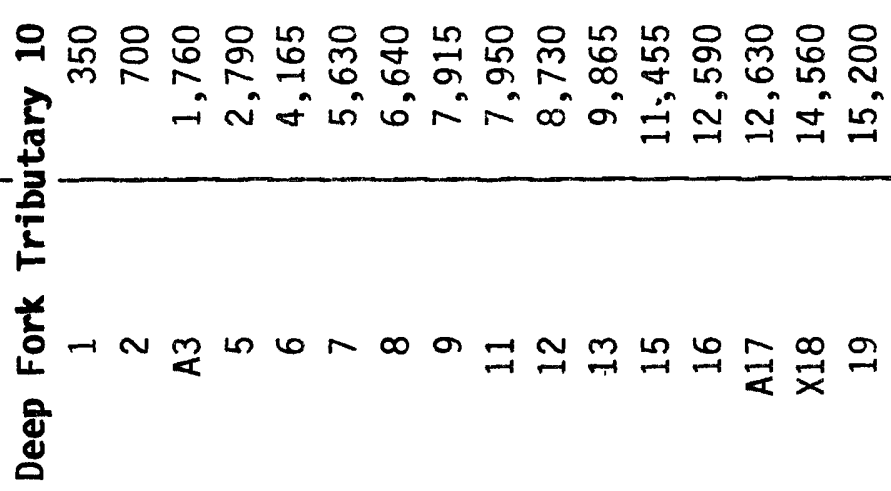 } & \multirow[t]{2}{*}{ 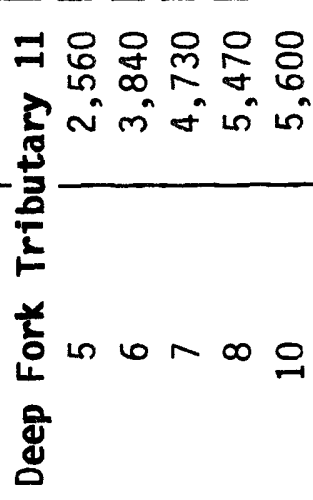 } \\
\hline & & 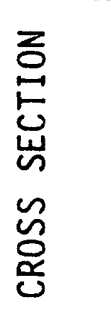 & & \\
\hline
\end{tabular}




\begin{tabular}{|c|c|c|c|c|}
\hline \multirow{3}{*}{ 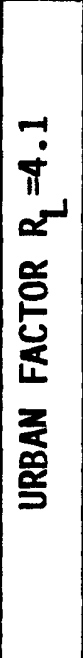 } & 愛 & 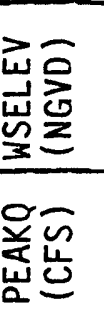 & 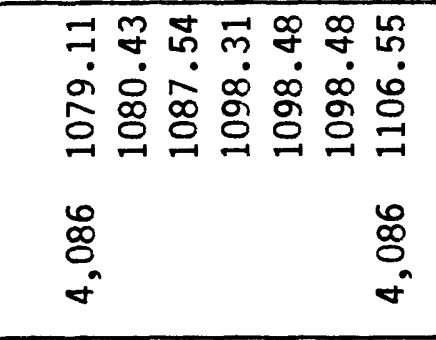 & 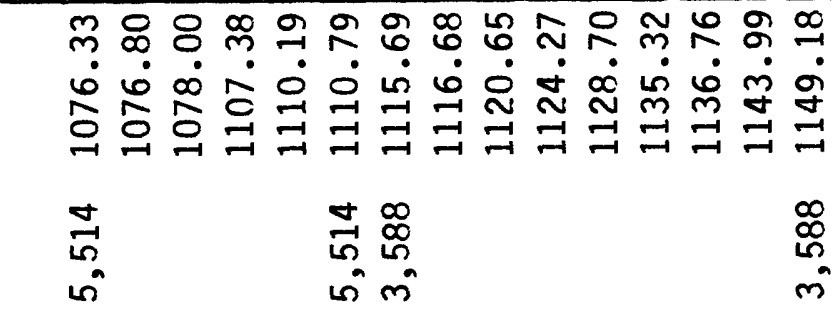 \\
\hline & \multirow{2}{*}{$\begin{array}{l}\frac{0}{5} \\
\frac{1}{1} \\
0\end{array}$} & 崖 & \multirow{2}{*}{ 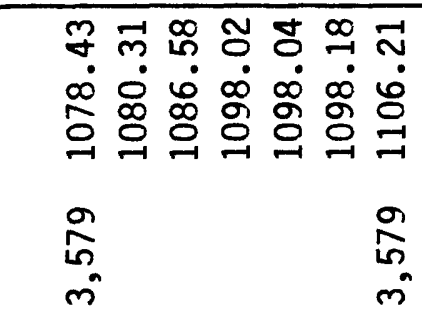 } & 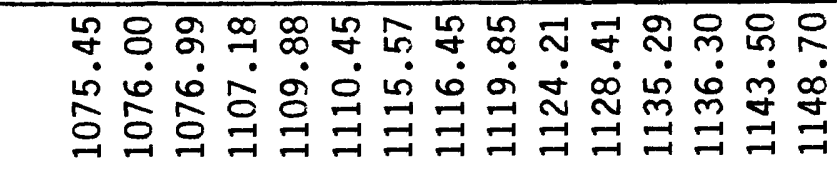 \\
\hline & & 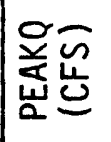 & & 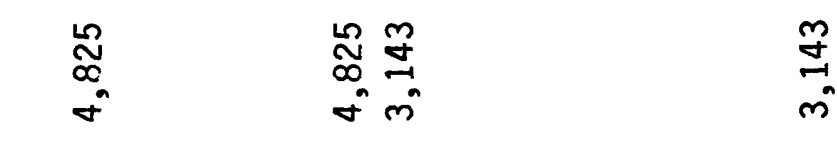 \\
\hline \multirow{8}{*}{ 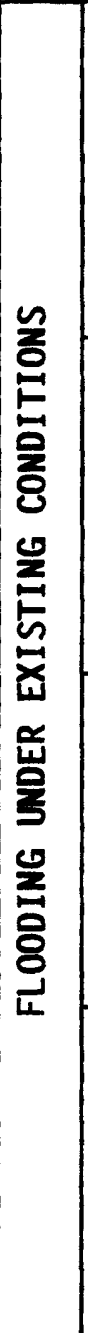 } & \multirow{2}{*}{$\begin{array}{l}\frac{0}{3} \\
\frac{1}{1} \\
0 \\
0\end{array}$} & 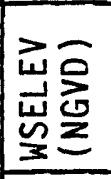 & \multirow{2}{*}{ 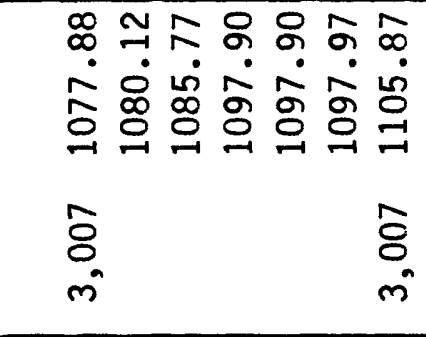 } & \multirow{2}{*}{ 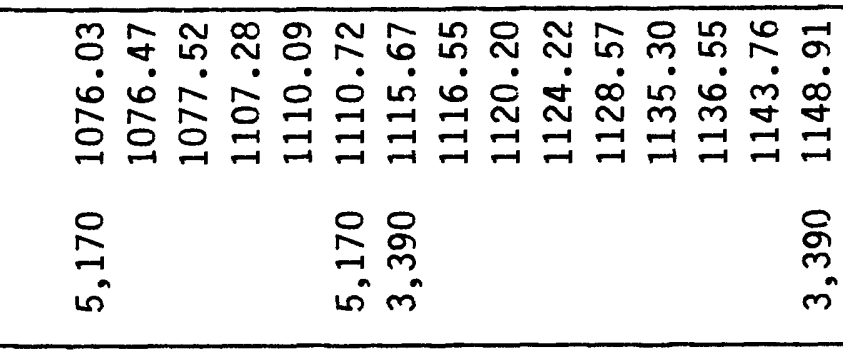 } \\
\hline & & 它芯 & & \\
\hline & \multirow{2}{*}{ 产 } & 峞塄 & \multirow{2}{*}{ 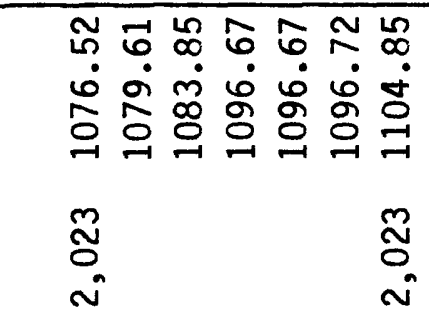 } & \multirow{2}{*}{ 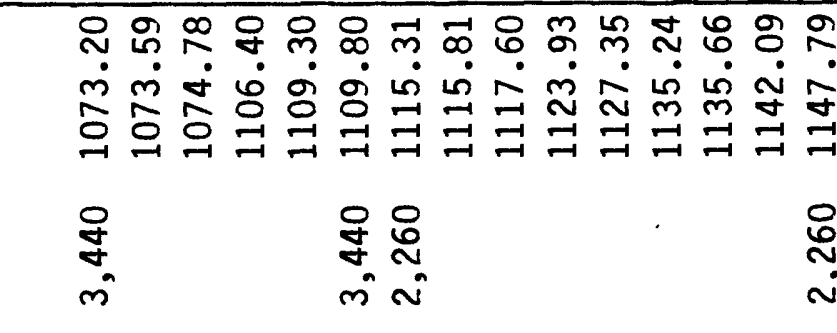 } \\
\hline & & $\begin{array}{l}\text { 음 } \\
\text { 岕苍 }\end{array}$ & & \\
\hline & \multirow{2}{*}{ 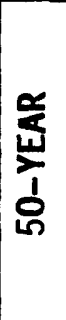 } & 崖 & \multirow{2}{*}{ 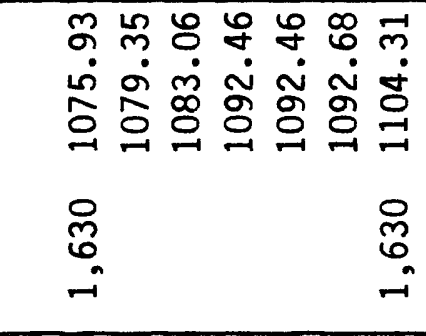 } & \multirow{2}{*}{ 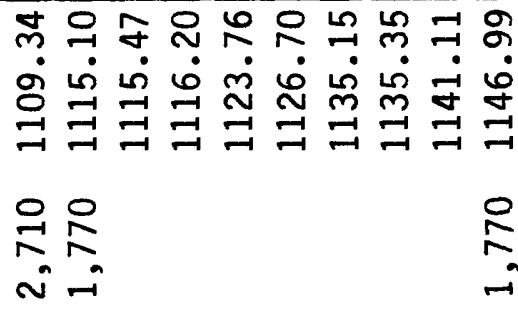 } \\
\hline & & 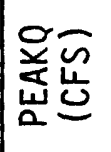 & & \\
\hline & \multirow{2}{*}{$\frac{\alpha}{\frac{1}{2}}$} & 峞 & \multirow{2}{*}{ 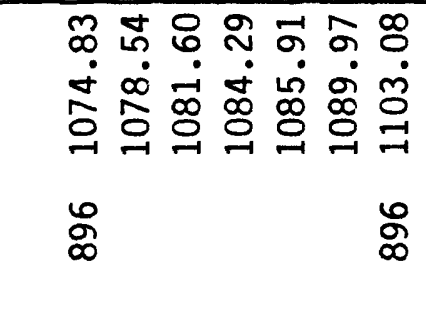 } & \multirow{2}{*}{ 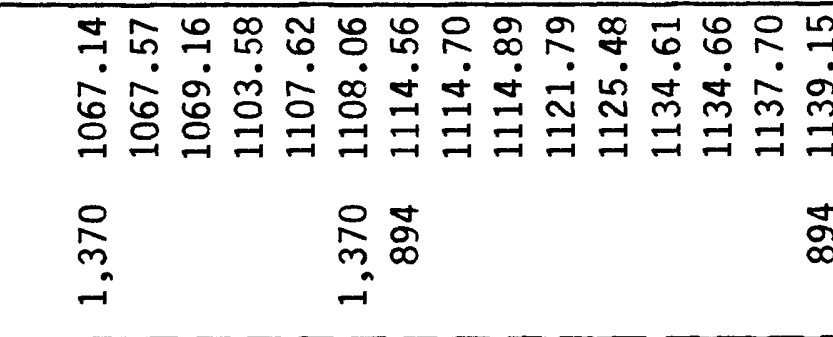 } \\
\hline & & 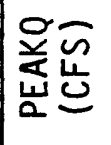 & & \\
\hline \multirow{2}{*}{ 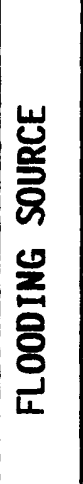 } & & 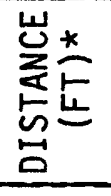 & \multirow{2}{*}{ 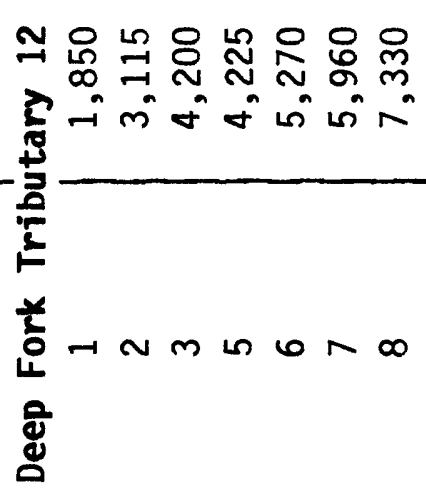 } & 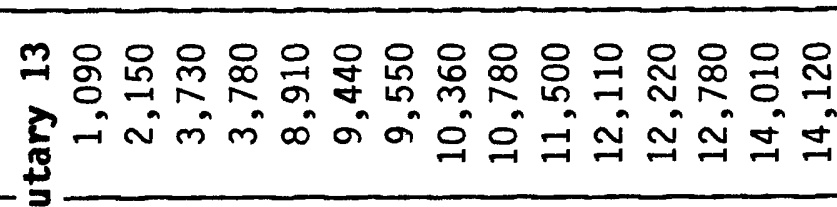 \\
\hline & & 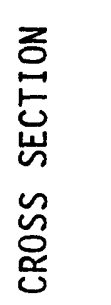 & & 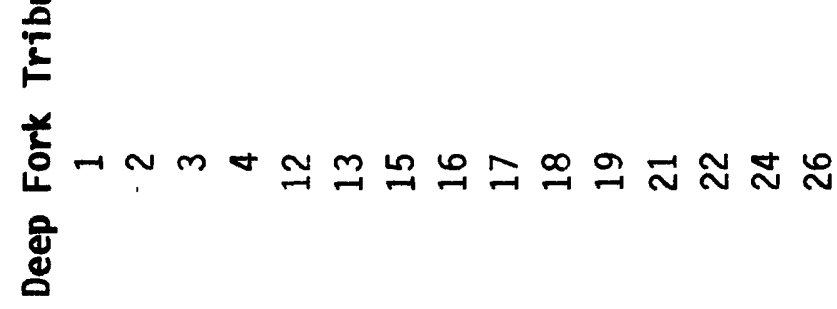 \\
\hline
\end{tabular}




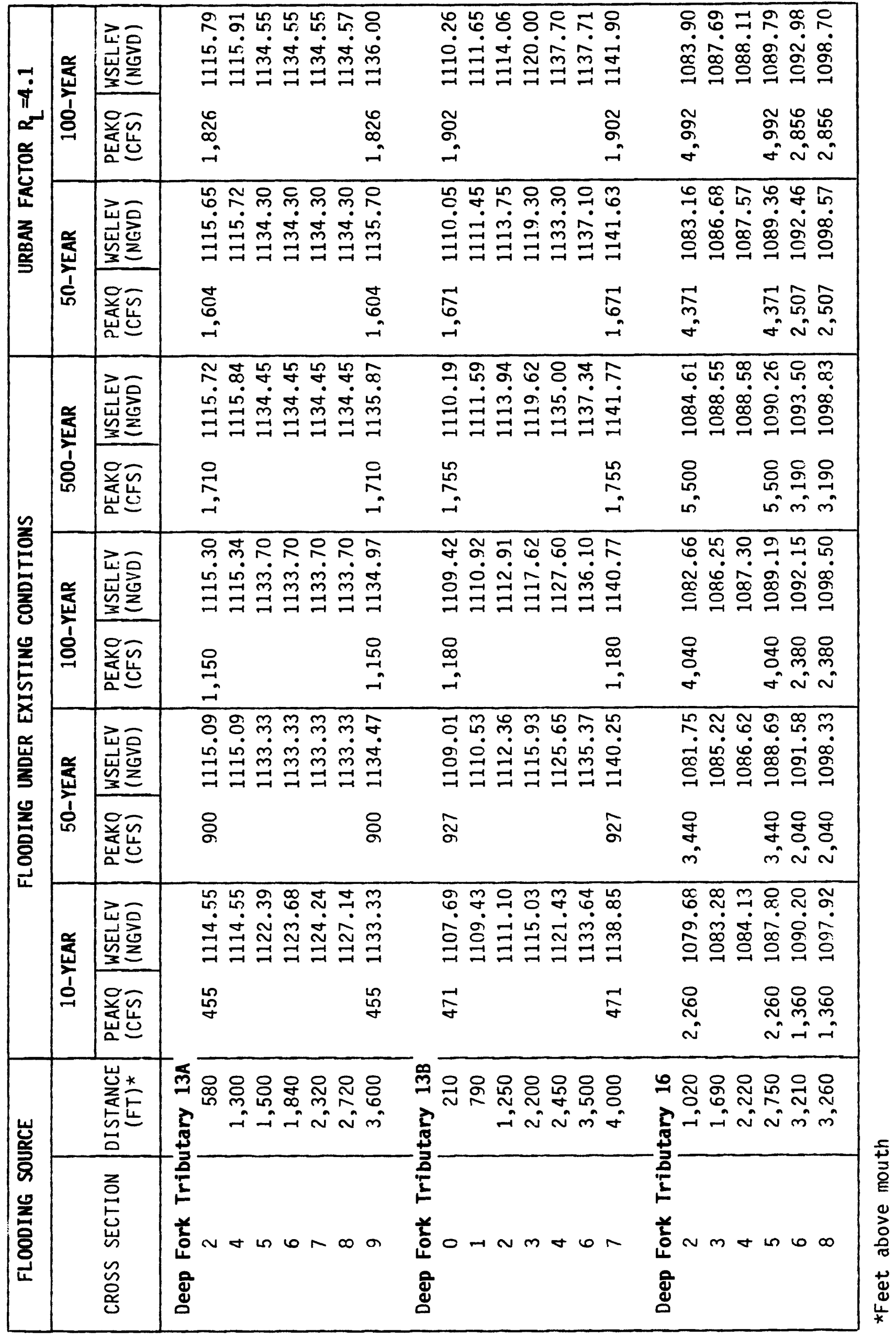




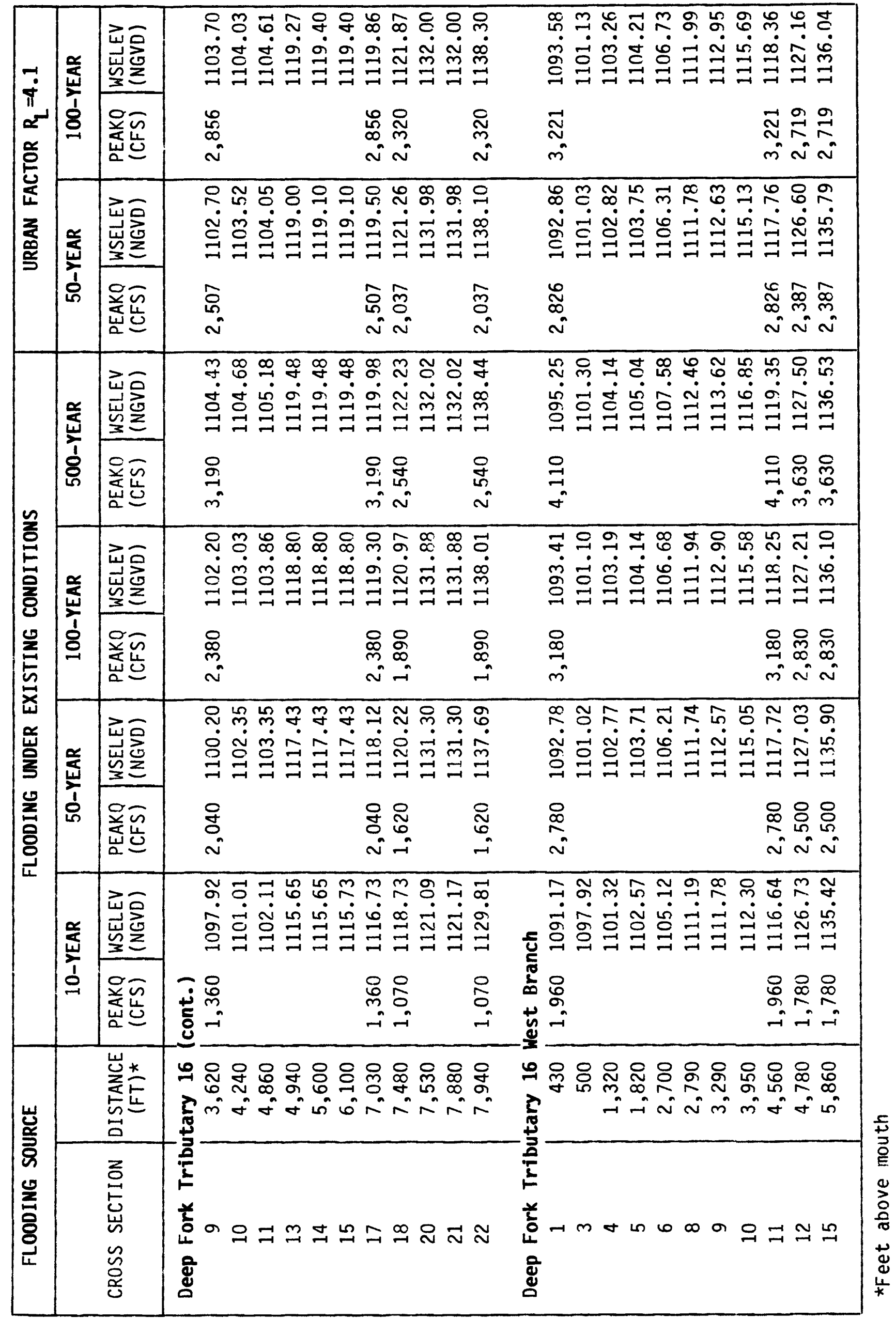




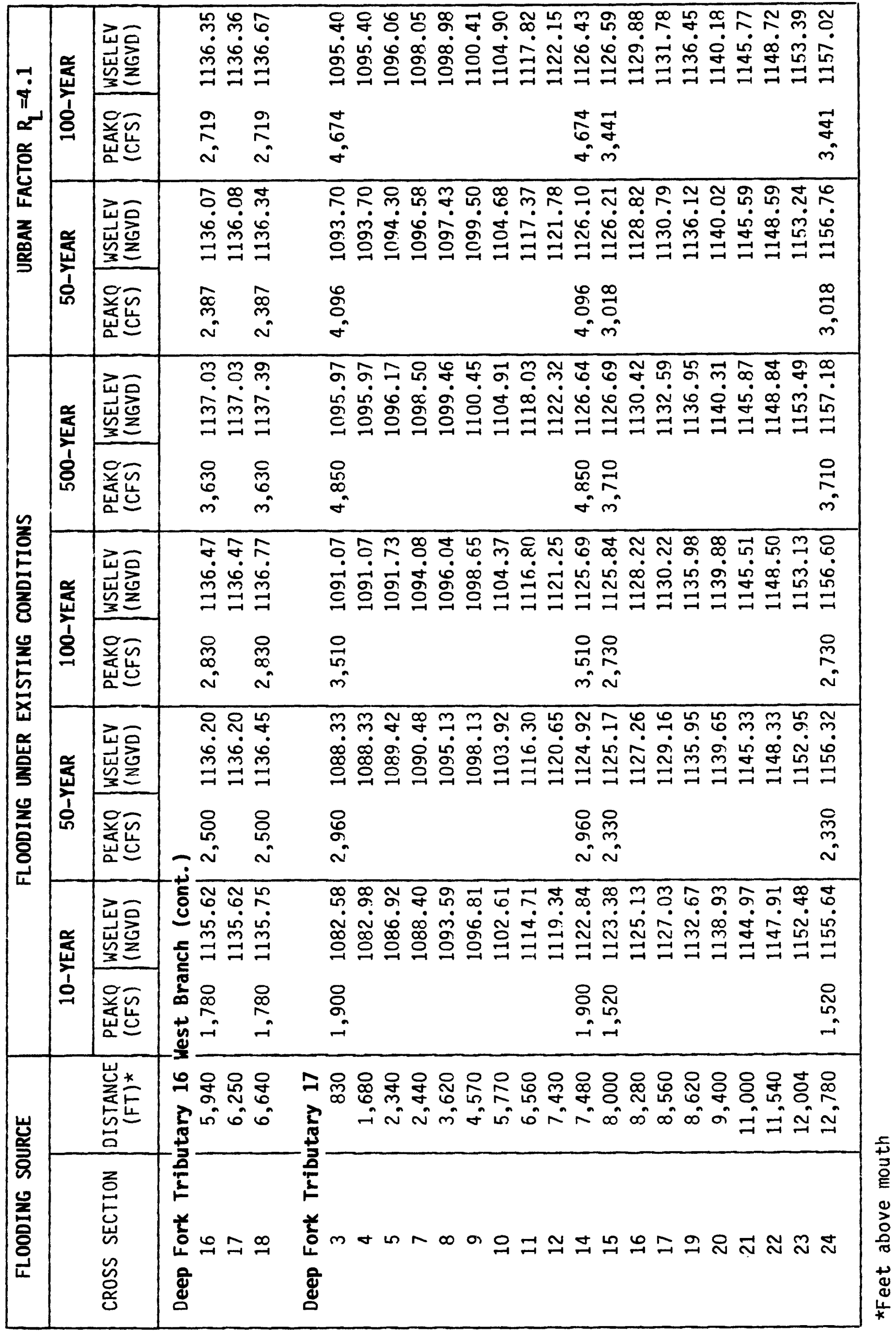




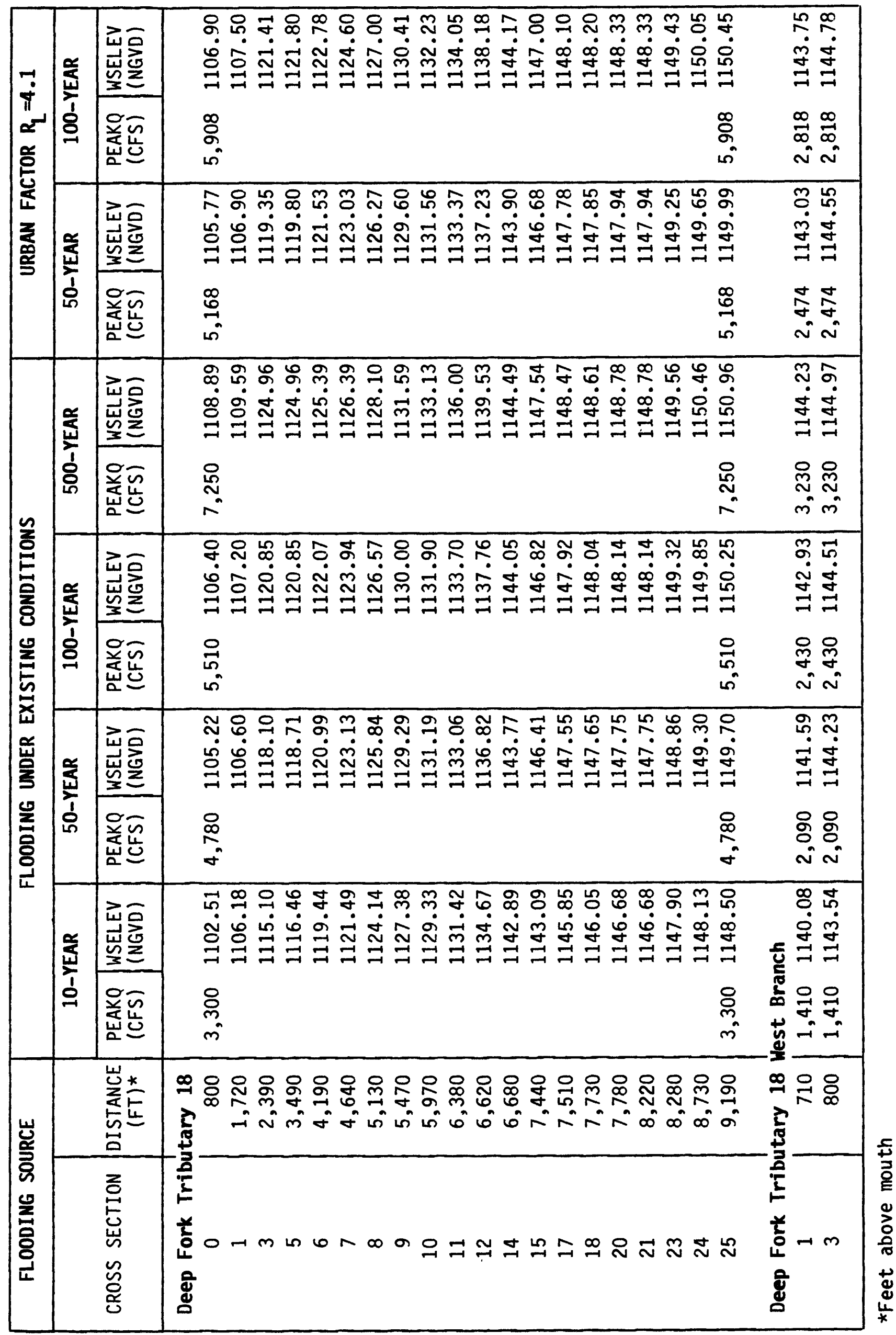




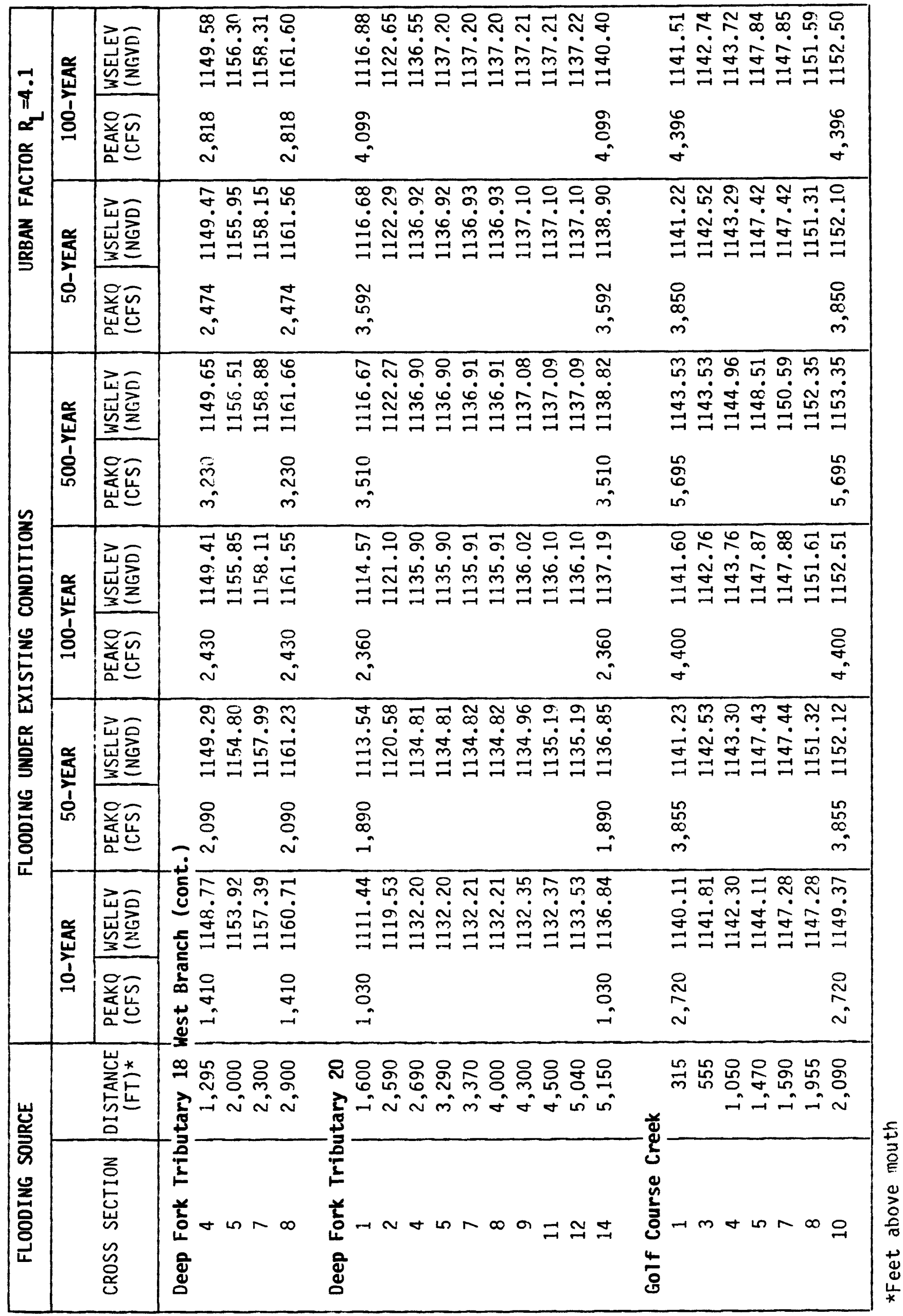




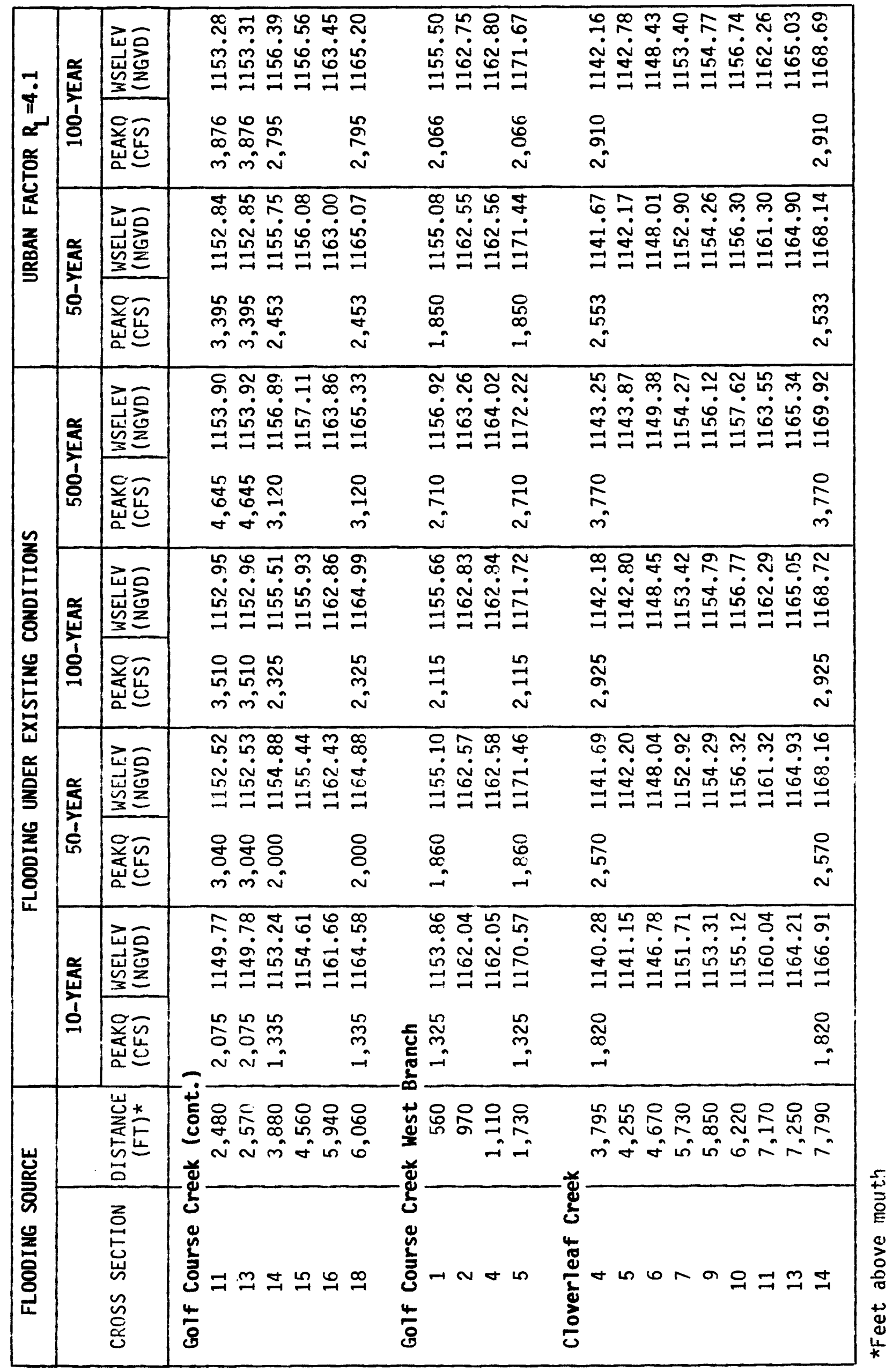




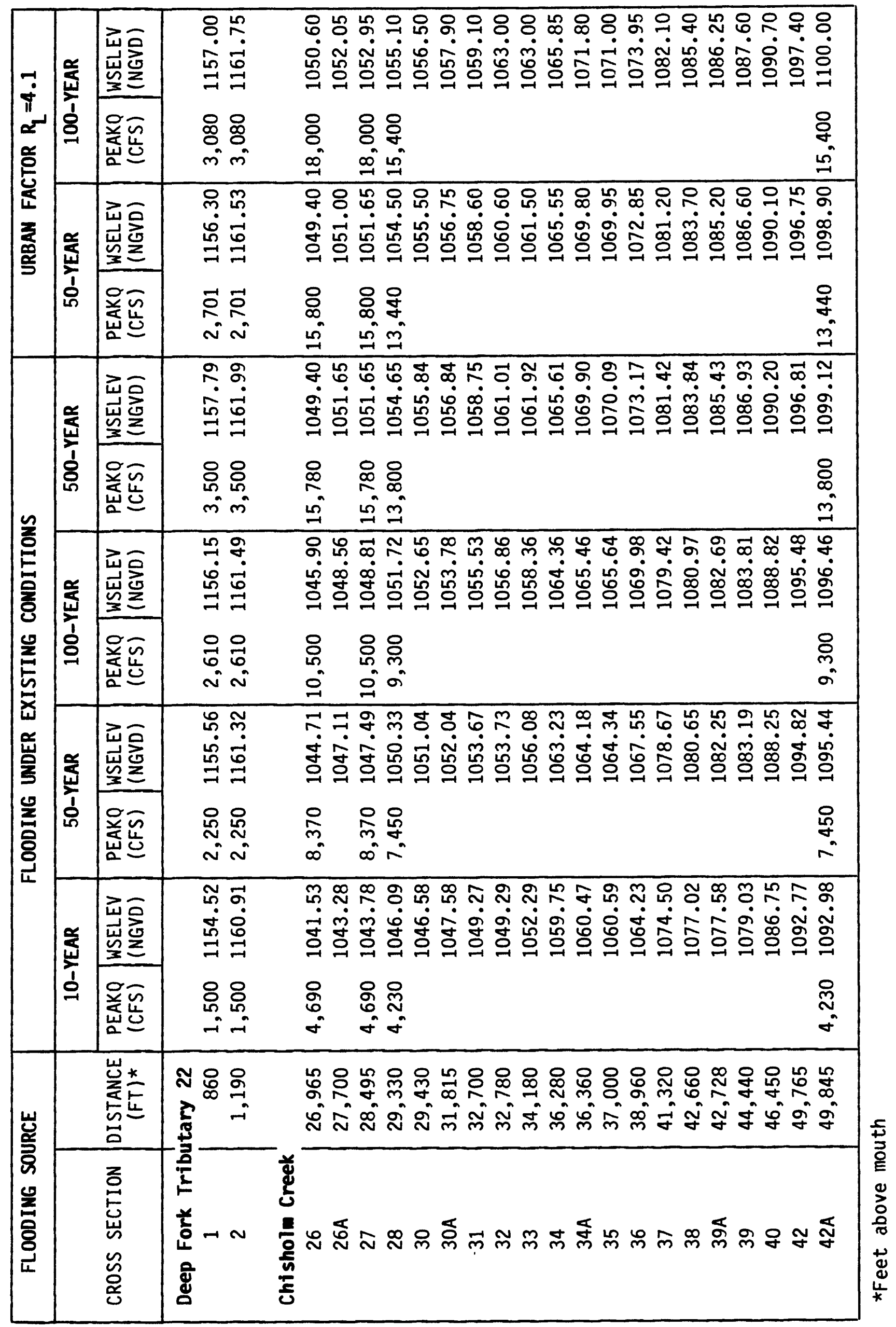




\begin{tabular}{|c|c|c|c|}
\hline \multirow{4}{*}{ 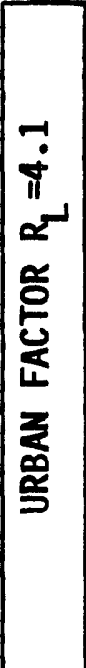 } & \multirow{2}{*}{ 咅 } & 岕怘 & 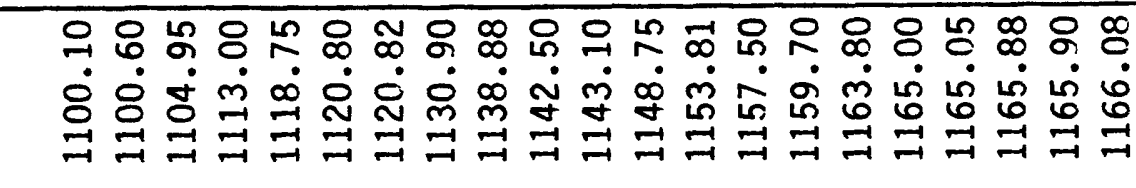 \\
\hline & & 总出 & 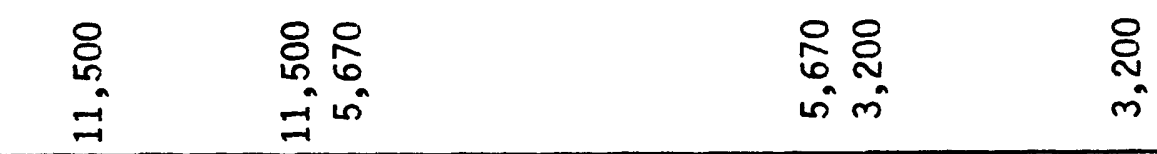 \\
\hline & \multirow{2}{*}{ 产 } & 崫总 & 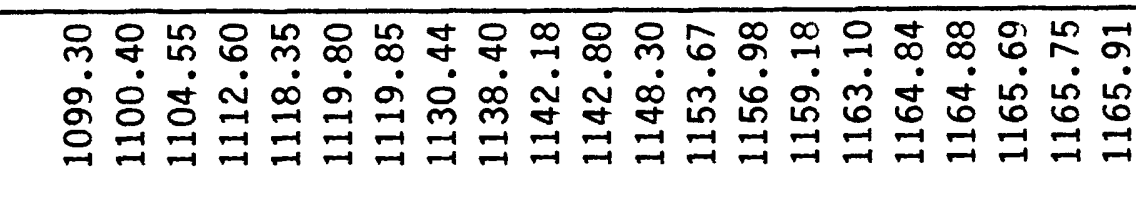 \\
\hline & & 尽岕 & 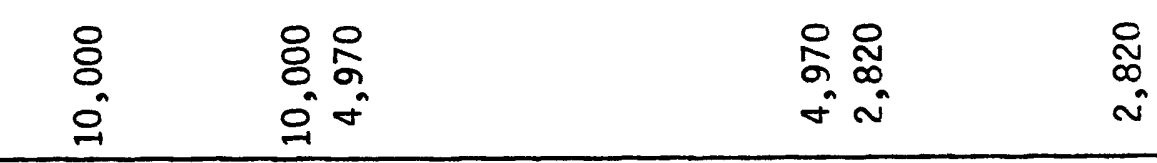 \\
\hline \multirow{8}{*}{ 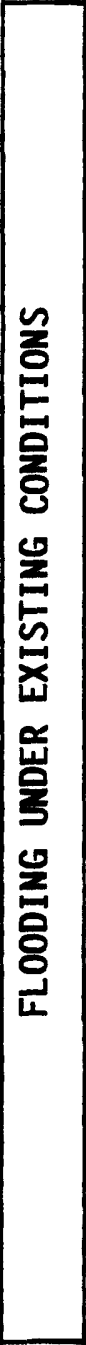 } & \multirow{2}{*}{ 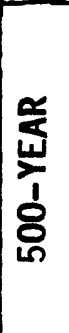 } & 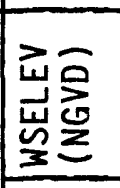 & 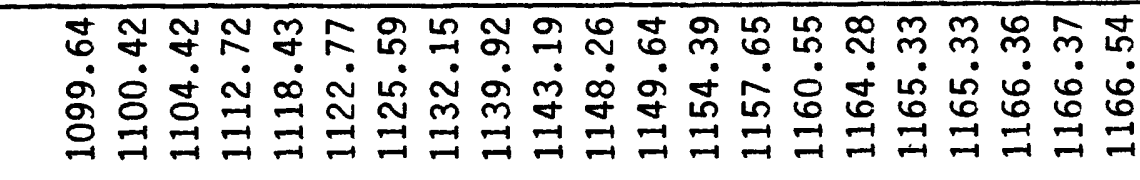 \\
\hline & & 莺心 & 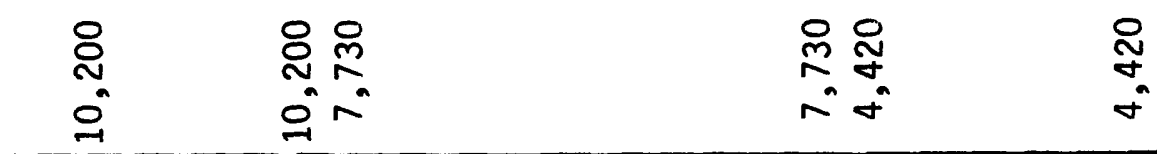 \\
\hline & \multirow{2}{*}{ 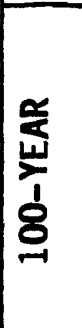 } & 岕高 & 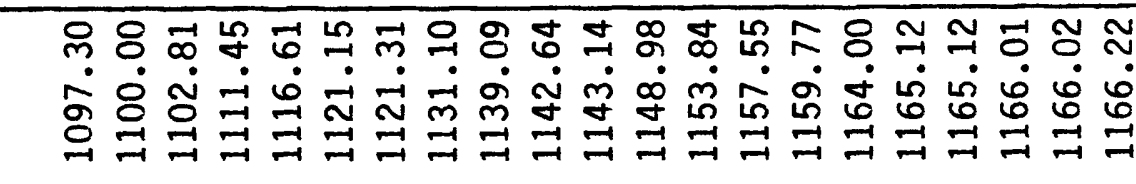 \\
\hline & & 通出 & $\begin{array}{ll}0 & 0 \\
\infty & \infty \\
0 & 0 \\
0 & 0 \\
0 & 0\end{array}$ \\
\hline & \multirow{2}{*}{ 妥 } & 岂㤩 & 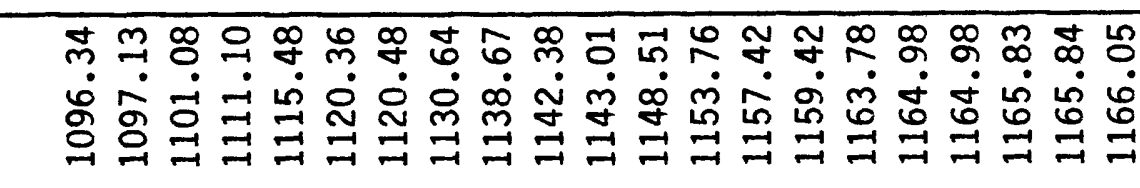 \\
\hline & & 通出 & $\begin{array}{cc}\substack{0 \\
m} \\
\text { in } \\
\text { in }\end{array}$ \\
\hline & \multirow{2}{*}{$\underset{\frac{1}{4}}{\frac{\alpha}{\grave{c}}}$} & 山己 & 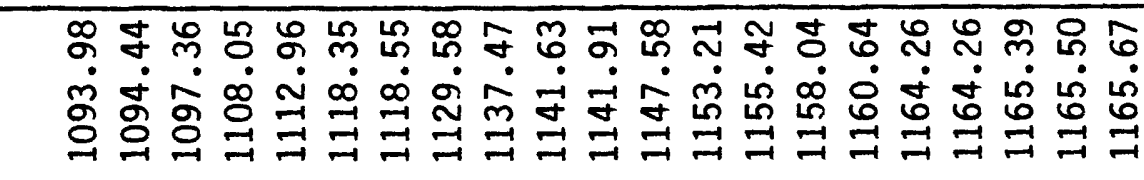 \\
\hline & & 通出 & 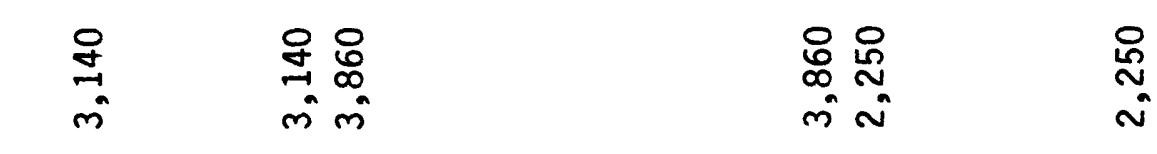 \\
\hline \multirow{2}{*}{ 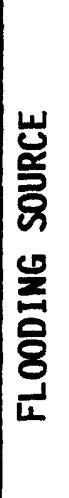 } & & 岕岕 & 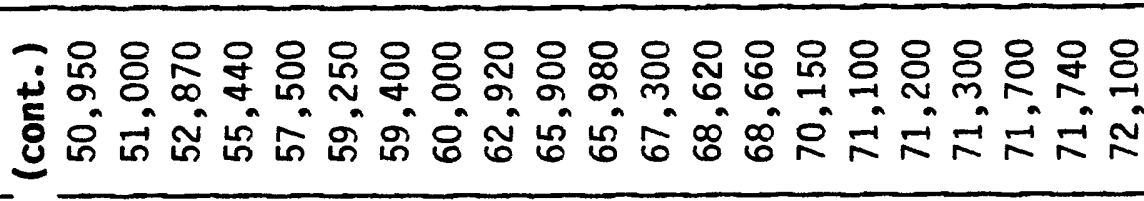 \\
\hline & & 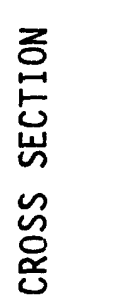 & 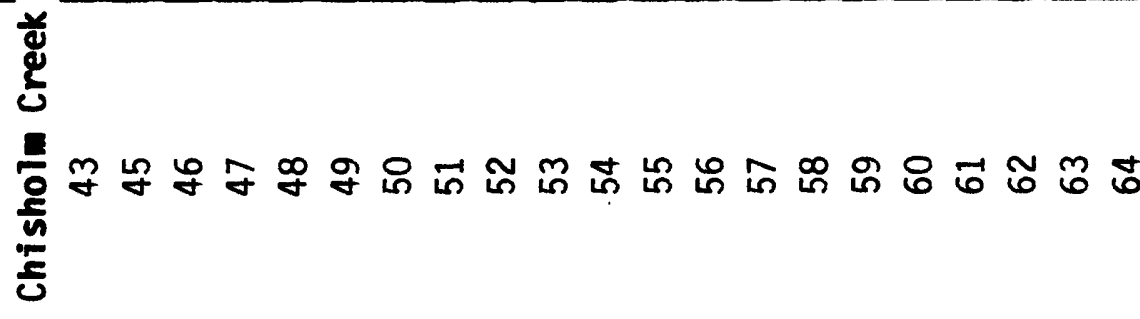 \\
\hline
\end{tabular}




\begin{tabular}{|c|c|c|c|c|c|c|}
\hline \multirow{3}{*}{ 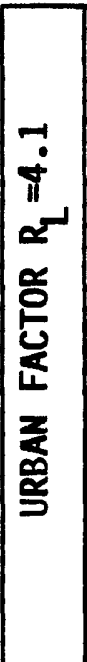 } & 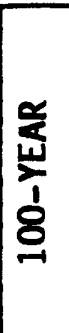 & 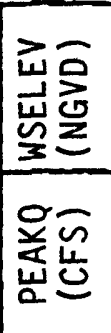 & 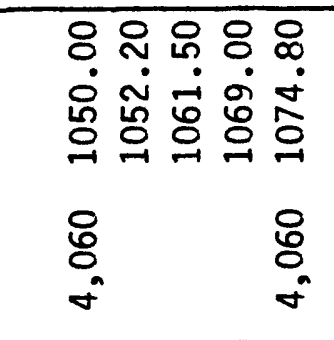 & 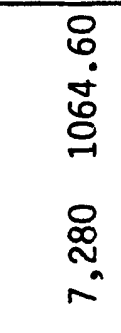 & 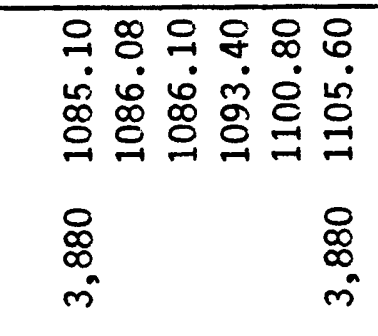 & 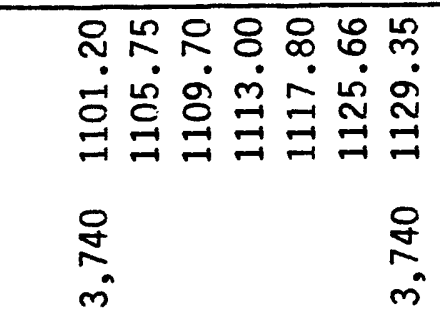 \\
\hline & \multirow{2}{*}{$\frac{\frac{0}{5}}{\frac{1}{1}}$} & 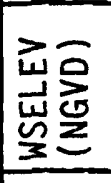 & 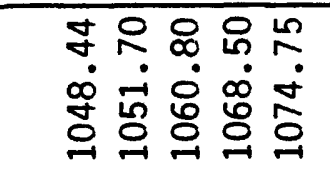 & $\begin{array}{l}\dot{0} \\
\dot{0} \\
\dot{0}\end{array}$ & 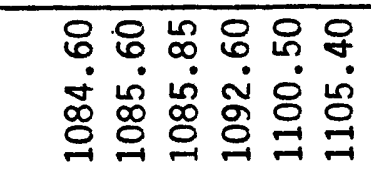 & $\begin{array}{l}0 \\
\infty \\
\infty\end{array}$ \\
\hline & & 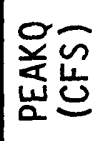 & $\begin{array}{ll}0 & 0 \\
i n & n \\
m & m\end{array}$ & $\frac{0}{n}$ & 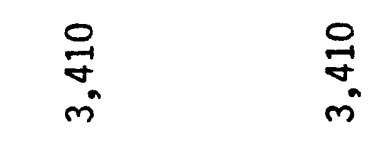 & 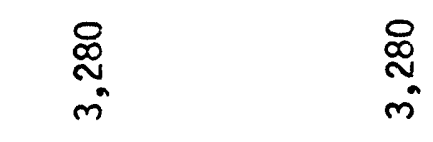 \\
\hline \multirow{8}{*}{ 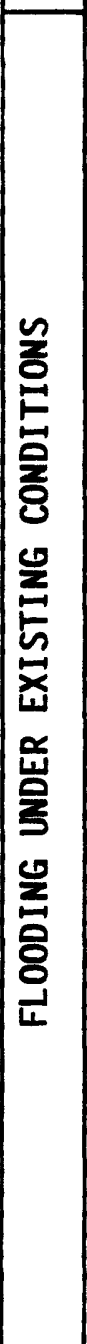 } & \multirow{2}{*}{ 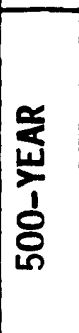 } & 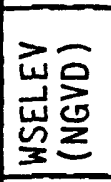 & 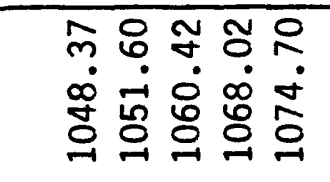 & $\underset{\sim}{\dddot{\sim}}$ & 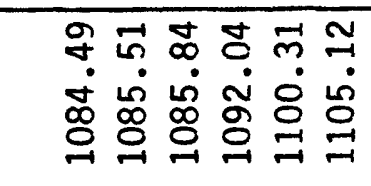 & 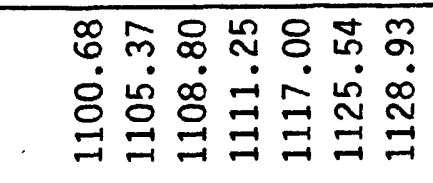 \\
\hline & & 通 & $\begin{array}{ll}\frac{0}{m} & \frac{O}{m} \\
m & m\end{array}$ & $\frac{8}{6}$ & $\begin{array}{ll}\text { N } & \text { N } \\
m & m\end{array}$ & 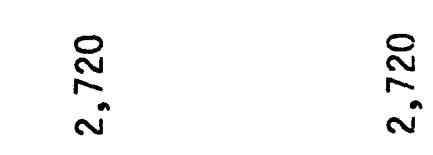 \\
\hline & \multirow{2}{*}{ 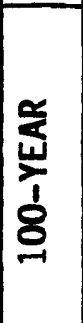 } & 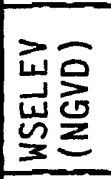 & 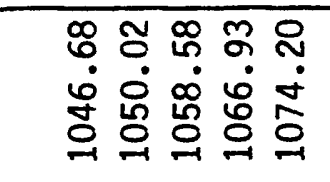 & $\begin{array}{l}0 \\
\dot{0} \\
\dot{0}\end{array}$ & 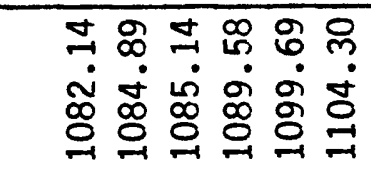 & 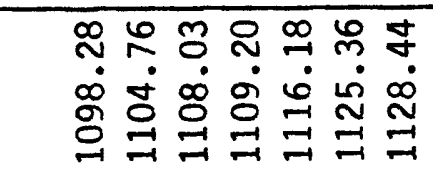 \\
\hline & & 产 & 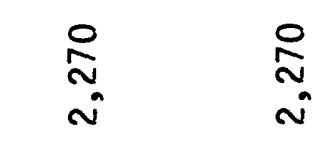 & 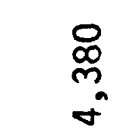 & $\begin{array}{ll}0 & 8 \\
\sim & n\end{array}$ & 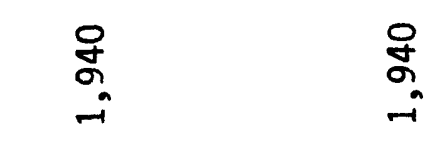 \\
\hline & \multirow{2}{*}{ 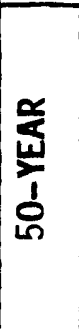 } & 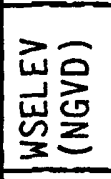 & 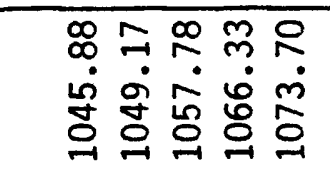 & б̆ & 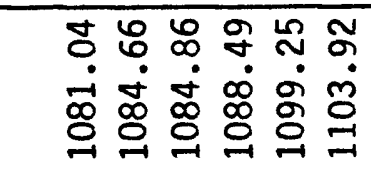 & 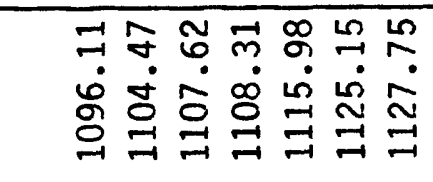 \\
\hline & & 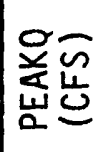 & $\begin{array}{ll}\circ & \stackrel{\circ}{\infty} \\
- & \infty\end{array}$ & 㝦 & $\stackrel{\text { 足 }}{\text { ri }}$ & 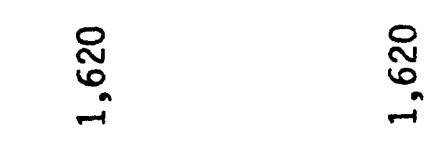 \\
\hline & \multirow{2}{*}{ 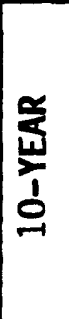 } & 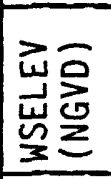 & 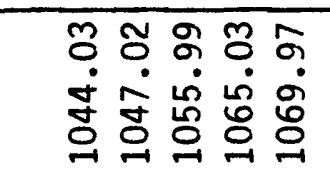 & 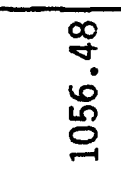 & 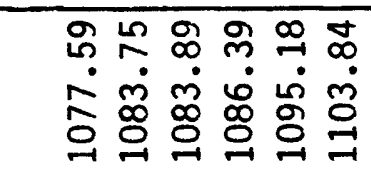 & 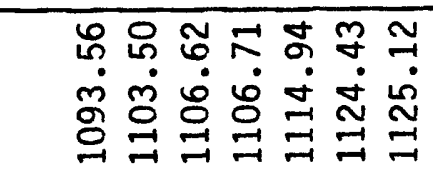 \\
\hline & & 通 & $m \stackrel{0}{0} \underset{0}{0} \quad \stackrel{0}{0}$ & $\begin{array}{r}8 \\
+ \text { n }\end{array}$ & $0^{\frac{0}{\sigma}}$ & \& \\
\hline \multirow{2}{*}{ 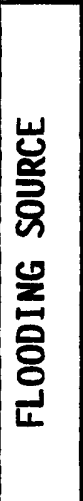 } & & 岕莣芯 & 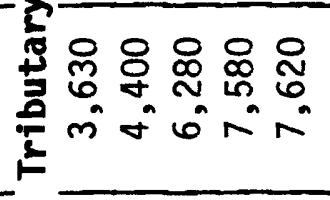 & 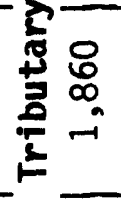 & 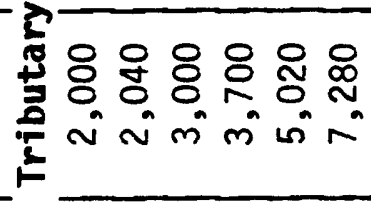 & 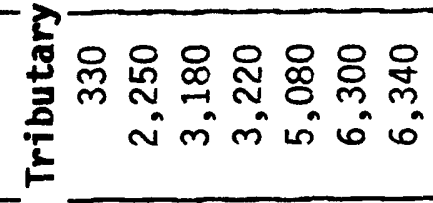 \\
\hline & & 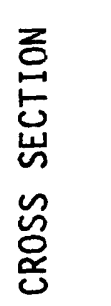 & 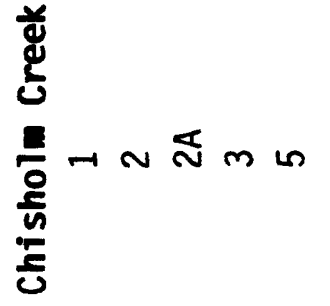 & $\begin{array}{l}\frac{0}{0} \\
\frac{0}{0} \\
\frac{0}{0} \\
\frac{n}{5}\end{array}$ & $\begin{array}{l}\frac{y}{d} \\
\frac{d}{U} \\
\frac{E}{0}-\sim m m * n \\
\frac{0}{n} \\
\frac{n}{U}\end{array}$ & $\begin{array}{l}\frac{1}{d} \\
\frac{d}{U} \\
\frac{E}{\frac{D}{n}} \\
\frac{E}{U}\end{array}$ \\
\hline
\end{tabular}




\begin{tabular}{|c|c|c|c|c|}
\hline \multirow{3}{*}{ 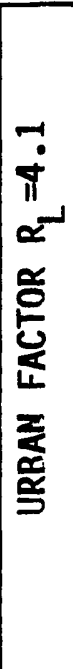 } & 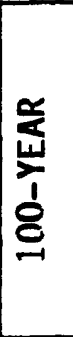 & 峞完 & 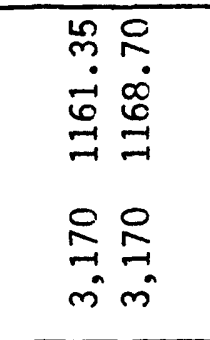 & 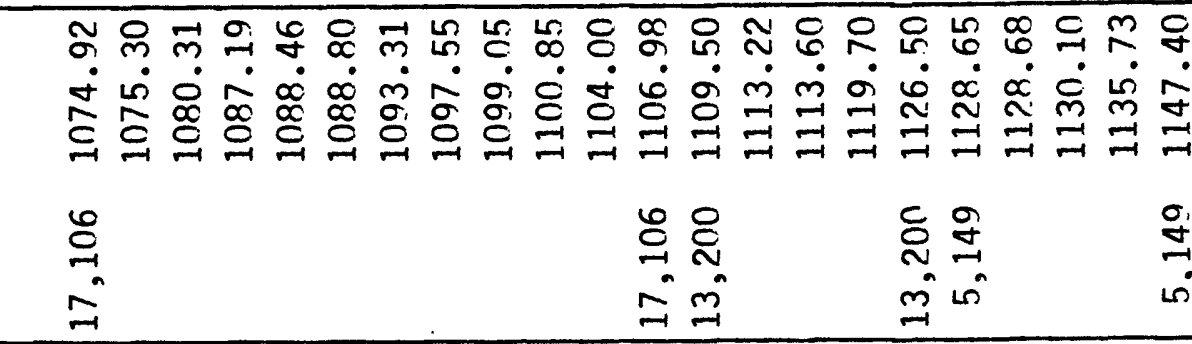 \\
\hline & \multirow{2}{*}{ 旁 } & 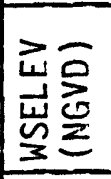 & 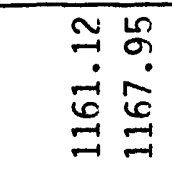 & 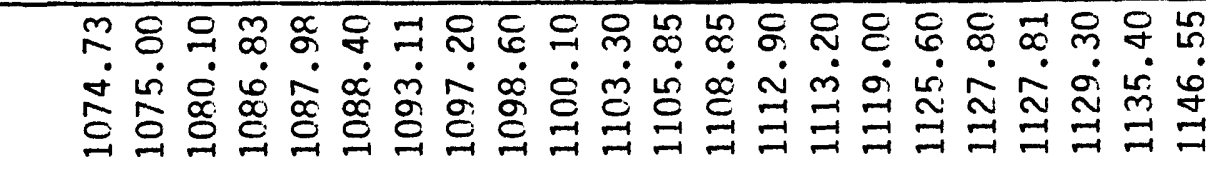 \\
\hline & & 旁苍 & 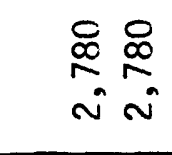 & 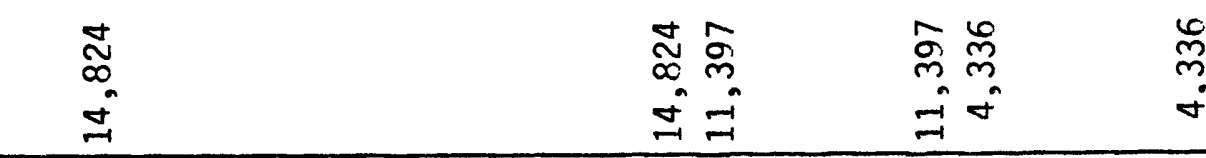 \\
\hline \multirow{8}{*}{ 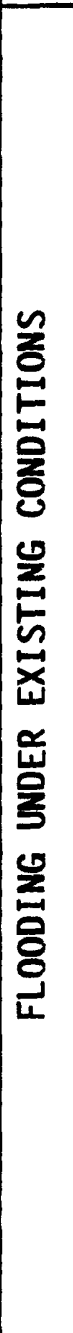 } & \multirow{2}{*}{ 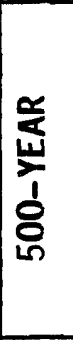 } & 峞员 & 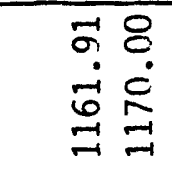 & 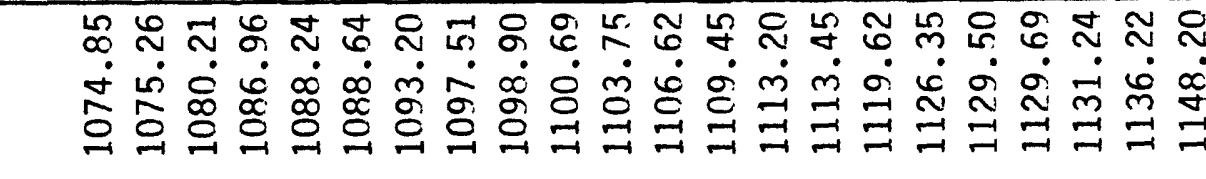 \\
\hline & & 美芯 & $\begin{array}{l}\text { \& } \\
\text { g } \\
\dot{q} \text { m } \\
m\end{array}$ & 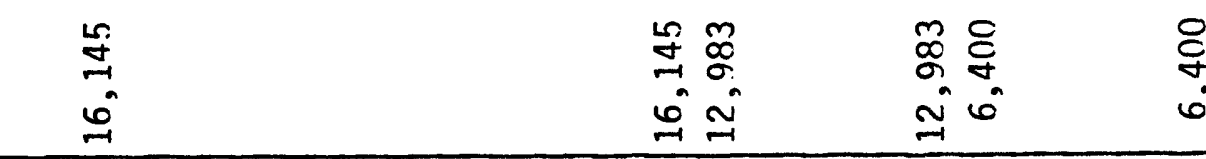 \\
\hline & \multirow{2}{*}{ 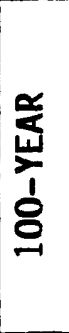 } & 峞市 & 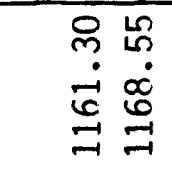 & 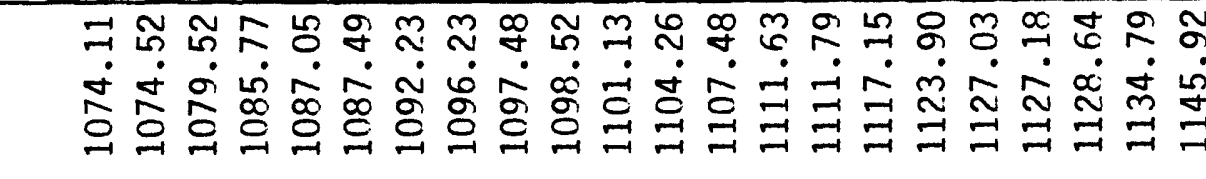 \\
\hline & & 恙芯 & 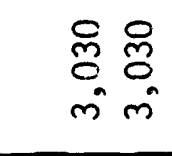 & 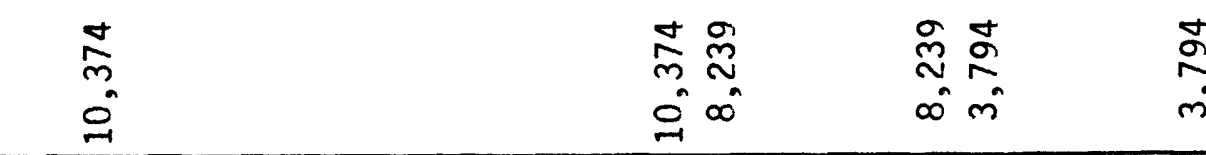 \\
\hline & \multirow{2}{*}{ 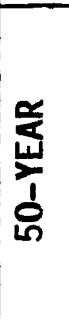 } & 崖员 & 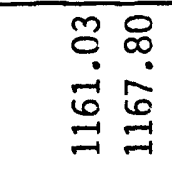 & 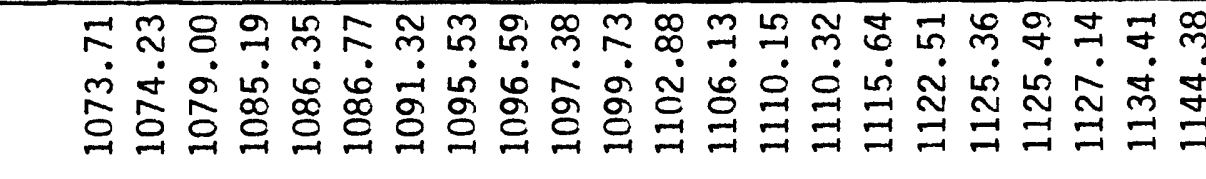 \\
\hline & & 道出 & 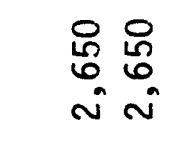 & 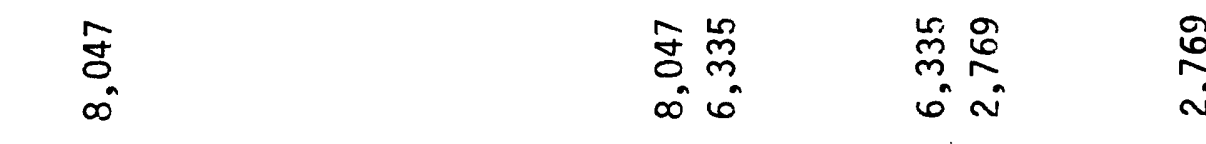 \\
\hline & \multirow{2}{*}{ 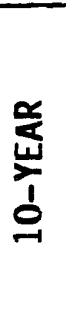 } & 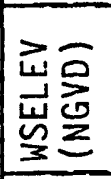 & \begin{tabular}{ll}
2 & 8 \\
0 & 0 \\
0 & 0 \\
\hdashline & 0 \\
$=$ & $=1$
\end{tabular} & 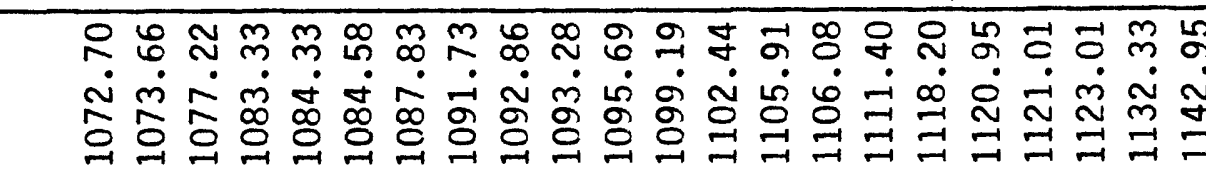 \\
\hline & & 总芯 & 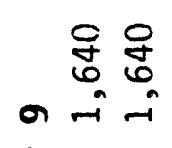 & 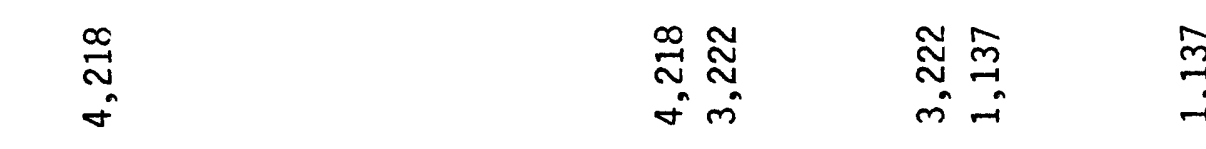 \\
\hline \multirow{2}{*}{ 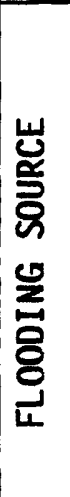 } & & 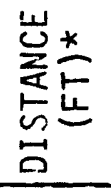 & $\sum_{\substack{3 \\
0}}^{8} 8$ & 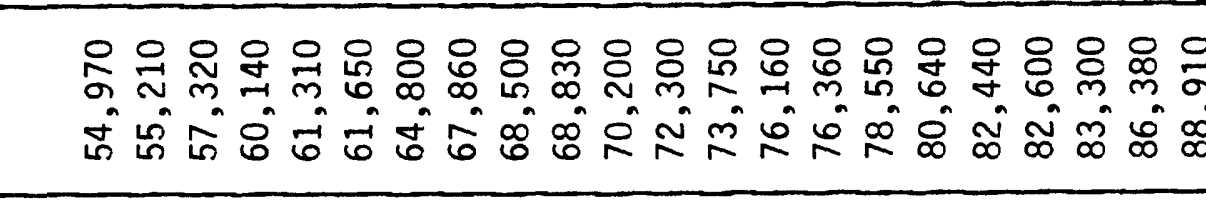 \\
\hline & & 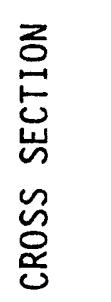 & 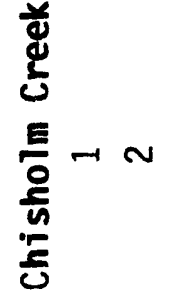 & 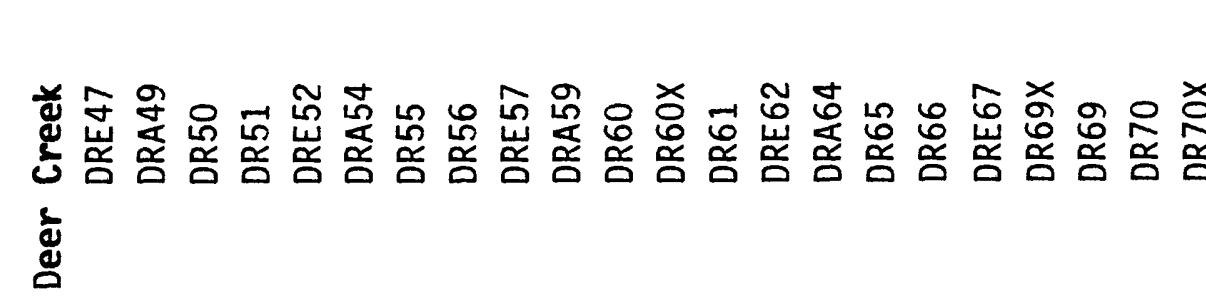 \\
\hline
\end{tabular}




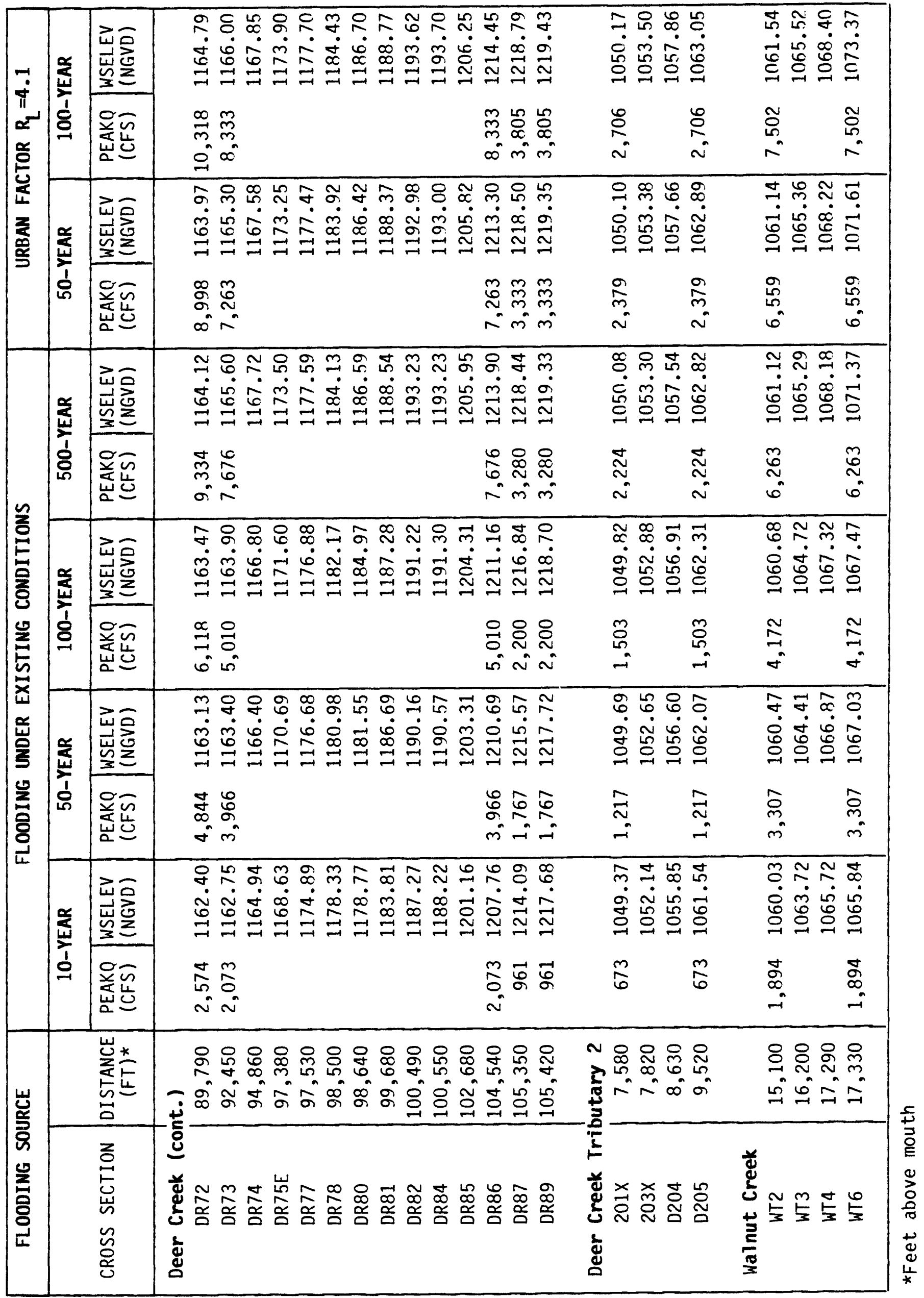




\begin{tabular}{|c|c|c|c|}
\hline \multirow{4}{*}{ 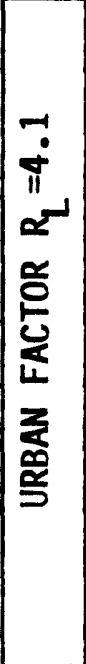 } & \multirow{2}{*}{$\frac{\alpha}{\frac{\alpha}{5}}$} & 岕㤩 & 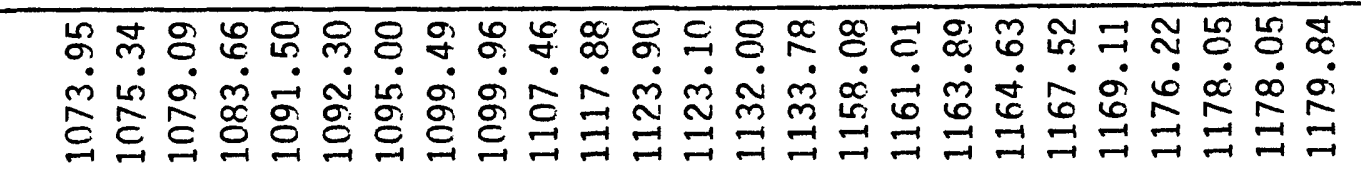 \\
\hline & & 㱯出 & 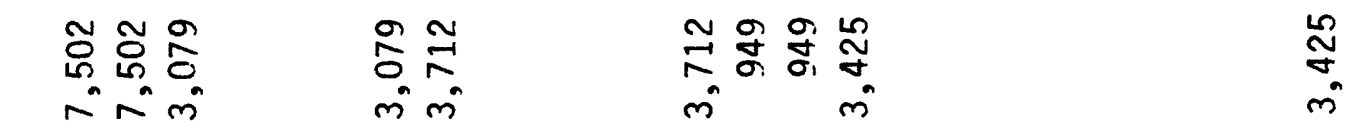 \\
\hline & \multirow{2}{*}{ 愛 } & 崖 & 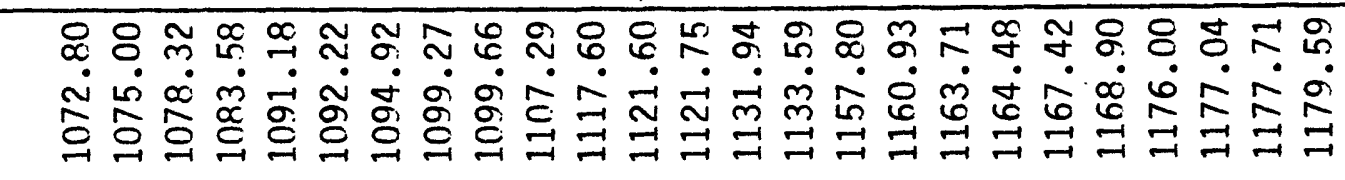 \\
\hline & & 递苍 & 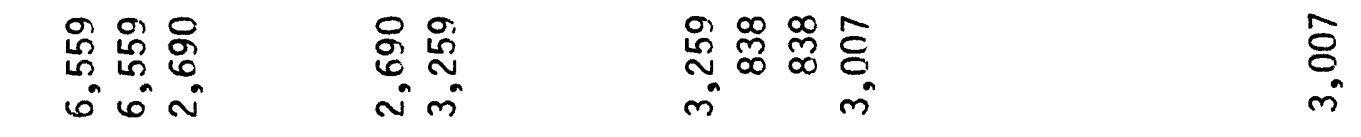 \\
\hline \multirow{8}{*}{ 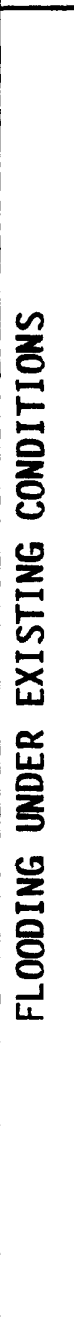 } & \multirow{2}{*}{ 莣 } & 岂总 & 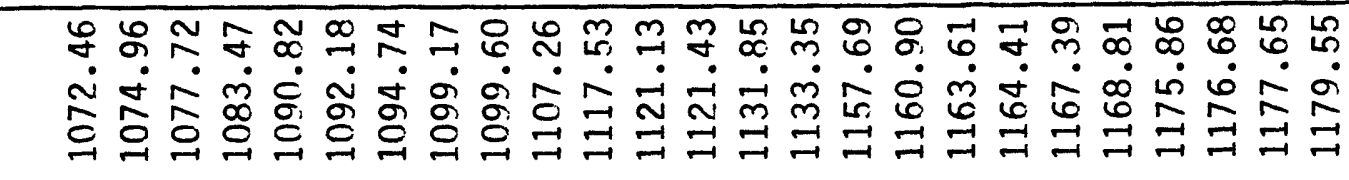 \\
\hline & & 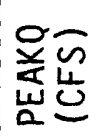 & 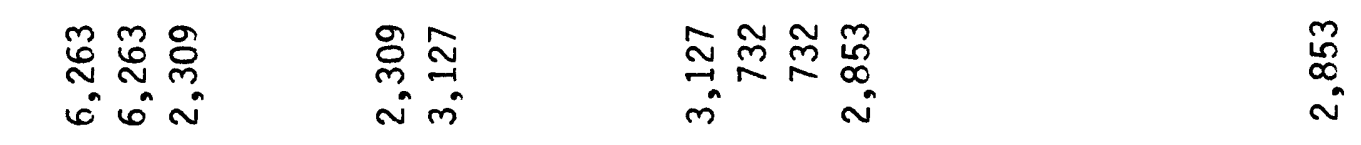 \\
\hline & \multirow{2}{*}{ 紊 } & 岂离 & 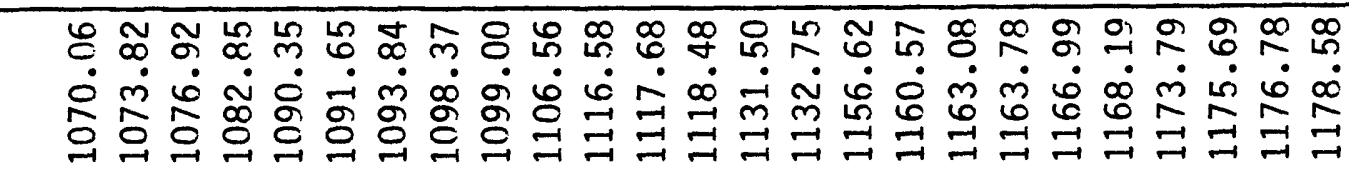 \\
\hline & & 递苍 & 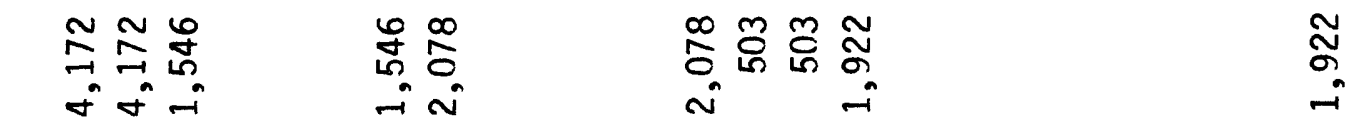 \\
\hline & \multirow{2}{*}{ 皆 } & 岂㤩 & 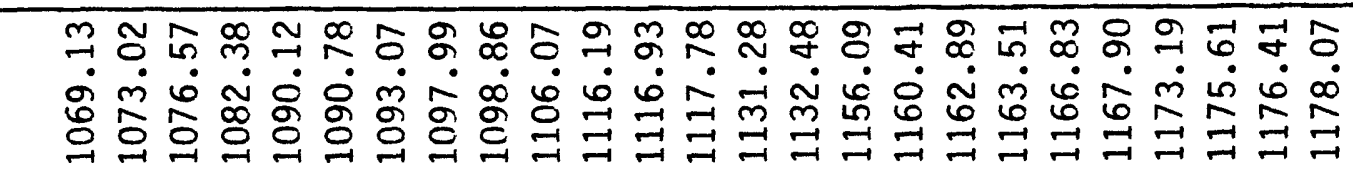 \\
\hline & & 递出 & 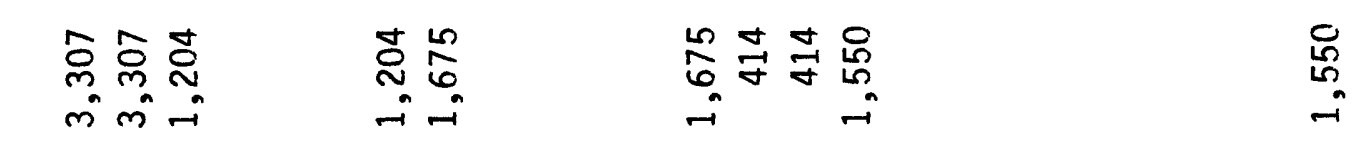 \\
\hline & \multirow{2}{*}{ 产 } & 岂量 & \multirow{2}{*}{ 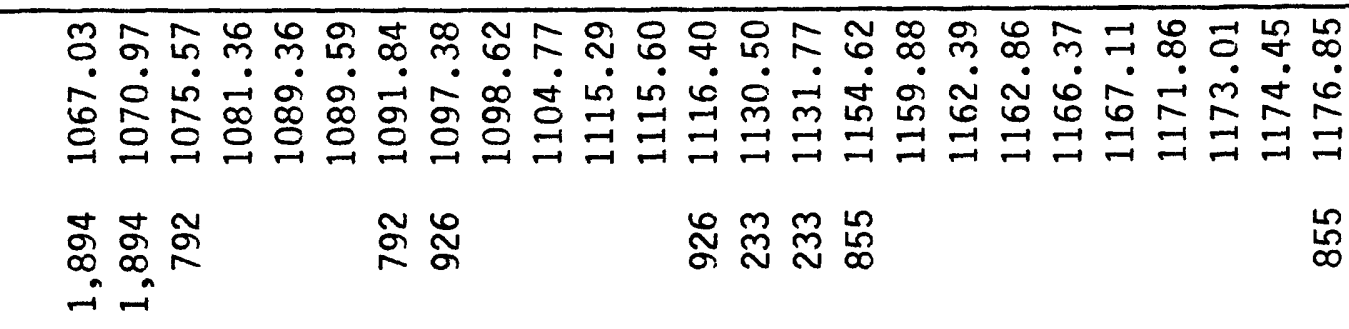 } \\
\hline & & 序芯 & \\
\hline \multirow{2}{*}{ 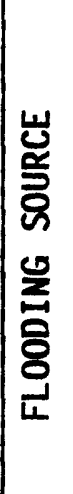 } & & 岁岕 & 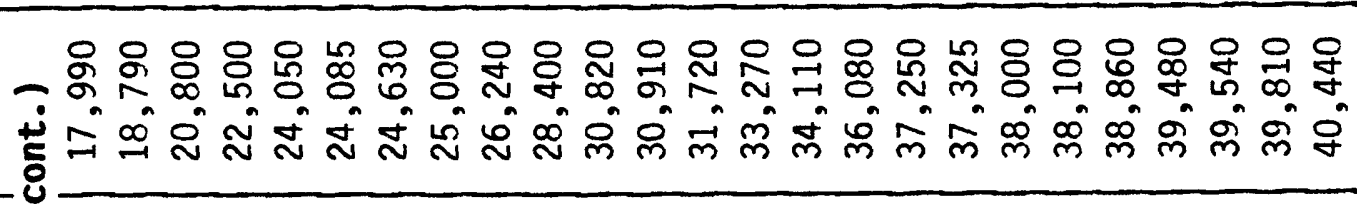 \\
\hline & & 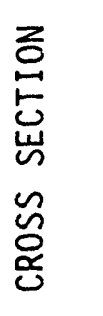 & 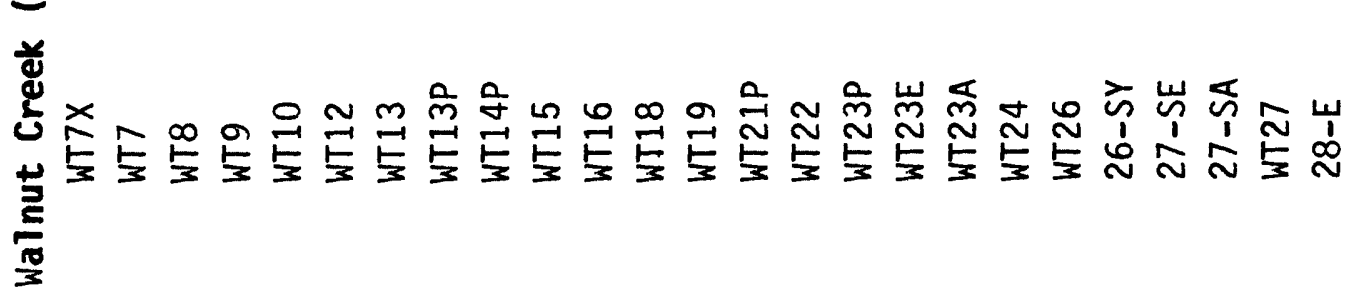 \\
\hline
\end{tabular}




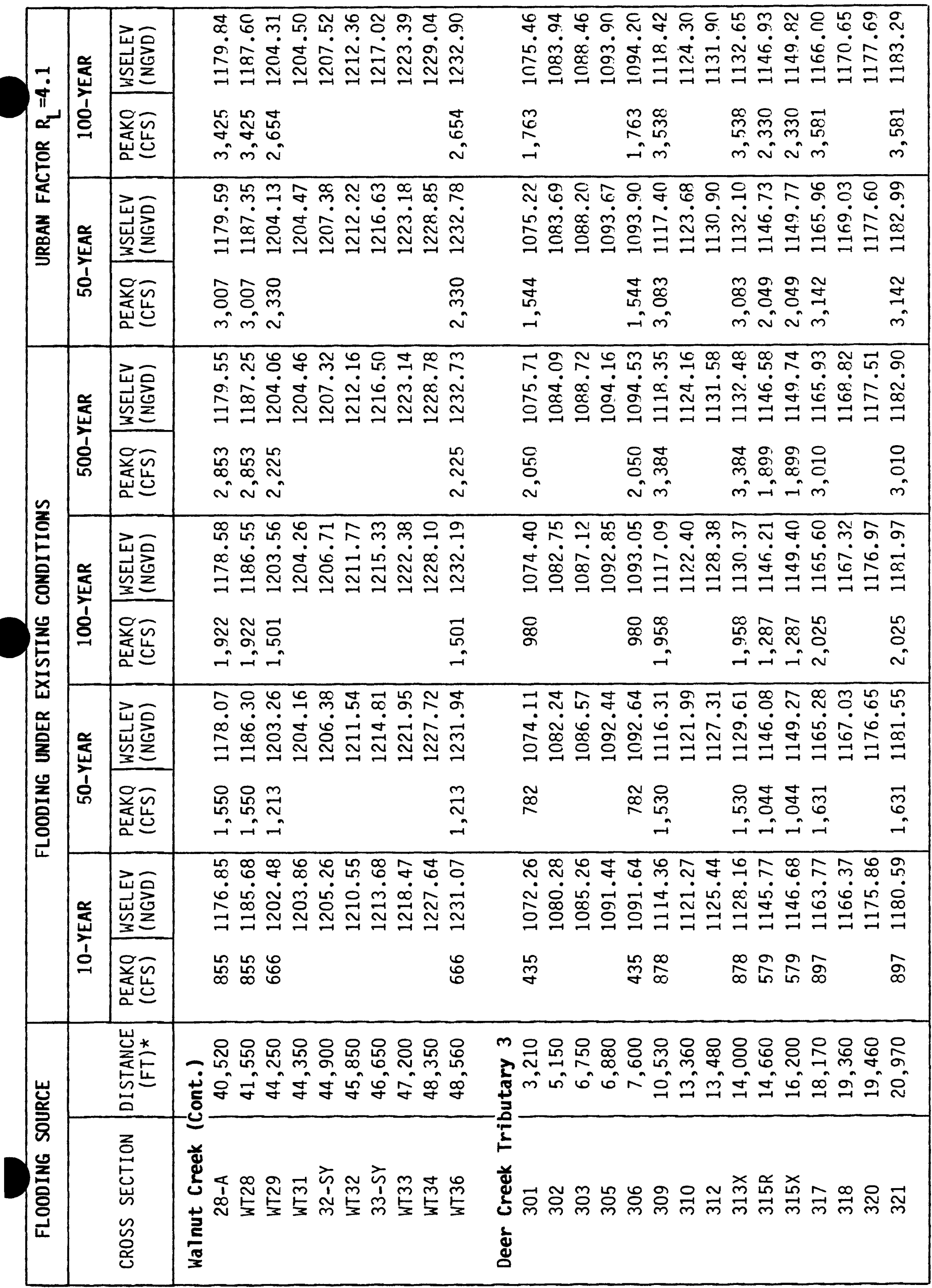




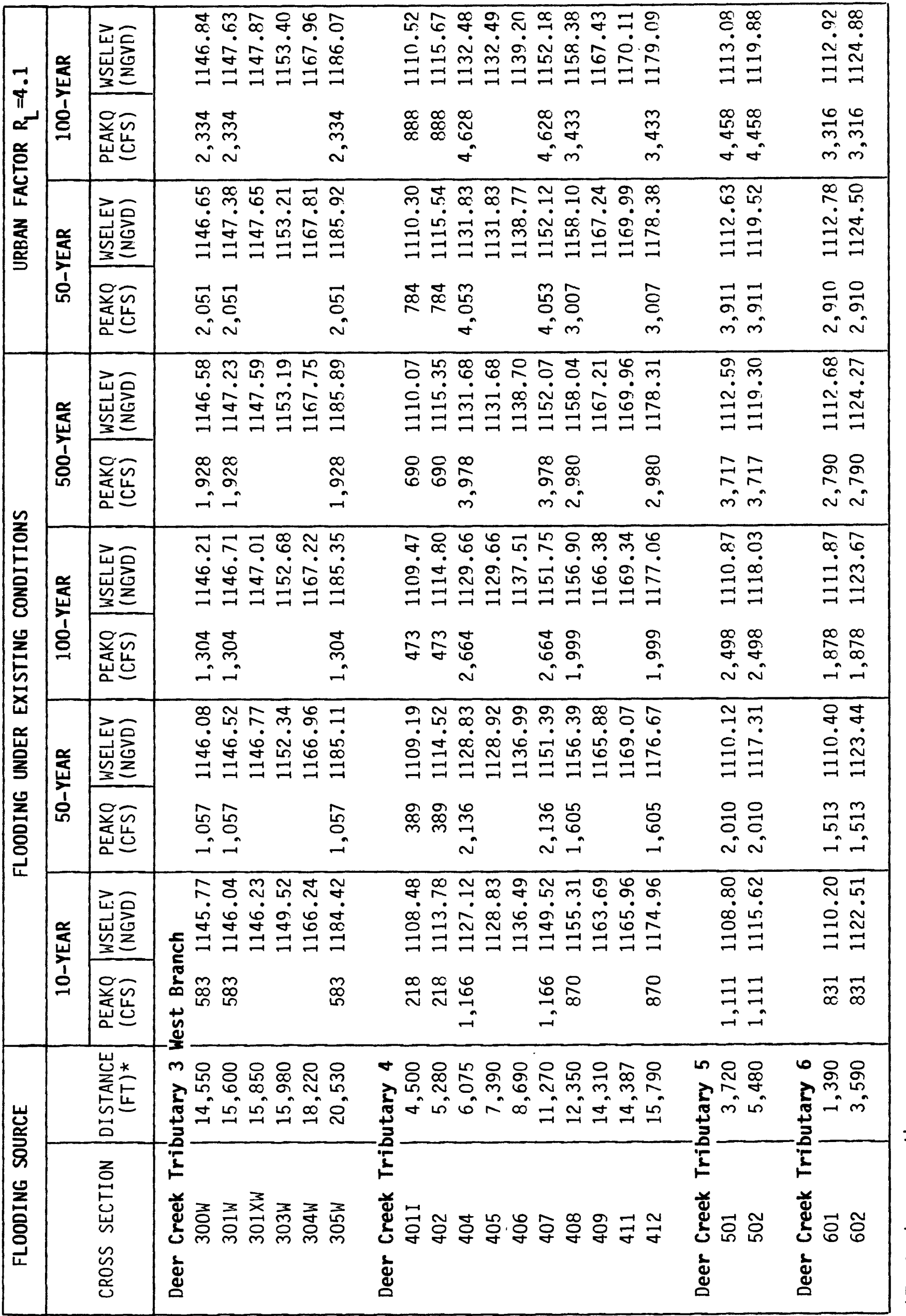




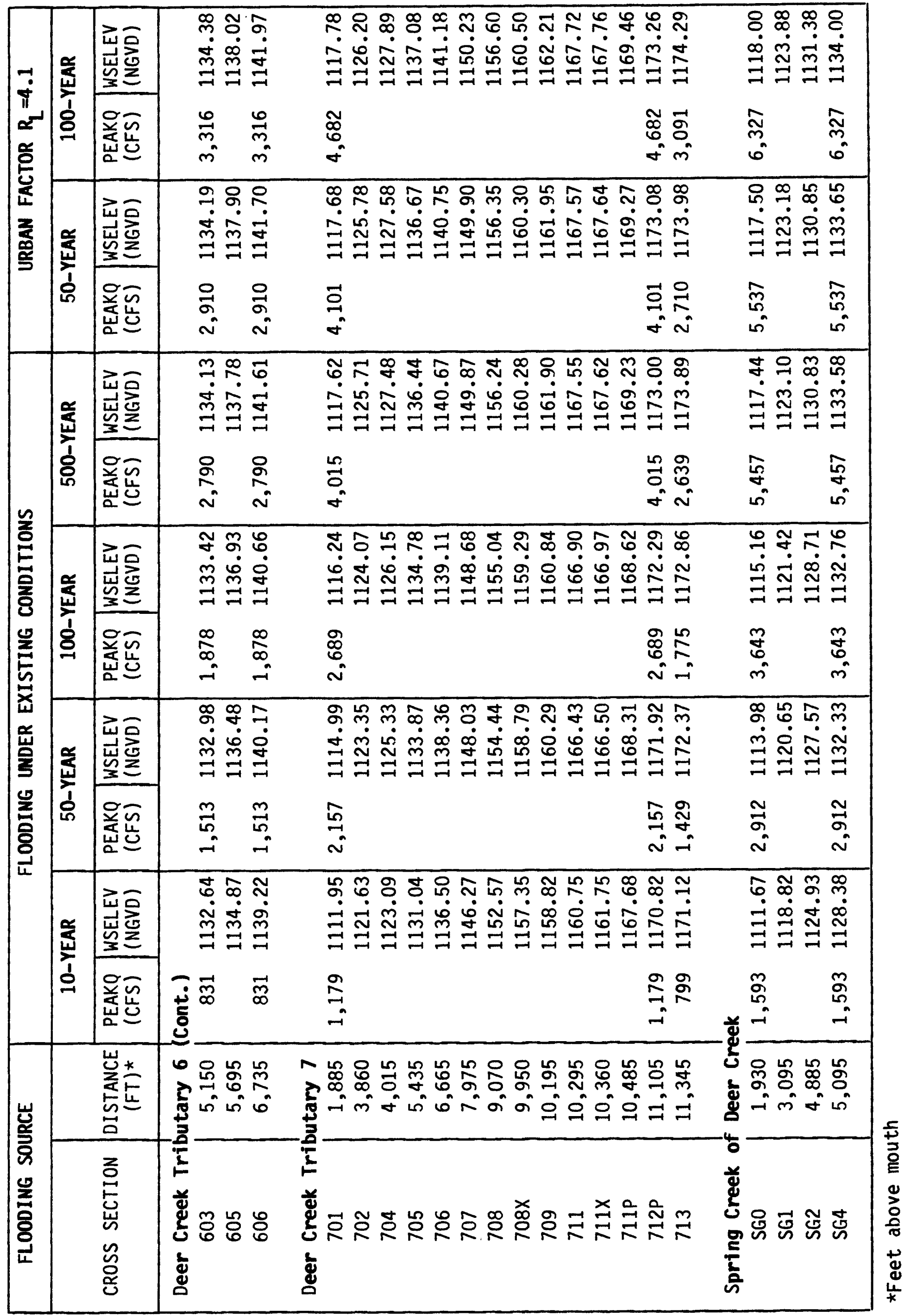




\begin{tabular}{|c|c|c|c|c|c|}
\hline \multirow{3}{*}{ 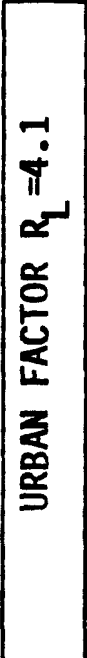 } & $\mid \begin{array}{c}\frac{0}{4} \\
\frac{1}{1} \\
\frac{1}{8} \\
0\end{array}$ & 岀员 & \multicolumn{2}{|l|}{ 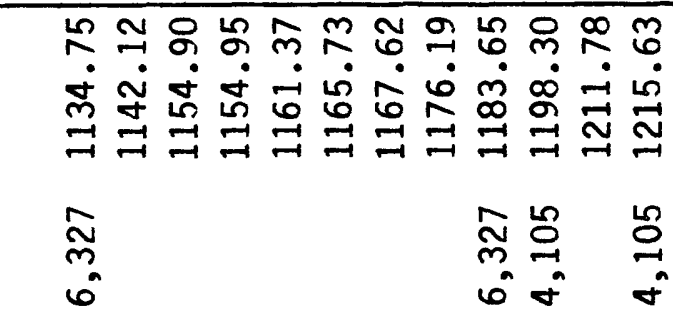 } & 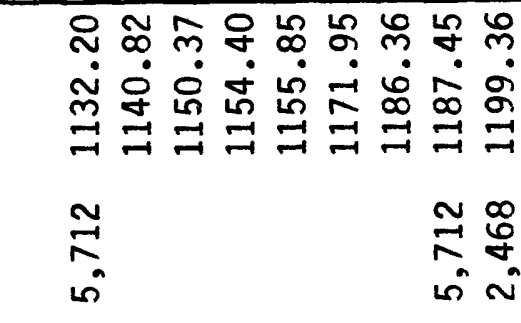 \\
\hline & \multirow{2}{*}{ 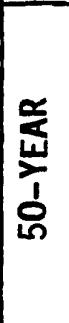 } & 崖邑 & \multirow{2}{*}{\multicolumn{2}{|c|}{ 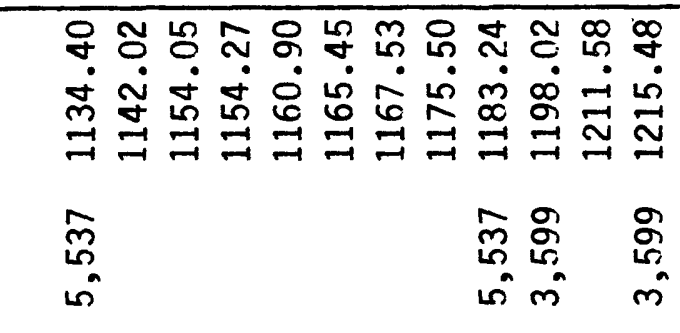 }} & \multirow{2}{*}{ 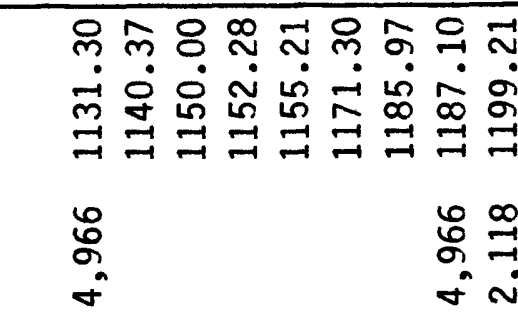 } \\
\hline & & 递 & & & \\
\hline \multirow{8}{*}{ 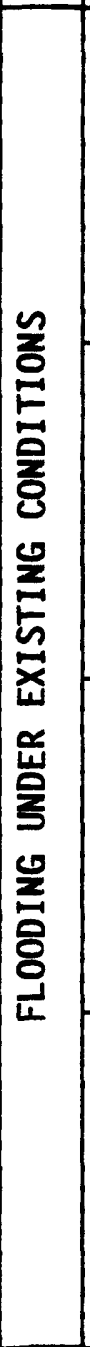 } & \multirow{2}{*}{$\begin{array}{l}\frac{0}{5} \\
\frac{1}{1} \\
\vdots \\
0 \\
0\end{array}$} & 岕苋 & \multirow{2}{*}{\multicolumn{2}{|c|}{ 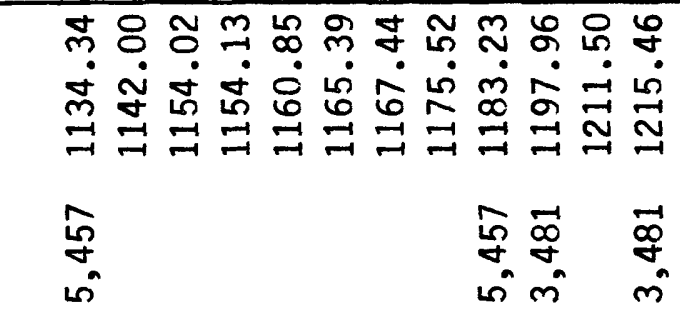 }} & \multirow{2}{*}{ 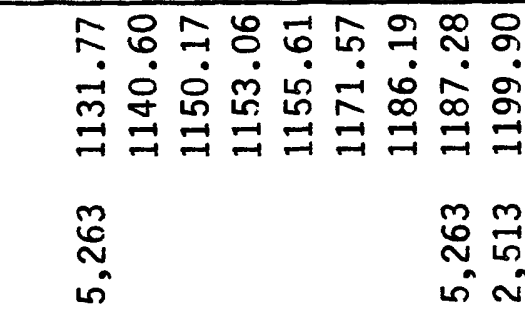 } \\
\hline & & 递岕 & & & \\
\hline & \multirow{2}{*}{$\begin{array}{l}\frac{1}{4} \\
\frac{1}{1} \\
0 \\
0 \\
0\end{array}$} & 岕 & \multirow{2}{*}{\multicolumn{2}{|c|}{ 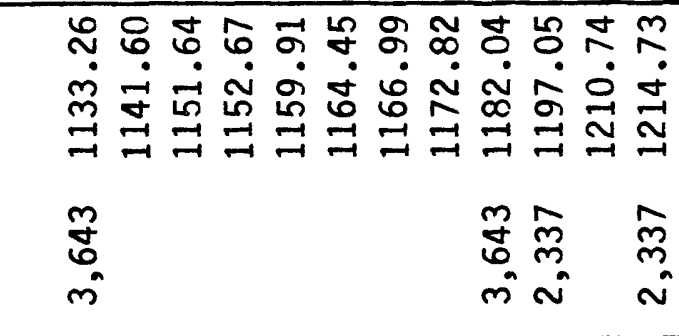 }} & \multirow{2}{*}{ 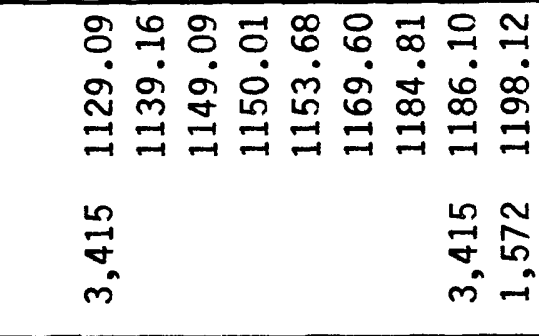 } \\
\hline & & 递苍 & & & \\
\hline & \multirow{2}{*}{ 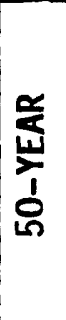 } & 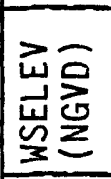 & \multirow{2}{*}{\multicolumn{2}{|c|}{ 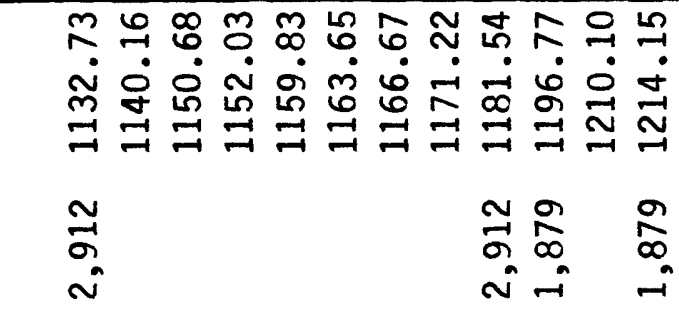 }} & \multirow{2}{*}{ 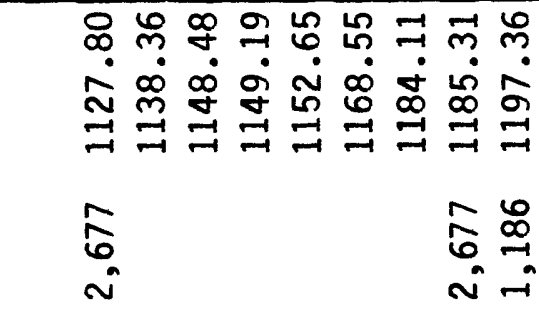 } \\
\hline & & 居芯 & & & \\
\hline & \multirow{2}{*}{$\underset{\frac{1}{2}}{\frac{1}{1}}$} & 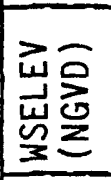 & \multirow{2}{*}{\multicolumn{2}{|c|}{ 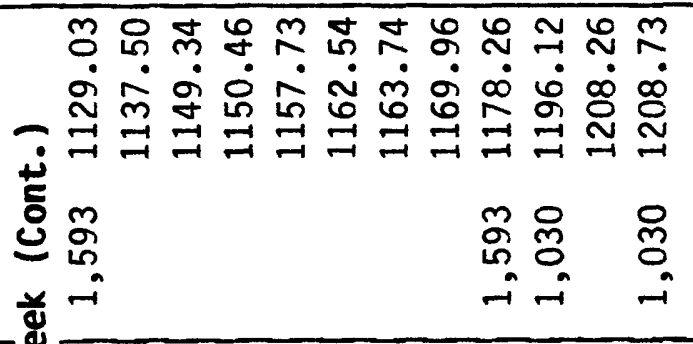 }} & \multirow{2}{*}{ 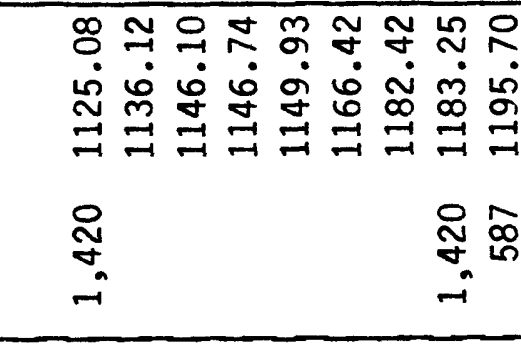 } \\
\hline & & 递芯 & & & \\
\hline \multirow{2}{*}{ 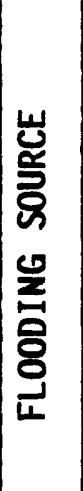 } & & 岕芯 & \multicolumn{2}{|l|}{ 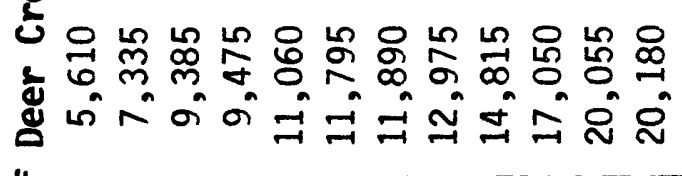 } & 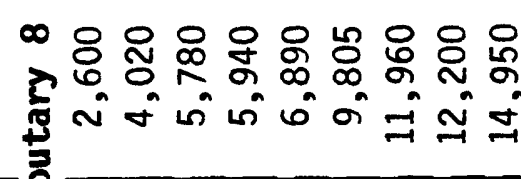 \\
\hline & & 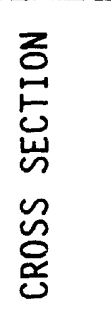 & 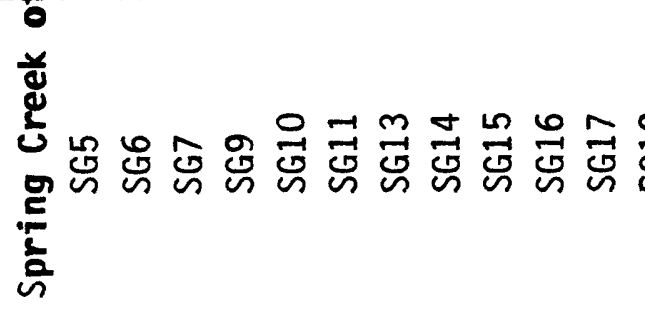 & & 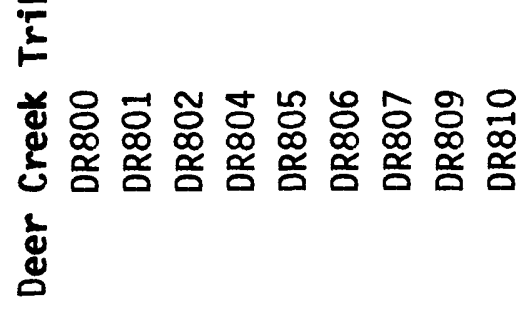 \\
\hline
\end{tabular}




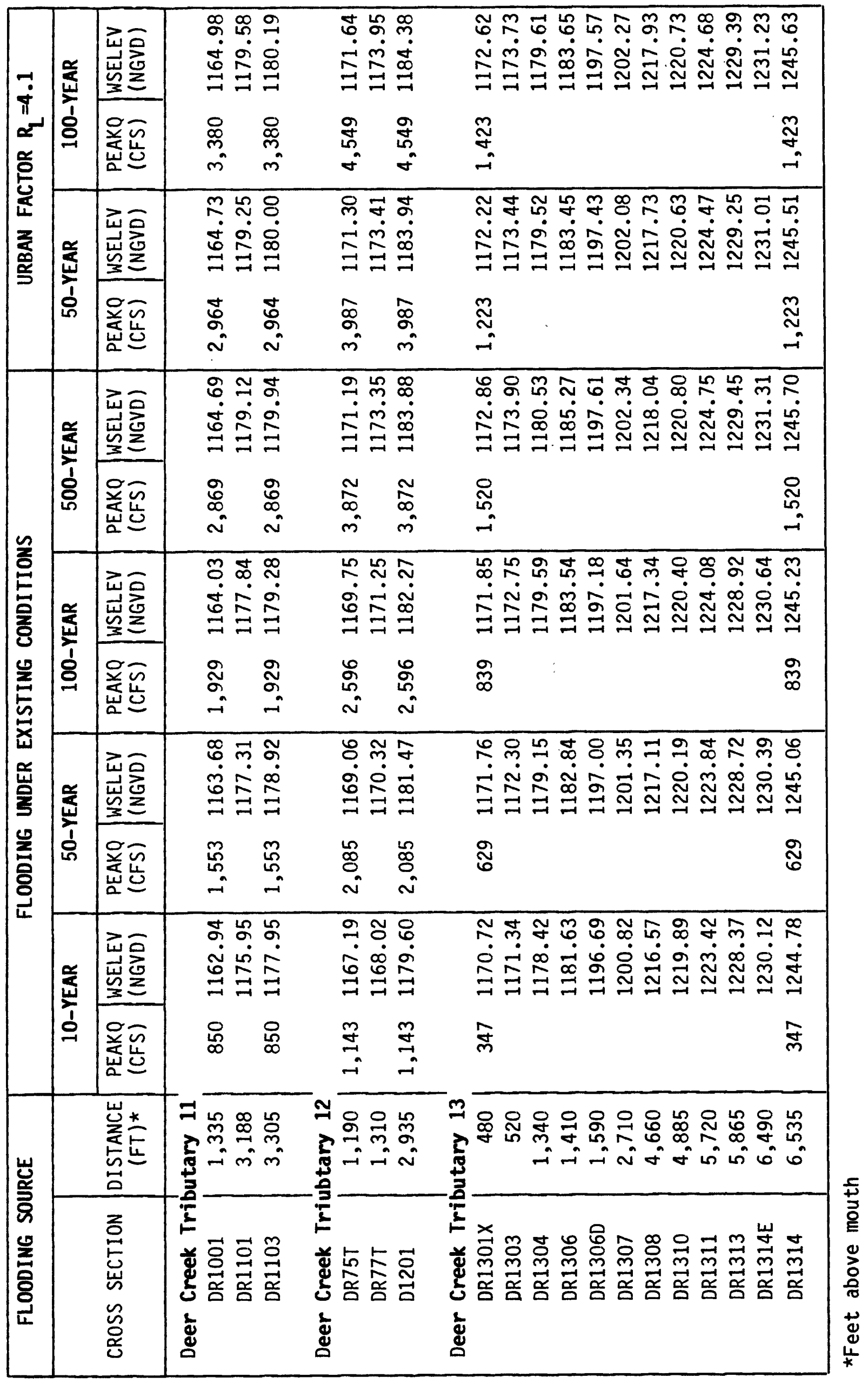




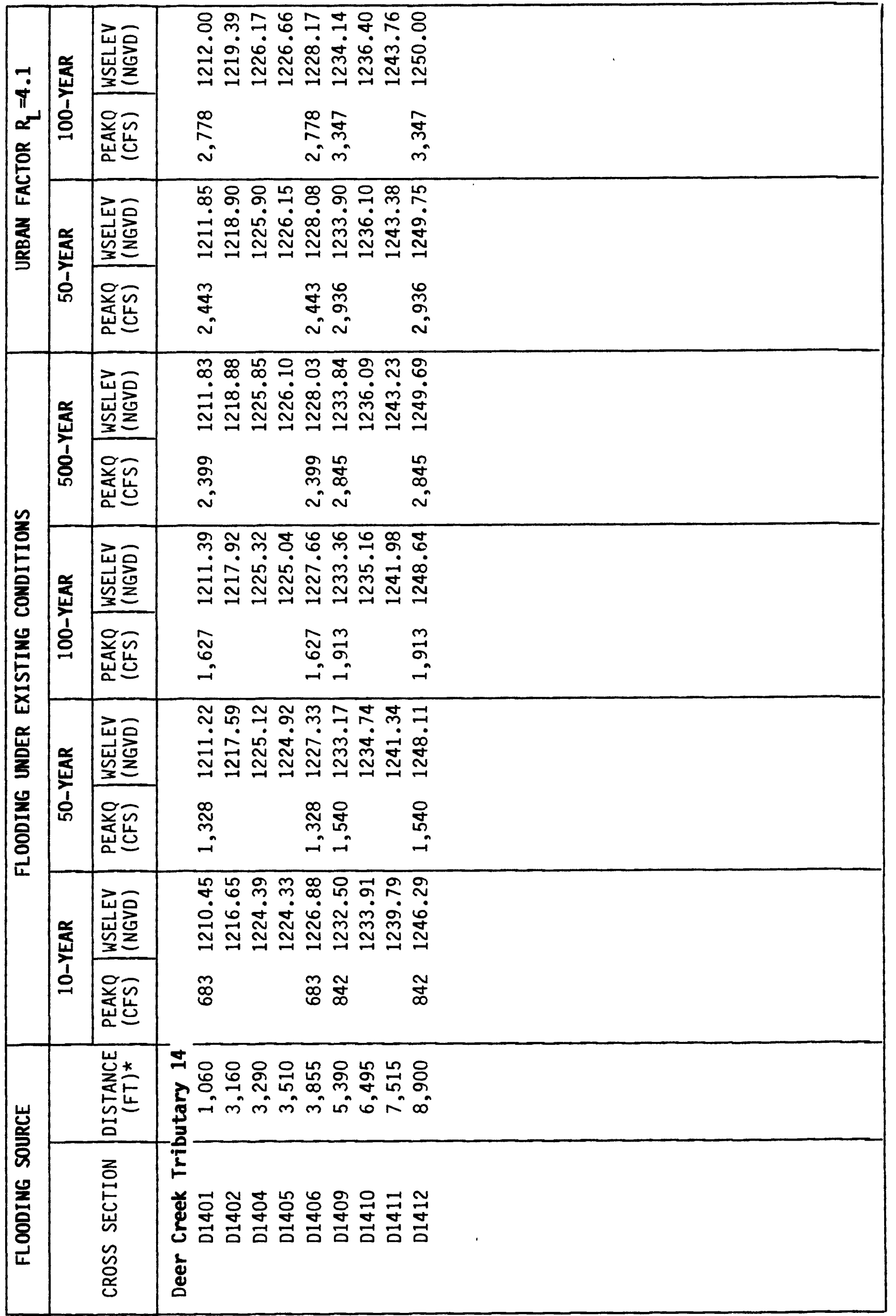




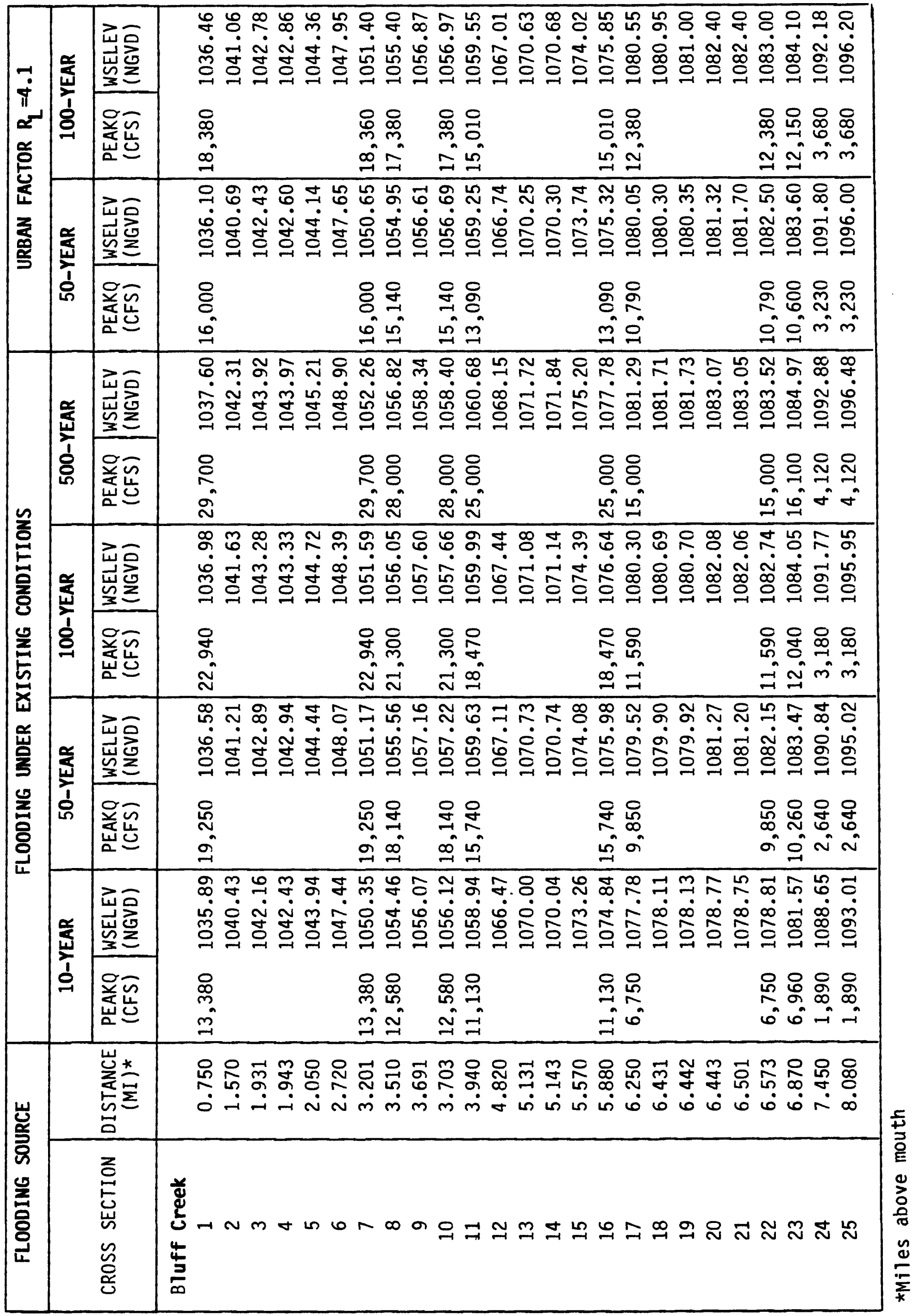




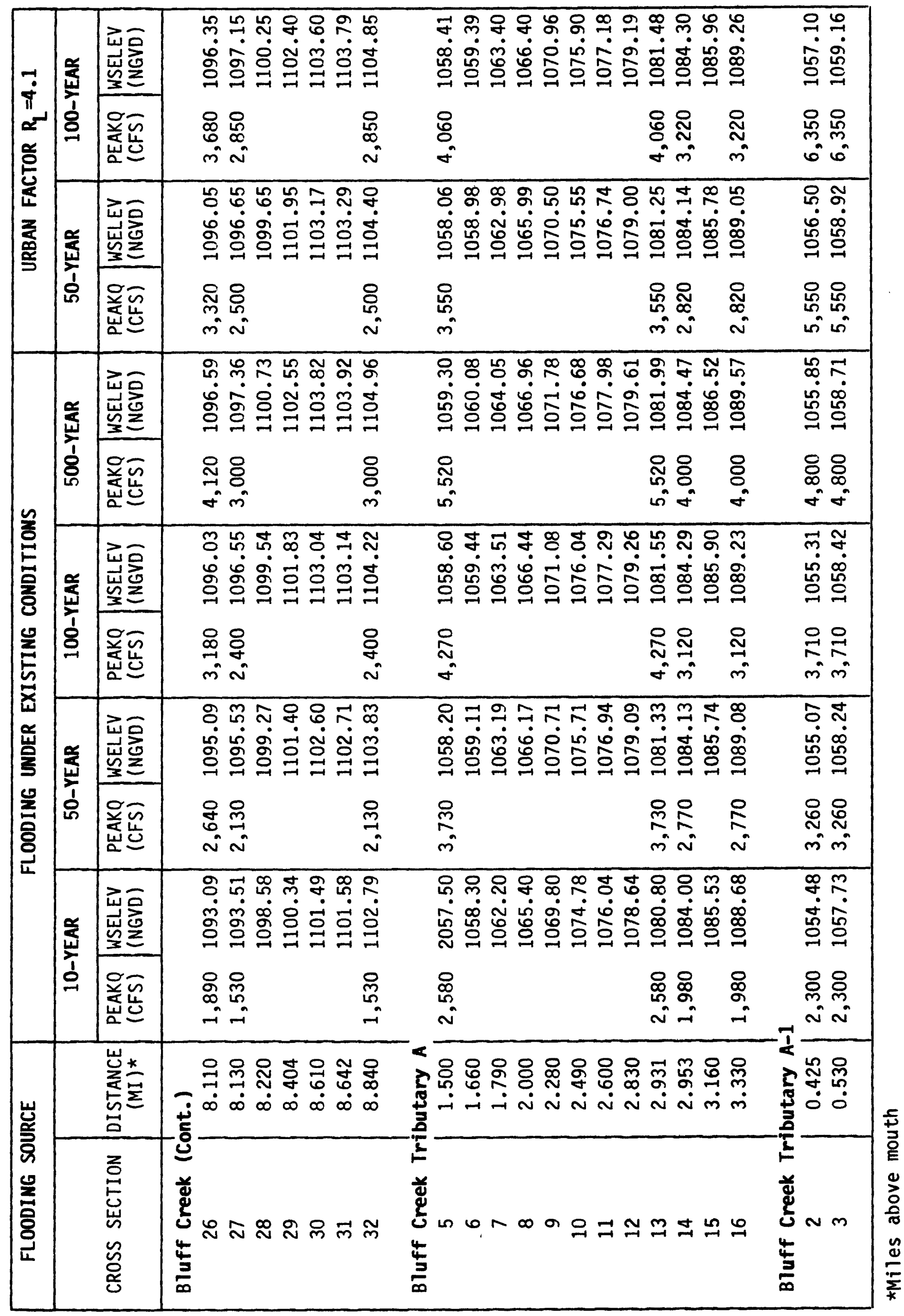




\begin{tabular}{|c|c|c|c|c|}
\hline \multirow{2}{*}{ 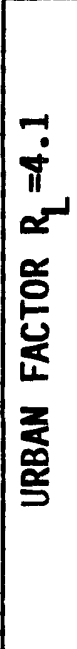 } & $\begin{array}{l}\frac{x}{4} \\
\frac{1}{1} \\
\delta \\
0\end{array}$ & 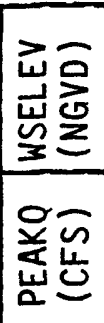 & \multicolumn{2}{|c|}{ 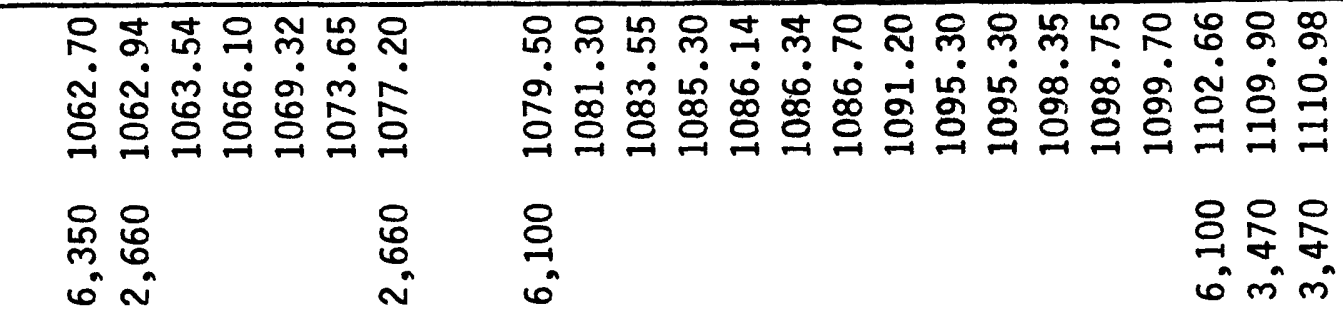 } \\
\hline & $\frac{a}{\frac{1}{5}}$ & 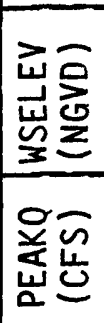 & 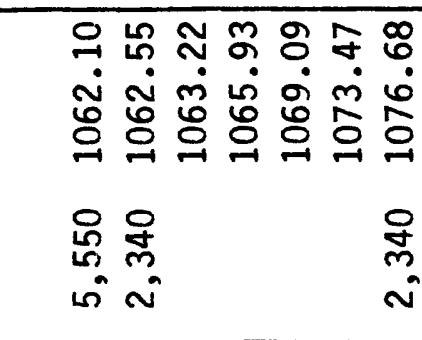 & 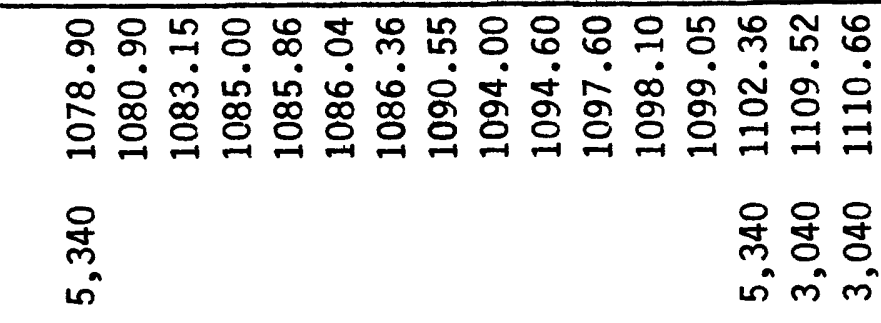 \\
\hline \multirow{4}{*}{ 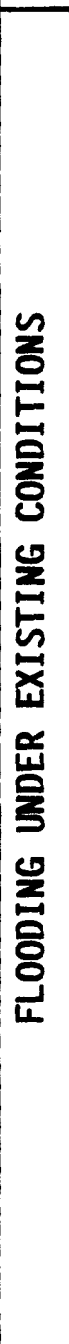 } & 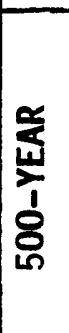 & 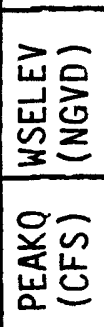 & 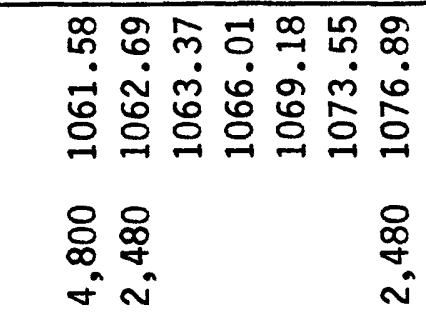 & 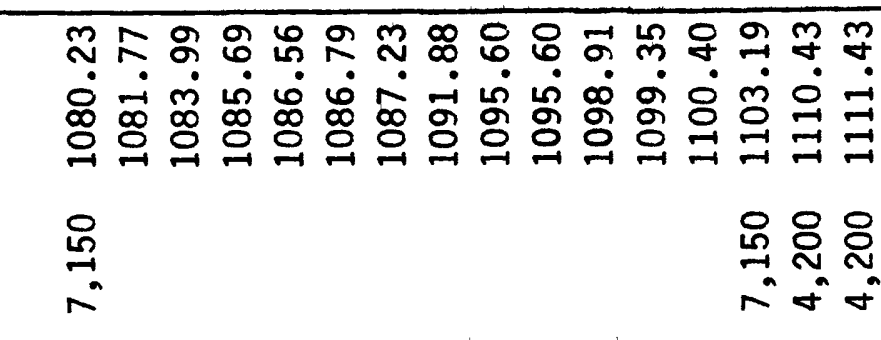 \\
\hline & $\begin{array}{l}\frac{\alpha}{5} \\
\frac{\mathbf{S}}{1} \\
0 \\
0\end{array}$ & 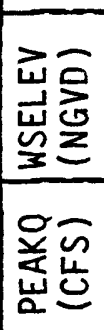 & 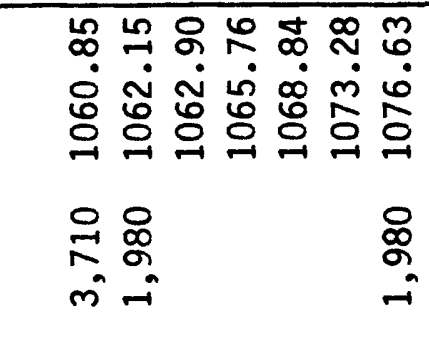 & 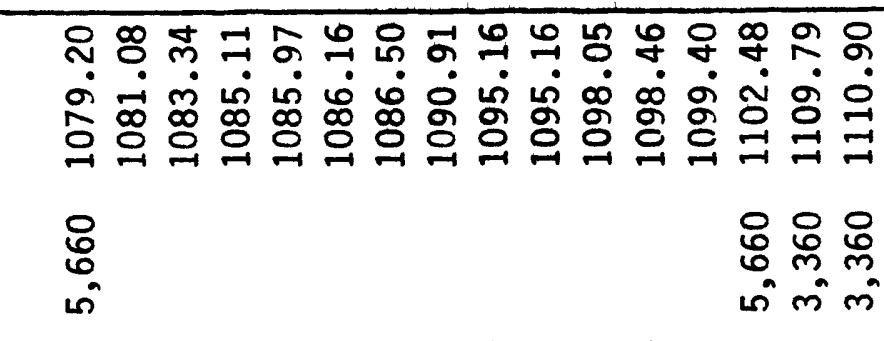 \\
\hline & $\frac{\mathfrak{y}}{5}$ & 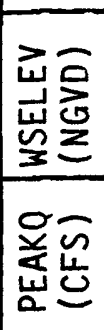 & 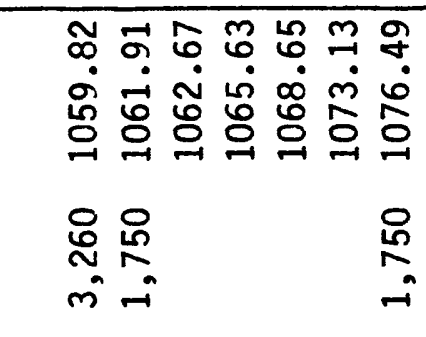 & 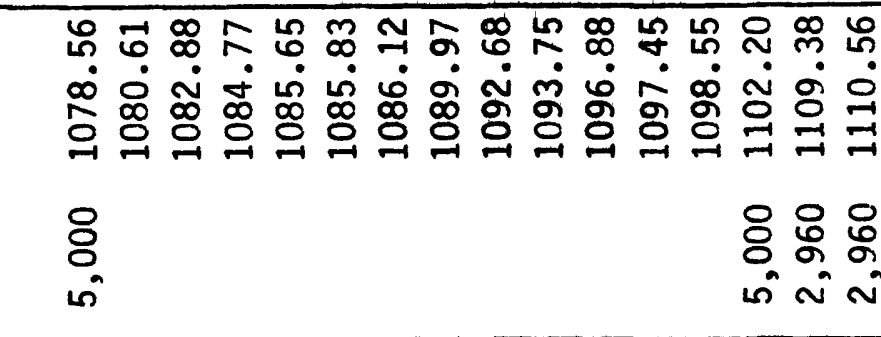 \\
\hline & $\frac{a}{\frac{a}{2}}$ & 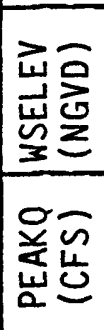 & 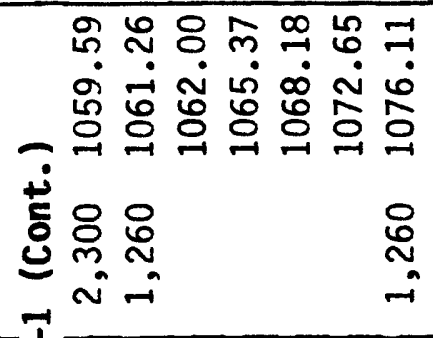 & 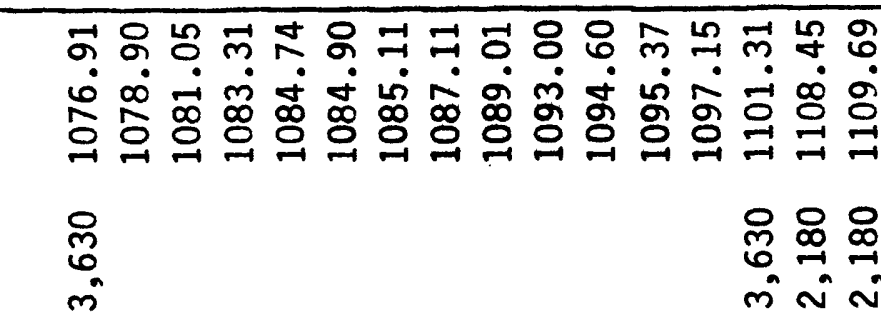 \\
\hline \multirow{2}{*}{ 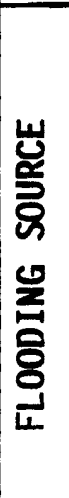 } & & 岕岕点 & 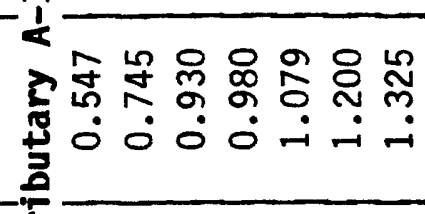 & 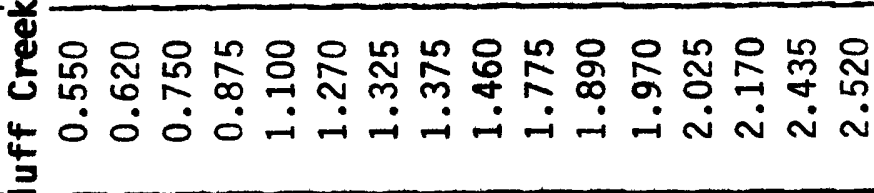 \\
\hline & & Z & 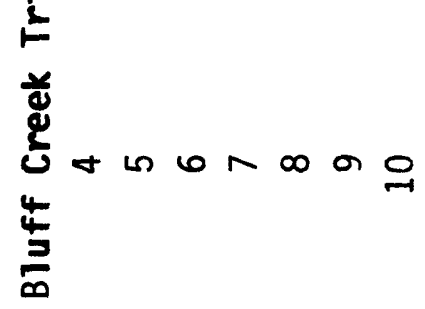 & 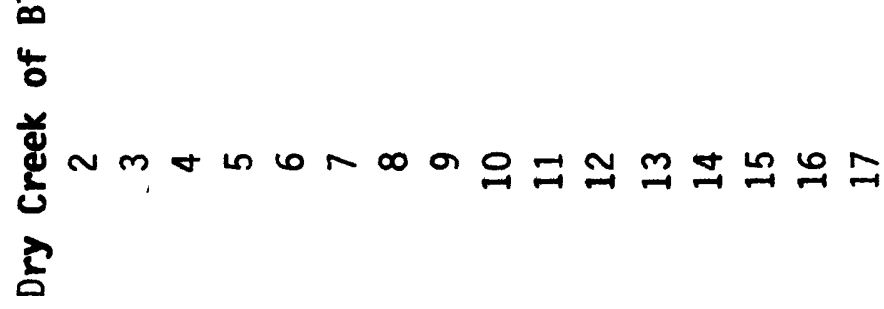 \\
\hline
\end{tabular}




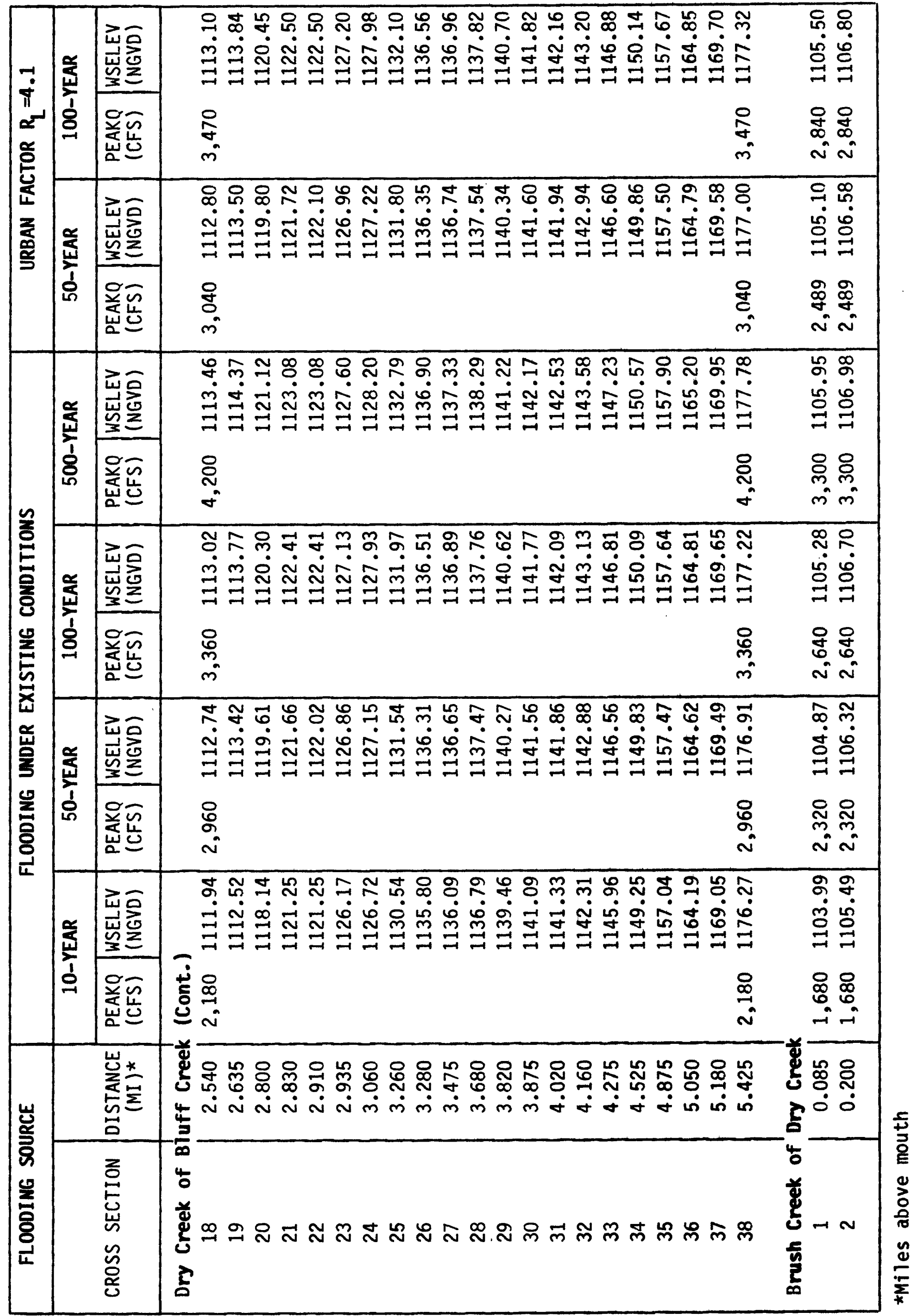




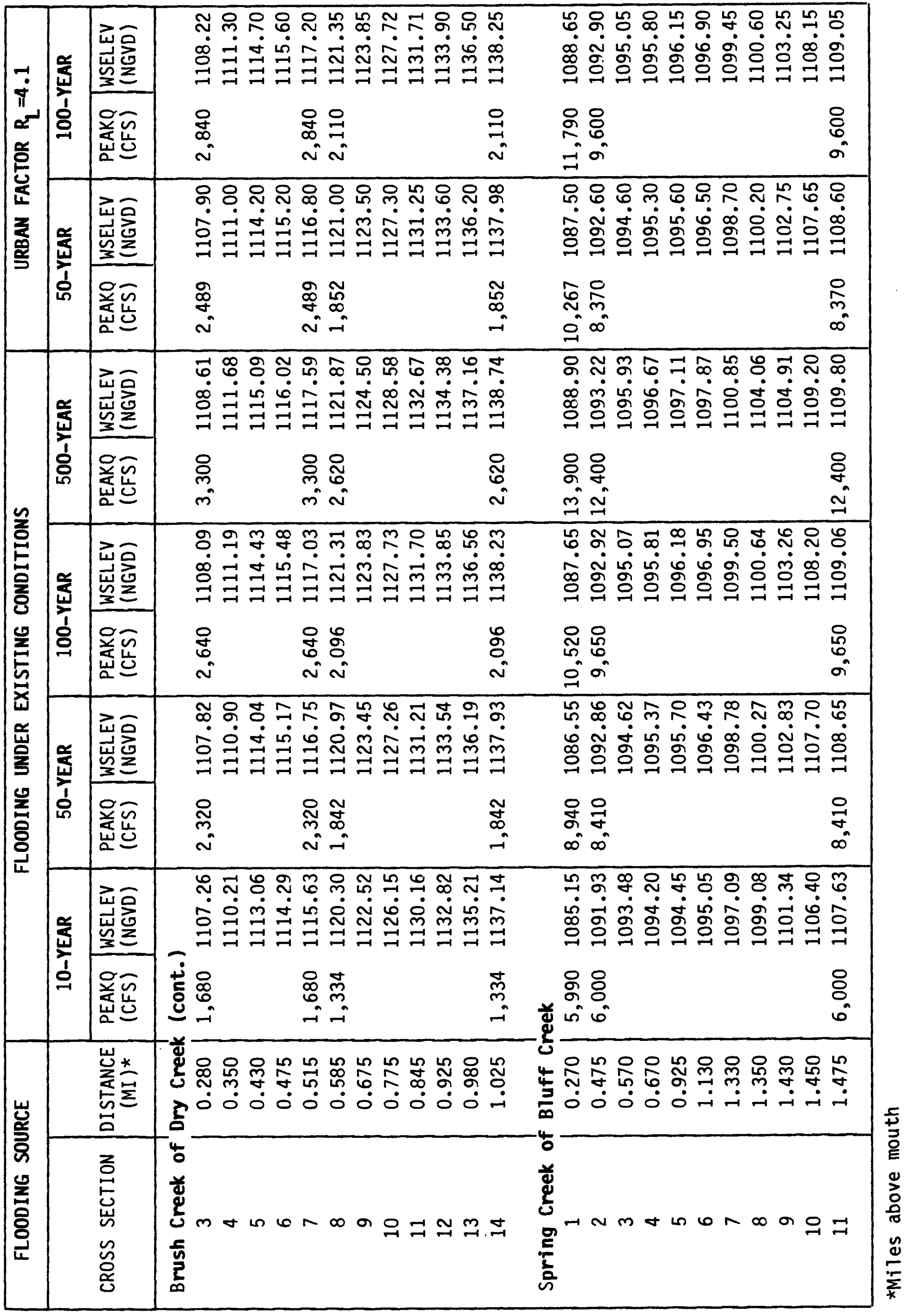




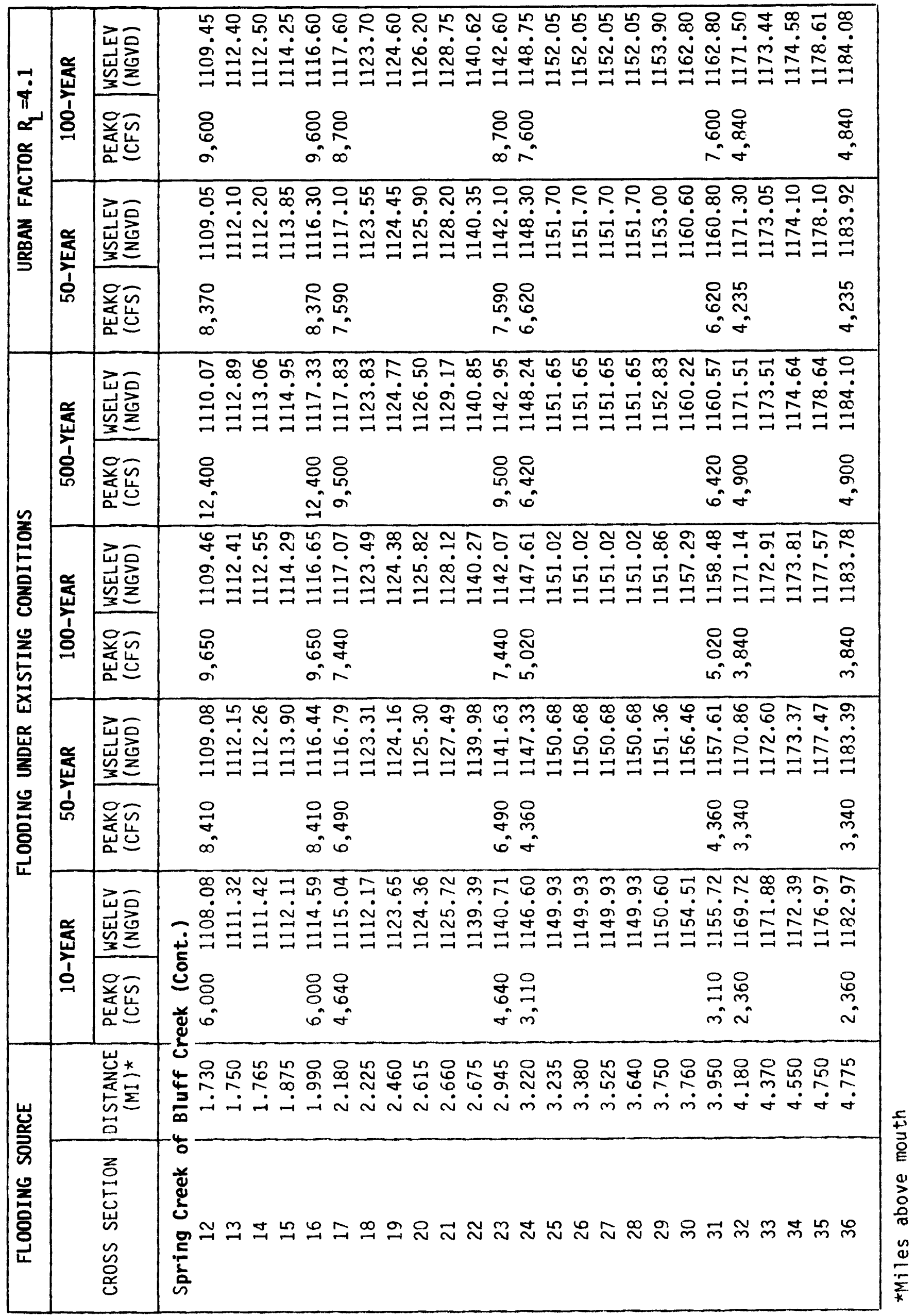




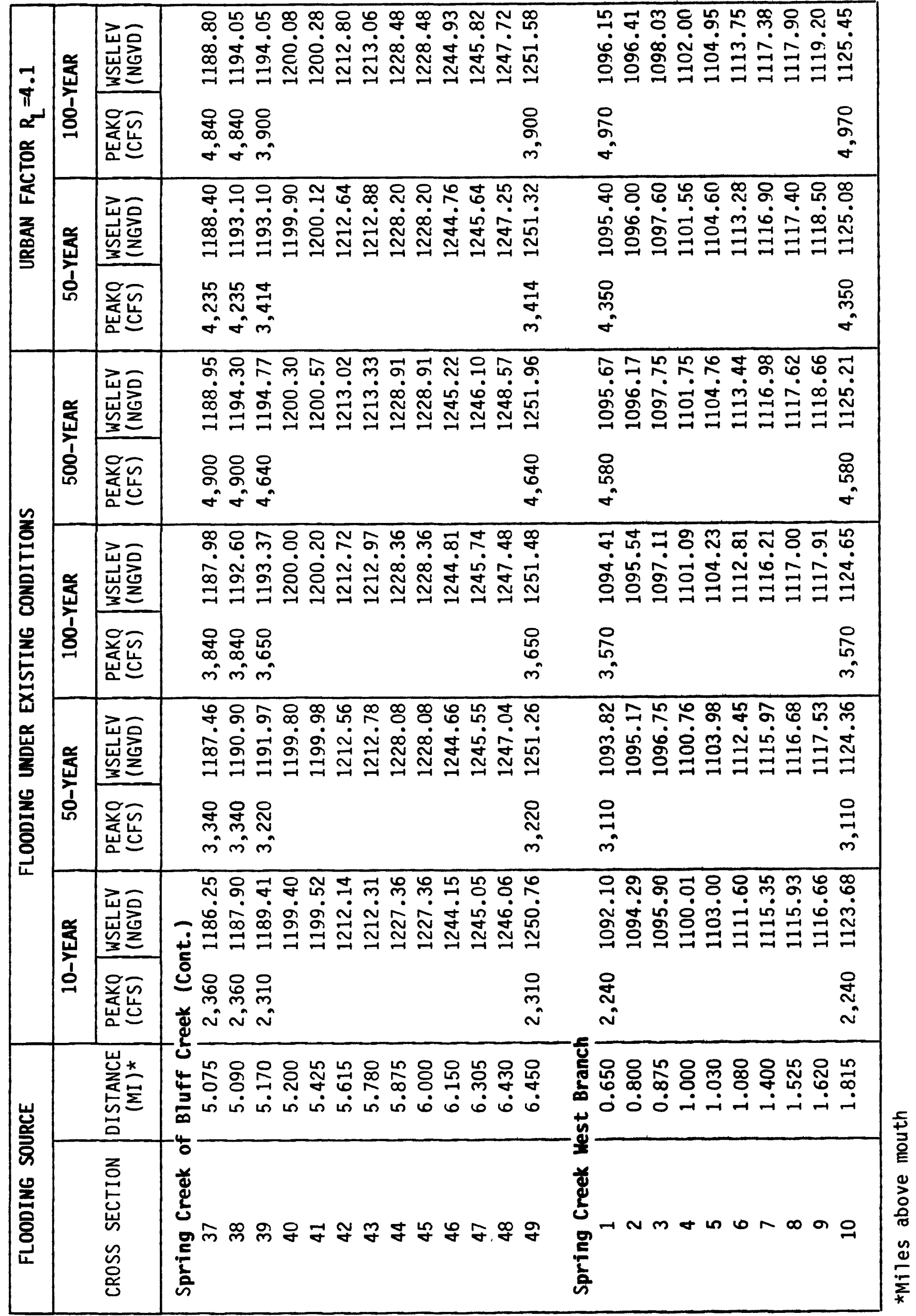




\begin{tabular}{|c|c|c|c|}
\hline \multirow{4}{*}{ 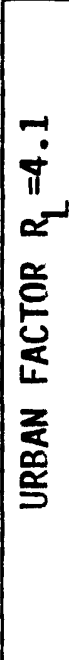 } & \multirow{2}{*}{ 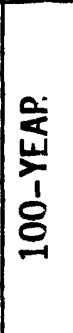 } & 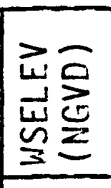 & 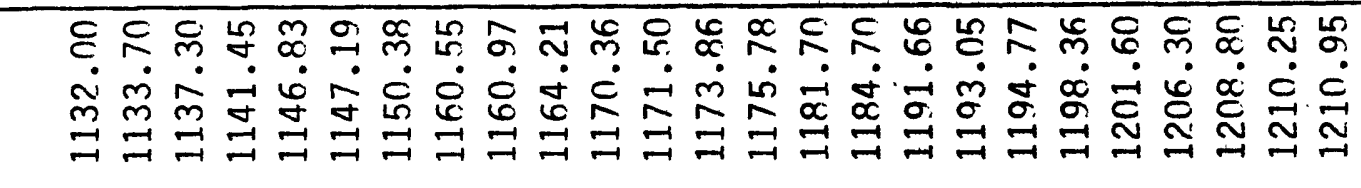 \\
\hline & & 養岕 & 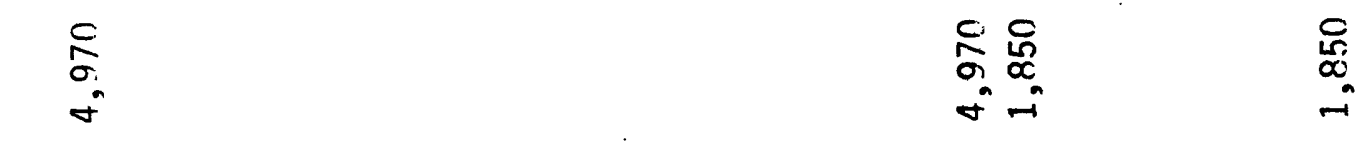 \\
\hline & \multirow{2}{*}{ 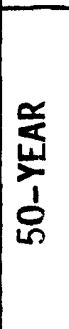 } & 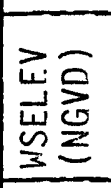 & 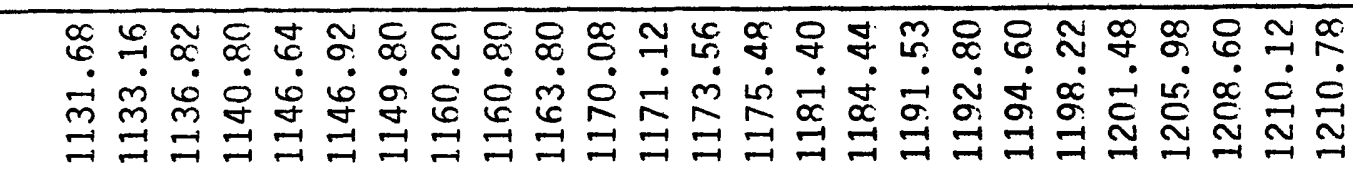 \\
\hline & & 递岕 & 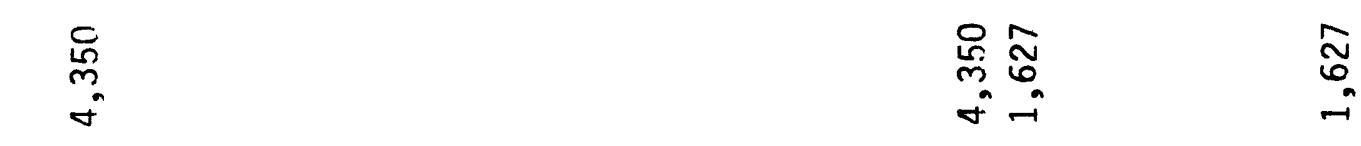 \\
\hline \multirow{8}{*}{ 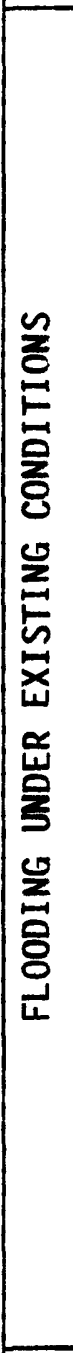 } & \multirow{2}{*}{$\frac{\substack{\frac{1}{4} \\
\frac{1}{1}}}{8}$} & 崖全 & \multirow{2}{*}{ 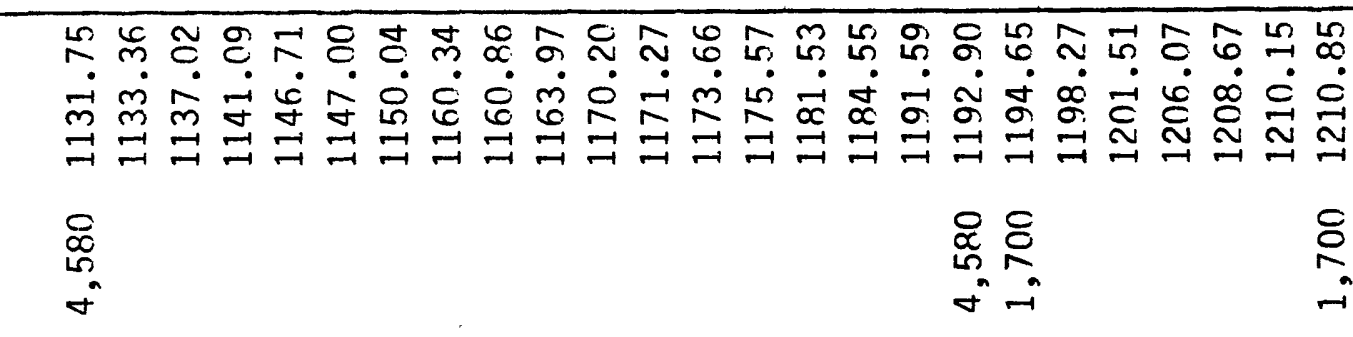 } \\
\hline & & 总䓽 & \\
\hline & \multirow{2}{*}{ 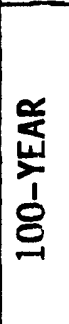 } & 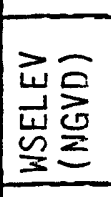 & \multirow{2}{*}{ 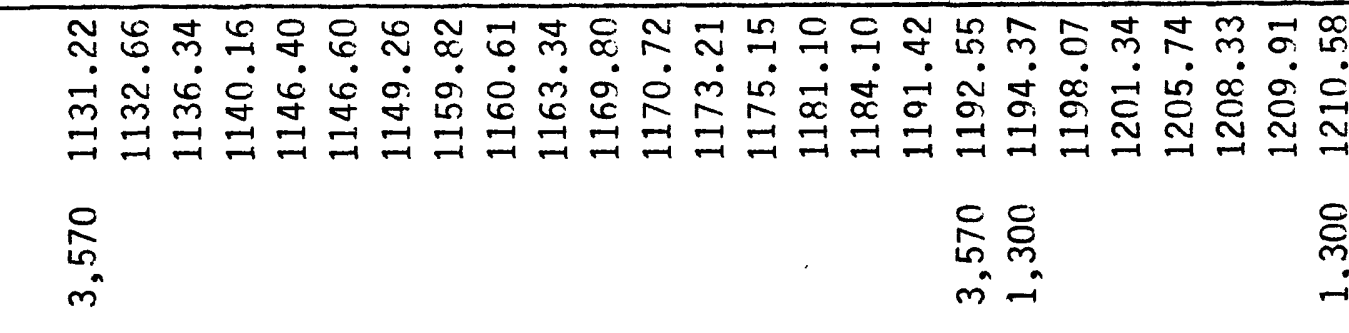 } \\
\hline & & 迹慈 & \\
\hline & \multirow{2}{*}{ 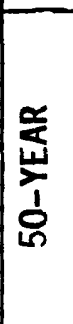 } & 崖 & 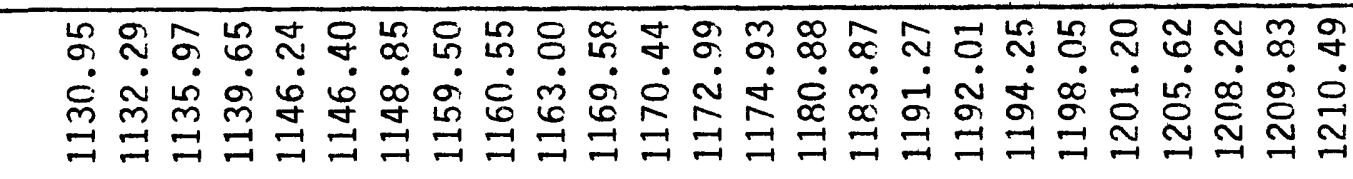 \\
\hline & & 运 & 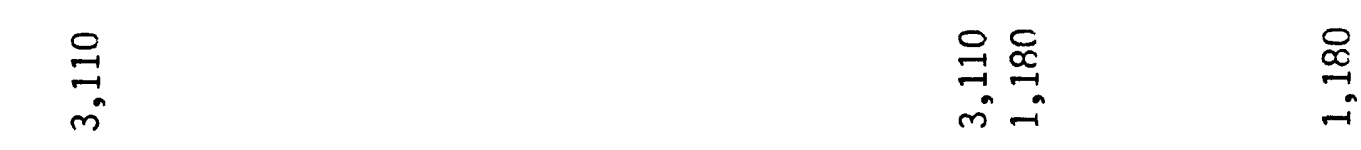 \\
\hline & \multirow{2}{*}{ 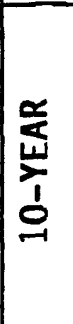 } & 岂量 & 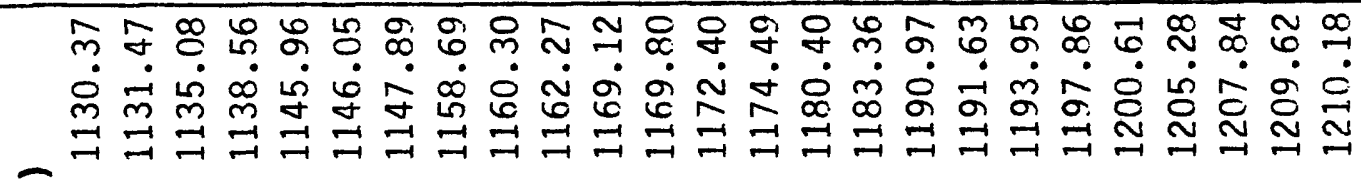 \\
\hline & & 善出 & 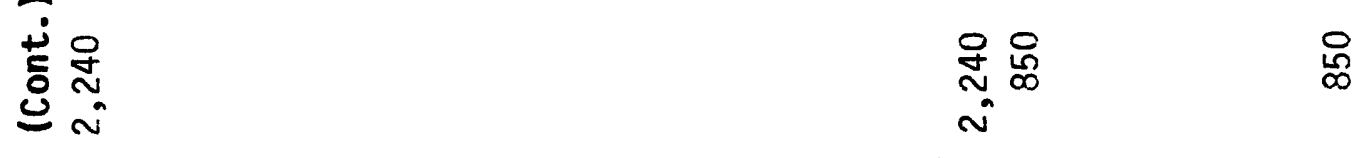 \\
\hline \multirow{2}{*}{ 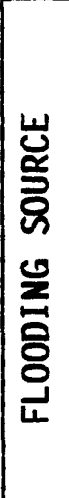 } & & 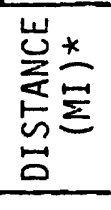 & $\begin{array}{l} \\
\end{array}$ \\
\hline & & 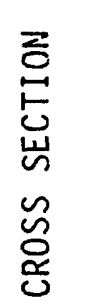 & 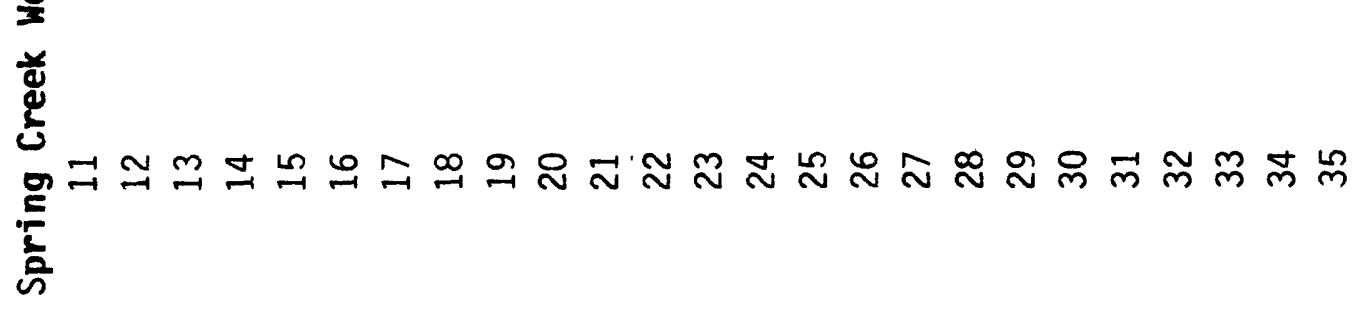 \\
\hline
\end{tabular}




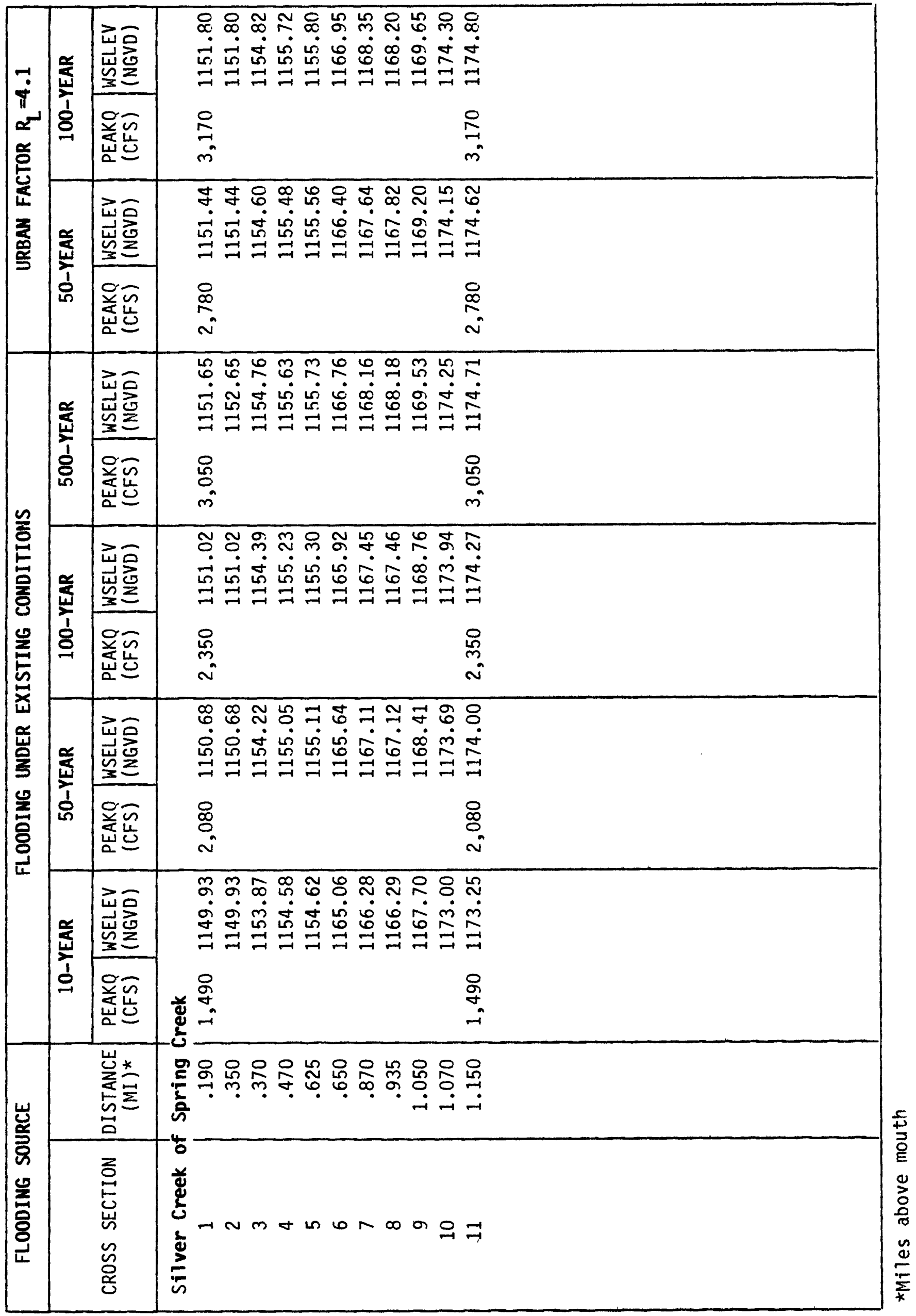




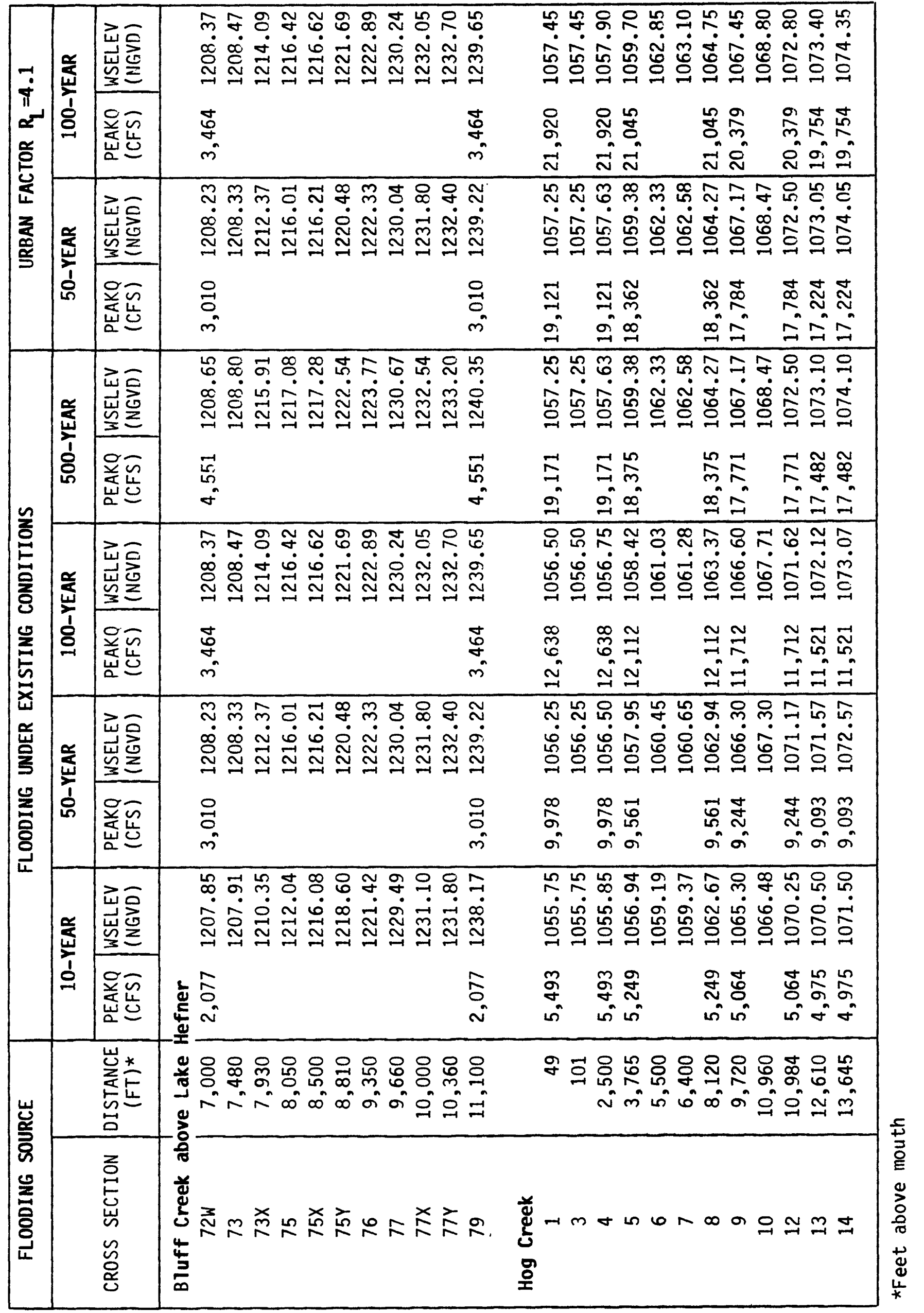




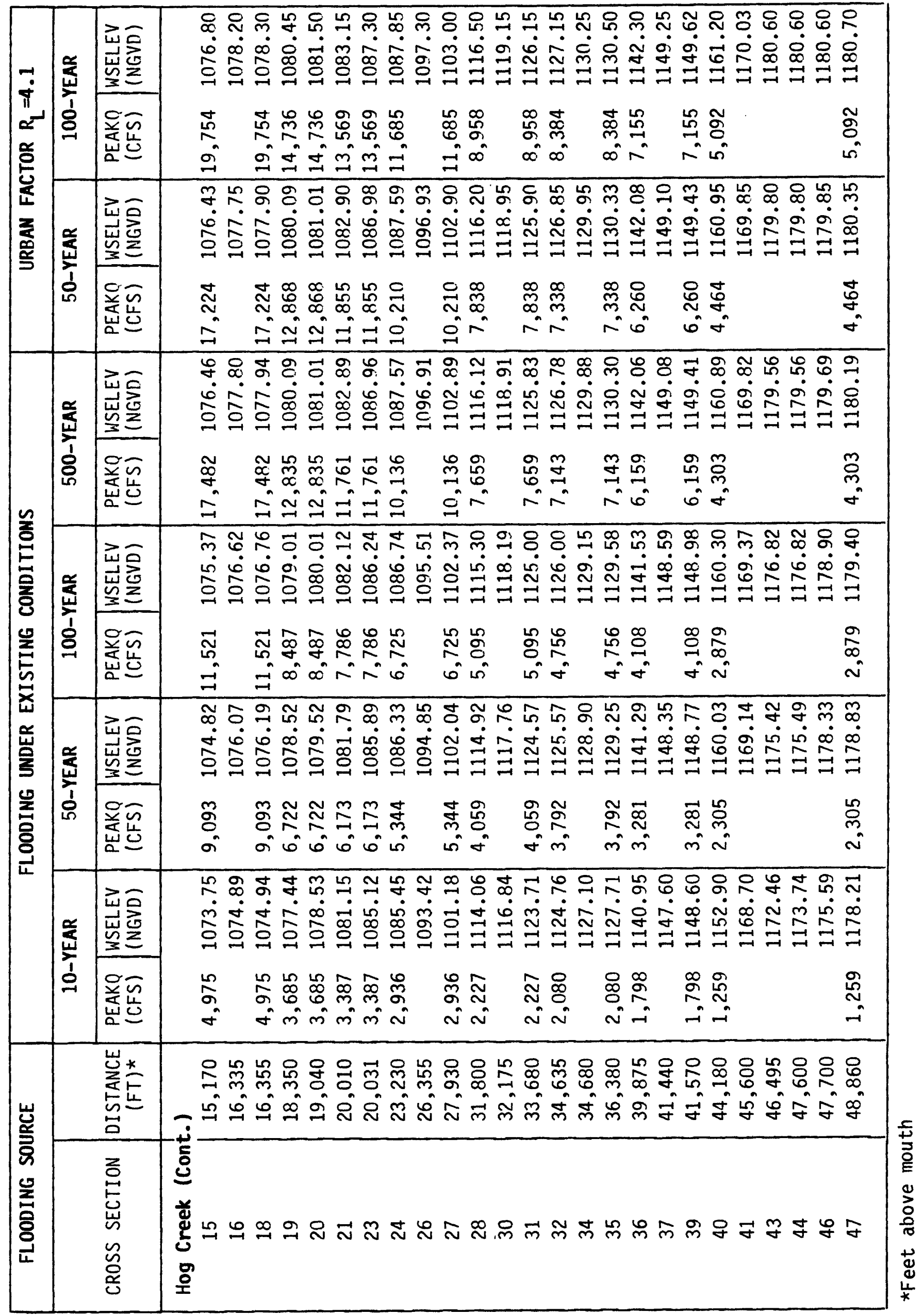




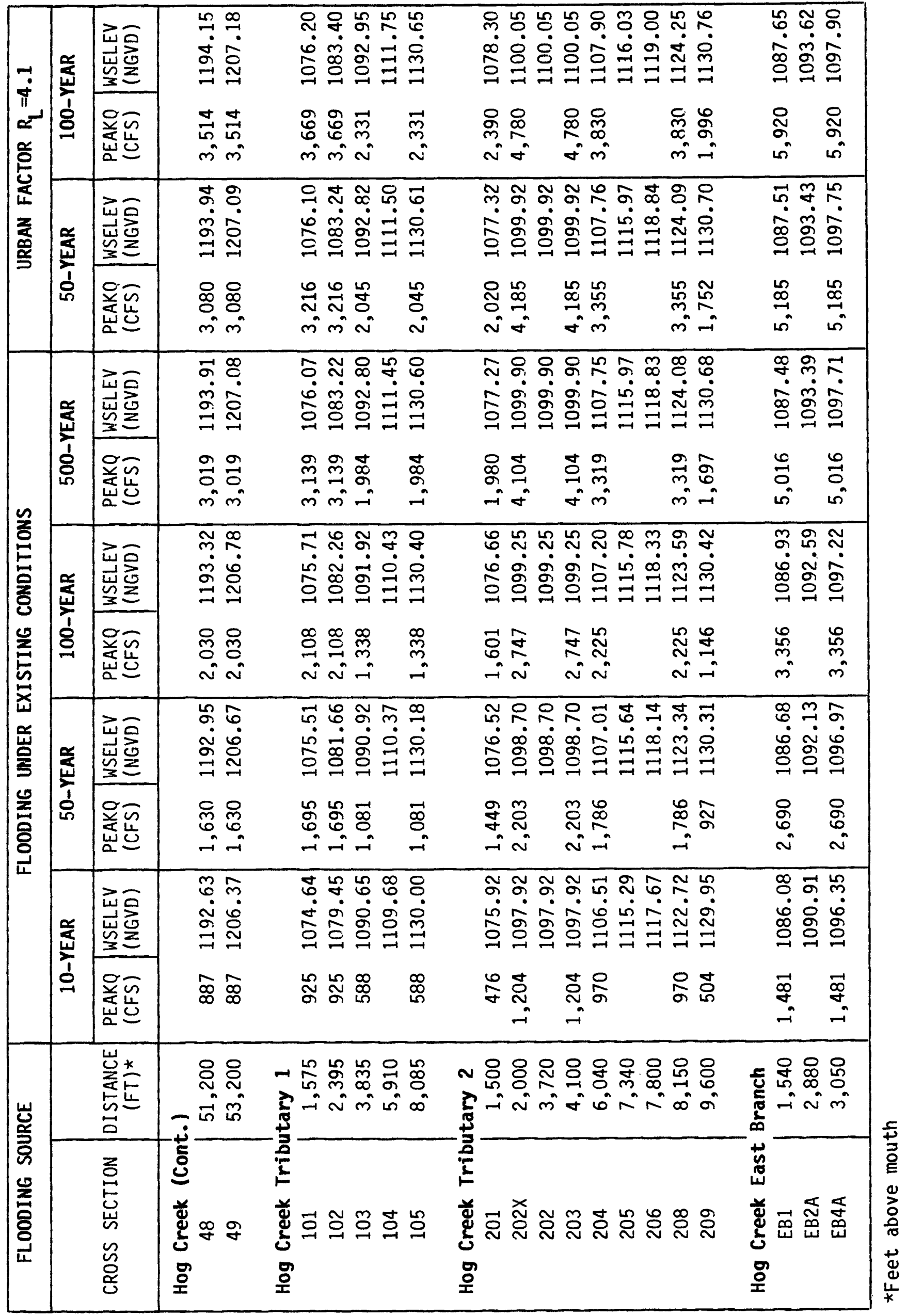




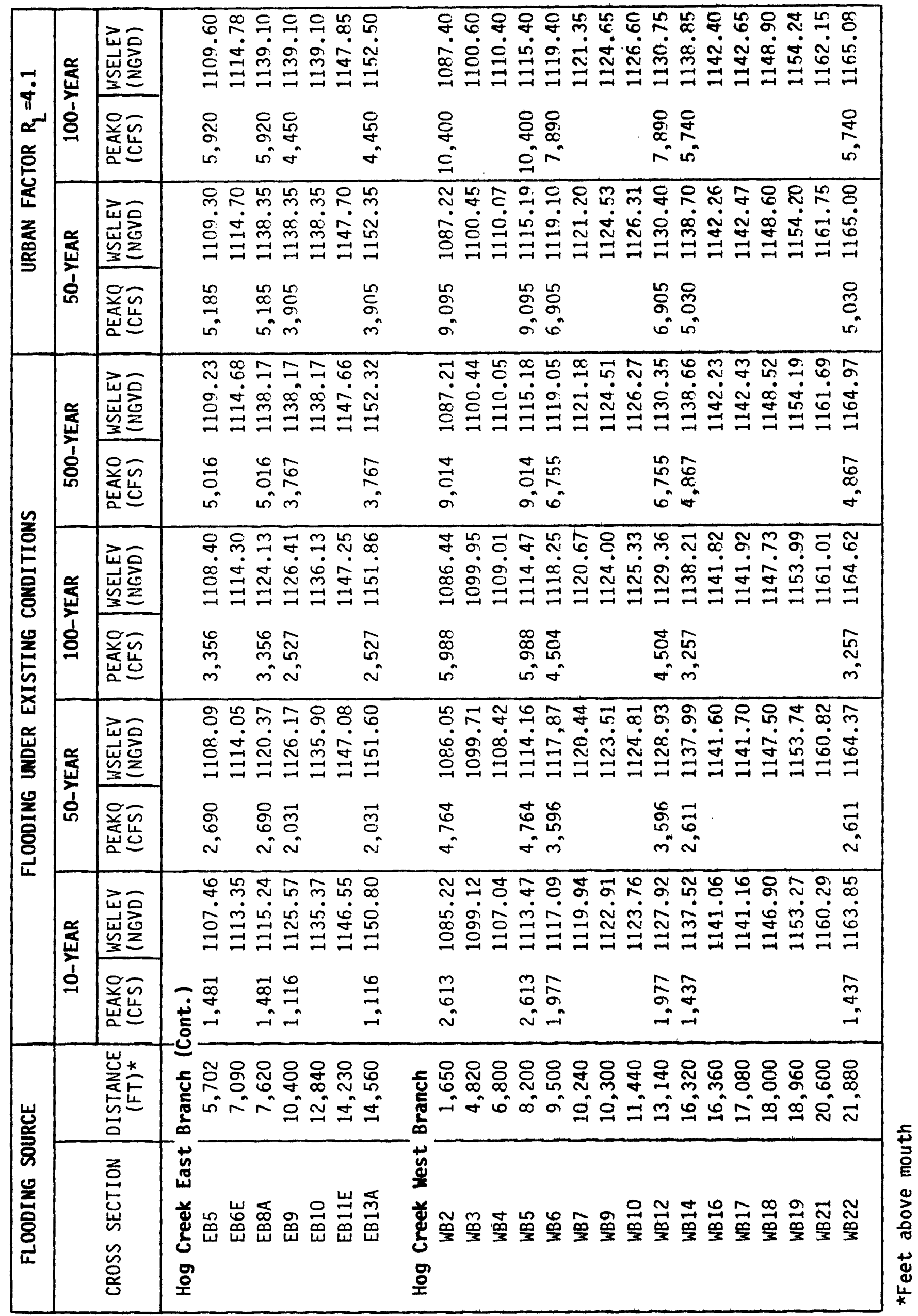




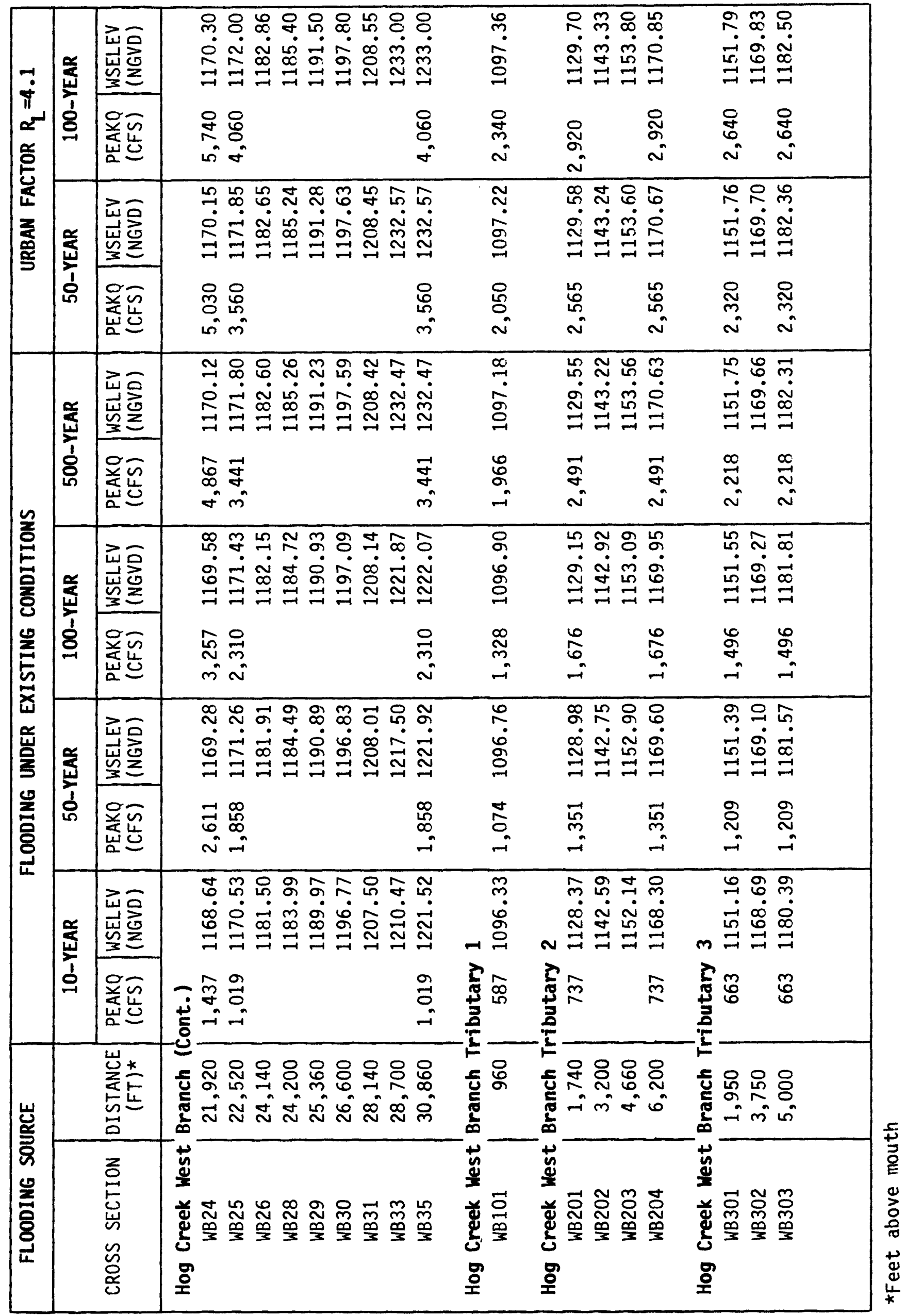




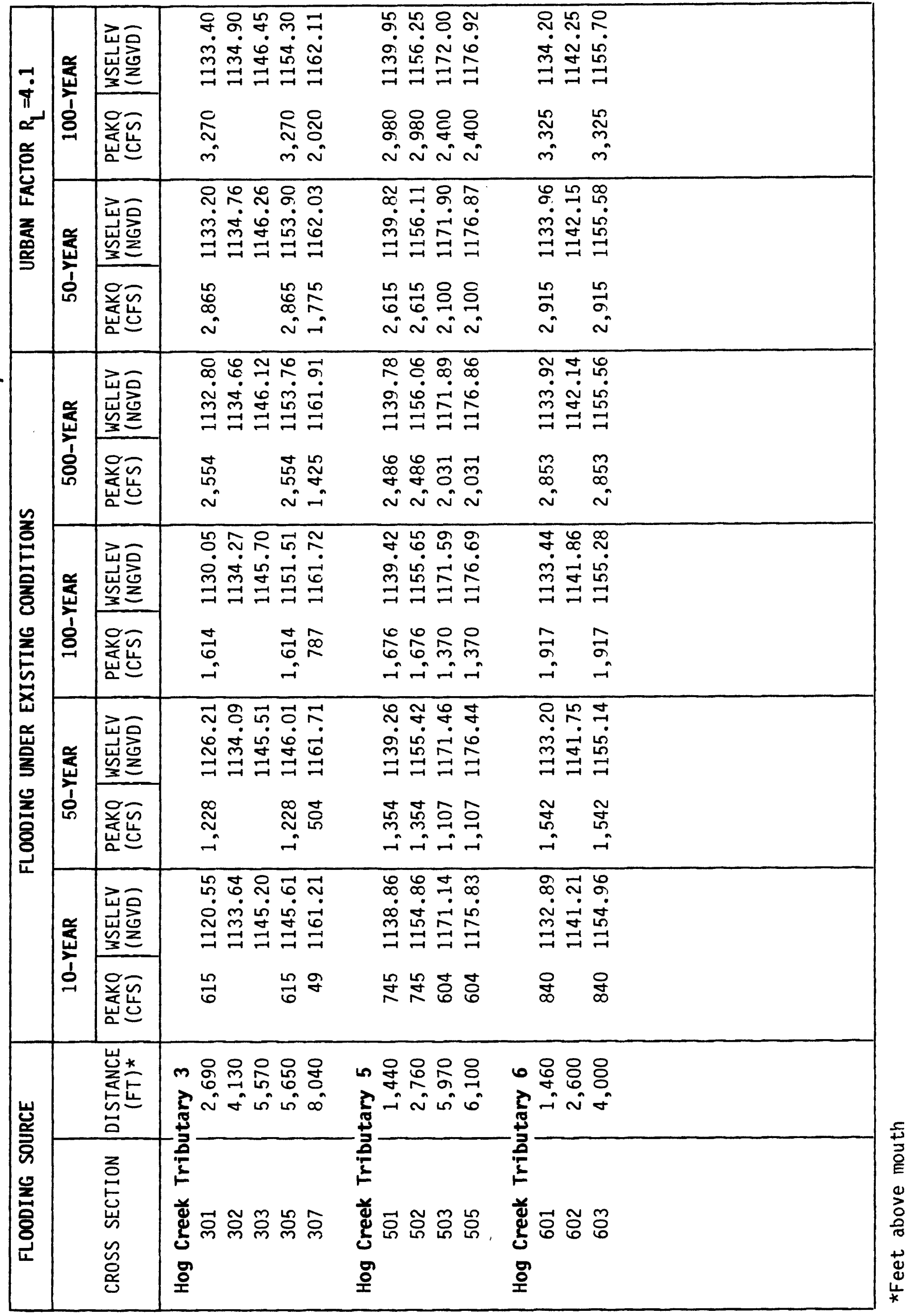




\section{REFERENCES CITED}

Federa1 Emergency Management Agency, 1982, City of Ok1ahoma City, Ok1ahoma:

Flood insurance study, community number 405378.

Huntzinger, T. L., 1978, Application of hydraulic and hydrologic data in urban stormwater management: U.S. Geological Survey Open-File Report 78-414, $33 \mathrm{p}$.

Leopold, L. G., 1968, Hydrology for urban and land planning--A guidebook on the hydrologic effects of urban land use: U.S. Geological Survey Circu$\operatorname{lar} 554,18 \mathrm{p}$.

Sauer, V. B., 1974, An approach to estimating flood frequency for urban areas in 0klahoma: U.S. Geological Survey Water-Resources Investigations 23-74, $10 \mathrm{p}$.

Thomas, W. 0., Jr., and Corley, R. K., 1977, Techniques for estimating flood discharges for Oklahoma streams: U.S. Geological Survey Water-Resources Investigations $77-54,170 \mathrm{p}$. 
Bluff Creek Tributary A...................... 14, 75

Bluff Creek Tributary A-1................... 14, 75, 76

Bluff Creek above Lake Hefner.................... 14, 83

Brock Creek.................................... 9, 26, 27

Brush Creek of Dry Creek.......................... 14,77,78

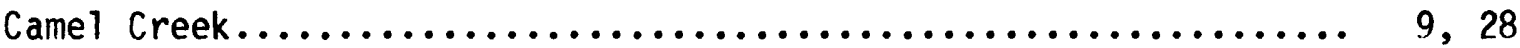

Camel Creek East Branch.......................... 9, 29

Camel Creek Middle Branch........................ 9, 29

Canadian River Tributary $1 \ldots \ldots \ldots \ldots \ldots \ldots \ldots \ldots \ldots \ldots \ldots \ldots \ldots \ldots \ldots \ldots$

Tributary 0 of Canadian River Tributary $1 \ldots \ldots \ldots \ldots \ldots$....... 39

Tributary 1 of Canadian River Trihutary $1 \ldots \ldots \ldots \ldots \ldots \ldots$, 38

Tributary 2 of Canadain River Tributary $1 \ldots \ldots \ldots \ldots \ldots$......... 38

Tributary 3 of Canadian River Tributary $1 \ldots \ldots \ldots \ldots \ldots$ 10........... 39

Tributary 4 of Canadian River Tributary $1 \ldots \ldots \ldots \ldots \ldots$........ 39

Canadian River Tributary $2 \ldots \ldots \ldots \ldots \ldots \ldots \ldots \ldots \ldots \ldots \ldots \ldots \ldots \ldots \ldots$ 11, 39, 40

Cherry $C$

Chisholm Creek................................. 12,62, 63

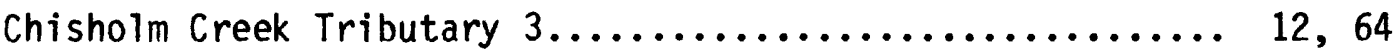

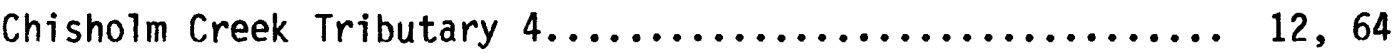

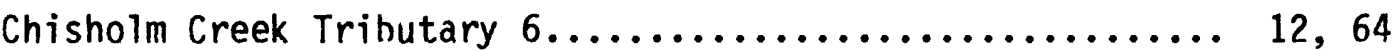

Chisholm Creek Tributary $8 \ldots \ldots \ldots \ldots \ldots \ldots \ldots \ldots \ldots \ldots \ldots \ldots$ 12, 64

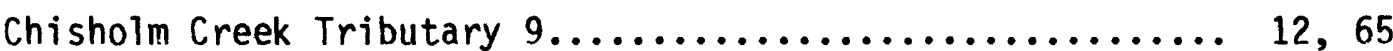

Cloverleaf Creek................................. 12, 61 
Cow Creek........................................... 11, 40

Cow Creek Tributary $1, \ldots \ldots \ldots \ldots \ldots \ldots \ldots \ldots \ldots \ldots \ldots \ldots, 11,41$

Cow Creek Tributary $2 \ldots \ldots \ldots \ldots \ldots \ldots \ldots \ldots \ldots \ldots \ldots \ldots, 11,41,42$

North Branch of Cow Creek Tributary 2............... 11, 42

West Branch of Cow Creek Tributary 2................. 11, 42

Cow Creek Tributary $3 \ldots \ldots \ldots \ldots \ldots \ldots \ldots \ldots \ldots \ldots \ldots \ldots, 11,43$

Crooked Oak Creek...................................... 9, 20-22

Crooked 0ak Creek Tributary A...................... 9, 22, 23

Crutcho Creek........................................ 9, 17, 18

Crutcho Creek Tributary C.......................... 9, 19

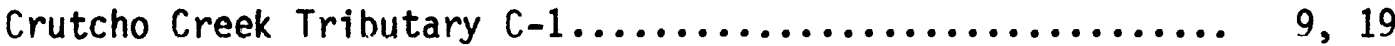

Crutcho Creek Tributary E........................... 9, 18, 19

Crutcho Creek Tributary F....................... 9, 19

Crutcho Creek Tributary G.......................... 9, 20

Deep Fork $. \ldots \ldots \ldots \ldots \ldots \ldots \ldots \ldots \ldots \ldots \ldots \ldots \ldots \ldots \ldots \ldots, 11,44,48$

Deep Fork Tributary $4 . \ldots \ldots \ldots \ldots \ldots \ldots \ldots \ldots \ldots \ldots \ldots, 11,49$

Deep Fork Tributary $5 \ldots \ldots \ldots \ldots \ldots \ldots \ldots \ldots \ldots \ldots \ldots \ldots, 11,49$

Deep Fork Tributary $6 \ldots \ldots \ldots \ldots \ldots \ldots \ldots \ldots \ldots \ldots \ldots \ldots .11,49,50$

Deep Fork Tributary $7 \ldots \ldots \ldots \ldots \ldots \ldots \ldots \ldots \ldots \ldots \ldots \ldots \ldots \ldots \ldots \ldots, 50$

Deep Fork Tributary $9 . \ldots \ldots \ldots \ldots \ldots \ldots \ldots \ldots \ldots \ldots \ldots, 11,53$

Deep Fork Tributary $10 \ldots \ldots \ldots \ldots \ldots \ldots \ldots \ldots \ldots \ldots \ldots . \ldots \ldots \ldots, 54$

Deep Fork Tributary $11 \ldots \ldots \ldots \ldots \ldots \ldots \ldots \ldots \ldots \ldots \ldots, 11,54$

Deep Fork Tributary $12 \ldots \ldots \ldots \ldots \ldots \ldots \ldots \ldots \ldots \ldots \ldots . \ldots \ldots$ 12, 55

Deep Fork Tributary $13 \ldots \ldots \ldots \ldots \ldots \ldots \ldots \ldots \ldots \ldots \ldots, 12,55$

Deep Fork Tributary 13 A.......................... 12, 56

Deep Fork Tributary 13B........................ 12, 56 
Deep Fork Tributary $16 \ldots \ldots \ldots \ldots \ldots \ldots \ldots \ldots \ldots \ldots \ldots \ldots \ldots \ldots \ldots \ldots \ldots \ldots, 56,57$

Deep Fork Tributary 16 West Branch................. 12, 57, 58

Deep Fork Tributary $17 \ldots \ldots \ldots \ldots \ldots \ldots \ldots \ldots \ldots \ldots \ldots \ldots \ldots \ldots \ldots \ldots$

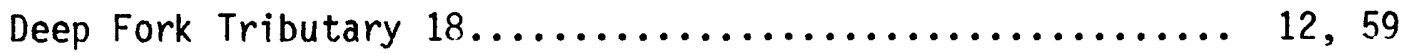

Deep Fork Tributary 18 west Branch.................. 12, 59, 60

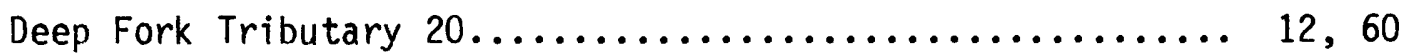

Deep Fork Tributary $22 \ldots \ldots \ldots \ldots \ldots \ldots \ldots \ldots \ldots \ldots \ldots . \ldots \ldots \ldots$

Deer Creek...................................... 13, 65, 66

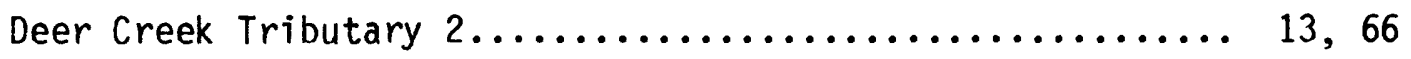

Deer Creek Tributary $3 \ldots \ldots \ldots \ldots \ldots \ldots \ldots \ldots \ldots \ldots \ldots \ldots \ldots \ldots \ldots, 13,68$

Deer Creek Tributary 3 West Branch................. 13, 69

Deer Creek Tributary $4 \ldots \ldots \ldots \ldots \ldots \ldots \ldots \ldots \ldots \ldots \ldots \ldots$ 13, 69

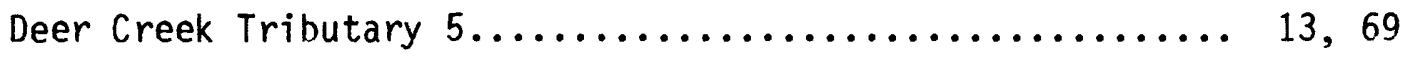

Deer Creek Tributary $6 \ldots \ldots \ldots \ldots \ldots \ldots \ldots \ldots \ldots \ldots \ldots \ldots 13,69,70$

Deer Creek Tributary $7 \ldots \ldots \ldots \ldots \ldots \ldots \ldots \ldots \ldots \ldots \ldots \ldots \ldots \ldots \ldots \ldots \ldots \ldots, 13,70$

Deer Creek Tributary $8 \ldots \ldots \ldots \ldots \ldots \ldots \ldots \ldots \ldots \ldots \ldots \ldots, 13,71$

Deer Creek Tributary $11 \ldots \ldots \ldots \ldots \ldots \ldots \ldots \ldots \ldots \ldots \ldots .13,72$

Deer Creek Tributary $12 \ldots \ldots \ldots \ldots \ldots \ldots \ldots \ldots \ldots \ldots \ldots, 13,72$

Deer Creek Tributary $13 \ldots \ldots \ldots \ldots \ldots \ldots \ldots \ldots \ldots \ldots \ldots \ldots \ldots \ldots \ldots \ldots, 13,72$

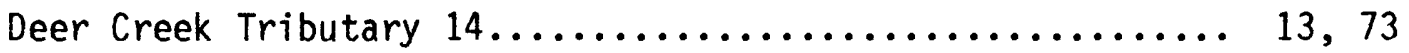

Dry Creek of Bluff Creek........................... 14, 76, 77

Golf Course Creek.................................. 12, 60, 61

Golf Course Creek West Branch.................... 12, 61

Harrison Creek..................................... 11, 50-52

Harrison Creek Tributary $1 \ldots \ldots \ldots \ldots \ldots \ldots \ldots \ldots \ldots \ldots \ldots \ldots \ldots \ldots \ldots, 11,52$

Harrison Creek Tributary $2 \ldots \ldots \ldots \ldots \ldots \ldots \ldots \ldots \ldots \ldots .11,52,53$ 
West Branch Harrison Creek Tributary $2 \ldots \ldots \ldots \ldots \ldots \ldots \ldots 1,53$

Harrison Creek Tributary $3 . \ldots \ldots \ldots \ldots \ldots \ldots \ldots \ldots \ldots \ldots, 11,53$

Hog Creek

$14,83-85$

Hog Creek Tributary $1 \ldots \ldots \ldots \ldots \ldots \ldots \ldots \ldots \ldots \ldots \ldots \ldots, 14,85$

Hog Creek Tributary $2 \ldots \ldots \ldots \ldots \ldots \ldots \ldots \ldots \ldots \ldots \ldots \ldots, 14,85$

Hog Creek Tributary $3 . \ldots \ldots \ldots \ldots \ldots \ldots \ldots \ldots \ldots \ldots \ldots \ldots 14,88$

Hog Creek Tributary $5 \ldots \ldots \ldots \ldots \ldots \ldots \ldots \ldots \ldots \ldots \ldots, 14,88$

Hog Creek Tributary $6 \ldots \ldots \ldots \ldots \ldots \ldots \ldots \ldots \ldots \ldots \ldots \ldots, 14,88$

Hog Creek East Branch......................... 14, 85, 86

Hog Creek West Branch......................... 14, 86, 87

Hog Creek West Branch Tributary $1 \ldots \ldots \ldots \ldots \ldots \ldots \ldots \ldots, 14,87$

Hog Creek West Branch Tributary $2 \ldots \ldots \ldots \ldots \ldots \ldots \ldots \ldots, 14,87$

Hog Creek West Bracnh Tributary $3 \ldots \ldots \ldots \ldots \ldots \ldots \ldots \ldots, 14,87$

Lightning Creek................................ 9, 23, 24

Lightning Creek Tributary $1 \ldots \ldots \ldots \ldots \ldots \ldots \ldots \ldots \ldots \ldots ., 9,24,25$

Lightning Creek Tributary $3 \ldots \ldots \ldots \ldots \ldots \ldots \ldots \ldots \ldots \ldots$ 9, 25

Lightning Creek Tributary $6 \ldots \ldots \ldots \ldots \ldots \ldots \ldots \ldots \ldots \ldots, 9,25$

Mustang Creek.................................... 10, 30

Mustang Creek Tributary $1 \ldots \ldots \ldots \ldots \ldots \ldots \ldots \ldots \ldots \ldots \ldots, 10,31$

Mustang Creek Tributary 1 west Branch................ 10, 31

Mustang Creek Tributary $2 \ldots \ldots \ldots \ldots \ldots \ldots \ldots \ldots \ldots \ldots \ldots, 10,31$

Mustang Creek Tributary 2 South Branch............... 10, 32

Mustang Creek Tributary $3 \ldots \ldots \ldots \ldots \ldots \ldots \ldots \ldots \ldots \ldots .10,32$

Mustang Creek Tributary 3 East Branch................ 10, 32

Mustang Creek Tributary 3 West Branch................ 10, 33

Mustang Creek Tributary $4 \ldots \ldots \ldots \ldots \ldots \ldots \ldots \ldots \ldots \ldots, 10,33$ 
North Canadian River Tributary $8, \ldots \ldots \ldots \ldots \ldots \ldots \ldots \ldots \ldots \ldots, 9,16,17$

North Canadian River Tributary $9 . \ldots \ldots \ldots \ldots \ldots \ldots \ldots \ldots \ldots \ldots ., 9,17$

North Canadian River Tributary $10 \ldots \ldots \ldots \ldots \ldots \ldots \ldots \ldots \ldots . . . . \ldots, 27$

North Canadian River Tributary $12 \ldots \ldots \ldots \ldots \ldots \ldots \ldots \ldots \ldots \ldots, 9,27$

North Canadian River Tributary $13 \ldots \ldots \ldots \ldots \ldots \ldots \ldots \ldots \ldots, 9,28$

North Canadian River Tributary 14.................... 10, 29, 30

Shell creek....................................... 10, 33, 34

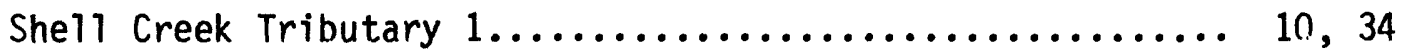

Shell Creek Tributary $2 \ldots \ldots \ldots \ldots \ldots \ldots \ldots \ldots \ldots \ldots \ldots, 10,35$

Shel1 Creek Tributary $3 \ldots \ldots \ldots \ldots \ldots \ldots \ldots \ldots \ldots \ldots \ldots, 10,35$

Shell Creek Tributary $4 \ldots \ldots \ldots \ldots \ldots \ldots \ldots \ldots \ldots \ldots \ldots .10,35,36$

Shell Creek Tributary 4 West Branch................. 1n, 36

Shel1 Creek Tributary 5 ........................ 10, 36, 37

Shell Creek Tributary 5 East Branch.................. 10, 37

Shell Creek Tributary $6 \ldots \ldots \ldots \ldots \ldots \ldots \ldots \ldots \ldots \ldots \ldots, 10,37$

Silver Creek of Spring Creek........................... 14, 82

Spring Creek of Bluff Creek............................. 14, 78-80

Spring Creek West Branch........................ 14, 80,81

Spring Creek of Deer Creek............................ 13, 70, 71

Twin Creek......................................... 9, 25, 26

Warnut Creek....................................... 13, 66-68 